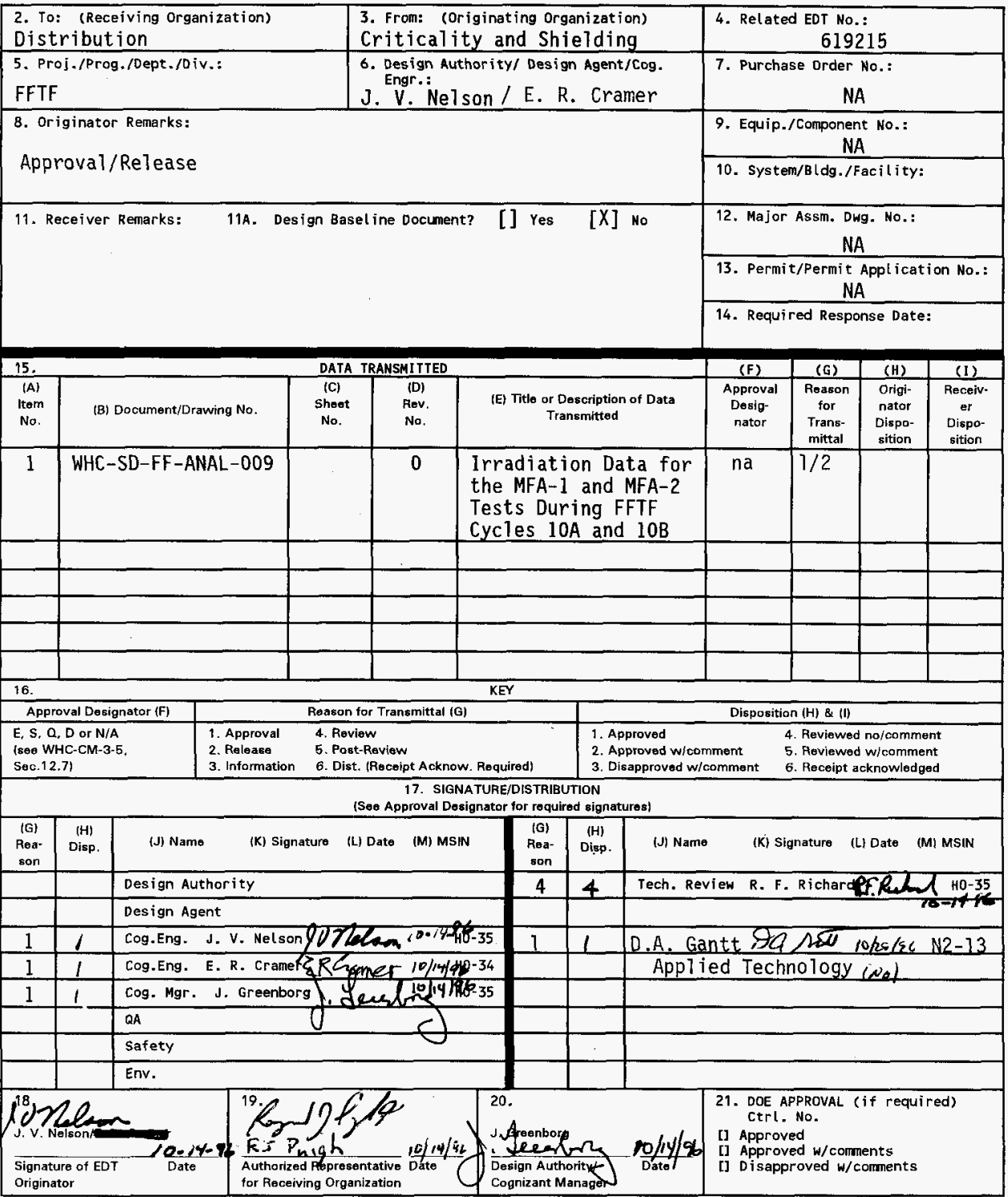

BD-7400-172-2 (05/96) GEF097 


\section{Irradiation Data for the MFA-1 and MFA-2 Tests During FFTF Cycles 10A and 10B}

J. V. Nelson and E. R. Cramer

Westinghouse Hanford Company, Richland, WA 99352

U.S. Department of Energy Contract DE-AC06-87RL10930

$\begin{array}{llll}\text { EDT/ECN: } & 619215 & \text { UC: } 532 \\ \text { Org Code: } & 403 \text { 8M730 } & \text { Charge Code: B193D } \\ \text { B\&R Code: } & \text { 1819930301 } & \text { Total Pages: } 143\end{array}$

Key Words: FFTF, MONJU, Liquid Metal Reactor, fuel test, LMR, MFA

Abstract: This report provides key information on the irradiation environment of the MONJU fuel tests MFA-land MFA-2 in the Fast Flux Tests Facility (FFTF) during operating cycles $10 \mathrm{~A}$ and $10 \mathrm{~B}$. This information includes the fission powers, neutron fluxes, sodium temperatures and sodium flow rates in MFA-1, MFA-2 and adjacent assemblies. It also includes MFA-1 and MFA-2 compositions as a function of exposure during cycles $10 \mathrm{~A}$ and $10 \mathrm{~B}$. The work was performed at the request of Power Reactor and Nuclear Fuels Corporation (PNC) of Japan.

TRADEMARK DISCLAIMER. Reference herein to any specific comercial product, process, or service by trade name, trademark, manufacturer, or otherwise, does not necessarily constitute or imply its endorsement, recommendation, or favoring by the United States Government or any agency thereof or its contractors or subcontractors.

Printed in the United states of America. To obtain copies of this document, contact: WHC/BCS Document Control Services, P.O. Box 1970, Mailstop H6-08, Richland WA 99352, Phone (509) 372-2420. Fax (509) $376-4989$.
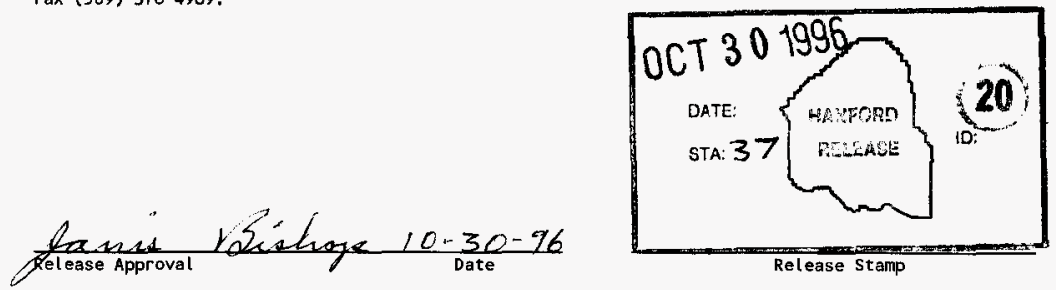

\section{Approved for Public Release}




\section{IRRADIATION DATA FOR THE MFA-1 AND MFA 2 TESTS DURING FFTF CYCLES 1OA AND 1OB}

This report provides data relevant to the irradiation of the MONJU fuel experiments MFA-1 and MFA-2 in the Fast Flux Test Facility (FFTF) during operating cycles $10 A-1,10 A-2,10 A-3,10 A-4$ and $10 B$. The operating history of these cycles is summarized in Table 1 . The data are supplied in both tabular and electronic forms, as available.

Data provided for MFA-1 and MFA-2 include:

- assembly fission powers, total neutron fluxes, outlet temperatures and coolant flow rates,

spatial distributions of total flux, fission power and fast flux (flux greater than $0.1 \mathrm{MeV}$ at the duct walls),

subchannel sodium temperatures, and

burnup dependant material compositions.

Also included are powers, outlet temperatures, and flow rates for the assemblies adjacent to MFA-1 and MFA-2. In general, these data are provided for the beginning and end of each of the listed operating cycles.

MFA- 1 was irradiated in FFTF core lattice position 1506 during cycles $10 \mathrm{~A}$ and 10B, while MFA-2 was irradiated in position 2507 , as shown in Figure 1 . This figure shows the FFTF core loading for cycle 10A-1. Loading changes made in core locations adjacent to MFA-1 and MFA-2 between cycles $10 \mathrm{~A}-1$ and $10 \mathrm{~B}$ are identified in Table 2 .

Enclosed are 18 different tables for each cycle. Some of these tables were generated for beginning-of-cycle (BOC) conditions, some were generated for end-of-cycle (EOC) conditions, and many were generated for both BOC and EOC conditions. The reasons for this are explained later in sections describing the different tables. Most tables are also provided in electronic form on disk. However, others were available only in hard copy form from previous computer runs. These runs could not be repeated to obtain disk files because the computer programs needed have not been installed on any computer system that is currently available.

Table 3 summarizes the information contained in each of the 18 data tables, indicates whether this information is provided for $B O C$, EOC or both, and identifies the tables that are also on the enclosed disk. The enclosed data (both printed tables and electronic files) are identified using the following notation: TCCC.N, where $T$ is either the letter B (for beginning of cycle) or $E$ (for end of cycle), $C C C$ is a cycle name (i.e., 10A-1, 10A-2,.., 10B), and $N$ is a table number $(1-17)$ listed in Table 3. For example, Table B10A-4.1 lists the fission power generated in MFA-1, MFA-2 and neighboring assemblies at the beginning of cycle 10A-4 (BOC 10A-4).

The following paragraphs explain the contents of each of the 18 types of tables. 


\section{Assembly Fission Power}

The fission power generated in MFA-1 and each of its six adjacent assemblies, and in MFA-2 and each of its six adjacent assemblies are 1 isted in Tables $B 10 A-1.1, E 10 A-1.1, B 10 A-2.1, E 10 A-2.1$, etc. In these tables, the total fission power is given for each assembly. Also, a breakdown of the power by axial material region (fuel, axial blankets and $\mathrm{UO}_{2}$ insulator pellets) is included. Fuel assemblies that do not have axial blankets have insulator pellets between the fuel and the axial reflector regions.

Fission powers were computed using the three-dimensional diffusion theory and burnup program 3DB with nuclear cross section data in 12 energy groups. The computed flux and power data were adjusted by applying row-dependant bias factor that ranged in value from 0.98 to 1.02 . For row 5 where MFA-1 and MFA-2 are located, the bias factor applied was 0.984 . The bias factors were determined by comparing the results of measurements made as part of the FFTF reactor characterization program, diffusion theory calculations and Monte Carlo (MCNP) calculations.

\section{Assembly Averaged Total Flux}

Assembly averaged total flux data for MFA-1 and MFA-2 at the beginning and end of each cycle are given in Tables B10A-1.2, E10A-1.2, B10A-2.2, E10A-2.2, etc. In computing the data in these tables, only the fuel region of each assembly was included in the averaging process. The flux data averaged were obtained from the same 3DB calculations used to produce the fission power data. Again, the bias factor of 0.984 was applied to the 3DB results.

\section{Axial Distribution of Total Flux, Fast Flux and Power}

The axial distributions of total flux, fast flux (flux greater than $0.1 \mathrm{MeV}$ ) and fission power are given for MFA-1 at the beginning and end of each cycle in Tables BlOA-1.3, E10A-1.3, BlOA-2.3, El0A-2.3, etc. Likewise, the axial distributions of total flux, fast flux and fission power are given for MFA-2 in Tables BlOA-1.4, E1OA-1.4, B10A-2.4, E10A-2.4, etc.

The axial locations relative to the core midplane in units of centimeters $(\mathrm{cm})$ at hot, full-power conditions are given in the first column of each table. Negative values indicate locations below the midplane, and positive values indicate locations above the midplane. The flux and power data in the tables are normalized so that the axial average of each distribution over the active core region is 1.0 . The active core region extended from $-46.14 \mathrm{~cm}$ to 46.14 $\mathrm{cm}$ in the 3DB models of hot, full-power conditions in FFTF.

\section{Fission Power Distribution by Pin}

The distribution of fission power by pin is given for MFA-1 at the beginning and end of each cycle in Tables B10A-1.5, E10A-1.5, B10A-2.5, E10A-2.5, etc. Likewise, the distribution of fission power by pin is given for MFA-2 in Tables BIOA-1.6, ElOA-1.6, B1OA-2.6, ElOA-2.6, etc. The direction north (N) is indicated in these tables.

The data in each table were based on a power distribution computed by 3DB. These distributions had six data points per assembly at each axial elevation. Pin power data were generated by first axially averaging the 3DB computed 
powers, and then doing a least-squares quadratic fit to the available data. Finally, the pin powers obtained were normalized so that the linear power averaged over the fuel region of an assembly is 1.0 .

Using the given assembly powers, the axial power distributions and the pin power distributions, the linear power in any MFA-1 or MFA-2 pin at any axial elevation can be easily determined.

\section{Total Flux Distribution by Pin}

The distribution of total flux by pin in MFA-1 is given for the beginning and end of each cycle in Tables BIOA-1.7, E1OA-1.7, BIOA-2.7, E1OA-2.7, etc. Likewise, the distribution of total flux by pin in MFA-2 is given in Tables $B 10 A-1.8, E 10 A-1.8, B 10 A-2.8, E 10 A-2.8$, etc. These data were generated using the same method described above that was used to obtain the pin powers. Again, the flux data were normalized so that the average total flux in the fuel region of MFA-1 or MFA-2 is 1.0 .

\section{Duct Wall Flux Greater than $0.1 \mathrm{MeV}$}

The fluxes greater than $0.1 \mathrm{MeV}$ at each of the six faces of the MFA-1 and MFA-2 ducts are given in Tables B1OA-1.9, E1OA-1.9, BIOA-2.9, E1OA-2.9, etc. The data given represent axial averages. To obtain the duct wall flux at a particular axial elevation, multiply by an axial factor from Tables B10A-1.3, B10A-1.4, E10A-1.3, E10A-1.4, etc.

Each of the six duct walls in each assembly is identified in the tables by the direction it faces - east (E), southeast (SE), southwest (SW), west (W), northwest (NW), or northeast (NE). Figure 1 shows the orientation of MFA-1 in lattice position 1506 and MFA-2 in lattice position 2507. For example, the east (E) duct wall of MFA-1 is adjacent to the assembly in lattice position 1505, and the east duct wall of MFA-2 is adjacent to the assembly in lattice position 2508 .

The data in these tables were based on group flux distributions computed by $3 D B$. The duct wall fluxes were generated by axially averaging the $30 \mathrm{~B}$ fluxes, integrating over energy to obtain the flux greater than $0.1 \mathrm{MeV}$ at each space point, doing a linear interpolation between the two flux values on each side of a duct wall face, and finally applying the 0.984 adjustment factor described earlier.

\section{Sodium Outlet Temperatures and Assembly Flow Rates}

Sodium outlet temperatures and flow rates for MFA-1, MFA-2 and the assemblies adjacent to them are given in Tables B10A-1.10, E10A-1.10, B10A-2.10, etc. Computed and measured temperatures are included in these tables as available from FFTF core reload design reports and cycle reports. Both measured and computed temperatures are given for $B O C 10 A-1,10 A-2,10 A-4$ and $10 B$, and for EOC $10 \mathrm{~A}-4$ and $10 \mathrm{~B}$. Computed temperatures only are given for EOC 10A-1 and $10 \mathrm{~A}-2$. Measured temperatures only are given for $\mathrm{BOC} 10 \mathrm{~A}-3$ and $10 \mathrm{~A}-4$. No temperature or flow data are given for EOC 1OA-3. Because of the short length of cycle 10A-3 (5.8 EFPO), the EOC 10A-3 data will be nearly the same as the $80 C$ 10A-3 data. 
A11 assembly flow rate data were calculated for full power conditions. For cycle 10A-1, assembly flows were based on a calculated pressure drop of 113.3 psi at the total reactor flow rate of $16.74 \times 10^{6} \mathrm{lb} / \mathrm{hr}$. For cycies $10 \mathrm{~A}-2$, 10A-3 and 10A-4, assembly flows were based on a calculated pressure drop of 110.6 psi. For cycle $10 \mathrm{~B}$, assembly flows were based on a calculated pressure drop of 111.5 psi.

\section{Sodium Subchannel Temperatures}

Calculated sodium subchannel temperatures at three axial 1ocations are available from previous analyses for the following times: $B O C$ 10A-1, $B O C$ $10 \mathrm{~A}-2$, EOC $10 \mathrm{~A}-2$, $B O C \mathrm{C} 10 \mathrm{~B}$ and $\mathrm{EOC} 10 \mathrm{~B}$. No data are available for cycles $10 \mathrm{~A}-3$ and 1OA-4. However, sodium subchannel temperatures given for EOC 1OA-2 should be very close to the temperatures during cycles 10A-3 and 10A-4 because of the short length of these cycles and the very minor loading differences.

Subchannel temperatures at the top of core elevation are given for MFA- 1 in Tables B1OA-1.11, B1OA-2.11, E1OA-2.11, B1OB.11 and E1OB.11, and for MFA-2 in Tables $B 10 A-1.12, B 10 A-2.12$, etc. Subchannel temperatures at the top of the upper axial blanket are given for MFA-1 in Tables B10A-1.13, B10A-2.13, etc, and for MFA-2 in Tables BlOA-1.14, B10A-2.14, etc. Subchannel temperatures at top of the fuel pin bundle are given for MFA-1 in Tables B10A-1.15, B10A-2.15, etc, and for MFA-2 in Tables BIOA-1.16, B10A-2.16, etc.

All temperatures are in degrees Fahrenheit. The row of numbers just outside the asterisks on each map are calculated duct wall temperatures, and the outside row of temperatures on each map are the temperatures of the sodium between assemblies. The direction north is indicated on each map.

The temperature calculations were performed using the SUPERENERGY code. The power of each pin, the assembly flow distribution and the reactor inlet temperature of $680^{\circ} \mathrm{F}$ were input to the code. SUPERENERGY calculates subchannel temperatures by using energy balances and correlations for subchannel mixing. SUPERENERGY has not been installed on any computer system that is currently available. For this reason, the calculations could not be repeated to obtain electronic copies of the temperature data, and data for cycles $10 \mathrm{~A}-3$ and $10 \mathrm{~A}-4$.

Fuel pin cladding temperatures are not available, but can be computed from the data provided.

\section{Material Compositions}

Burnup-dependant material compositions for MFA-1 at the end of each cycle are given in Tables ElOA-1.17, ElOA-2.17, etc, while the compositions for MFA-2 are given in Tables E1OA-1.18, E18A2.18, etc. Each table 1ists homogenized material compositions for 11 axial regions in MFA-1 or MFA-2. These 11 regions are identified in Table 4 . The axial extent of each region, as modeled in $3 \mathrm{DB}$, is listed in the material composition tables. The units are centimeters $(\mathrm{cm})$ relative to the core midplane at hot, full power conditions. The compositions are in units of atoms/b-cm at hot, full power conditions. Both the fresh (beginning-of-life) and end-of-cycle compositions are included in each table. Compositions of MFA-1 and MFA-2 at BOC $10 A-1$ were the fresh values listed. Compositions at the beginning of any other cycle were 
essentially those listed for the end of the previous cycle. The only differences are due to decay of ${ }^{241} \mathrm{Pu}$ to ${ }^{241} \mathrm{Am}$.

The burnup-dependant compositions for the axial blankets (axial regions 3 and 10) and the fuel (axial regions 4 through 9) were computed using the threedimensional diffusion theory and burnup code, 3DB. The fuel was subdivided into six axial regions to properly account for the effect that the axial flux distribution has on the fuel depletion rate.

The constituents of the materials listed in the composition tables are individual isotopes with two exceptions. The first exception is that the constituent labelled "FP" represents an average pseudo fission product pair. An atom density for FP specifies the concentration of fission product pairs, not individual fission product nuclides. The microscopic cross section data used for fission products in the 3DB calculations were generated assuming all fissions were from ${ }^{239} \mathrm{Pu}$.

The second exception is that the constituent labelled "SS-316" represents type 316 stainless steel. An atom density given for \$S-316 indicates the total number of atoms in stainless steel per unit volume. SS-316 was used in the calculational models for the ducts, cladding and wire wrap in both MFA-1 and MFA-2, even though these components in MFA-2 were fabricated from an advanced austenitic stainless steel. The composition used in generating nuclear cross section sets for SS-316 was based on the 1977 ROT \& ASTM standard, and is listed in Table 5 .

\section{Contents of the Enclosed Disk}

The enclosed 3.5 inch floppy disk is DOS formatted with 1.44 MB capacity. All information on the disk is contained in standard text (ASCII) files. There are five directories on the disk, one for each cycle. The directory names are $10 \mathrm{~A}-1,10 \mathrm{~A}-2,10 \mathrm{~A}-3,10 \mathrm{~A}-4$ and 10-B. As described earlier, the files on the disk are named the same as the data tables. For example, assembly fission powers for EOC 10A-4 are listed in Table ElOA-4.1 and on disk file E10A-4.1, which is located in directory 10A-4. Table 3 indicates which of the data tables for each cycle are included on the disk. Also, a table of contents of the disk is given in Table 6 .

Table 1. Summary of FFTF Cycles 10A and 10B Power Operation

\begin{tabular}{||c|c|c|c||}
\hline Cycle & $\begin{array}{c}\text { Startup } \\
\text { Date }\end{array}$ & $\begin{array}{c}\text { Shutdown } \\
\text { Date }\end{array}$ & $\begin{array}{c}\text { Cycle } \\
\text { Length } \\
\text { (EFPD }^{2} \text { ) }\end{array}$ \\
\hline $10 \mathrm{~A}-1$ & $11 / 18 / 87$ & $1 / 26 / 88$ & 66.3 \\
\hline $10 \mathrm{~A}-2$ & $2 / 1 / 88$ & $2 / 23 / 88$ & 20.3 \\
\hline $10 \mathrm{~A}-3$ & $2 / 25 / 88$ & $3 / 4 / 88$ & 5.8 \\
\hline $10 \mathrm{~A}-4$ & $3 / 7 / 88$ & $5 / 7 / 88$ & 59.4 \\
\hline $10 \mathrm{~B}$ & $6 / 11 / 88$ & $10 / 17 / 88$ & 126.7 \\
\hline
\end{tabular}

${ }^{a}$ EFPD = equivalent full power days. Full power was 291 MW thermal. 


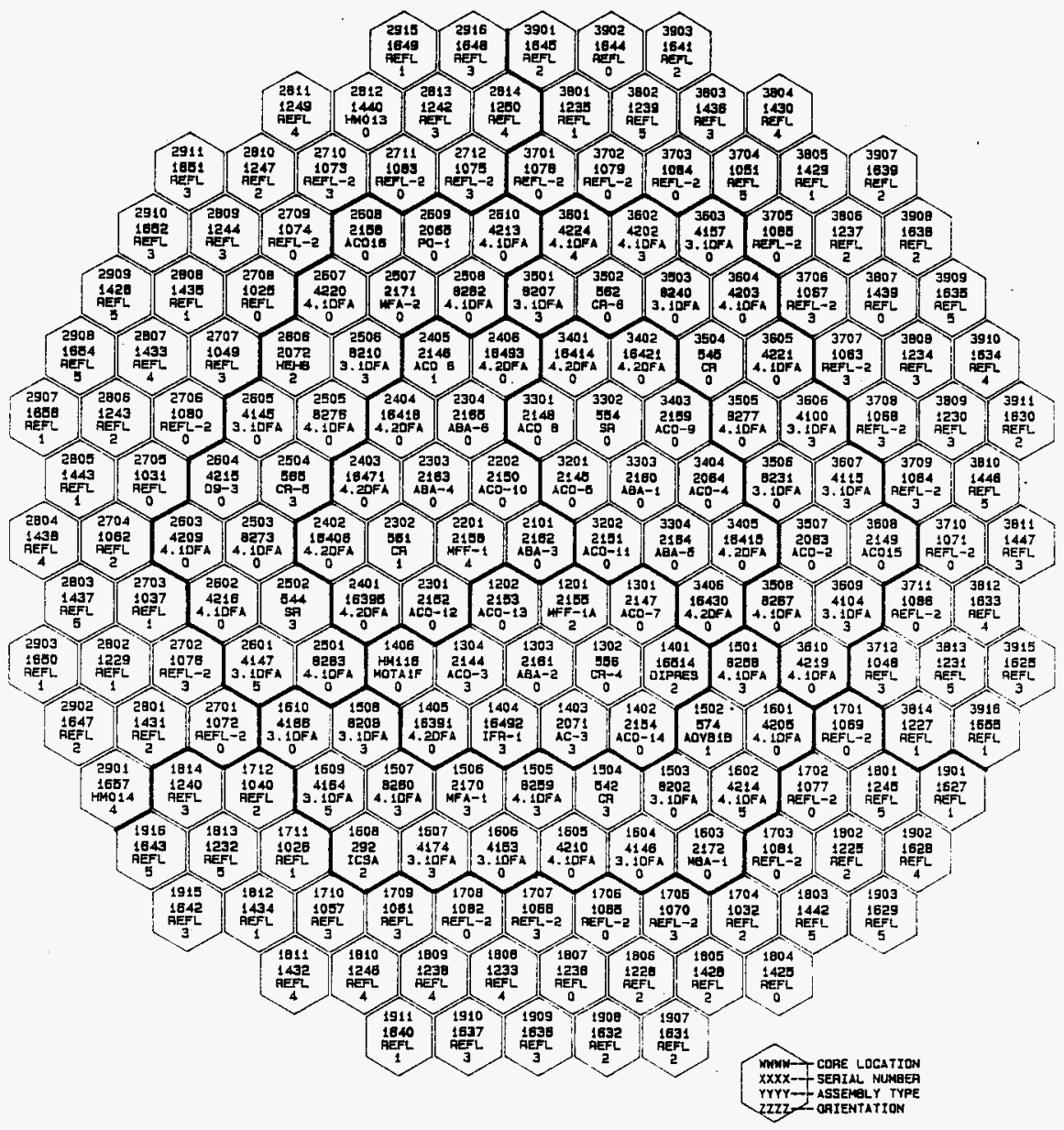

Figure 1. FFTF Cycle 10A-1 Core Loading 
Table 2. Loading Changes Made to Core Locations adjacent to MFA-1 and MFA-2

\begin{tabular}{|c|c|c|c|c|c|}
\hline \multirow{2}{*}{$\frac{\text { Cycle }}{10 A-2}$} & \multirow{2}{*}{$\begin{array}{c}\begin{array}{c}\text { Core } \\
\text { Position }\end{array} \\
2608\end{array}$} & \multicolumn{2}{|c|}{$\begin{array}{l}\text { Assembly Removed } \\
\text { (Serial No. \& Type) }\end{array}$} & \multicolumn{2}{|c|}{$\begin{array}{l}\text { Assembly Inserted } \\
\text { (serial No. \& Type) }\end{array}$} \\
\hline & & 2156 & $A C 0-16^{C}$ & 4114 & 3.1DFA \\
\hline $10 A-4$ & 2609 & 2065 & $P O-1^{d}$ & 4140 & 3.1DFA \\
\hline \multirow[t]{5}{*}{$10 \mathrm{~B}$} & 1405 & 16391 & 4.20FA & 2168 & $M F F-2^{e}$ \\
\hline & 1505 & 8259 & 4.1DFA & 8265 & 4.1DFA \\
\hline & 2405 & 2146 & $A C 0-6^{C}$ & 16396 & 3. $2 \mathrm{DFA}^{\mathrm{a}}$ \\
\hline & 2506 & 8210 & 3.1DFA & 8268 & 4.1DFA \\
\hline & 2609 & 4140 & 3.1DFA & 4152 & 4.1DFA \\
\hline
\end{tabular}

3.1DFA and 3.2DFA are standard FFTF series I drivers

b 4.1DFA and 4.20FA are standard FFTF series II drivers

c ACO- 6 and ACO-16 are Core Demonstration Experiment (CDE) fuel assemblies

d $P O-1$ is an advanced oxide fuel test

e MFF-2 is a metal fuel experiment 
Table 3. Summary Data Included for each Cycle

\begin{tabular}{|c|c|c|c|}
\hline $\begin{array}{l}\text { Table } \\
\text { No. }\end{array}$ & Description & $\begin{array}{l}\text { Created for } \\
\text { BOC or EOC? }\end{array}$ & $\begin{array}{l}\text { Included } \\
\text { on disk? }\end{array}$ \\
\hline 1 & Fission Power Generated in MFA-1, MFA-2 and Neighboring Assembl ies & BOC \& EOC & yes \\
\hline 2 & Assembly Averaged Total Flux for MFA-1 and MFA-2 & $B O C \& E O C$ & yes \\
\hline 3 & Axial Distribution of Total Flux, Fast Flux and Power in MFA- 1 & $B O C \& E O C$ & yes \\
\hline 4 & Axial Distribution of rotal Flux, Fast Flux and Power in MFA-2 & BOC \& EOC & yes \\
\hline 5 & Fission Power Distribution by Pin in MFA-1 & BOC \& EOC & yes \\
\hline 6 & Fission Power Distribution by Pin in MFA-2 & $B O C \& E O C$ & yes \\
\hline 7 & Total Flux Distribution by $P$ in in MFA-1 & $B O C \& E O C$ & yes \\
\hline 8 & Totat Flux Distribution by Pin in MFA-2 & $B O C \& E O C$ & yes \\
\hline 9 & MFA-1 and MFA-2 Duct Wall Flux Greater than $0.1 \mathrm{MeV}$ & BOC \& EOC & yes \\
\hline 10 & $\begin{array}{l}\text { Sodium Outlet Temperatures and Flow Rates for MFA-1, MFA-2 and } \\
\text { Neighboring Assemblies }\end{array}$ & BOC \& EOC ${ }^{a}$ & yes \\
\hline 11 & MFA- 1 Sodium Subchannel Temperatures at Top of Core Elevation & $B O C \& \operatorname{EOC}^{b}$ & no \\
\hline 12 & MFA-2 Sodium Subchannel Temperatures at Top of Core Elevation & $B O C \& E^{b} C^{b}$ & no \\
\hline 13 & $\begin{array}{l}\text { MFA-1 Sodium Subchannel Temperatures at Elevation of Upper Axial } \\
\text { Blanket }\end{array}$ & $B O C \& E^{b} C^{b}$ & no \\
\hline 14 & $\begin{array}{l}\text { MFA-2 Sodium Subchannel Temperatures at Elevation of Upper Axial } \\
\text { Blanket }\end{array}$ & $B O C \& E^{b} C^{b}$ & no \\
\hline 15 & MFA-1 sodium subchannel Temperatures at Top of Fuel Pin Bundte & $\mathrm{BOC} \& \mathrm{EOC}^{\mathrm{b}}$ & no \\
\hline 16 & MFA-2 Sodium Subchannel Temperatures at Top of Fuel Pin Bundle & $B O C \& \operatorname{EOC}^{b}$ & no \\
\hline 17 & Composition of MFA-1 & EOC & yes \\
\hline 18 & Composition of MFA- 2 & EOC & yes \\
\hline
\end{tabular}

a No table of sodium outlet temperatures and flow rates for EOC $10 \mathrm{~A}-3$ is included because of the short cycle length (5.8 EFPD). Temperatures and flow rates at EOC 10A-3 are essentially the same as given for BOC $10 \mathrm{~A}-3$.

b Sodium subchannel temperatures are available for only BOC 10A-1, 10A-2 and 10B, and EOC 10A-2 and 10B. 
Table 4. Description of Axial Regions in Material Composition Tables

\begin{tabular}{||c|c|c|}
\hline $\begin{array}{c}\text { Axial } \\
\text { Level }\end{array}$ & $\begin{array}{c}\text { Axial Range } \\
\text { Relative to Core } \\
\text { Midplane }(\mathrm{cm})^{\mathrm{a}}\end{array}$ & \multicolumn{1}{c|}{ Description } \\
\hline 1 & $-100.28^{\mathrm{b}}$ to -75.28 & Lower axial shield \\
\hline 2 & -75.28 to -62.78 & Pin attachment region \\
\hline 3 & -62.78 to -46.14 & Lower axial blanket \\
\hline $4-9$ & $\begin{array}{c}-46.14 \text { to } 46.14 \\
\text { (divided into } 6 \text { regions, } \\
\text { each } 15.38 \mathrm{~cm} \text { high) }\end{array}$ & Fuel region \\
\hline 10 & 46.14 to $62.78^{\mathrm{c}}$ & Upper axial blanket \\
\hline 11 & 62.78 to $116.00^{\mathrm{b}}$ & Gas plenum \\
\hline
\end{tabular}

a Dimensions are representative of hot, full power conditions. Negative numbers indicate locations below the core midplane. Positive numbers indicate locations above the core midplane.

b The axial range in the $30 B$ models was $100.28 \mathrm{~cm}$ below the core midplane to $116.00 \mathrm{~cm}$ above the midplane.

c The upper axial blankets of MFA-1 and MFA-2 are actually $7.62 \mathrm{~cm}$ (3 in.) long. However, they were modeled as being $16.64 \mathrm{~cm}$ long (at hot, full power conditions) to match existing axial boundaries in the 3DB model.

Table 5. Composition of Type 316 Stainless Steel (SS-316)

\begin{tabular}{|c|c||c|c||}
\hline Constituent & $\begin{array}{c}\text { Weight } \\
\text { Percent }\end{array}$ & Constituent & $\begin{array}{c}\text { Weight } \\
\text { Percent }\end{array}$ \\
\hline $\mathrm{Fe}$ & 62.19 & $\mathrm{Mn}$ & 2.00 \\
\hline $\mathrm{Cr}$ & 18.00 & $\mathrm{Si}$ & 0.75 \\
\hline $\mathrm{Ni}$ & 14.00 & $\mathrm{C}$ & 0.06 \\
\hline $\mathrm{Mo}$ & 3.00 & & \\
\hline
\end{tabular}


Table 6. Disk Table of Contents (Sheet 1 of 2)

\begin{tabular}{|c|c|c|c|c|c|c|c|c|c|}
\hline \multicolumn{2}{|c|}{ File Name } & \multirow{2}{*}{$\begin{array}{c}\text { Size } \\
\text { (bytes) }\end{array}$} & \multicolumn{2}{|c|}{ Creation } & \multicolumn{2}{|c|}{ File Name } & \multirow{2}{*}{$\begin{array}{c}\text { Size } \\
\text { (bytes) }\end{array}$} & \multicolumn{2}{|c|}{ Creation } \\
\hline Base & Ext. & & Date & Time & Base & Ext. & & Date & Time \\
\hline \multicolumn{5}{|c|}{ Directory of $10 \mathrm{~A}-1$} & \multicolumn{5}{|c|}{22 files, 40,092 bytes total } \\
\hline $\begin{array}{l}\text { B1OA-1 } \\
\text { B1OA-1 } \\
\text { B1OA-1 } \\
\text { B1OA-1 } \\
\text { B1OA-1 } \\
\text { B1OA-1 } \\
\text { B1OA-1 } \\
\text { B10A-1 } \\
\text { B10A-1 } \\
\text { B1OA-1 }\end{array}$ & $\begin{array}{l}1 \\
10 \\
2 \\
3 \\
4 \\
5 \\
6 \\
7 \\
8 \\
9\end{array}$ & $\begin{array}{r}984 \\
738 \\
205 \\
2,757 \\
2,748 \\
1,561 \\
1,561 \\
1,558 \\
1,560 \\
349\end{array}$ & $\begin{array}{l}10-02-96 \\
10-02-96 \\
09-27-96 \\
09-27-96 \\
09-27-96 \\
09-27-96 \\
09-27-96 \\
09-27-96 \\
09-27-96 \\
09-27-96\end{array}$ & $\begin{array}{r}3: 19 p \\
3: 21 p \\
11: 12 a \\
11: 13 a \\
11: 13 a \\
11: 13 a \\
11: 13 a \\
11: 13 a \\
11: 13 a \\
11: 13 a\end{array}$ & $\begin{array}{l}\text { E10A-1 } \\
\text { E10A-1 } \\
\text { E10A-1 } \\
\text { E10A-1 } \\
\text { E10A-1 } \\
\text { E10A-1 } \\
\text { E10A-1 } \\
\text { E10A-1 } \\
\text { E10A-1 } \\
\text { E10A-1 } \\
\text { E10A-1 } \\
\text { E1OA-1 }\end{array}$ & $\begin{array}{l}1 \\
10 \\
17 \\
18 \\
2 \\
3 \\
4 \\
5 \\
6 \\
7 \\
8 \\
9 \\
\end{array}$ & $\begin{array}{r}984 \\
583 \\
6,101 \\
6,101 \\
205 \\
2,759 \\
2,748 \\
1,561 \\
1,561 \\
1,558 \\
1,558 \\
352 \\
\end{array}$ & $\begin{array}{l}09-27-96 \\
10-02-96 \\
09-27-96 \\
09-27-96 \\
09-27-96 \\
09-27-96 \\
09-27-96 \\
09-27-96 \\
09-27-96 \\
09-27-96 \\
09-27-96 \\
09-27-96 \\
\end{array}$ & $\begin{array}{r}11: 14 a \\
3: 22 p \\
11: 14 a \\
11: 14 a \\
11: 14 a \\
11: 14 a \\
11: 14 a \\
11: 14 a \\
11: 14 a \\
11: 14 a \\
11: 14 a \\
11: 15 a\end{array}$ \\
\hline \multicolumn{5}{|c|}{ Directory of $10 \mathrm{~A}-2$} & \multicolumn{5}{|c|}{22 files, 40,087 bytes total } \\
\hline $\begin{array}{l}\text { B10A-2 } \\
\text { B10A-2 } \\
\text { B10A-2 } \\
\text { B10A-2 } \\
\text { B10A-2 } \\
\text { B10A-2 } \\
\text { B10A-2 } \\
\text { B10A-2 } \\
\text { B10A-2 } \\
\text { B10A-2 }\end{array}$ & $\begin{array}{l}1 \\
10 \\
2 \\
3 \\
4 \\
5 \\
6 \\
7 \\
8 \\
9\end{array}$ & $\begin{array}{r}984 \\
738 \\
205 \\
2,758 \\
2,748 \\
1,563 \\
1,561 \\
1,558 \\
1,558 \\
349\end{array}$ & $\begin{array}{l}09-27-96 \\
10-02-96 \\
09-27-96 \\
09-27-96 \\
09-27-96 \\
09-27-96 \\
09-27-96 \\
09-27-96 \\
09-27-96 \\
09-27-96\end{array}$ & $\begin{array}{r}11: 27 a \\
3: 32 p \\
11: 27 a \\
11: 27 a \\
11: 27 a \\
11: 27 a \\
11: 27 a \\
11: 27 a \\
11: 27 a \\
11: 27 a\end{array}$ & $\begin{array}{l}\text { E1OA-2 } \\
\text { E1OA-2 } \\
\text { E1OA-2 } \\
\text { E1OA-2 } \\
\text { E1OA-2 } \\
\text { E1OA-2 } \\
\text { E1OA-2 } \\
\text { E10A-2 } \\
\text { E1OA-2 } \\
\text { E1OA-2 } \\
\text { E1OA-2 } \\
\text { E1OA-2 } \\
\end{array}$ & $\begin{array}{l}1 \\
10 \\
17 \\
18 \\
2 \\
3 \\
4 \\
5 \\
6 \\
7 \\
8 \\
9 \\
\end{array}$ & $\begin{array}{r}984 \\
582 \\
6,101 \\
6,101 \\
205 \\
2,752 \\
2,749 \\
1,561 \\
1,561 \\
1,558 \\
1,558 \\
353 \\
\end{array}$ & $\begin{array}{l}09-27-96 \\
09-27-96 \\
09-27-96 \\
09-27-96 \\
09-27-96 \\
09-27-96 \\
09-27-96 \\
09-27-96 \\
09-27-96 \\
09-27-96 \\
09-27-96 \\
09-27-96 \\
\end{array}$ & $\begin{array}{l}11: 28 a \\
11: 28 a \\
11: 28 a \\
11: 28 a \\
11: 28 a \\
11: 28 a \\
11: 28 a \\
11: 28 a \\
11: 28 a \\
11: 28 a \\
11: 28 a \\
11: 28 a \\
\end{array}$ \\
\hline \multicolumn{5}{|c|}{ Directory of 10A-3 } & \multicolumn{5}{|c|}{21 files, 39,333 bytes totat } \\
\hline $\begin{array}{l}\text { B10A-3 } \\
\text { B10A-3 } \\
\text { B10A-3 } \\
\text { B10A-3 } \\
\text { B10A-3 } \\
\text { B10A-3 } \\
\text { B10A-3 } \\
\text { B10A-3 } \\
\text { B10A-3 } \\
\text { B10A-3 }\end{array}$ & $\begin{array}{l}1 \\
10 \\
2 \\
3 \\
4 \\
5 \\
6 \\
7 \\
8 \\
9\end{array}$ & $\begin{array}{r}984 \\
565 \\
205 \\
2,758 \\
2,748 \\
1,561 \\
1,561 \\
1,558 \\
1,558 \\
352\end{array}$ & $\begin{array}{l}09-27-96 \\
09-29-96 \\
09-27-96 \\
09-27-96 \\
09-27-96 \\
09-27-96 \\
09-27-96 \\
09-27-96 \\
09-27-96 \\
09-27-96\end{array}$ & $\begin{array}{r}11: 29 a \\
3: 43 p \\
11: 29 a \\
11: 29 a \\
11: 29 a \\
11: 29 a \\
11: 29 a \\
11: 29 a \\
11: 29 a \\
11: 29 a\end{array}$ & $\begin{array}{l}\text { E1OA-3 } \\
\text { E10A-3 } \\
\text { E10A-3 } \\
\text { E10A-3 } \\
\text { E10A-3 } \\
\text { E10A-3 } \\
\text { E10A-3 } \\
\text { E10A-3 } \\
\text { E10A-3 } \\
\text { E10A-3 } \\
\text { E10A-3 }\end{array}$ & $\begin{array}{l}1 \\
17 \\
18 \\
2 \\
3 \\
4 \\
5 \\
6 \\
7 \\
8 \\
9 \\
\end{array}$ & $\begin{array}{r}984 \\
6,101 \\
6,101 \\
205 \\
2,752 \\
2,749 \\
1,561 \\
1,561 \\
1,558 \\
1,558 \\
353 \\
\end{array}$ & $\begin{array}{l}09-27-96 \\
09-27-96 \\
09-27-96 \\
09-27-96 \\
09-27-96 \\
09-27-96 \\
09-27-96 \\
09-27-96 \\
09-27-96 \\
09-27-96 \\
09-27-96 \\
\end{array}$ & $\begin{array}{l}11: 29 a \\
11: 29 a \\
11: 29 a \\
11: 29 a \\
11: 29 a \\
11: 29 a \\
11: 29 a \\
11: 29 a \\
11: 29 a \\
11: 29 a \\
11: 29 a \\
\end{array}$ \\
\hline
\end{tabular}


Table 6. Disk Table of Contents (Sheet 2 of 2)

\begin{tabular}{|c|c|c|c|c|c|c|c|c|c|}
\hline \multicolumn{2}{|c|}{ File Name } & \multirow{2}{*}{$\begin{array}{c}\text { Size } \\
\text { (bytes) }\end{array}$} & \multicolumn{2}{|c|}{ Creation } & \multicolumn{2}{|c|}{ File Name } & \multirow{2}{*}{$\begin{array}{c}\text { size } \\
\text { (bytes) }\end{array}$} & \multicolumn{2}{|c|}{ Creation } \\
\hline Base & Ext. & & Date & Time & Base & $x t$. & & Date & Time \\
\hline \multicolumn{5}{|c|}{ Directory of 10A-4 } & \multicolumn{5}{|c|}{22 files, 40,069 bytes total } \\
\hline $\begin{array}{l}\text { B10A-4 } \\
\text { B10A-4 } \\
\text { B10A-4 } \\
810 A-4 \\
\text { B10A-4 } \\
\text { B10A-4 } \\
\text { B10A-4 } \\
\text { B10A-4 } \\
\text { B10A-4 } \\
\text { B10A-4 }\end{array}$ & $\begin{array}{l}1 \\
10 \\
2 \\
3 \\
4 \\
5 \\
6 \\
7 \\
8 \\
9\end{array}$ & $\begin{array}{r}984 \\
564 \\
205 \\
2,758 \\
2,748 \\
1,561 \\
1,561 \\
1,558 \\
1,558 \\
352\end{array}$ & $\begin{array}{l}09-27-96 \\
10-02-96 \\
09-27-96 \\
09-27-96 \\
09-27-96 \\
09-27-96 \\
09-27-96 \\
09-27-96 \\
09-27-96 \\
09-27-96\end{array}$ & $\begin{array}{r}11: 30 a \\
3: 34 \mathrm{p} \\
11: 30 \mathrm{a} \\
11: 30 \mathrm{a} \\
11: 30 \mathrm{a} \\
11: 30 \mathrm{a} \\
11: 30 \mathrm{a} \\
11: 30 \mathrm{a} \\
11: 30 \mathrm{a} \\
11: 30 \mathrm{a}\end{array}$ & $\begin{array}{l}\text { E10A- } \\
\text { E10A- } \\
\text { E10A- } \\
\text { E10A- } \\
\text { E10A- } \\
\text { E10A-4 } \\
\text { E10A-4 } \\
\text { E10A- } \\
\text { E10A- } \\
\text { E10A- } \\
\text { E10A- } \\
\text { E1OA- }\end{array}$ & $\begin{array}{l}1 \\
10 \\
17 \\
18 \\
2 \\
3 \\
4 \\
5 \\
6 \\
7 \\
8 \\
9 \\
\end{array}$ & $\begin{array}{r}984 \\
737 \\
6,101 \\
6,101 \\
205 \\
2,752 \\
2,749 \\
1,561 \\
1,561 \\
1,558 \\
1,558 \\
353\end{array}$ & $\begin{array}{l}09-27-96 \\
09-27-96 \\
09-27-96 \\
09-27-96 \\
09-27-96 \\
09-27-96 \\
09-27-96 \\
09-27-96 \\
09-27-96 \\
09-27-96 \\
09-27-96 \\
09-27-96\end{array}$ & $\begin{array}{l}11: 30 a \\
11: 30 a \\
11: 30 a \\
11: 30 a \\
11: 30 a \\
11: 30 a \\
11: 30 a \\
11: 31 a \\
11: 31 a \\
11: 31 a \\
11: 31 a \\
11: 31 a\end{array}$ \\
\hline \multicolumn{5}{|c|}{ Directory of $10 \mathrm{~B}$} & \multicolumn{5}{|c|}{22 files, 40,159 bytes total } \\
\hline $\begin{array}{l}\text { B10B } \\
810 B \\
B 10 B \\
810 B \\
B 10 B \\
B 10 B \\
B 10 B \\
B 10 B \\
B 10 B \\
B 10 B\end{array}$ & $\begin{array}{l}1 \\
10 \\
2 \\
3 \\
4 \\
5 \\
6 \\
7 \\
8 \\
9\end{array}$ & $\begin{array}{r}980 \\
733 \\
203 \\
2,754 \\
2,744 \\
1,557 \\
1,557 \\
1,554 \\
1,554 \\
348\end{array}$ & $\begin{array}{l}09-27-96 \\
09-27-96 \\
09-27-96 \\
09-27-96 \\
09-27-96 \\
09-27-96 \\
09-27-96 \\
09-27-96 \\
09-27-96 \\
09-27-96\end{array}$ & $\begin{array}{l}11: 31 a \\
11: 31 a \\
11: 31 a \\
11: 31 a \\
11: 31 a \\
11: 31 a \\
11: 31 a \\
11: 31 a \\
11: 31 a \\
11: 31 a\end{array}$ & $\begin{array}{l}\text { E10B } \\
\text { E10B } \\
\text { E10B } \\
\text { E10B } \\
\text { E10B } \\
\text { E10B } \\
\text { E10B } \\
\text { E10B } \\
\text { E10B } \\
\text { E10B } \\
\text { E10B } \\
\text { E10B }\end{array}$ & $\begin{array}{l}1 \\
10 \\
17 \\
18 \\
2 \\
3 \\
4 \\
5 \\
6 \\
7 \\
8 \\
9\end{array}$ & $\begin{array}{r}980 \\
734 \\
6,097 \\
6,097 \\
203 \\
2,748 \\
2,745 \\
1,557 \\
1,557 \\
1,554 \\
1,554 \\
349\end{array}$ & $\begin{array}{l}09-27-96 \\
10-02-96 \\
09-27-96 \\
09-27-96 \\
09-27-96 \\
09-27-96 \\
09-27-96 \\
09-27-96 \\
09-27-96 \\
09-27-96 \\
09-27-96 \\
09-27-96\end{array}$ & $\begin{array}{r}11: 31 a \\
3: 35 p \\
11: 31 a \\
11: 31 a \\
11: 31 a \\
11: 32 a \\
11: 32 a \\
11: 32 a \\
11: 32 a \\
11: 32 a \\
11: 32 a \\
11: 32 a\end{array}$ \\
\hline
\end{tabular}


Table B10A-1.1. Fission Power Generated in MFA-1, MFA-2 and Neighboring Assemblies at BOC 10A-1

\begin{tabular}{|c|c|c|c|c|}
\hline $\begin{array}{l}\text { CORE } \\
\text { POS. } \\
1506 \\
1404 \\
1405 \\
1505 \\
1507 \\
1606 \\
1607 \\
2507 \\
2405 \\
2506 \\
2508 \\
2607 \\
2608\end{array}$ & $\begin{array}{c}\star \star * * * \star \\
\text { BELOW CORE } \\
2.246 \mathrm{E}-02 \\
7.618 \mathrm{E}-02 \\
8.659 \mathrm{E}-03 \\
1.088 \mathrm{E}-02 \\
9.496 \mathrm{E}-03 \\
5.669 \mathrm{E}-03 \\
8.989 \mathrm{E}-03 \\
2.106 \mathrm{E}-02 \\
5.745 \mathrm{E}-02 \\
9.759 \mathrm{E}-03 \\
9.327 \mathrm{E}-03 \\
6.612 \mathrm{E}-03 \\
1.328 \mathrm{E}-02 \\
8.462 \mathrm{E}-02\end{array}$ & $\begin{array}{l}\text { POWER IN } \\
\text { IN CORE } \\
4.196 \mathrm{E}+00 \\
4.933 \mathrm{E}+00 \\
4.127 \mathrm{E}+00 \\
3.800 \mathrm{E}+00 \\
3.586 \mathrm{E}+00 \\
3.720 \mathrm{E}+00 \\
3.110 \mathrm{E}+00 \\
3.963 \mathrm{E}+00 \\
4.173 \mathrm{E}+00 \\
3.659 \mathrm{E}+00 \\
4.376 \mathrm{E}+00 \\
3.376 \mathrm{E}+00 \\
3.186 \mathrm{E}+00 \\
2.998 \mathrm{E}+00\end{array}$ & $\begin{array}{r}\text { MEGAWATTS } \\
\text { ABOVE CORE } \\
1.709 \mathrm{E}-02 \\
4.233 \mathrm{E}-02 \\
6.616 \mathrm{E}-03 \\
6.491 \mathrm{E}-03 \\
7.131 \mathrm{E}-03 \\
4.286 \mathrm{E}-03 \\
6.475 \mathrm{E}-03 \\
1.649 \mathrm{E}-02 \\
3.298 \mathrm{E}-02 \\
6.848 \mathrm{E}-03 \\
6.847 \mathrm{E}-03 \\
4.948 \mathrm{E}-03 \\
1.062 \mathrm{E}-02 \\
4.569 \mathrm{E}-02\end{array}$ & $\begin{array}{l}\star * * * * \\
\text { TOTAL PWR } \\
4.235 E+00 \\
5.052 E+00 \\
4.143 E+00 \\
3.818 E+00 \\
3.602 E+00 \\
3.730 E+00 \\
3.126 E+00 \\
4.001 E+00 \\
4.264 E+00 \\
3.676 E+00 \\
4.392 E+00 \\
3.387 E+00 \\
3.210 E+00 \\
3.128 E+00\end{array}$ \\
\hline
\end{tabular}

Table B1OA-1.2. Assembly Averaged Total Flux for MFA-1 and MFA-2 at BOC 10A-1

$\begin{array}{ccc} & \text { Core } & \text { Total Flux } \\ \text { Assembly } & \text { Pos. } & \begin{array}{c}\left.\text { (n/ } \mathrm{cm}^{2}-\mathrm{sec}\right) \\ \text { MFA-1 }\end{array} \\ \text { MFA-2 } & 2506 & 3.006 \mathrm{E}+15 \\ \text { MFA } & 2.836 \mathrm{E}+15\end{array}$


Table BlOA-1.3. Axial Distribution of Total Flux, Fast Flux and Power in MFA-1 at $B O C$ 10A-1

$\begin{array}{rccc}z(\mathrm{~cm}) & \text { Total Flux } & \text { Flux }>0.1 \mathrm{MeV} & \text { Power } \\ -97.78 & 2.6526 \mathrm{E}-02 & 1.3341 \mathrm{E}-02 & 0.0000 \mathrm{E}+00 \\ -92.78 & 5.7045 \mathrm{E}-02 & 2.5782 \mathrm{E}-02 & 0.0000 \mathrm{E}+00 \\ -87.78 & 9.1548 \mathrm{E}-02 & 4.1301 \mathrm{E}-02 & 0.0000 \mathrm{E}+00 \\ -82.78 & 1.3267 \mathrm{E}-01 & 6.1694 \mathrm{E}-02 & 0.0000 \mathrm{E}+00 \\ -77.78 & 1.8310 \mathrm{E}-01 & 8.9179 \mathrm{E}-02 & 0.0000 \mathrm{E}+00 \\ -72.15 & 2.4031 \mathrm{E}-01 & 1.2480 \mathrm{E}-01 & 0.0000 \mathrm{E}+00 \\ -65.90 & 2.9764 \mathrm{E}-01 & 1.6976 \mathrm{E}-01 & 0.0000 \mathrm{E}+00 \\ -60.35 & 3.5878 \mathrm{E}-01 & 2.3041 \mathrm{E}-01 & 1.6617 \mathrm{E}-02 \\ -55.49 & 4.4025 \mathrm{E}-01 & 3.1379 \mathrm{E}-01 & 2.4379 \mathrm{E}-02 \\ -50.62 & 5.3615 \mathrm{E}-01 & 4.2430 \mathrm{E}-01 & 3.7703 \mathrm{E}-02 \\ -47.17 & 6.1441 \mathrm{E}-01 & 5.3037 \mathrm{E}-01 & 5.4264 \mathrm{E}-02 \\ -43.58 & 6.9713 \mathrm{E}-01 & 6.5148 \mathrm{E}-01 & 7.3365 \mathrm{E}-01 \\ -38.45 & 8.1621 \mathrm{E}-01 & 7.9755 \mathrm{E}-01 & 8.2583 \mathrm{E}-01 \\ -33.32 & 9.2677 \mathrm{E}-01 & 9.2234 \mathrm{E}-01 & 9.2756 \mathrm{E}-01 \\ -28.20 & 1.0236 \mathrm{E}+00 & 1.0268 \mathrm{E}+00 & 1.0207 \mathrm{E}+00 \\ -23.07 & 1.1043 \mathrm{E}+00 & 1.1116 \mathrm{E}+00 & 1.0995 \mathrm{E}+00 \\ -17.94 & 1.1673 \mathrm{E}+00 & 1.1768 \mathrm{E}+00 & 1.1613 \mathrm{E}+00 \\ -12.82 & 1.2115 \mathrm{E}+00 & 1.2220 \mathrm{E}+00 & 1.2047 \mathrm{E}+00 \\ -7.69 & 1.2365 \mathrm{E}+00 & 1.2476 \mathrm{E}+00 & 1.2293 \mathrm{E}+00 \\ -2.56 & 1.2421 \mathrm{E}+00 & 1.2534 \mathrm{E}+00 & 1.2347 \mathrm{E}+00 \\ 2.56 & 1.2284 \mathrm{E}+00 & 1.2397 \mathrm{E}+00 & 1.2209 \mathrm{E}+00 \\ 7.69 & 1.1955 \mathrm{E}+00 & 1.2068 \mathrm{E}+00 & 1.1882 \mathrm{E}+00 \\ 12.82 & 1.1445 \mathrm{E}+00 & 1.1555 \mathrm{E}+00 & 1.1376 \mathrm{E}+00 \\ 17.94 & 1.0769 \mathrm{E}+00 & 1.0873 \mathrm{E}+00 & 1.0706 \mathrm{E}+00 \\ 23.07 & 9.9430 \mathrm{E}-01 & 1.0030 \mathrm{E}+00 & 9.8896 \mathrm{E}-01 \\ 28.20 & 8.9899 \mathrm{E}-01 & 9.0449 \mathrm{E}-01 & 8.9515 \mathrm{E}-01 \\ 33.32 & 7.9329 \mathrm{E}-01 & 7.9301 \mathrm{E}-01 & 7.9209 \mathrm{E}-01 \\ 38.45 & 6.7981 \mathrm{E}-01 & 6.6873 \mathrm{E}-01 & 6.8447 \mathrm{E}-01 \\ 43.58 & 5.6299 \mathrm{E}-01 & 5.3189 \mathrm{E}-01 & 5.8471 \mathrm{E}-01 \\ 47.17 & 4.8378 \mathrm{E}-01 & 4.2522 \mathrm{E}-01 & 4.3606 \mathrm{E}-02 \\ 50.62 & 4.0918 \mathrm{E}-01 & 3.3194 \mathrm{E}-01 & 2.9525 \mathrm{E}-02 \\ 55.49 & 3.1815 \mathrm{E}-01 & 2.3509 \mathrm{E}-01 & 1.8093 \mathrm{E}-02 \\ 60.35 & 2.3997 \mathrm{E}-01 & 1.6184 \mathrm{E}-01 & 1.1296 \mathrm{E}-02 \\ 64.98 & 1.9222 \mathrm{E}-01 & 1.2052 \mathrm{E}-01 & 0.0000 \mathrm{E}+00 \\ 69.39 & 1.6460 \mathrm{E}-01 & 9.8965 \mathrm{E}-02 & 0.0000 \mathrm{E}+00 \\ 76.46 & 1.2535 \mathrm{E}-01 & 7.0195 \mathrm{E}-02 & 0.0000 \mathrm{E}+00 \\ 86.80 & 8.1928 \mathrm{E}-02 & 4.2306 \mathrm{E}-02 & 0.0000 \mathrm{E}+00 \\ 98.14 & 4.7337 \mathrm{E}-02 & 2.3110 \mathrm{E}-02 & 0.0000 \mathrm{E}+00 \\ 110.00 & 2.0307 \mathrm{E}-02 & 1.0362 \mathrm{E}-02 & 0.0000 \mathrm{E}+00\end{array}$


Table B10A-1.4. Axial Distribution of Total Flux, Fast Flux and Power in MFA-2 at BOC 10A-1

$\begin{array}{rccc}\text { z }(\mathrm{cm}) & \text { Total Flux } & \text { Flux }>0.1 \text { MeV } & \text { Power } \\ -97.78 & 2.5594 \mathrm{E}-02 & 1.2927 \mathrm{E}-02 & 0.0000 \mathrm{E}+00 \\ -92.78 & 5.5013 \mathrm{E}-02 & 2.4935 \mathrm{E}-02 & 0.0000 \mathrm{E}+00 \\ -87.78 & 8.8199 \mathrm{E}-02 & 3.9859 \mathrm{E}-02 & 0.0000 \mathrm{E}+00 \\ -82.78 & 1.2771 \mathrm{E}-01 & 5.9417 \mathrm{E}-02 & 0.0000 \mathrm{E}+00 \\ -77.78 & 1.7617 \mathrm{E}-01 & 8.5771 \mathrm{E}-02 & 0.0000 \mathrm{E}+00 \\ -72.15 & 2.3120 \mathrm{E}-01 & 1.2002 \mathrm{E}-01 & 0.0000 \mathrm{E}+00 \\ -65.90 & 2.8707 \mathrm{E}-01 & 1.6387 \mathrm{E}-01 & 0.0000 \mathrm{E}+00 \\ -60.35 & 3.4727 \mathrm{E}-01 & 2.2388 \mathrm{E}-01 & 1.6431 \mathrm{E}-02 \\ -55.49 & 4.2863 \mathrm{E}-01 & 3.0663 \mathrm{E}-01 & 2.4217 \mathrm{E}-02 \\ -50.62 & 5.2581 \mathrm{E}-01 & 4.1680 \mathrm{E}-01 & 3.7476 \mathrm{E}-02 \\ -47.17 & 6.0568 \mathrm{E}-01 & 5.2258 \mathrm{E}-01 & 5.3907 \mathrm{E}-02 \\ -43.58 & 6.8985 \mathrm{E}-01 & 6.4366 \mathrm{E}-01 & 7.2306 \mathrm{E}-01 \\ -38.45 & 8.0993 \mathrm{E}-01 & 7.9061 \mathrm{E}-01 & 8.1912 \mathrm{E}-01 \\ -33.32 & 9.2109 \mathrm{E}-01 & 9.1643 \mathrm{E}-01 & 9.2189 \mathrm{E}-01 \\ -28.20 & 1.0184 \mathrm{E}+00 & 1.0219 \mathrm{E}+00 & 1.0154 \mathrm{E}+00 \\ -23.07 & 1.0999 \mathrm{E}+00 & 1.1079 \mathrm{E}+00 & 1.0949 \mathrm{E}+00 \\ -17.94 & 1.1639 \mathrm{E}+00 & 1.1743 \mathrm{E}+00 & 1.1578 \mathrm{E}+00 \\ -12.82 & 1.2092 \mathrm{E}+00 & 1.2207 \mathrm{E}+00 & 1.2025 \mathrm{E}+00 \\ -7.69 & 1.2352 \mathrm{E}+00 & 1.2471 \mathrm{E}+00 & 1.2281 \mathrm{E}+00 \\ -2.56 & 1.2415 \mathrm{E}+00 & 1.2537 \mathrm{E}+00 & 1.2342 \mathrm{E}+00 \\ 2.56 & 1.2283 \mathrm{E}+00 & 1.2406 \mathrm{E}+00 & 1.2210 \mathrm{E}+00 \\ 7.69 & 1.1963 \mathrm{E}+00 & 1.2084 \mathrm{E}+00 & 1.1892 \mathrm{E}+00 \\ 12.82 & 1.1466 \mathrm{E}+00 & 1.1583 \mathrm{E}+00 & 1.1400 \mathrm{E}+00 \\ 17.94 & 1.0806 \mathrm{E}+00 & 1.0913 \mathrm{E}+00 & 1.0748 \mathrm{E}+00 \\ 23.07 & 9.9947 \mathrm{E}-01 & 1.0081 \mathrm{E}+00 & 9.9473 \mathrm{E}-01 \\ 28.20 & 9.0506 \mathrm{E}-01 & 9.1006 \mathrm{E}-01 & 9.0196 \mathrm{E}-01 \\ 33.32 & 7.9976 \mathrm{E}-01 & 7.9844 \mathrm{E}-01 & 7.9937 \mathrm{E}-01 \\ 38.45 & 6.8613 \mathrm{E}-01 & 6.7337 \mathrm{E}-01 & 6.9155 \mathrm{E}-01 \\ 43.58 & 5.6869 \mathrm{E}-01 & 5.3526 \mathrm{E}-01 & 5.9037 \mathrm{E}-01 \\ 47.17 & 4.8861 \mathrm{E}-01 & 4.2753 \mathrm{E}-01 & 4.4266 \mathrm{E}-02 \\ 50.62 & 4.1312 \mathrm{E}-01 & 3.3359 \mathrm{E}-01 & 3.0062 \mathrm{E}-02 \\ 55.49 & 3.2164 \mathrm{E}-01 & 2.3629 \mathrm{E}-01 & 1.8544 \mathrm{E}-02 \\ 60.35 & 2.4413 \mathrm{E}-01 & 1.6316 \mathrm{E}-01 & 1.1680 \mathrm{E}-02 \\ 64.98 & 1.9720 \mathrm{E}-01 & 1.2185 \mathrm{E}-01 & 0.0000 \mathrm{E}+00 \\ 69.39 & 1.6933 \mathrm{E}-01 & 1.0004 \mathrm{E}-01 & 0.0000 \mathrm{E}+00 \\ 76.46 & 1.2922 \mathrm{E}-01 & 7.0908 \mathrm{E}-02 & 0.0000 \mathrm{E}+00 \\ 86.80 & 8.4577 \mathrm{E}-02 & 4.2724 \mathrm{E}-02 & 0.0000 \mathrm{E}+00 \\ 98.14 & 4.8841 \mathrm{E}-02 & 2.3310 \mathrm{E}-02 & 0.0000 \mathrm{E}+00 \\ 110.00 & 2.0742 \mathrm{E}-02 & 1.0361 \mathrm{E}-02 & 0.0000 \mathrm{E}+00\end{array}$


Table B10A-1.5. Fission Power Distribution by Pin in MFA-1 at BOC 10A-1

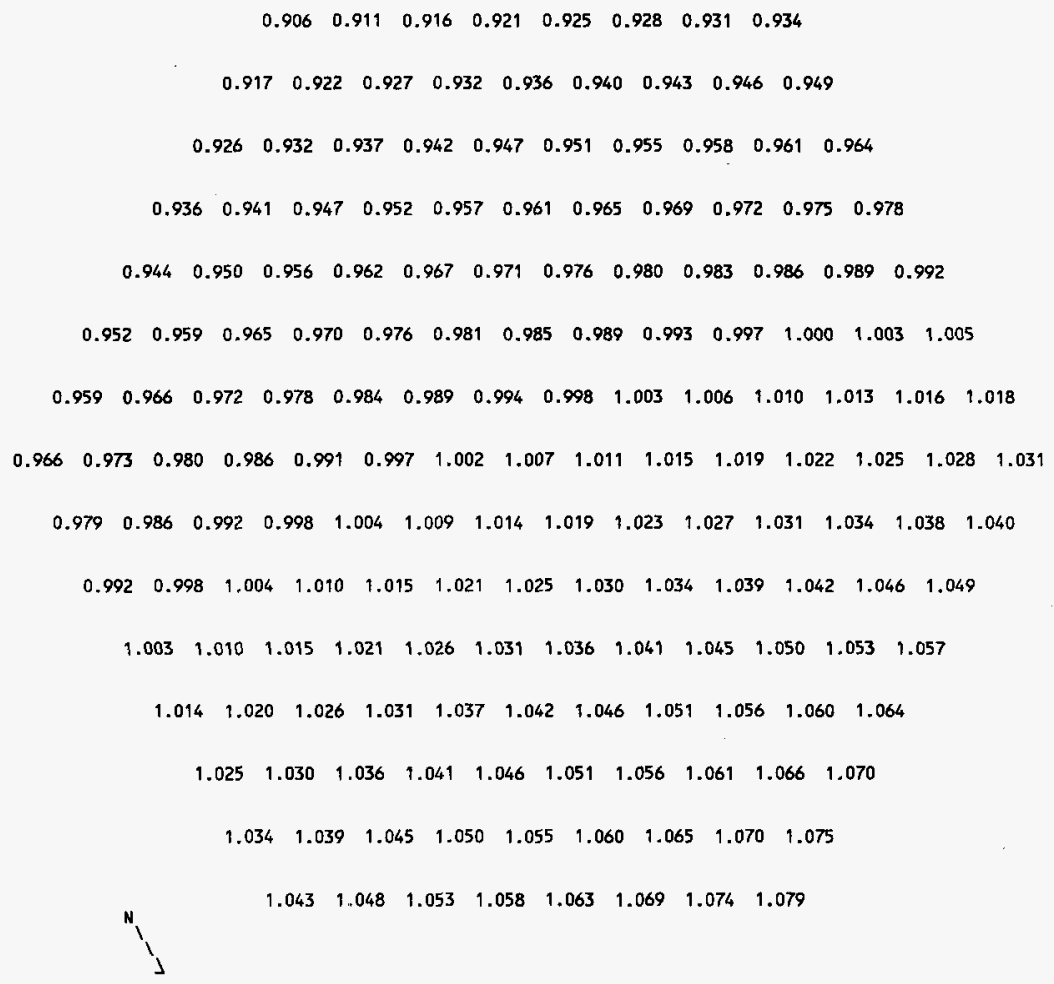


WHC-SD-FF-ANAL-009 REV 0

Table B10A-1.6. Fission Power Distribution by Pin in MFA-2 at BOC 10A-1

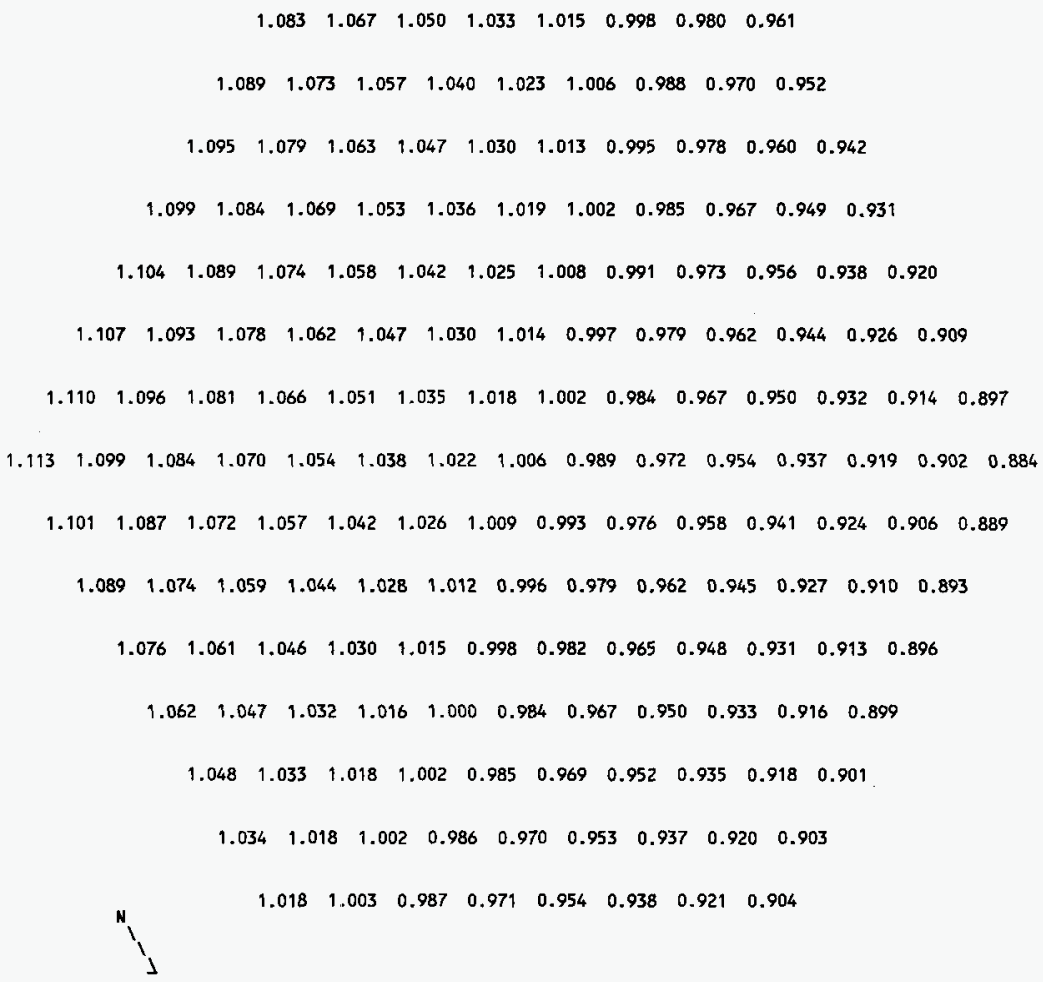


WHC-SD-FF-ANAL-009 REV 0

Table B10A-1.7. Total Flux Distribution by Pin in MFA-1 at BOC 10A-1

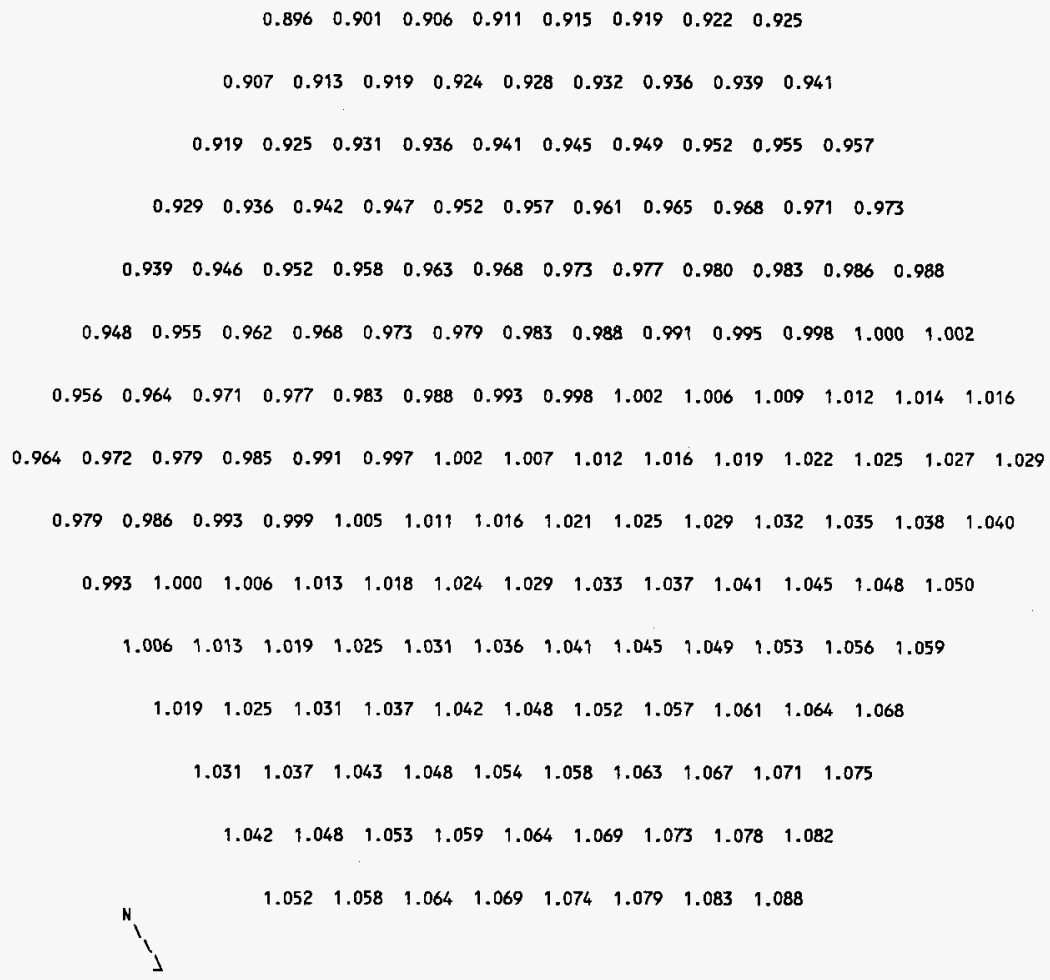


Table B10A-1.8. Total Flux Distribution by Pin in MFA-2 at BOC 10A-1

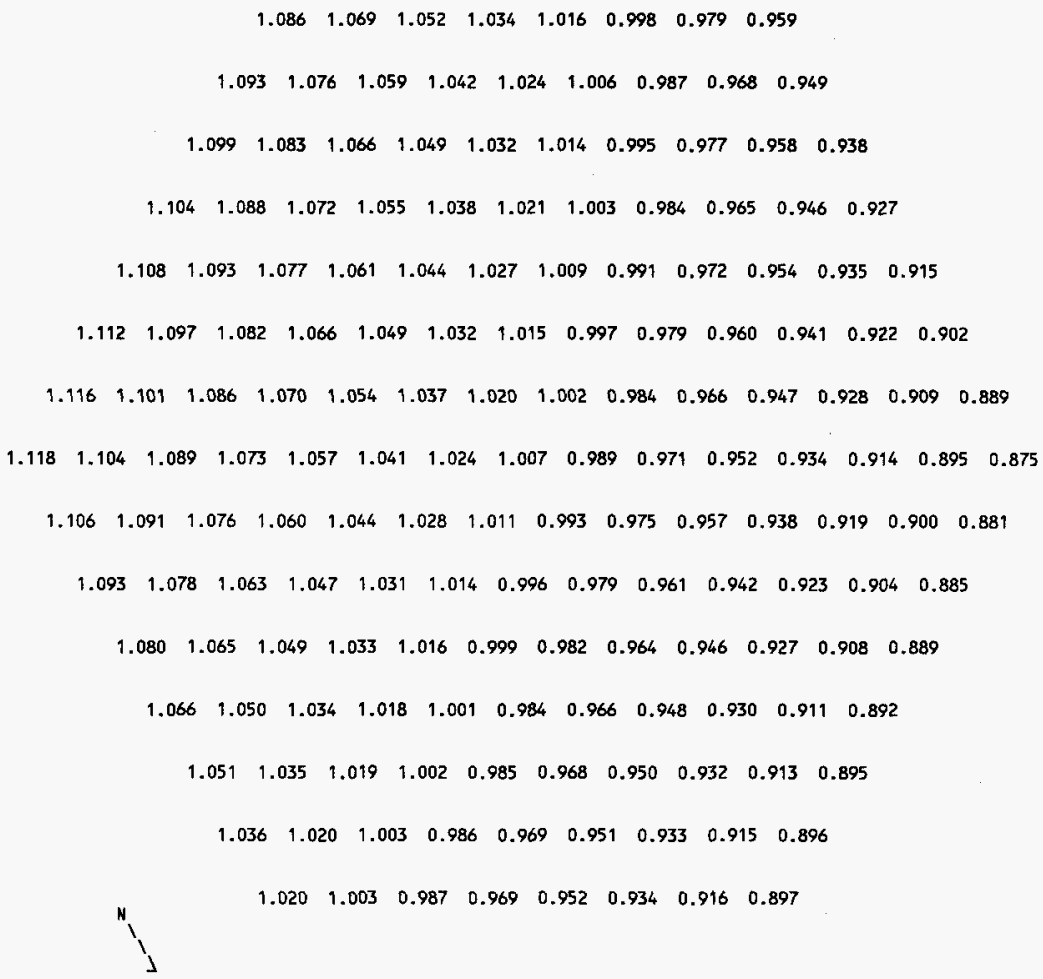


Table B10A-1.9. MFA-1 and MFA-2 Duct Wall Flux Greater Than $0.1 \mathrm{MeV}$ at $\mathrm{BOC}$ 10A-1

$\begin{array}{ccc}\text { SIDE } & \begin{array}{c}\text { FLUX }>0.1 \\ \text { MFA-1 }\end{array} & \begin{array}{c}\text { MEV } \\ \left(\mathrm{n} / \mathrm{cm}^{2}-\mathrm{sec}\right) \\ \text { MFA-1 }\end{array} \\ \text { E } & 1.9719 \mathrm{E}+15 & 1.9751 \mathrm{E}+15 \\ \text { SE } & 1.7724 \mathrm{E}+15 & 2.0325 \mathrm{E}+15 \\ \text { SW } & 1.7082 \mathrm{E}+15 & 1.8566 \mathrm{E}+15 \\ \text { W } & 1.8752 \mathrm{E}+15 & 1.6328 \mathrm{E}+15 \\ \text { NW } & 2.0845 \mathrm{E}+15 & 1.5643 \mathrm{E}+15 \\ \text { NE } & 2.1367 \mathrm{E}+15 & 1.7117 \mathrm{E}+15\end{array}$

Table B10A-1.10. Assembly 0utlet Temperatures and Flow Rates at BOC 10A-1

$\begin{array}{lccc}\text { CORE } & \text { OUTLET TEMP. } & \text { (DEG F) } & \text { FLOW RATE } \\ \text { POS. } & \text { MEASURED } & \text { CALCULATED } & \text { (LB/H) } \\ 1506 & 1054 & 1085 & 116330 \\ 1404 & 927 & 925 & 228160 \\ 1405 & 901 & 901 & 210640 \\ 1505 & 893 & 881 & 210640 \\ 1507 & 884 & 872 & 210640 \\ 1606 & 915 & 916 & 172740 \\ 1607 & 882 & 883 & 172740 \\ 2507 & 1049 & 1058 & 116330 \\ 2405 & 929 & 918 & 194730 \\ 2506 & 891 & 879 & 200300 \\ 2508 & 920 & 906 & 210640 \\ 2607 & 914 & 899 & 165660 \\ 2608 & 1078 & 1056 & 89760 \\ 2609 & 892 & 883 & 175430\end{array}$


Table B1OA-1.11. MFA-1 Sodium Subchannel Temperatures at Top of Core Elevation for BOC 10A-1

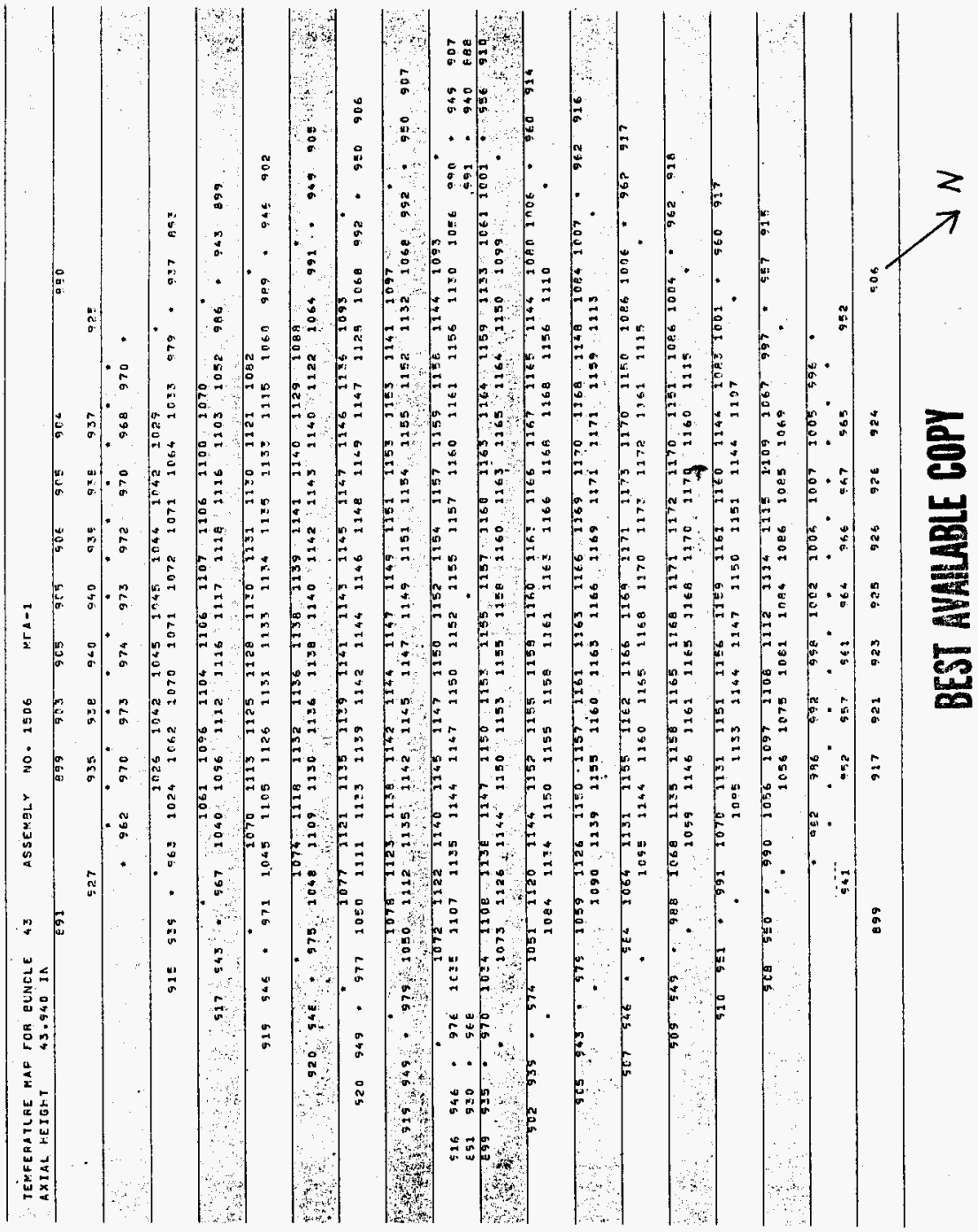


Table B10A-1.12. MFA-2 Sodium Subchannel Temperatures at Top of Core Elevation for BOC 10A-1

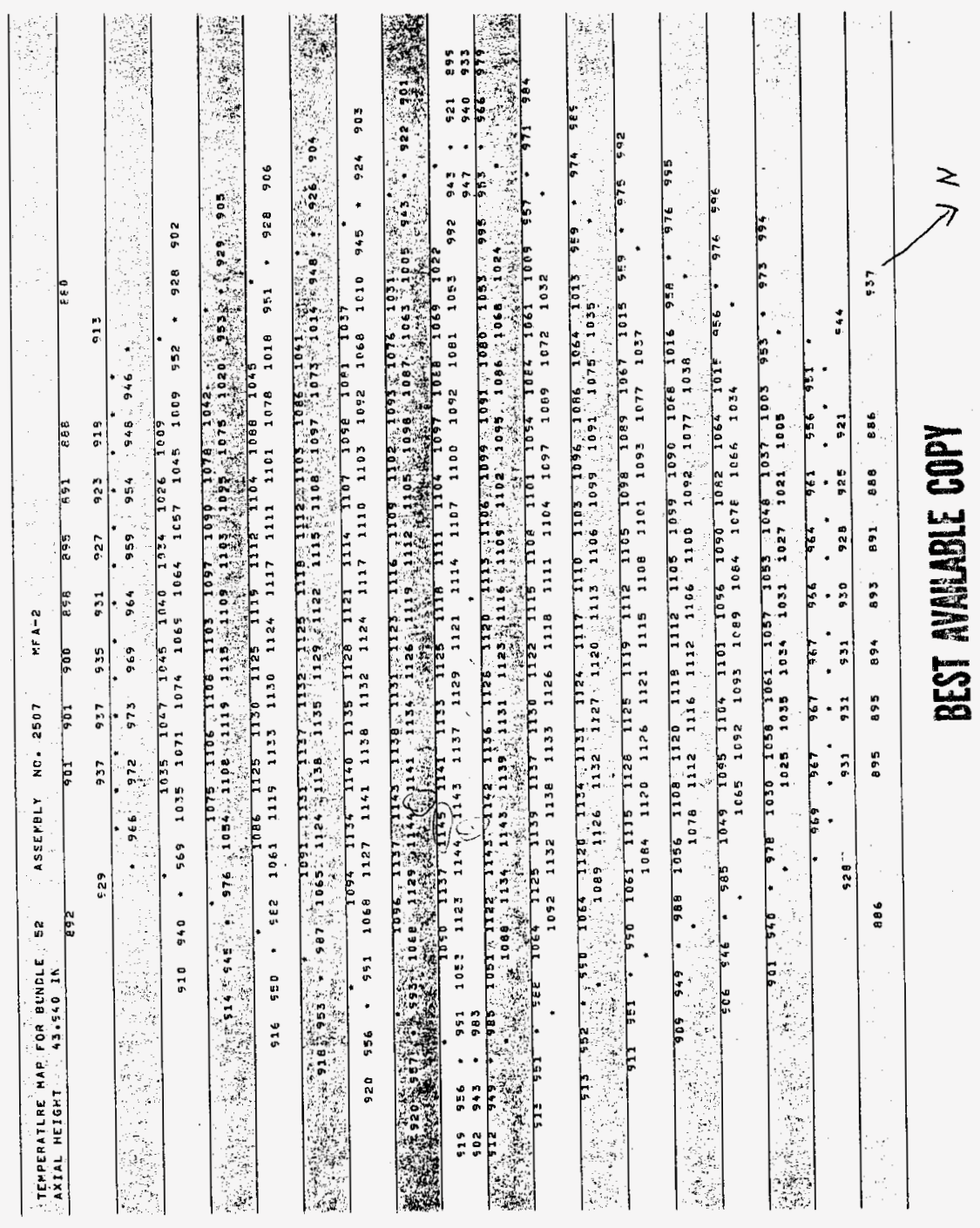


Table B10A-1.13. MFA-1 Sodium Subchannel Temperatures at Elevation of Upper Axial Blanket for BOC IOA-1

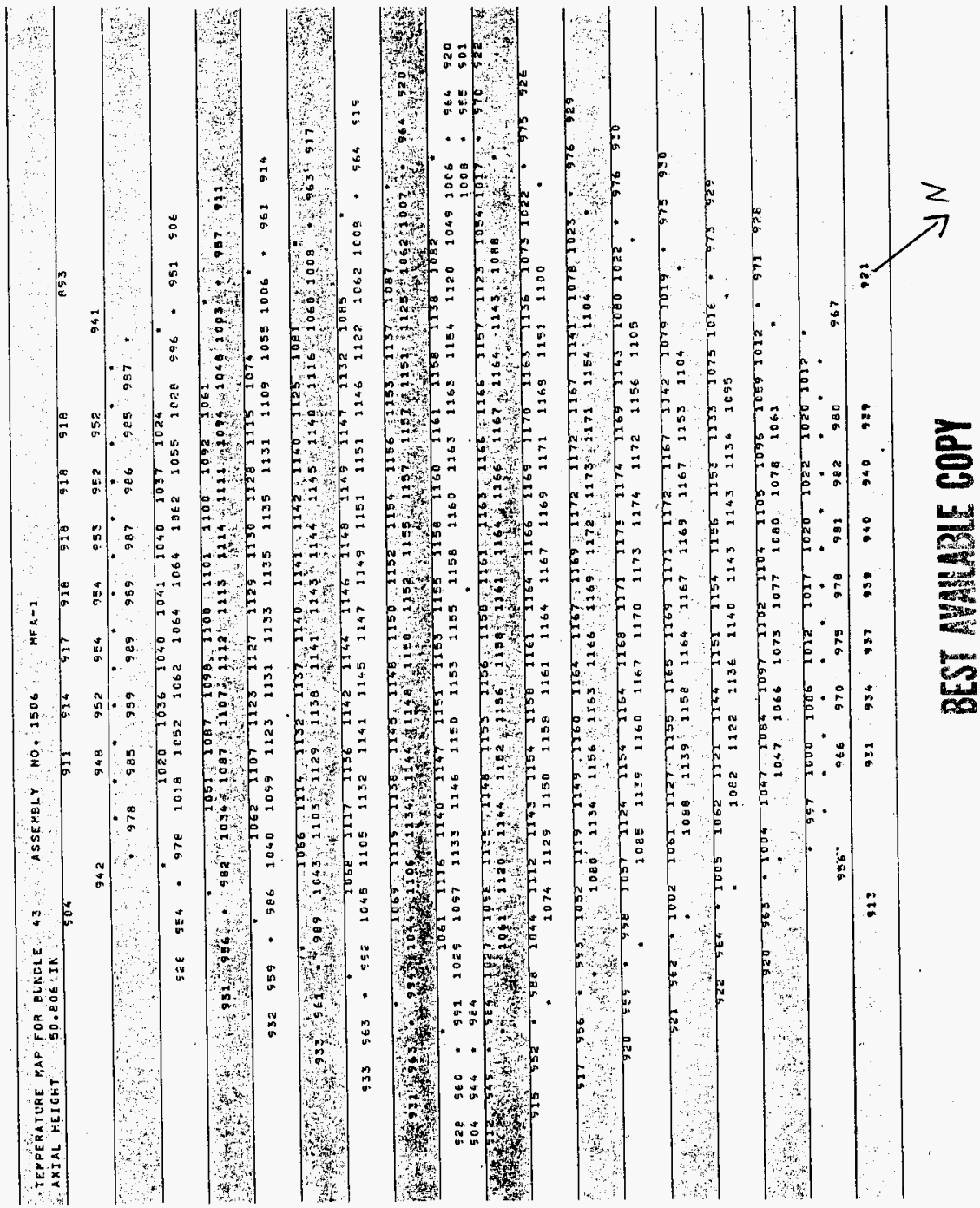


Table B10A-1.14. MFA-2 Sodium Subchannel Temperatures at Elevation of Upper Axial Blanket for BOC 10A-1

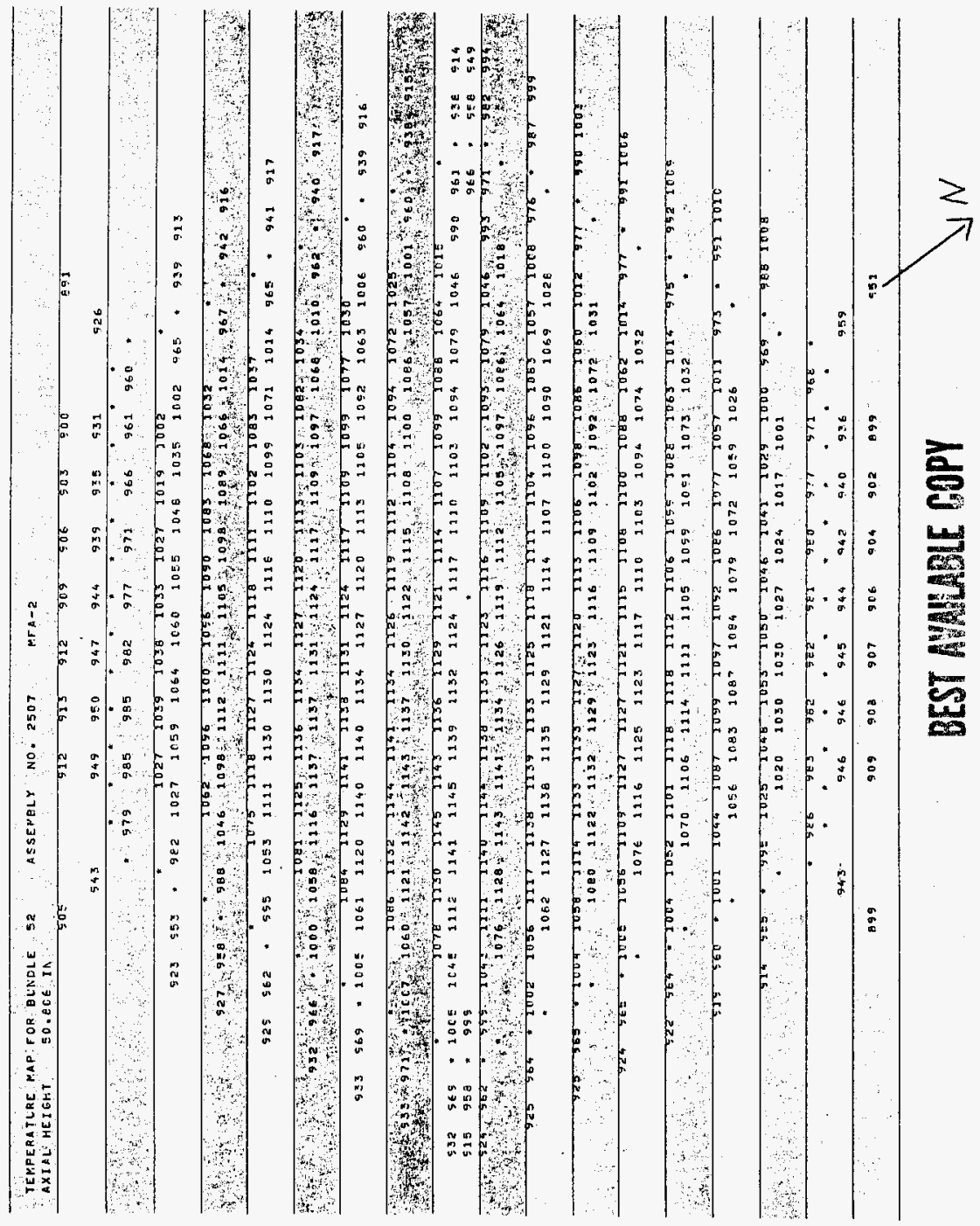


Table B10A-1.15. MFA-1 Sodium Subchannel Temperatures at Top of Fuel Pin Bundle for BOC 10A-1

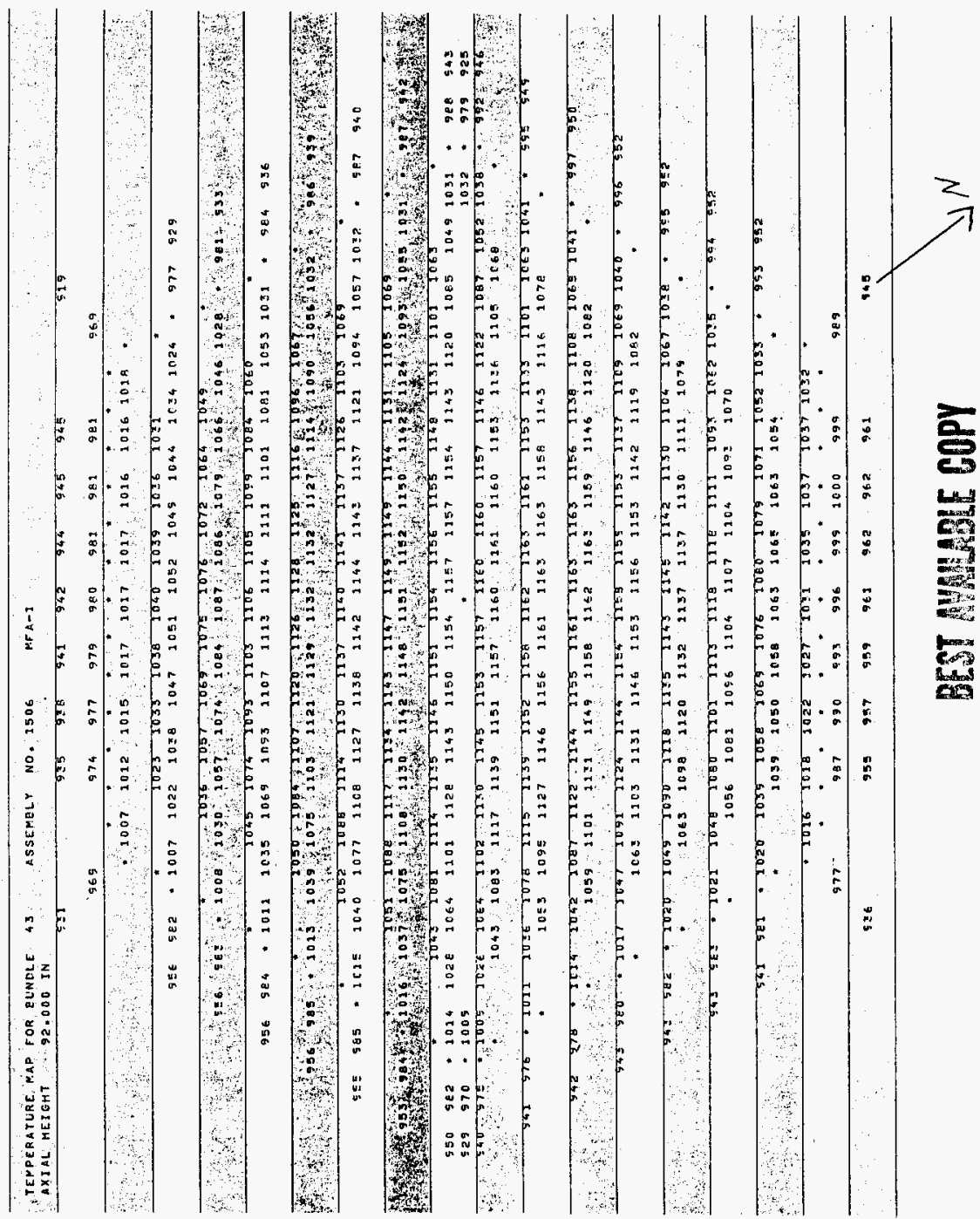


Table B10A-1.16. MFA-2 Sodium Subchannel Temperatures at Top of Fuel Pin Bundle for BOC 10A-1

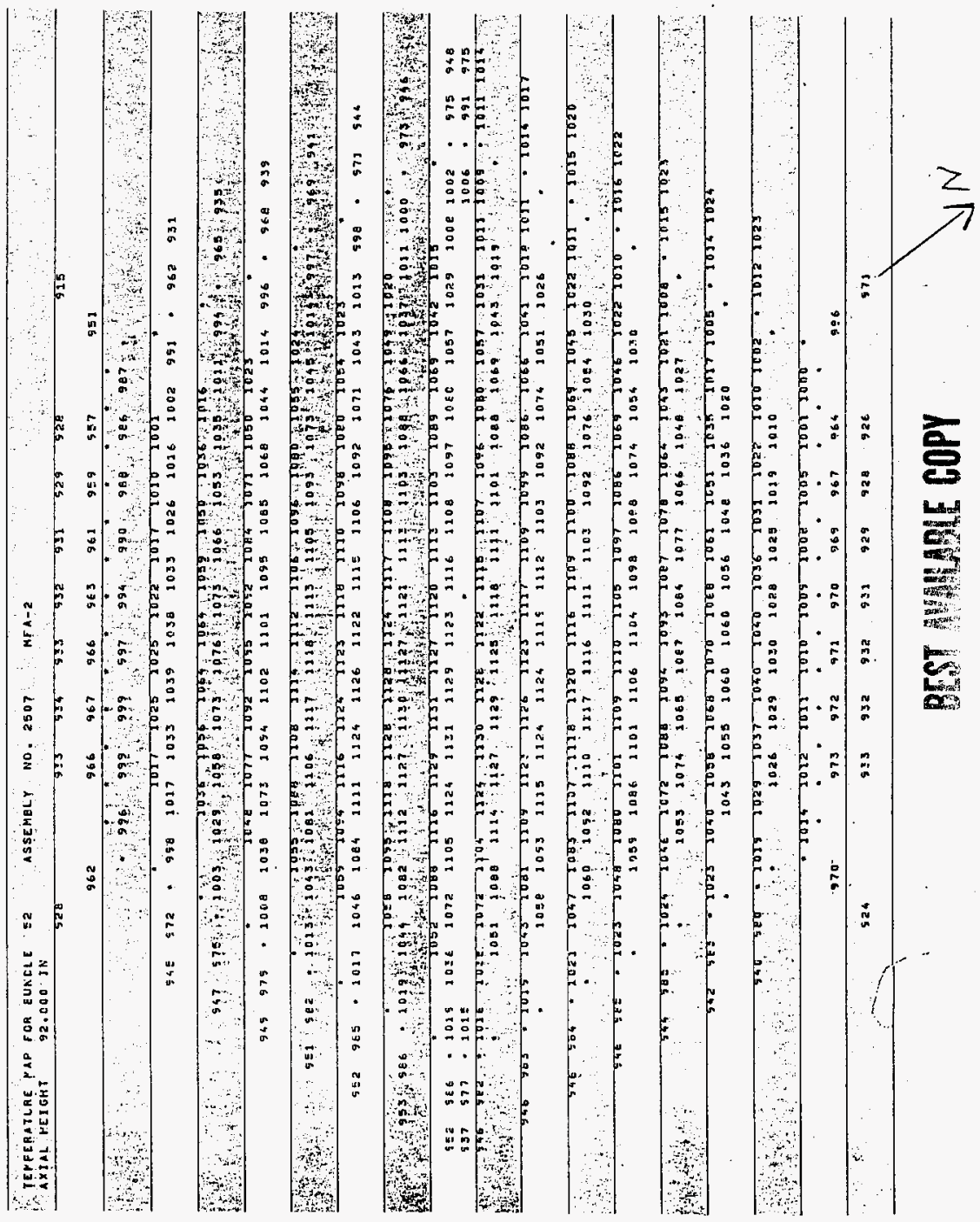


Table ElOA-1.1. Fission Power Generated in MFA-1, MFA-2 and Neighboring Assemblies at EOC 10A-1

\begin{tabular}{|c|c|c|c|c|}
\hline $\begin{array}{l}1506 \\
1404\end{array}$ & $\begin{array}{l}\star \star \star \star \star \star \star \\
\text { BELOW CORE } \\
2.758 \mathrm{E}-02 \\
8.078 \mathrm{E}-02 \\
9.261 \mathrm{E}-03 \\
1.121 \mathrm{E}-02 \\
9.750 \mathrm{E}-03 \\
6.117 \mathrm{E}-03 \\
9.165 \mathrm{E}-03 \\
2.522 \mathrm{E}-02 \\
6.120 \mathrm{E}-02 \\
1.013 \mathrm{E}-02 \\
9.791 \mathrm{E}-03 \\
6.923 \mathrm{E}-03 \\
1.574 \mathrm{E}-02\end{array}$ & $\begin{array}{l}\text { POWER IN } \\
\text { IN CORE } \\
4.106 \mathrm{E}+00 \\
4.846 \mathrm{E}+00 \\
4.037 \mathrm{E}+00 \\
3.795 \mathrm{E}+00 \\
3.510 \mathrm{E}+00\end{array}$ & $\begin{array}{l}\text { RE } \\
02 \\
02 \\
03 \\
03 \\
03 \\
03 \\
03 \\
02 \\
02 \\
03 \\
03 \\
03 \\
02\end{array}$ & $\begin{array}{l}\star \star \star * * * \\
\text { TOTAL PWR } \\
4.154 E+00 \\
4.975 E+00 \\
4.054 E+00 \\
3.814 E+00 \\
3.528 E+00 \\
3.703 E+00 \\
3.079 E+00 \\
3.925 E+00\end{array}$ \\
\hline
\end{tabular}

Table ElOA-1.2. Assembly Averaged Total Flux for MFA-1 and MFA-2 at EOC 10A-1

$\begin{array}{ccc} & \text { Core } & \text { Tota] Flux } \\ \text { Assembly } & \text { Pos. } & \left(\mathrm{n} / \mathrm{cm}^{2}-\mathrm{sec}\right) \\ \text { MFA-1 } & 1506 & 3.013 \mathrm{E}+15 \\ \text { MFA-2 } & 2507 & 2.840 E+15\end{array}$


Table ElOA-1.3. Axial Distribution of Tota1 Flux, Fast Flux and Power in MFA-1 at EOC 10A-1

$\begin{array}{rccc}\text { z }(\mathrm{cm}) & \text { Total Flux } & \text { Flux }>0.1 \mathrm{MeV} & \text { Power } \\ -97.78 & 2.5837 \mathrm{E}-02 & 1.3075 \mathrm{E}-02 & 0.0000 \mathrm{E}+00 \\ -92.78 & 5.5558 \mathrm{E}-02 & 2.5263 \mathrm{E}-02 & 0.0000 \mathrm{E}+00 \\ -87.78 & 8.9146 \mathrm{E}-02 & 4.0461 \mathrm{E}-02 & 0.0000 \mathrm{E}+00 \\ -82.78 & 1.2917 \mathrm{E}-01 & 6.0424 \mathrm{E}-02 & 0.0000 \mathrm{E}+00 \\ -77.78 & 1.7822 \mathrm{E}-01 & 8.7325 \mathrm{E}-02 & 0.0000 \mathrm{E}+00 \\ -72.15 & 2.3384 \mathrm{E}-01 & 1.2218 \mathrm{E}-01 & 0.0000 \mathrm{E}+00 \\ -65.90 & 2.8951 \mathrm{E}-01 & 1.6617 \mathrm{E}-01 & 0.0000 \mathrm{E}+00 \\ -60.35 & 3.4874 \mathrm{E}-01 & 2.2560 \mathrm{E}-01 & 2.3875 \mathrm{E}-02 \\ -55.49 & 4.2772 \mathrm{E}-01 & 3.0667 \mathrm{E}-01 & 3.2031 \mathrm{E}-02 \\ -50.62 & 5.2060 \mathrm{E}-01 & 4.1369 \mathrm{E}-01 & 4.5485 \mathrm{E}-02 \\ -47.17 & 5.9628 \mathrm{E}-01 & 5.1612 \mathrm{E}-01 & 6.1785 \mathrm{E}-02 \\ -43.58 & 6.7629 \mathrm{E}-01 & 6.3292 \mathrm{E}-01 & 7.1450 \mathrm{E}-01 \\ -38.45 & 7.9201 \mathrm{E}-01 & 7.7461 \mathrm{E}-01 & 8.0485 \mathrm{E}-01 \\ -33.32 & 9.0008 \mathrm{E}-01 & 8.9622 \mathrm{E}-01 & 9.0491 \mathrm{E}-01 \\ -28.20 & 9.9561 \mathrm{E}-01 & 9.9876 \mathrm{E}-01 & 9.9138 \mathrm{E}-01 \\ -23.07 & 1.0766 \mathrm{E}+00 & 1.0835 \mathrm{E}+00 & 1.0704 \mathrm{E}+00 \\ -17.94 & 1.1414 \mathrm{E}+00 & 1.1503 \mathrm{E}+00 & 1.1339 \mathrm{E}+00 \\ -12.82 & 1.1891 \mathrm{E}+00 & 1.1987 \mathrm{E}+00 & 1.1773 \mathrm{E}+00 \\ -7.69 & 1.2194 \mathrm{E}+00 & 1.2295 \mathrm{E}+00 & 1.2070 \mathrm{E}+00 \\ -2.56 & 1.2319 \mathrm{E}+00 & 1.2423 \mathrm{E}+00 & 1.2193 \mathrm{E}+00 \\ 2.56 & 1.2264 \mathrm{E}+00 & 1.2370 \mathrm{E}+00 & 1.2148 \mathrm{E}+00 \\ 7.69 & 1.2026 \mathrm{E}+00 & 1.2134 \mathrm{E}+00 & 1.1913 \mathrm{E}+00 \\ 12.82 & 1.1608 \mathrm{E}+00 & 1.1717 \mathrm{E}+00 & 1.1501 \mathrm{E}+00 \\ 17.94 & 1.1015 \mathrm{E}+00 & 1.1121 \mathrm{E}+00 & 1.0966 \mathrm{E}+00 \\ 23.07 & 1.0249 \mathrm{E}+00 & 1.0341 \mathrm{E}+00 & 1.0209 \mathrm{E}+00 \\ 28.20 & 9.3261 \mathrm{E}-01 & 9.3866 \mathrm{E}-01 & 9.2994 \mathrm{E}-01 \\ 33.32 & 8.2702 \mathrm{E}-01 & 8.2732 \mathrm{E}-01 & 8.3248 \mathrm{E}-01 \\ 38.45 & 7.1124 \mathrm{E}-01 & 7.0030 \mathrm{E}-01 & 7.2195 \mathrm{E}-01 \\ 43.58 & 5.9066 \mathrm{E}-01 & 5.5866 \mathrm{E}-01 & 6.1844 \mathrm{E}-01 \\ 47.17 & 5.0845 \mathrm{E}-01 & 4.4762 \mathrm{E}-01 & 5.1239 \mathrm{E}-02 \\ 50.62 & 4.3082 \mathrm{E}-01 & 3.5033 \mathrm{E}-01 & 3.6074 \mathrm{E}-02 \\ 55.49 & 3.3565 \mathrm{E}-01 & 2.4883 \mathrm{E}-01 & 2.3419 \mathrm{E}-02 \\ 60.35 & 2.5367 \mathrm{E}-01 & 1.7175 \mathrm{E}-01 & 1.5577 \mathrm{E}-02 \\ 64.98 & 2.0357 \mathrm{E}-01 & 1.2806 \mathrm{E}-01 & 0.0000 \mathrm{E}+00 \\ 69.39 & 1.7450 \mathrm{E}-01 & 1.0524 \mathrm{E}-01 & 0.0000 \mathrm{E}+00 \\ 76.46 & 1.3306 \mathrm{E}-01 & 7.4740 \mathrm{E}-02 & 0.0000 \mathrm{E}+00 \\ 86.80 & 8.7042 \mathrm{E}-02 & 4.5095 \mathrm{E}-02 & 0.0000 \mathrm{E}+00 \\ 98.14 & 5.0290 \mathrm{E}-02 & 2.4643 \mathrm{E}-02 & 0.0000 \mathrm{E}+00 \\ 110.00 & 2.1534 \mathrm{E}-02 & 1.1037 \mathrm{E}-02 & 0.0000 \mathrm{E}+00\end{array}$


Table E10A-1.4. Axial Distribution of Total Flux, Fast Flux and Power in MFA-2 at EOC 10A-1

$\begin{array}{rccc}\text { z }(\mathrm{cm}) & \text { Total Flux } & \text { Flux }>0.1 \mathrm{MeV} & \text { Power } \\ -97.78 & 2.5014 \mathrm{E}-02 & 1.2714 \mathrm{E}-02 & 0.0000 \mathrm{E}+00 \\ -92.78 & 5.3755 \mathrm{E}-02 & 2.4520 \mathrm{E}-02 & 0.0000 \mathrm{E}+00 \\ -87.78 & 8.6170 \mathrm{E}-02 & 3.9187 \mathrm{E}-02 & 0.0000 \mathrm{E}+00 \\ -82.78 & 1.2475 \mathrm{E}-01 & 5.8402 \mathrm{E}-02 & 0.0000 \mathrm{E}+00 \\ -77.78 & 1.7203 \mathrm{E}-01 & 8.4284 \mathrm{E}-02 & 0.0000 \mathrm{E}+00 \\ -72.15 & 2.2570 \mathrm{E}-01 & 1.1791 \mathrm{E}-01 & 0.0000 \mathrm{E}+00 \\ -65.90 & 2.8013 \mathrm{E}-01 & 1.6097 \mathrm{E}-01 & 0.0000 \mathrm{E}+00 \\ -60.35 & 3.3871 \mathrm{E}-01 & 2.1998 \mathrm{E}-01 & 2.2643 \mathrm{E}-02 \\ -55.49 & 4.1782 \mathrm{E}-01 & 3.0072 \mathrm{E}-01 & 3.0807 \mathrm{E}-02 \\ -50.62 & 5.1230 \mathrm{E}-01 & 4.0778 \mathrm{E}-01 & 4.4321 \mathrm{E}-02 \\ -47.17 & 5.8983 \mathrm{E}-01 & 5.1029 \mathrm{E}-01 & 6.0658 \mathrm{E}-02 \\ -43.58 & 6.7155 \mathrm{E}-01 & 6.2756 \mathrm{E}-01 & 7.0665 \mathrm{E}-01 \\ -38.45 & 7.8864 \mathrm{E}-01 & 7.7056 \mathrm{E}-01 & 8.0111 \mathrm{E}-01 \\ -33.32 & 8.9759 \mathrm{E}-01 & 8.9354 \mathrm{E}-01 & 9.0244 \mathrm{E}-01 \\ -28.20 & 9.9383 \mathrm{E}-01 & 9.9728 \mathrm{E}-01 & 9.8976 \mathrm{E}-01 \\ -23.07 & 1.0756 \mathrm{E}+00 & 1.0832 \mathrm{E}+00 & 1.0694 \mathrm{E}+00 \\ -17.94 & 1.1414 \mathrm{E}+00 & 1.1511 \mathrm{E}+00 & 1.1340 \mathrm{E}+00 \\ -12.82 & 1.1900 \mathrm{E}+00 & 1.2005 \mathrm{E}+00 & 1.1786 \mathrm{E}+00 \\ -7.69 & 1.2208 \mathrm{E}+00 & 1.2317 \mathrm{E}+00 & 1.2088 \mathrm{E}+00 \\ -2.56 & 1.2332 \mathrm{E}+00 & 1.2444 \mathrm{E}+00 & 1.2210 \mathrm{E}+00 \\ 2.56 & 1.2271 \mathrm{E}+00 & 1.2386 \mathrm{E}+00 & 1.2158 \mathrm{E}+00 \\ 7.69 & 1.2027 \mathrm{E}+00 & 1.2143 \mathrm{E}+00 & 1.1916 \mathrm{E}+00 \\ 12.82 & 1.1604 \mathrm{E}+00 & 1.1718 \mathrm{E}+00 & 1.1500 \mathrm{E}+00 \\ 17.94 & 1.1010 \mathrm{E}+00 & 1.1119 \mathrm{E}+00 & 1.0962 \mathrm{E}+00 \\ 23.07 & 1.0249 \mathrm{E}+00 & 1.0339 \mathrm{E}+00 & 1.0211 \mathrm{E}+00 \\ 28.20 & 9.3348 \mathrm{E}-01 & 9.3904 \mathrm{E}-01 & 9.3126 \mathrm{E}-01 \\ 33.32 & 8.2917 \mathrm{E}-01 & 8.2842 \mathrm{E}-01 & 8.3497 \mathrm{E}-01 \\ 38.45 & 7.1444 \mathrm{E}-01 & 7.0185 \mathrm{E}-01 & 7.2553 \mathrm{E}-01 \\ 43.58 & 5.9443 \mathrm{E}-01 & 5.6016 \mathrm{E}-01 & 6.2175 \mathrm{E}-01 \\ 47.17 & 5.1195 \mathrm{E}-01 & 4.4868 \mathrm{E}-01 & 5.1566 \mathrm{E}-02 \\ 50.62 & 4.3397 \mathrm{E}-01 & 3.5131 \mathrm{E}-01 & 3.6332 \mathrm{E}-02 \\ 55.49 & 3.3891 \mathrm{E}-01 & 2.4986 \mathrm{E}-01 & 2.3653 \mathrm{E}-02 \\ 60.35 & 2.5801 \mathrm{E}-01 & 1.7319 \mathrm{E}-01 & 1.5841 \mathrm{E}-02 \\ 64.98 & 2.0899 \mathrm{E}-01 & 1.2963 \mathrm{E}-01 & 0.0000 \mathrm{E}+00 \\ 69.39 & 1.7972 \mathrm{E}-01 & 1.0657 \mathrm{E}-01 & 0.0000 \mathrm{E}+00 \\ 76.46 & 1.3742 \mathrm{E}-01 & 7.5673 \mathrm{E}-02 & 0.0000 \mathrm{E}+00 \\ 86.80 & 9.0081 \mathrm{E}-02 & 4.5673 \mathrm{E}-02 & 0.0000 \mathrm{E}+00 \\ 98.14 & 5.2051 \mathrm{E}-02 & 2.4941 \mathrm{E}-02 & 0.0000 \mathrm{E}+00 \\ 110.00 & 2.2096 \mathrm{E}-02 & 1.1084 \mathrm{E}-02 & 0.0000 \mathrm{E}+00\end{array}$




\section{WHC-SD-FF-ANAL-009 REV 0}

Table E1OA-1.5. Fission Power Distribution by Pin in MFA-1 at EOC 10A-1

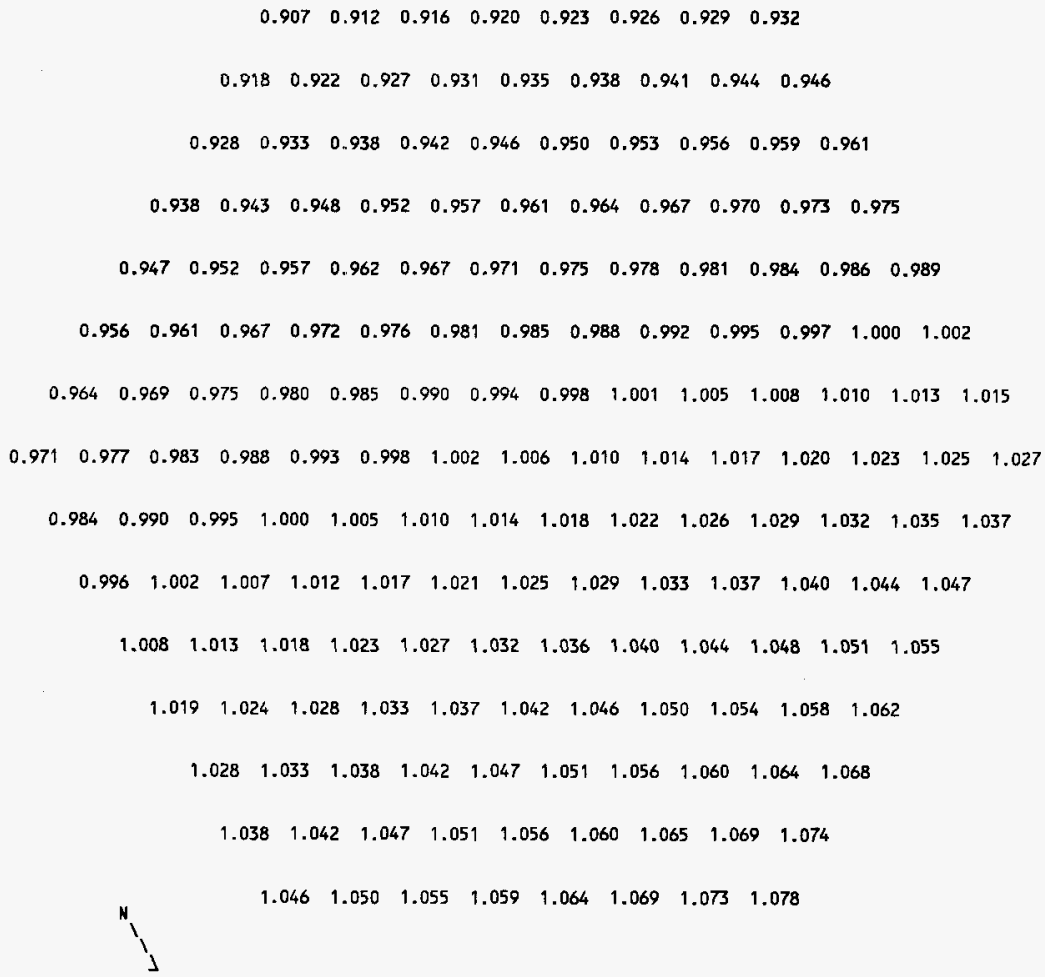


Table ElOA-1.6. Fission Power Distribution by Pin in MFA-2 at EOC 1OA-1

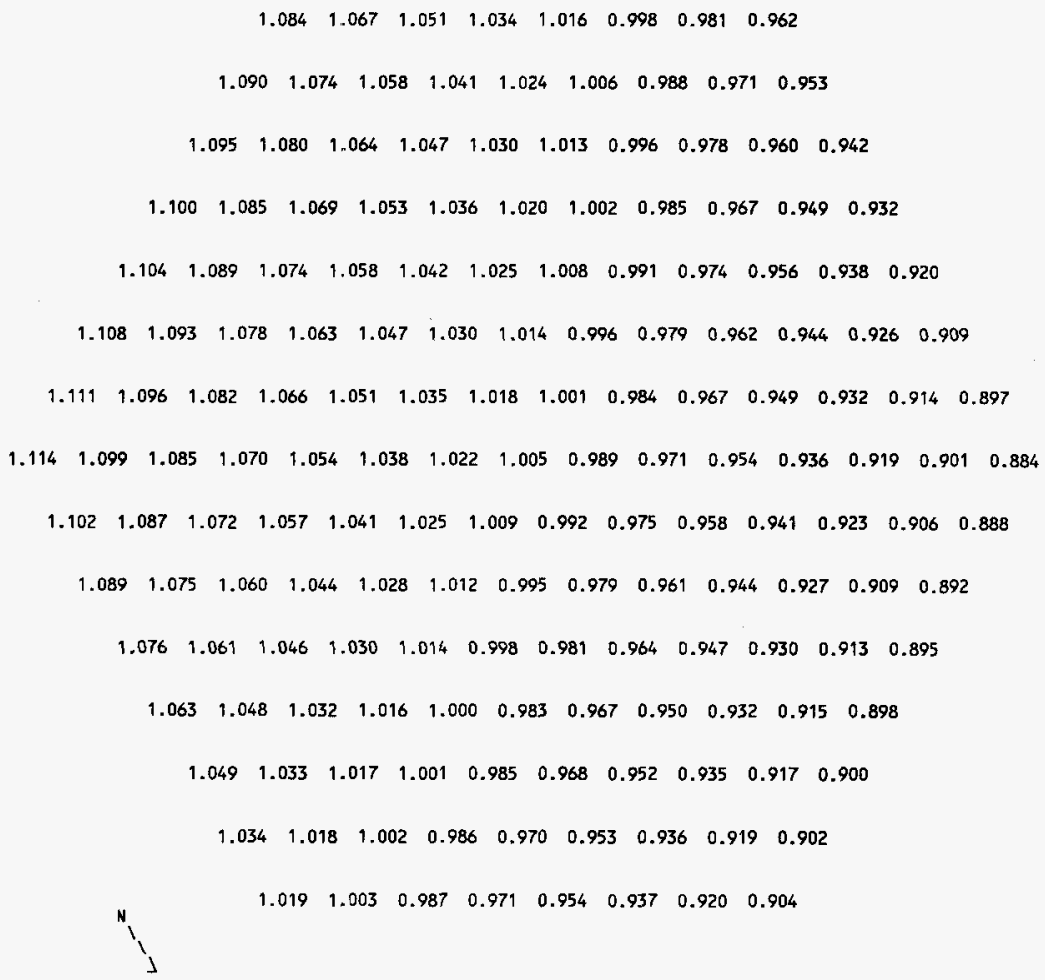




\section{WHC-SD-FF-ANAL-009 REV 0}

Table E10A-1.7. Total Flux Distribution by Pin in MFA-1 at EOC 10A-1

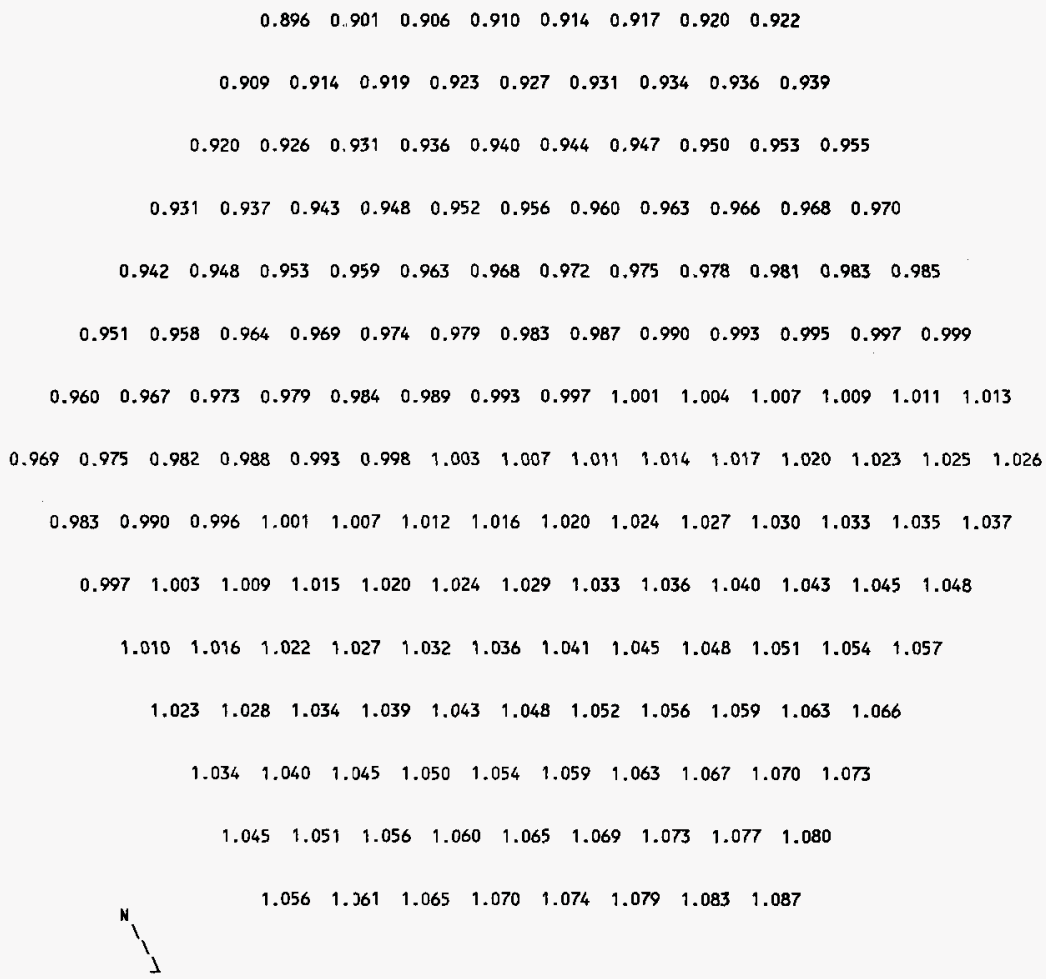


Table E1OA-1.8. Total Flux Distribution by Pin in MFA-2 at EOC 10A-1

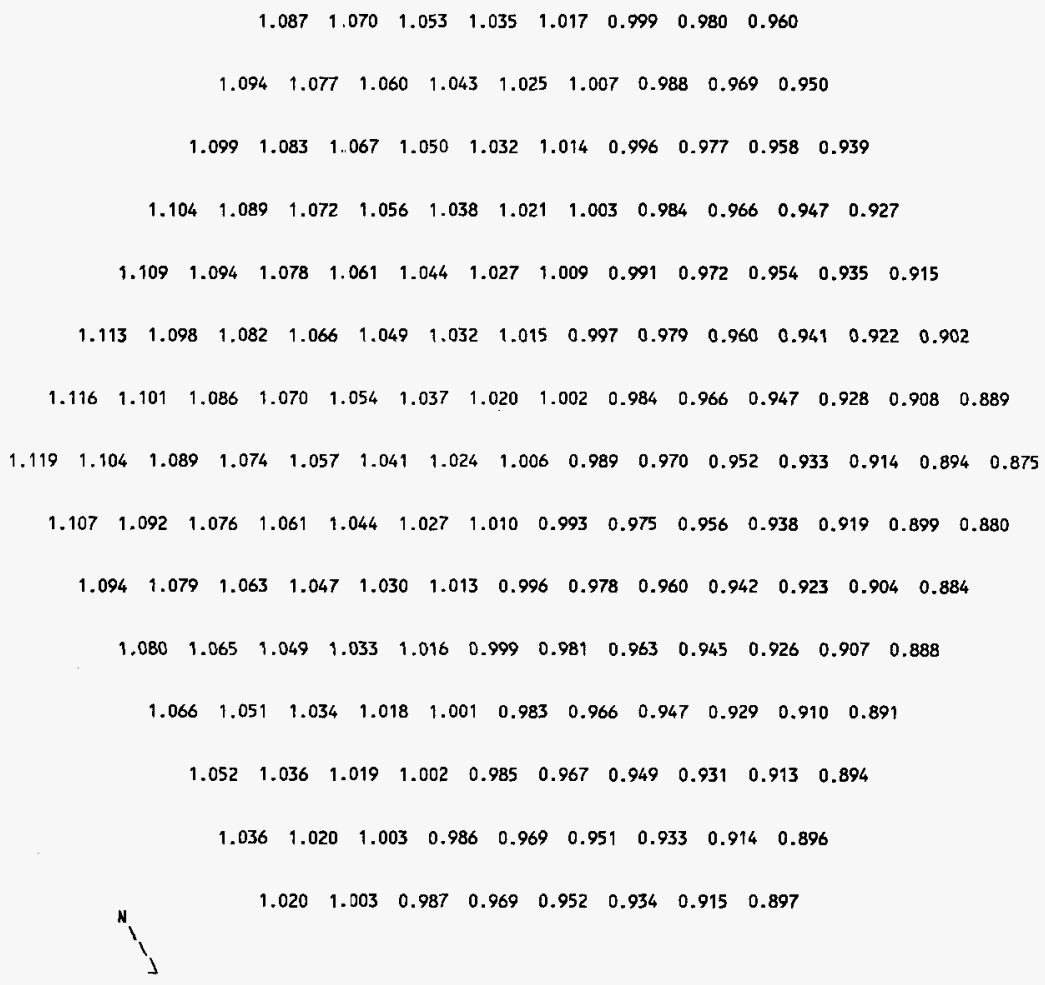


Table E1OA-1.9. MFA-1 and MFA-2 Duct Wall Flux Greater Than 0.1 MeV at EOC 10A-1

$\begin{array}{ccc}\text { SIDE } & \begin{array}{c}\text { FLUX }>0.1 \\ \text { MFA-1 }\end{array} & \begin{array}{c}\text { MEV } \\ \left(\mathrm{n} / \mathrm{cm}^{2}-\mathrm{sec}\right) \\ \text { MFA-2 }\end{array} \\ \text { E } & 1.9763 \mathrm{E}+15 & 1.9693 \mathrm{E}+15 \\ \text { SE } & 1.7725 \mathrm{E}+15 & 2.0264 \mathrm{E}+15 \\ \text { SW } & 1.7004 \mathrm{E}+15 & 1.8524 \mathrm{E}+15 \\ \text { W } & 1.8632 \mathrm{E}+15 & 1.6275 \mathrm{E}+15 \\ \text { NW } & 2.0730 \mathrm{E}+15 & 1.5572 \mathrm{E}+15 \\ \text { NE } & 2.1323 \mathrm{E}+15 & 1.7056 \mathrm{E}+15\end{array}$

Table E10A-1.10. Assembly Outlet Temperatures and Flow Rates at EOC 10A-1

$\begin{array}{lcl} & \text { CALCULATED } \\ \text { CORE } & \text { OUTLET TEMP. } & \text { FLOW RATE } \\ \text { POS. } & \text { (DEG F) } & \text { (LEj/H) } \\ 1506 & 1077 & 116330 \\ 1404 & 922 & 228160 \\ 1405 & 894 & 210640 \\ 1505 & 884 & 210640 \\ 1507 & 868 & 210640 \\ 1606 & 918 & 172740 \\ 1607 & 880 & 172740 \\ 2507 & 1038 & 116330 \\ 2405 & 907 & 194.730 \\ 2506 & 872 & 2001300 \\ 2508 & 895 & 210640 \\ 2607 & 890 & 165660 \\ 2608 & 1038 & 85760 \\ 2609 & 875 & 175430\end{array}$


Table E1OA-1.17. Composition of MFA-1 at EOC 10A-1

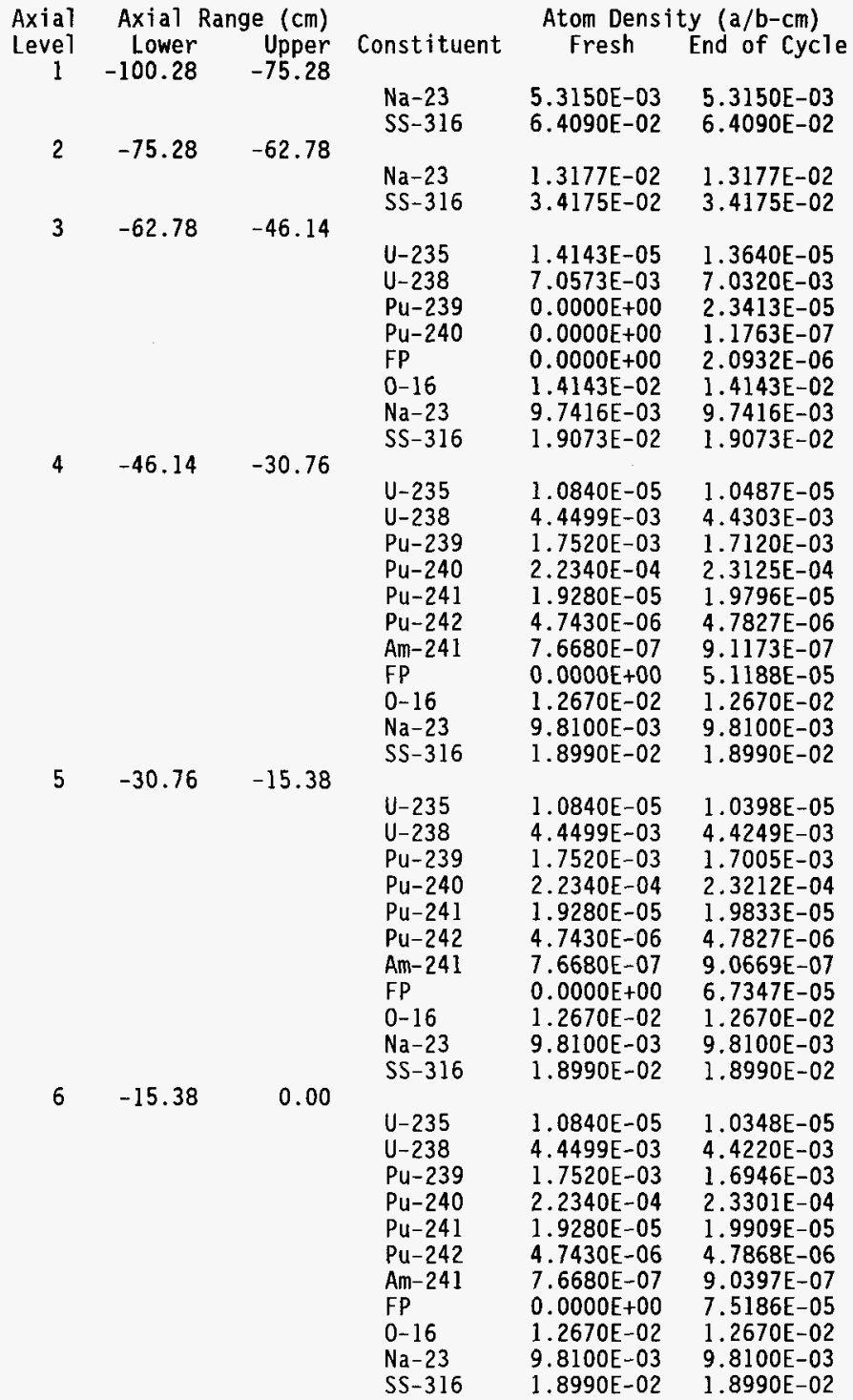




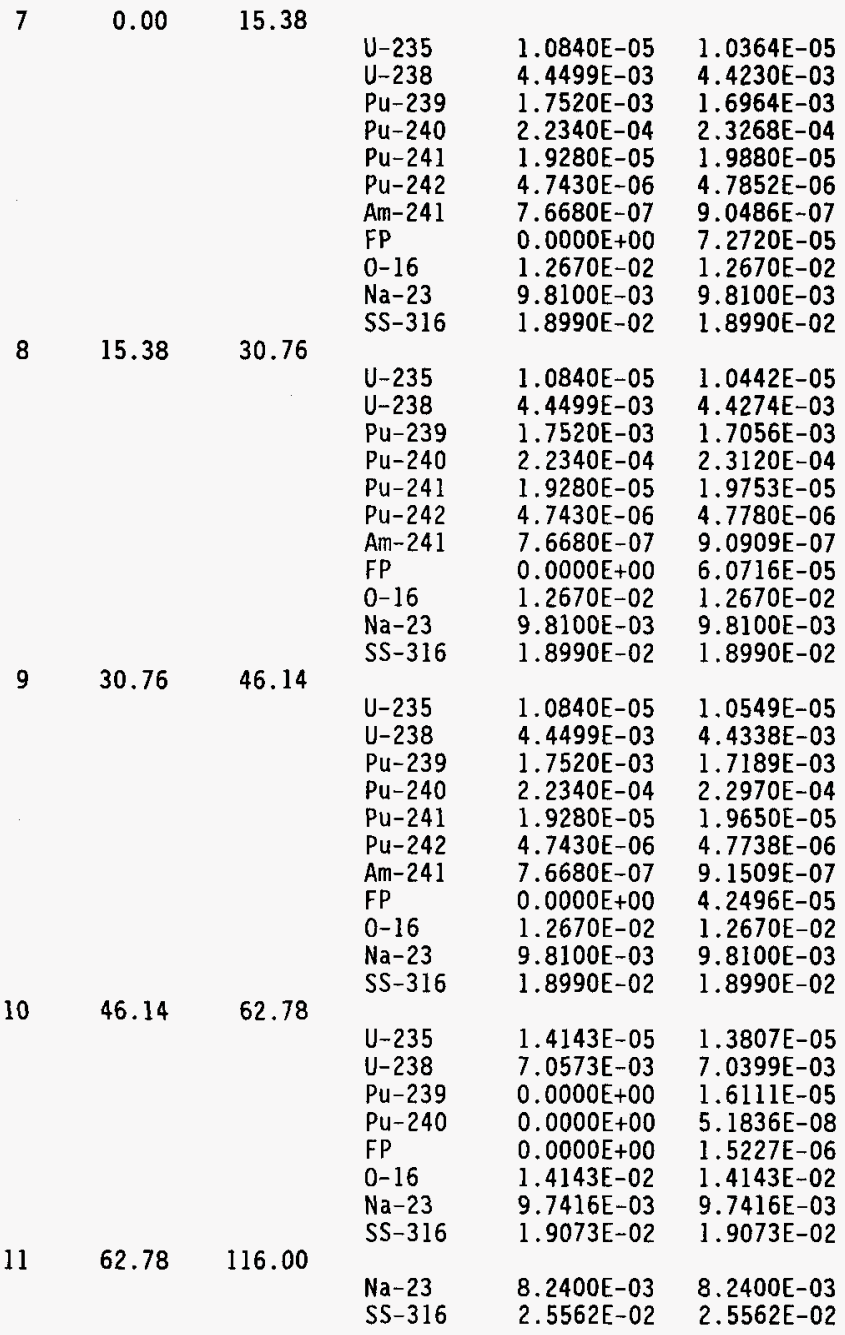


Table E1OA-1.18. Composition of MFA-2 at EOC 10A-1

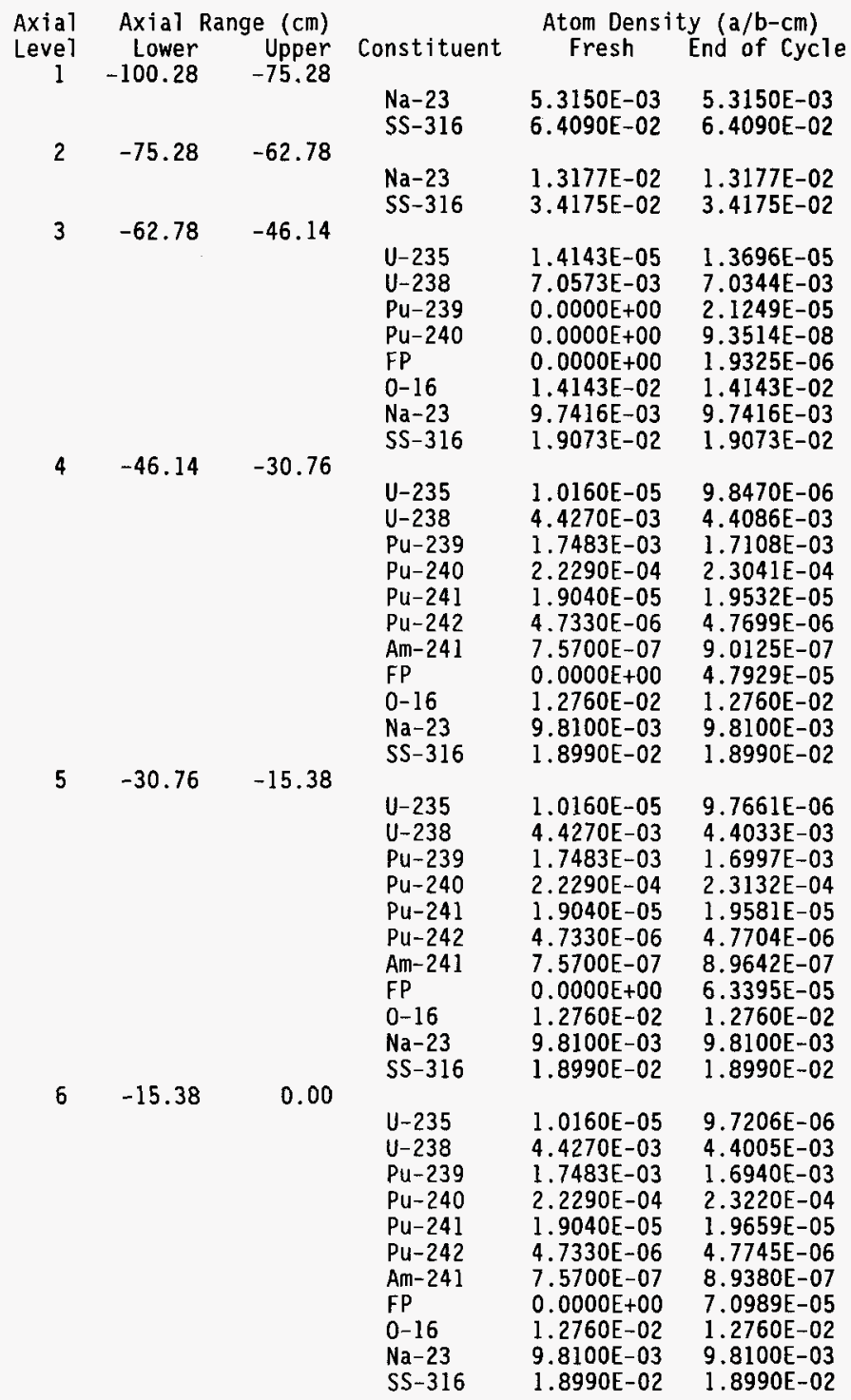


WHC-SD-FF-ANAL-009 REV 0

$\begin{array}{lll}7 & 0.00 & 15.38\end{array}$

$\begin{array}{lll}\text { U-235 } & 1.0160 \mathrm{E}-05 & 9.7343 \mathrm{E}-06 \\ \mathrm{U}-238 & 4.4270 \mathrm{E}-03 & 4.4014 \mathrm{E}-03 \\ \mathrm{PU}-239 & 1.7483 \mathrm{E}-03 & 1.6956 \mathrm{E}-03 \\ \mathrm{Pu}-240 & 2.2290 \mathrm{E}-04 & 2.3190 \mathrm{E}-04 \\ \mathrm{Pu}-241 & 1.9040 \mathrm{E}-05 & 1.9632 \mathrm{E}-05 \\ \mathrm{Pu}-242 & 4.7330 \mathrm{E}-05 & 4.7730 \mathrm{E}-05 \\ \mathrm{Am}-241 & 7.5700 \mathrm{E}-07 & 8.9458 \mathrm{E}-07 \\ \mathrm{FP} & 0.0000 \mathrm{E}+00 & 6.8798 \mathrm{E}-05 \\ 0-16 & 1.2760 \mathrm{E}-02 & 1.2760 \mathrm{E}-02 \\ \mathrm{Na}-23 & 9.8100 \mathrm{E}-03 & 9.8100 \mathrm{E}-03 \\ \mathrm{SS}-316 & 1.8990 \mathrm{E}-02 & 1.8990 \mathrm{E}-02\end{array}$

$\begin{array}{lll}8 & 15.38 & 30.76\end{array}$

$\begin{array}{lll}\mathrm{U}-235 & 1.0160 \mathrm{E}-05 & 9.8018 \mathrm{E}-06 \\ \mathrm{U}-238 & 4.4270 \mathrm{E}-03 & 4.4055 \mathrm{E}-03 \\ \mathrm{Pu}-239 & 1.7483 \mathrm{E}-03 & 1.7041 \mathrm{E}-03 \\ \mathrm{Pu}-240 & 2.2290 \mathrm{E}-04 & 2.3053 \mathrm{E}-04 \\ \mathrm{Pu}-241 & 1.9040 \mathrm{E}-05 & 1.9513 \mathrm{E}-05 \\ \mathrm{Pu}-242 & 4.7330 \mathrm{E}-06 & 4.7666 \mathrm{E}-06 \\ \mathrm{Am}-241 & 7.5700 \mathrm{E}-07 & 8.9845 \mathrm{E}-07 \\ \mathrm{FP} & 0.0000 \mathrm{E}+00 & 5.7703 \mathrm{E}-05 \\ \mathrm{O}-16 & 1.2760 \mathrm{E}-02 & 1.2760 \mathrm{E}-02 \\ \mathrm{Na}-23 & 9.8100 \mathrm{E}-03 & 9.8100 \mathrm{E}-03 \\ \mathrm{SS}-316 & 1.8990 \mathrm{E}-02 & 1.8990 \mathrm{E}-02\end{array}$

$9 \quad 30.76 \quad 46.14$

$\begin{array}{lll}\mathrm{U}-235 & 1.0160 \mathrm{E}-05 & 9.8972 \mathrm{E}-06 \\ \mathrm{U}-238 & 4.4270 \mathrm{E}-03 & 4.4115 \mathrm{E}-03 \\ \mathrm{Pu}-239 & 1.7483 \mathrm{E}-03 & 1.7167 \mathrm{E}-03 \\ \mathrm{Pu}-240 & 2.2290 \mathrm{E}-04 & 2.2909 \mathrm{E}-04 \\ \mathrm{Pu}-241 & 1.9040 \mathrm{E}-05 & 1.9409 \mathrm{E}-05 \\ \mathrm{Pu}-242 & 4.7330 \mathrm{E}-06 & 4.7626 \mathrm{E}-06 \\ \mathrm{Am}-241 & 7.5700 \mathrm{E}-07 & 9.0406 \mathrm{E}-07 \\ \mathrm{FP} & 0.0000 \mathrm{E}+00 & 4.0541 \mathrm{E}-05 \\ \mathrm{O}-16 & 1.2760 \mathrm{E}-02 & 1.2760 \mathrm{E}-02 \\ \mathrm{Na}-23 & 9.8100 \mathrm{E}-03 & 9.8100 \mathrm{E}-03 \\ \mathrm{SS}-316 & 1.8990 \mathrm{E}-02 & 1.8990 \mathrm{E}-02\end{array}$

$\begin{array}{lll}10 & 46.14 & 62.78\end{array}$

U-235 $1.4143 \mathrm{E}-05 \quad 1.3827 \mathrm{E}-05$

U-238 7.0573E-03 7.0406E-03

$\mathrm{Pu}-239 \quad 0.0000 \mathrm{E}+00 \quad 1.5406 \mathrm{E}-05$

$\mathrm{Pu}-240 \quad 0.0000 \mathrm{E}+00 \quad 4.6476 \mathrm{E}-08$

FP $\quad 0.0000 \mathrm{E}+00 \quad 1.4617 \mathrm{E}-06$

0-16 $1.4143 \mathrm{E}-02 \quad 1.4143 \mathrm{E}-02$

$\mathrm{Na}-23 \quad 9.7416 \mathrm{E}-03 \quad 9.7416 \mathrm{E}-03$

SS-316 $1.9073 \mathrm{E}-02 \quad 1.9073 \mathrm{E}-02$

$\begin{array}{llllll}11 & 62.78 & 116.00 \quad \mathrm{Na}-23 & 8.2400 \mathrm{E}-03 & 8.2400 \mathrm{E}-03\end{array}$

SS-316 2.5562E-02 2.5562E-02 
Table B10A-2.1. Fission Power Generated in MFA-1, MFA-2 and Neighboring Assemblies at BOC 10A-2

\begin{tabular}{lcccc} 
CORE & $* * * * * *$ & \multicolumn{2}{c}{ POWER IN MEGAWATTS } & $* * * * * *$ \\
POS. & BELOW CORE & IN CORE & ABOVE CORE & TOTAL PWR \\
1506 & $2.734 \mathrm{E}-02$ & $3.888 \mathrm{E}+00$ & $1.919 \mathrm{E}-02$ & $3.935 \mathrm{E}+00$ \\
1404 & $8.085 \mathrm{E}-02$ & $4.652 \mathrm{E}+00$ & $4.446 \mathrm{E}-02$ & $4.778 \mathrm{E}+00$ \\
1405 & $9.264 \mathrm{E}-03$ & $3.890 \mathrm{E}+00$ & $6.952 \mathrm{E}-03$ & $3.906 \mathrm{E}+00$ \\
1505 & $1.106 \mathrm{E}-02$ & $3.550 \mathrm{E}+00$ & $6.539 \mathrm{E}-03$ & $3.567 \mathrm{E}+00$ \\
1507 & $9.617 \mathrm{E}-03$ & $3.291 \mathrm{E}+00$ & $7.200 \mathrm{E}-03$ & $3.308 \mathrm{E}+00$ \\
1606 & $6.006 \mathrm{E}-03$ & $3.453 \mathrm{E}+00$ & $4.422 \mathrm{E}-03$ & $3.464 \mathrm{E}+00$ \\
1607 & $8.993 \mathrm{E}-03$ & $2.851 \mathrm{E}+00$ & $6.503 \mathrm{E}-03$ & $2.867 \mathrm{E}+00$ \\
2507 & $2.827 \mathrm{E}-02$ & $4.180 \mathrm{E}+00$ & $2.107 \mathrm{E}-02$ & $4.229 \mathrm{E}+00$ \\
2405 & $7.001 \mathrm{E}-02$ & $4.460 \mathrm{E}+00$ & $4.071 \mathrm{E}-02$ & $4.571 \mathrm{E}+00$ \\
2506 & $1.122 \mathrm{E}-02$ & $3.876 \mathrm{E}+00$ & $7.938 \mathrm{E}-03$ & $3.895 \mathrm{E}+00$ \\
2508 & $1.083 \mathrm{E}-02$ & $4.596 \mathrm{E}+00$ & $7.977 \mathrm{E}-03$ & $4.615 \mathrm{E}+00$ \\
2607 & $7.671 \mathrm{E}-03$ & $3.560 \mathrm{E}+00$ & $5.748 \mathrm{E}-03$ & $3.574 \mathrm{E}+00$ \\
2608 & $4.839 \mathrm{E}-03$ & $3.421 \mathrm{E}+00$ & $4.041 \mathrm{E}-03$ & $3.430 \mathrm{E}+00$ \\
2609 & $9.386 \mathrm{E}-02$ & $3.167 \mathrm{E}+00$ & $5.186 \mathrm{E}-02$ & $3.313 \mathrm{E}+00$
\end{tabular}

Table B10A-2.2. Assembly Averaged Total Flux for MFA-1 and MFA-2 at BOC 10A-2

$\begin{array}{ccc} & \text { Core } & \text { Total Flux } \\ \text { Assembly } & \text { Pos. } & \left(\mathrm{n} / \mathrm{cm}^{2}-\mathrm{sec}\right) \\ \text { MFA-1 } & 1506 & 2.855 \mathrm{E}+15 \\ \text { MFA-2 } & 2507 & 3.063 \mathrm{E}+15\end{array}$


Table B1OA-2.3. Axial Distribution of Total Flux, Fast Flux and Power in MFA-1 at BOC $10 \mathrm{~A}-2$

$\begin{array}{rccc}z(\mathrm{~cm}) & \text { Tota Flux } & \text { F1ux }>0.1 \mathrm{MeV} & \text { Power } \\ -97.78 & 2.7490 \mathrm{E}-02 & 1.3904 \mathrm{E}-02 & 0.0000 \mathrm{E}+00 \\ -92.78 & 5.9094 \mathrm{E}-02 & 2.6852 \mathrm{E}-02 & 0.0000 \mathrm{E}+00 \\ -87.78 & 9.4762 \mathrm{E}-02 & 4.2972 \mathrm{E}-02 & 0.0000 \mathrm{E}+00 \\ -82.78 & 1.3719 \mathrm{E}-01 & 6.4106 \mathrm{E}-02 & 0.0000 \mathrm{E}+00 \\ -77.78 & 1.8909 \mathrm{E}-01 & 9.2523 \mathrm{E}-02 & 0.0000 \mathrm{E}+00 \\ -72.15 & 2.4780 \mathrm{E}-01 & 1.2927 \mathrm{E}-01 & 0.0000 \mathrm{E}+00 \\ -65.90 & 3.0612 \mathrm{E}-01 & 1.7541 \mathrm{E}-01 & 0.0000 \mathrm{E}+00 \\ -60.35 & 3.6758 \mathrm{E}-01 & 2.3738 \mathrm{E}-01 & 2.5138 \mathrm{E}-02 \\ -55.49 & 4.4930 \mathrm{E}-01 & 3.2169 \mathrm{E}-01 & 3.3599 \mathrm{E}-02 \\ -50.62 & 5.4496 \mathrm{E}-01 & 4.3254 \mathrm{E}-01 & 4.7534 \mathrm{E}-02 \\ -47.17 & 6.2244 \mathrm{E}-01 & 5.3821 \mathrm{E}-01 & 6.4395 \mathrm{E}-02 \\ -43.58 & 7.0413 \mathrm{E}-01 & 6.5858 \mathrm{E}-01 & 7.4425 \mathrm{E}-01 \\ -38.45 & 8.2126 \mathrm{E}-01 & 8.0299 \mathrm{E}-01 & 8.3479 \mathrm{E}-01 \\ -33.32 & 9.2956 \mathrm{E}-01 & 9.2555 \mathrm{E}-01 & 9.3464 \mathrm{E}-01 \\ -28.20 & 1.0240 \mathrm{E}+00 & 1.0273 \mathrm{E}+00 & 1.0197 \mathrm{E}+00 \\ -23.07 & 1.1025 \mathrm{E}+00 & 1.1098 \mathrm{E}+00 & 1.0962 \mathrm{E}+00 \\ -17.94 & 1.1636 \mathrm{E}+00 & 1.1729 \mathrm{E}+00 & 1.1560 \mathrm{E}+00 \\ -12.82 & 1.2063 \mathrm{E}+00 & 1.2163 \mathrm{E}+00 & 1.1944 \mathrm{E}+00 \\ -7.69 & 1.2306 \mathrm{E}+00 & 1.2409 \mathrm{E}+00 & 1.2181 \mathrm{E}+00 \\ -2.56 & 1.2360 \mathrm{E}+00 & 1.2466 \mathrm{E}+00 & 1.2233 \mathrm{E}+00 \\ 2.56 & 1.2227 \mathrm{E}+00 & 1.2335 \mathrm{E}+00 & 1.2111 \mathrm{E}+00 \\ 7.69 & 1.1909 \mathrm{E}+00 & 1.2018 \mathrm{E}+00 & 1.1796 \mathrm{E}+00 \\ 12.82 & 1.1415 \mathrm{E}+00 & 1.1523 \mathrm{E}+00 & 1.1307 \mathrm{E}+00 \\ 17.94 & 1.0758 \mathrm{E}+00 & 1.0864 \mathrm{E}+00 & 1.0707 \mathrm{E}+00 \\ 23.07 & 9.9519 \mathrm{E}-01 & 1.0044 \mathrm{E}+00 & 9.9089 \mathrm{E}-01 \\ 28.20 & 9.0174 \mathrm{E}-01 & 9.0781 \mathrm{E}-01 & 8.9891 \mathrm{E}-01 \\ 33.32 & 7.9785 \mathrm{E}-01 & 7.9824 \mathrm{E}-01 & 8.0306 \mathrm{E}-01 \\ 38.45 & 6.8590 \mathrm{E}-01 & 6.7530 \mathrm{E}-01 & 6.9632 \mathrm{E}-01 \\ 43.58 & 5.7029 \mathrm{E}-01 & 5.3923 \mathrm{E}-01 & 5.9727 \mathrm{E}-01 \\ 47.17 & 4.9150 \mathrm{E}-01 & 4.3247 \mathrm{E}-01 & 4.9498 \mathrm{E}-02 \\ 50.62 & 4.1725 \mathrm{E}-01 & 3.3897 \mathrm{E}-01 & 3.4897 \mathrm{E}-02 \\ 55.49 & 3.2587 \mathrm{E}-01 & 2.4130 \mathrm{E}-01 & 2.2711 \mathrm{E}-02 \\ 60.35 & 2.4701 \mathrm{E}-01 & 1.6701 \mathrm{E}-01 & 1.5153 \mathrm{E}-02 \\ 64.98 & 1.9879 \mathrm{E}-01 & 1.2488 \mathrm{E}-01 & 0.0000 \mathrm{E}+00 \\ 69.39 & 1.7069 \mathrm{E}-01 & 1.0281 \mathrm{E}-01 & 0.0000 \mathrm{E}+00 \\ 76.46 & 1.3050 \mathrm{E}-01 & 7.3220 \mathrm{E}-02 & 0.0000 \mathrm{E}+00 \\ 86.80 & 8.5638 \mathrm{E}-02 & 4.4335 \mathrm{E}-02 & 0.0000 \mathrm{E}+00 \\ 98.14 & 4.9635 \mathrm{E}-02 & 2.4314 \mathrm{E}-02 & 0.0000 \mathrm{E}+00 \\ 110.00 & 2.1333 \mathrm{E}-02 & 1.0931 \mathrm{E}-02 & 0.0000 \mathrm{E}+00 \\ & & & \end{array}$


Table BIOA-2.4. Axial Distribution of Total Flux, Fast Flux and Power in MFA-2 at BOC $10 \mathrm{~A}-2$

$\begin{array}{rccc}z(\mathrm{~cm}) & \text { Total Flux } & \text { Flux }>0.1 \mathrm{MeV} & \text { Power } \\ -97.78 & 2.5287 \mathrm{E}-02 & 1.2895 \mathrm{E}-02 & 0.0000 \mathrm{E}+00 \\ -92.78 & 5.4304 \mathrm{E}-02 & 2.4889 \mathrm{E}-02 & 0.0000 \mathrm{E}+00 \\ -87.78 & 8.7078 \mathrm{E}-02 & 3.9832 \mathrm{E}-02 & 0.0000 \mathrm{E}+00 \\ -82.78 & 1.2618 \mathrm{E}-01 & 5.9474 \mathrm{E}-02 & 0.0000 \mathrm{E}+00 \\ -77.78 & 1.7434 \mathrm{E}-01 & 8.6027 \mathrm{E}-02 & 0.0000 \mathrm{E}+00 \\ -72.15 & 2.2951 \mathrm{E}-01 & 1.2067 \mathrm{E}-01 & 0.0000 \mathrm{E}+00 \\ -65.90 & 2.8617 \mathrm{E}-01 & 1.6524 \mathrm{E}-01 & 0.0000 \mathrm{E}+00 \\ -60.35 & 3.4757 \mathrm{E}-01 & 2.2611 \mathrm{E}-01 & 2.3606 \mathrm{E}-02 \\ -55.49 & 4.2917 \mathrm{E}-01 & 3.0902 \mathrm{E}-01 & 3.2126 \mathrm{E}-02 \\ -50.62 & 5.2569 \mathrm{E}-01 & 4.1864 \mathrm{E}-01 & 4.6080 \mathrm{E}-02 \\ -47.17 & 6.0460 \mathrm{E}-01 & 5.2362 \mathrm{E}-01 & 6.2868 \mathrm{E}-02 \\ -43.58 & 6.8800 \mathrm{E}-01 & 6.4360 \mathrm{E}-01 & 7.2608 \mathrm{E}-01 \\ -38.45 & 8.0749 \mathrm{E}-01 & 7.8929 \mathrm{E}-01 & 8.2053 \mathrm{E}-01 \\ -33.32 & 9.1807 \mathrm{E}-01 & 9.1397 \mathrm{E}-01 & 9.2286 \mathrm{E}-01 \\ -28.20 & 1.0148 \mathrm{E}+00 & 1.0182 \mathrm{E}+00 & 1.0104 \mathrm{E}+00 \\ -23.07 & 1.0957 \mathrm{E}+00 & 1.1033 \mathrm{E}+00 & 1.0893 \mathrm{E}+00 \\ -17.94 & 1.1595 \mathrm{E}+00 & 1.1691 \mathrm{E}+00 & 1.1518 \mathrm{E}+00 \\ -12.82 & 1.2048 \mathrm{E}+00 & 1.2151 \mathrm{E}+00 & 1.1930 \mathrm{E}+00 \\ -7.69 & 1.2311 \mathrm{E}+00 & 1.2418 \mathrm{E}+00 & 1.2188 \mathrm{E}+00 \\ -2.56 & 1.2382 \mathrm{E}+00 & 1.2491 \mathrm{E}+00 & 1.2257 \mathrm{E}+00 \\ 2.56 & 1.2263 \mathrm{E}+00 & 1.2374 \mathrm{E}+00 & 1.2147 \mathrm{E}+00 \\ 7.69 & 1.1958 \mathrm{E}+00 & 1.2070 \mathrm{E}+00 & 1.1846 \mathrm{E}+00 \\ 12.82 & 1.1478 \mathrm{E}+00 & 1.1588 \mathrm{E}+00 & 1.1373 \mathrm{E}+00 \\ 17.94 & 1.0836 \mathrm{E}+00 & 1.0941 \mathrm{E}+00 & 1.0786 \mathrm{E}+00 \\ 23.07 & 1.0040 \mathrm{E}+00 & 1.0128 \mathrm{E}+00 & 1.0000 \mathrm{E}+00 \\ 28.20 & 9.1073 \mathrm{E}-01 & 9.1632 \mathrm{E}-01 & 9.0834 \mathrm{E}-01 \\ 33.32 & 8.0627 \mathrm{E}-01 & 8.0591 \mathrm{E}-01 & 8.1171 \mathrm{E}-01 \\ 38.45 & 6.9279 \mathrm{E}-01 & 6.8129 \mathrm{E}-01 & 7.0360 \mathrm{E}-01 \\ 43.58 & 5.7507 \mathrm{E}-01 & 5.4293 \mathrm{E}-01 & 6.0278 \mathrm{E}-01 \\ 47.17 & 4.9475 \mathrm{E}-01 & 4.3465 \mathrm{E}-01 & 5.0486 \mathrm{E}-02 \\ 50.62 & 4.1915 \mathrm{E}-01 & 3.4011 \mathrm{E}-01 & 3.5631 \mathrm{E}-02 \\ 55.49 & 3.2700 \mathrm{E}-01 & 2.4186 \mathrm{E}-01 & 2.3215 \mathrm{E}-02 \\ 60.35 & 2.4813 \mathrm{E}-01 & 1.6747 \mathrm{E}-01 & 1.5505 \mathrm{E}-02 \\ 64.98 & 1.9982 \mathrm{E}-01 & 1.2503 \mathrm{E}-01 & 0.0000 \mathrm{E}+00 \\ 69.39 & 1.7116 \mathrm{E}-01 & 1.0252 \mathrm{E}-01 & 0.0000 \mathrm{E}+00 \\ 76.46 & 1.3009 \mathrm{E}-01 & 7.2401 \mathrm{E}-02 & 0.0000 \mathrm{E}+00 \\ 86.80 & 8.4762 \mathrm{E}-02 & 4.3366 \mathrm{E}-02 & 0.0000 \mathrm{E}+00 \\ 98.14 & 4.8762 . \mathrm{E}-02 & 2.3512 \mathrm{E}-02 & 0.0000 \mathrm{E}+00 \\ 110.00 & 2.0658 \mathrm{E}-02 & 1.0394 \mathrm{E}-02 & 0.0000 \mathrm{E}+00 \\ & & & \end{array}$




\section{WHC-SD-FF-ANAL-009 REV 0}

Table B10A-2.5. Fission Power Distribution by Pin in MFA-1 at BOC 10A-2

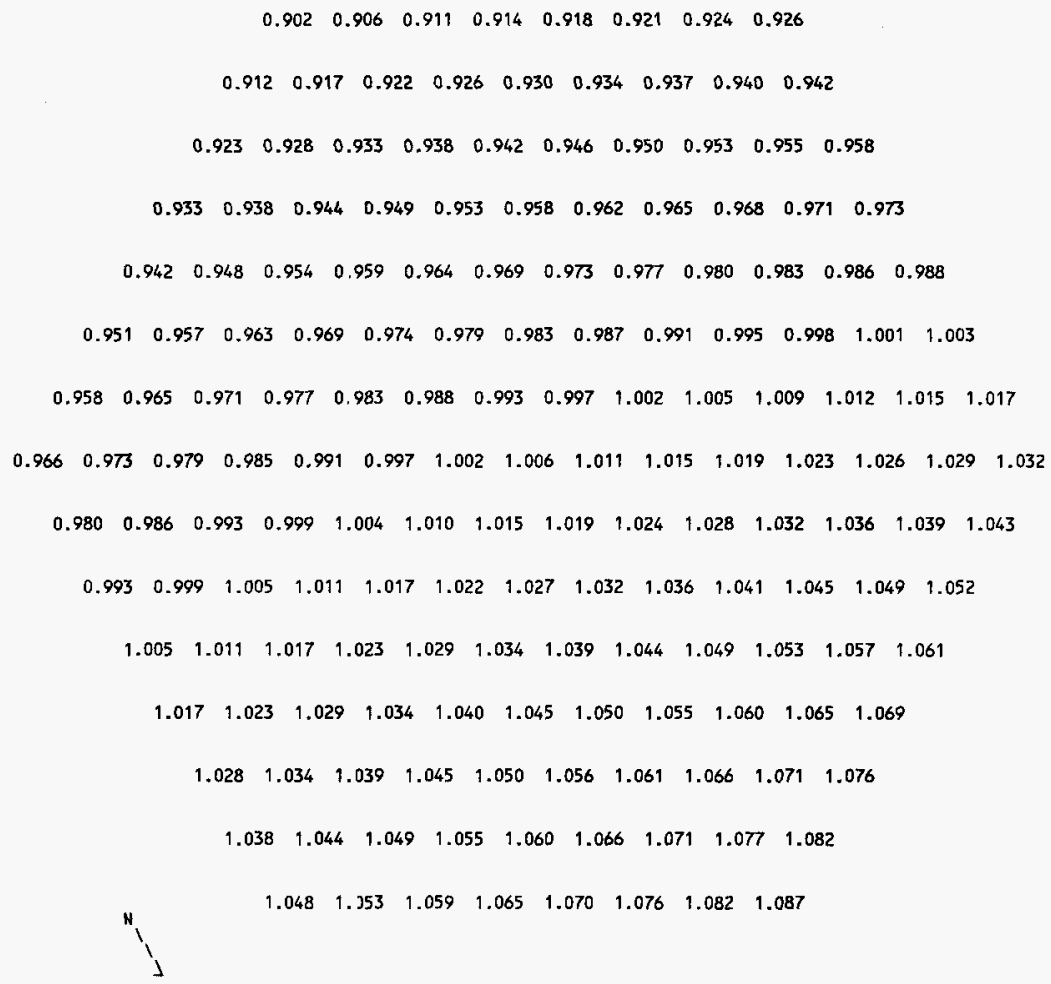




\section{WHC-SD-FF-ANAL-009 REV 0}

Tab7e B10A-2.6. Fission Power Distribution by Pin in MFA-2 at BOC 10A-2

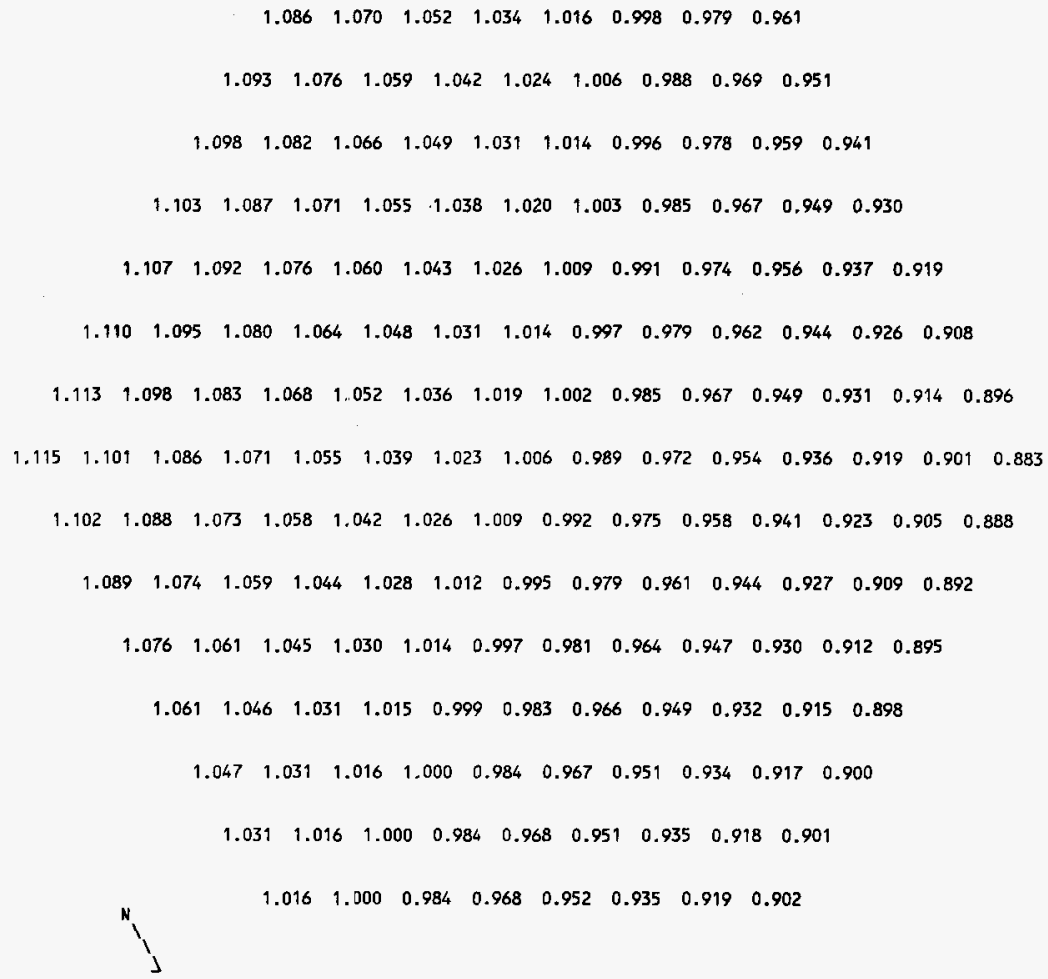


Table B10A-2.7. Total Flux Distribution by Pin in MFA-1 at BOC 10A-2

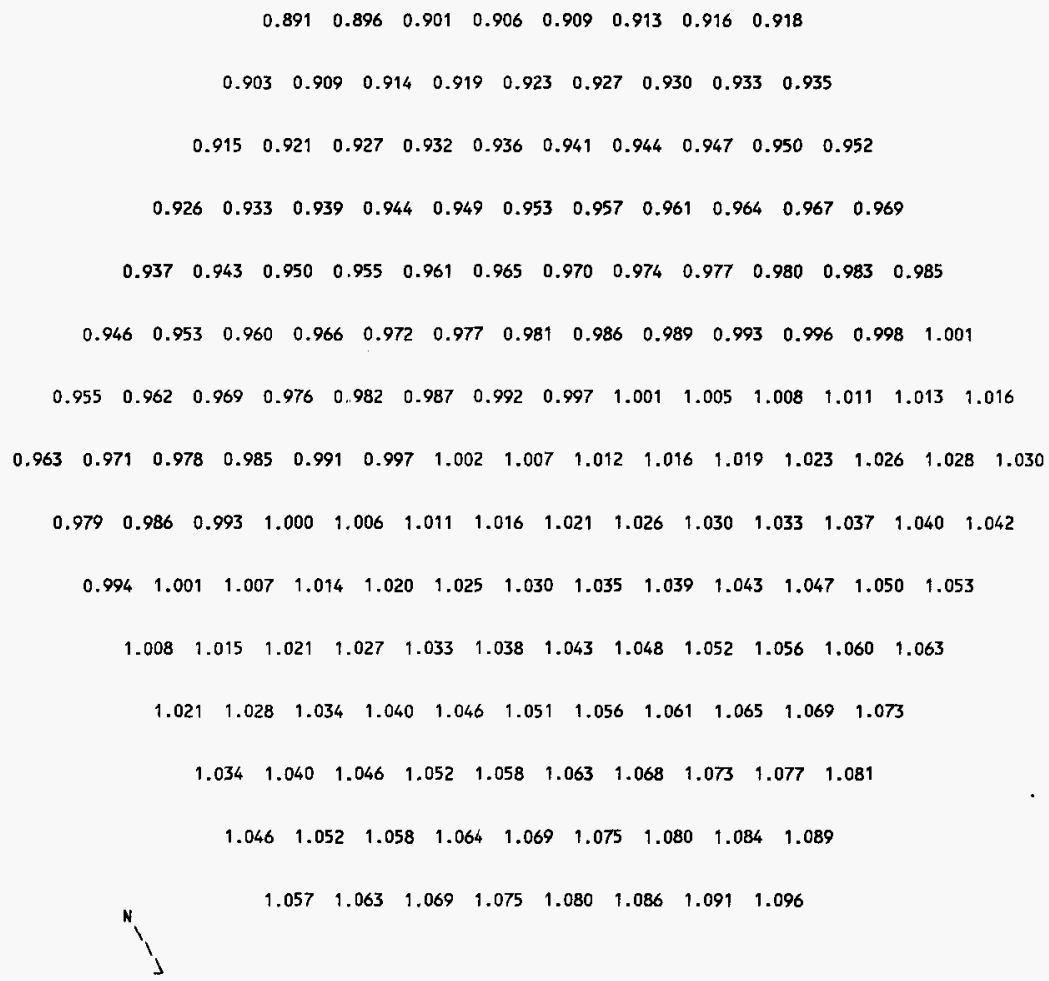


Table B10A-2.8. Total Flux Distribution by Pin in MFA-2 at BOC 10A-2

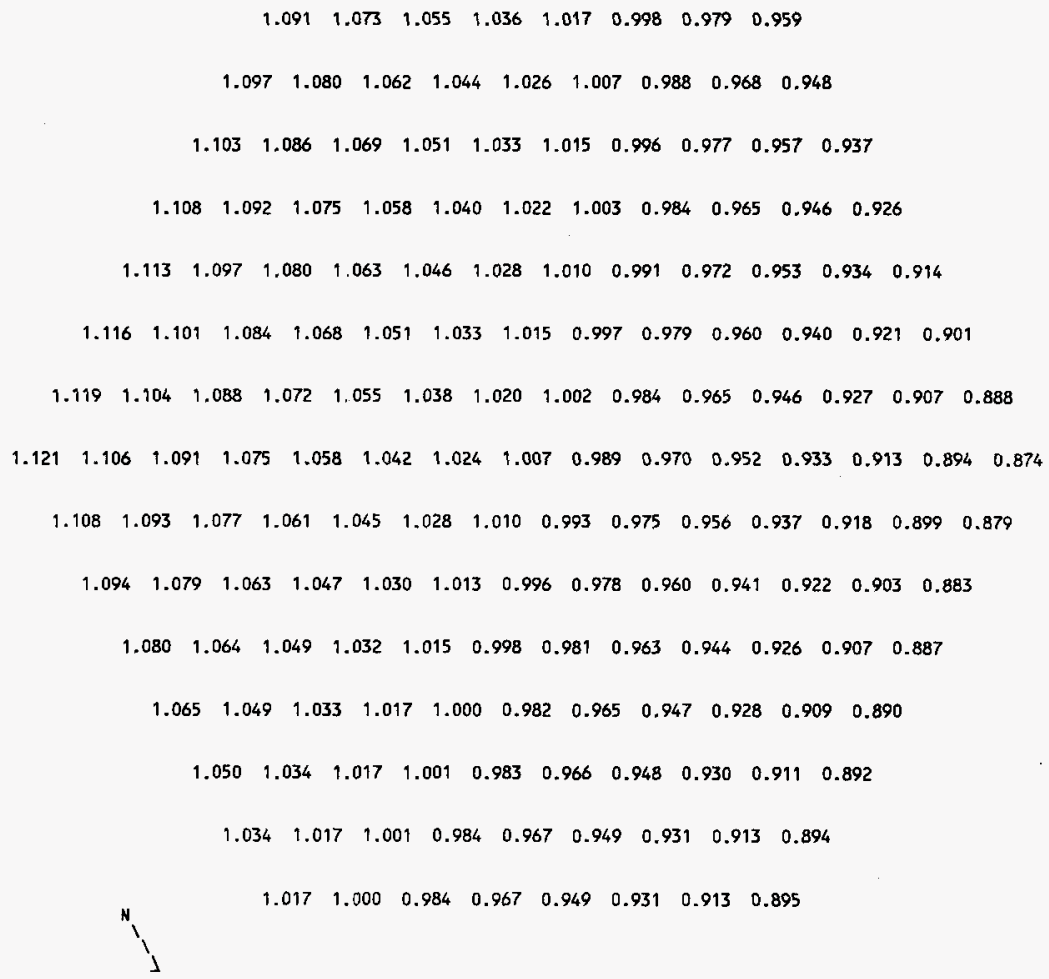


Table BlOA-2.9. MFA-1 and MFA-2 Duct Wall Flux Greater Than $0.1 \mathrm{MeV}$ at $B O C$ 10A-2

$\begin{array}{ccc}\text { SIDE } & \begin{array}{c}\text { FLUX > 0.1 } \\ \text { MFA-1 }\end{array} & \begin{array}{c}\text { MEV } \\ \left(\mathrm{n} / \mathrm{cm}^{2}-\mathrm{sec}\right. \\ \text { MFA-2 }\end{array} \\ \text { E } & 1.8656 \mathrm{E}+15 & 2.1390 \mathrm{E}+15 \\ \text { SE } & 1.6677 \mathrm{E}+15 & 2.2249 \mathrm{E}+15 \\ \text { SW } & 1.6006 \mathrm{E}+15 & 2.0170 \mathrm{E}+15 \\ \text { W } & 1.7613 \mathrm{E}+15 & 1.7603 \mathrm{E}+15 \\ \text { NW } & 1.9781 \mathrm{E}+15 & 1.61334 \mathrm{E}+15 \\ \text { NE } & 2.0318 \mathrm{E}+15 & 1.8419 \mathrm{E}+15\end{array}$

Tab1e B10A-2.10. Assembiy Outlet Temperatures and Flow Rates at BOC 10A-2

\begin{tabular}{|c|c|c|c|}
\hline $\begin{array}{l}\text { CORE } \\
\text { POS. } \\
1506 \\
1404 \\
1405 \\
1505 \\
1507 \\
1606 \\
1607 \\
2507 \\
2405 \\
2506 \\
2508 \\
2607 \\
2608 \\
2609\end{array}$ & $\begin{array}{c}\text { OUTLET T } \\
\text { MEASURED } \\
1034 \\
917 \\
891 \\
883 \\
873 \\
902 \\
871 \\
1063 \\
949 \\
902 \\
932 \\
926 \\
903 \\
898\end{array}$ & $\begin{array}{c}\text { P. (DEG F) } \\
\text { CALCULATED } \\
1039 \\
91.1 \\
887 \\
867 \\
855 \\
898 \\
864 \\
1065 \\
938 \\
894 \\
922 \\
911 \\
9010 \\
895\end{array}$ & $\begin{array}{c}\text { FLOW RATE } \\
(L B / H) \\
114940 \\
225440 \\
208130 \\
208130 \\
208130 \\
170680 \\
170680 \\
114940 \\
192410 \\
197920 \\
208130 \\
163690 \\
170680 \\
173340\end{array}$ \\
\hline
\end{tabular}




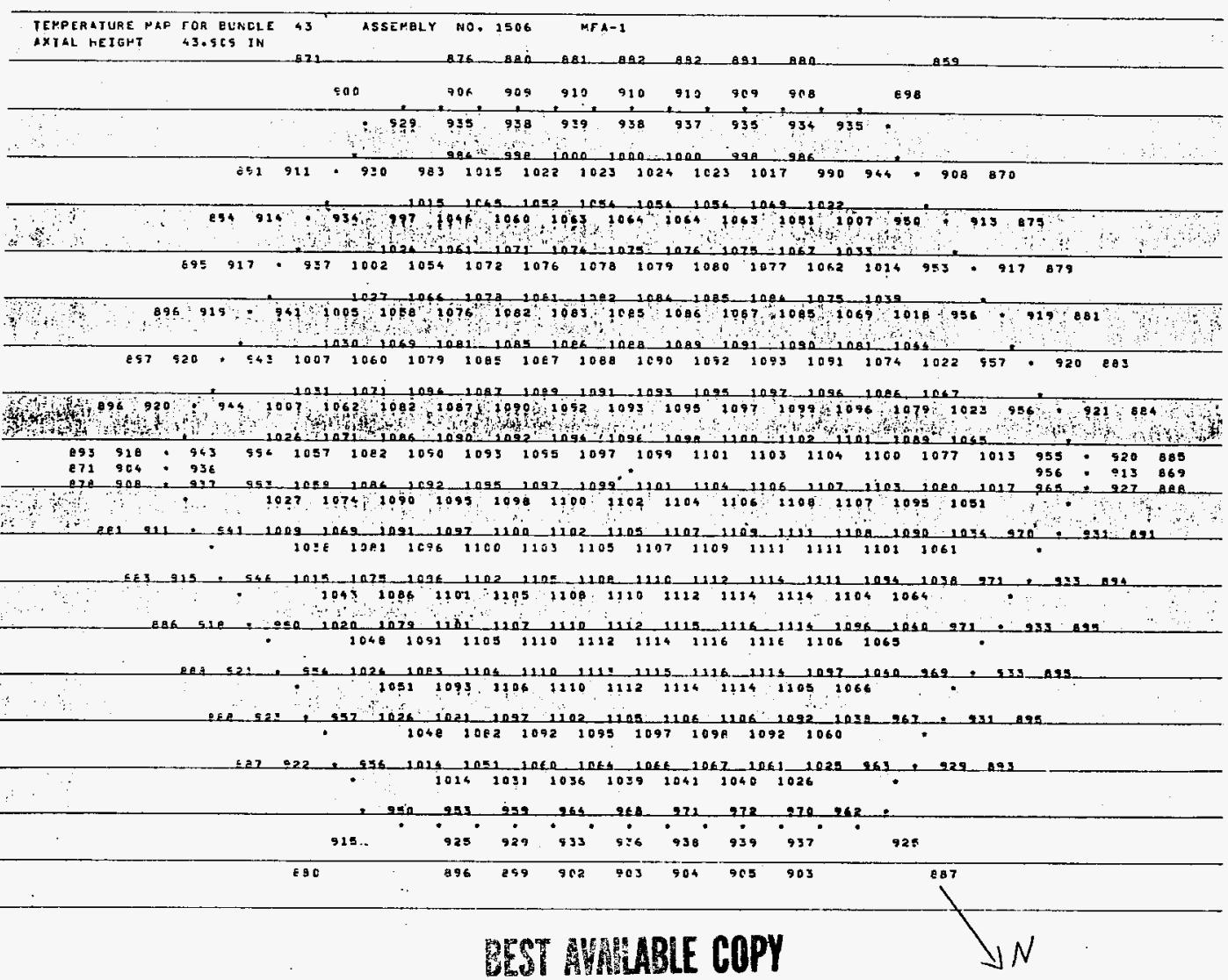


Table B10A-2.12. MFA-2 Sodium Subchannel Temperatures at Top of Core Elevation for BOC 10A-2

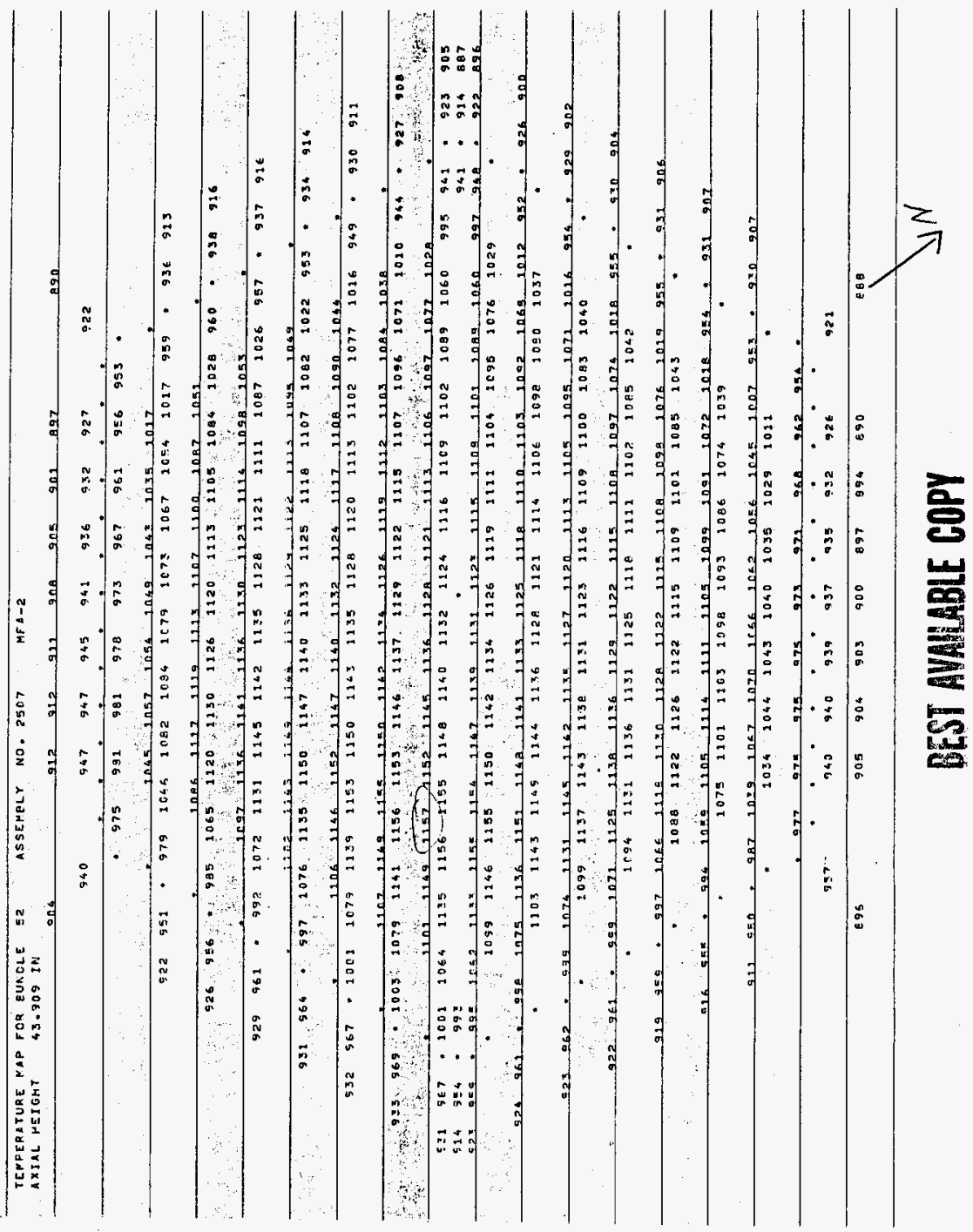


Table B10A-2.13. MFA-1 Sodium Subchannel Temperatures at Elevation of Upper Axial Blanket for BOC 10A-2

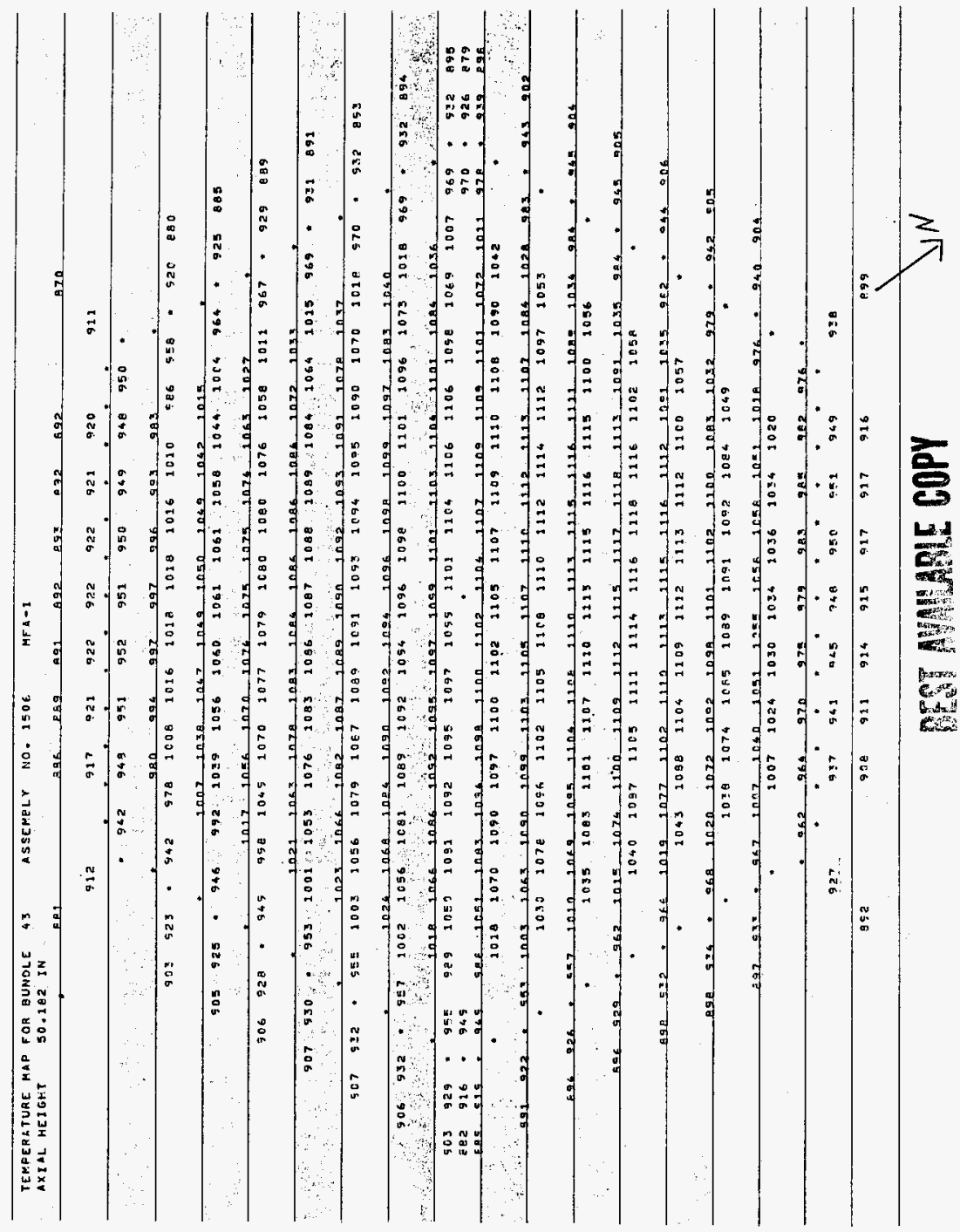


Table B10A-2.14. MFA-2 Sodium Subchanne1 Temperatures at Elevation of Upper Axial Blanket for BOC 10A-2

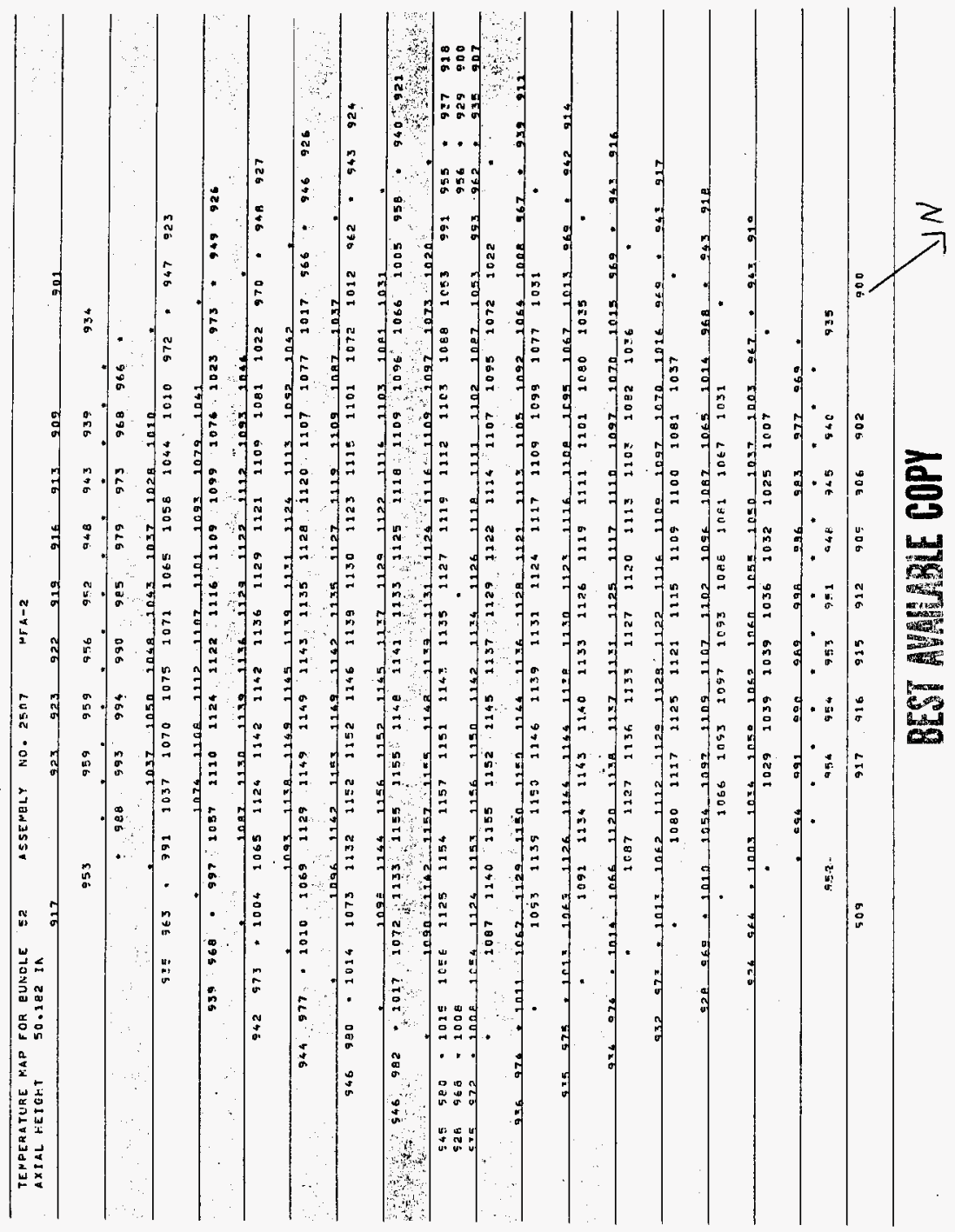


Table B10A-2.15. MFA-1 Sodium Subchannel Temperatures at Top of Fuel Pin Bundle for BOC 10A-2

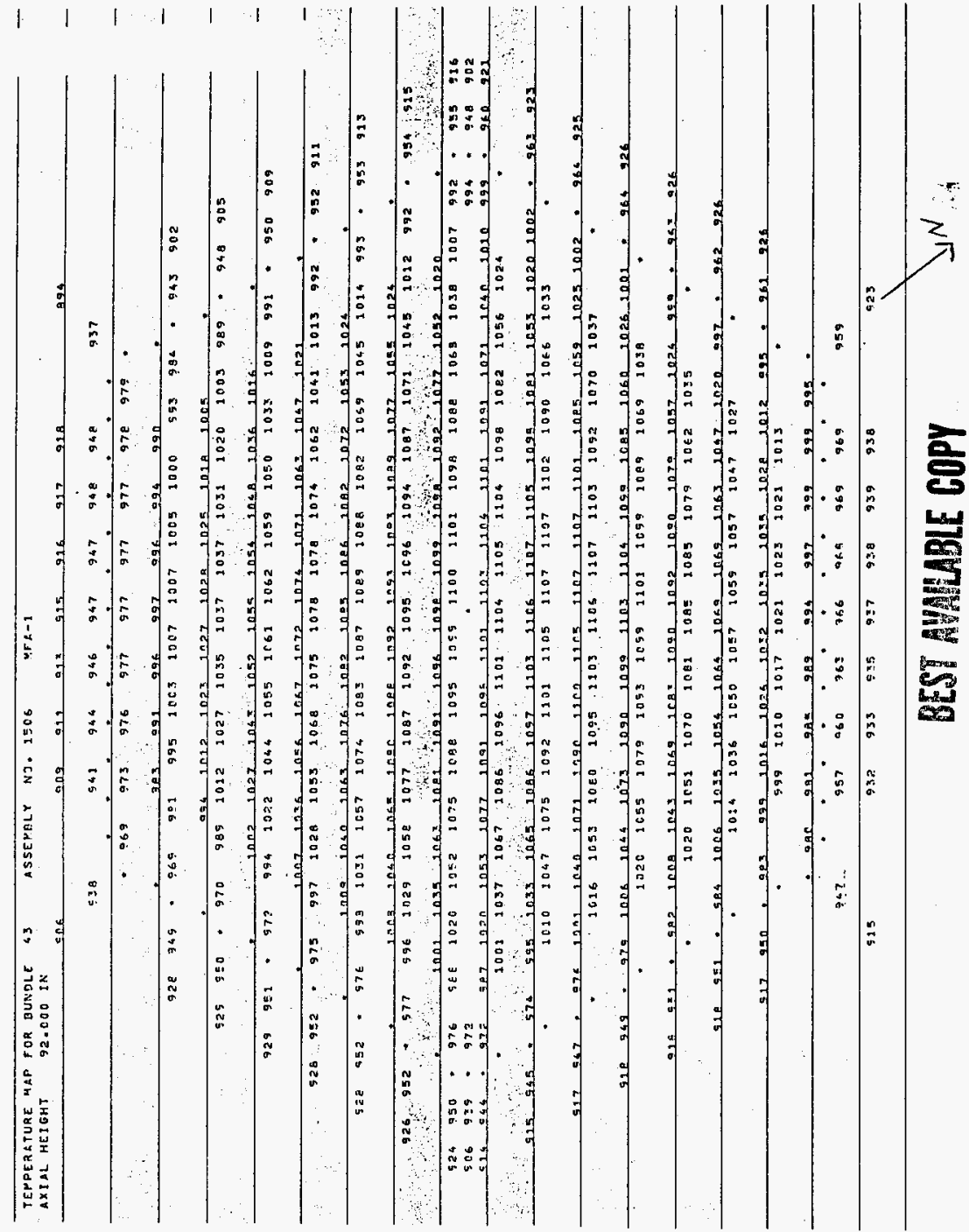


Table B10A-2.16. MFA-2 Sodium Subchannel Temperatures at Tap of Fuel Pin Bundle for BOC 10A-2

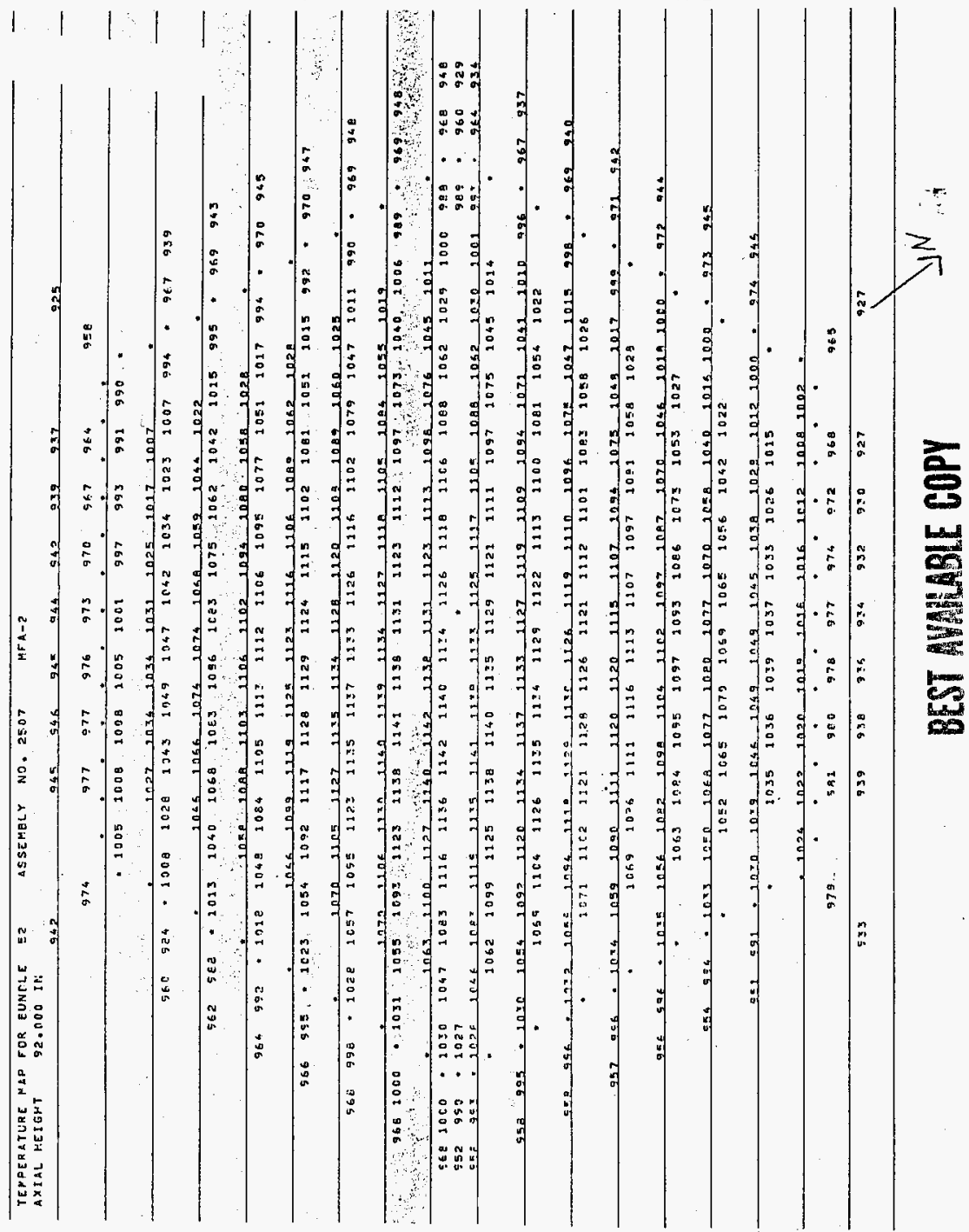


Table E10A-2.1. Fission Power Generated in MFA-1, MFA-2 and Neighboring Assemblies at EOC 10A-2

\begin{tabular}{|c|c|c|c|c|}
\hline $\begin{array}{l}\text { CORE } \\
\text { POS. } \\
1506 \\
1404 \\
1405 \\
1505 \\
1507 \\
1606 \\
1607 \\
2507 \\
2405 \\
2506\end{array}$ & $\begin{array}{l}\star \star \star \star \star \star \star * \\
\text { BELOW CORE } \\
2.921 \mathrm{E}-02 \\
8.338 \mathrm{E}-02 \\
9.579 \mathrm{E}-03 \\
1.132 \mathrm{E}-02 \\
9.834 \mathrm{E}-03 \\
6.224 \mathrm{E}-03 \\
9.178 \mathrm{E}-03 \\
3.013 \mathrm{E}-02 \\
7.222 \mathrm{E}-02 \\
1.148 \mathrm{E}-02 \\
1.112 \mathrm{E}-02 \\
7.877 \mathrm{E}-03 \\
5.014 \mathrm{E}-03 \\
9.483 \mathrm{E}-02\end{array}$ & $\begin{array}{c}\text { POWER IN } \\
\text { IN CORE } \\
3.887 E+00 \\
4.651 E+00 \\
3.889 E+00 \\
3.553 E+00 \\
3.292 E+00 \\
3.458 E+00 \\
2.857 E+00 \\
4.163 E+00 \\
4.441 E+00 \\
3.867 E+00 \\
4.576 E+00 \\
3.554 E+00 \\
3.415 E+00 \\
3.165 E+00\end{array}$ & $\begin{array}{r}\text { MEGAWATTS } \\
\text { ABOVE CORE } \\
2.016 \mathrm{E}-02 \\
4.576 \mathrm{E}-02 \\
7.163 \mathrm{E}-03 \\
6.683 \mathrm{E}-03 \\
7.360 \mathrm{E}-03 \\
4.557 \mathrm{E}-03 \\
6.641 \mathrm{E}-03 \\
2.213 \mathrm{E}-02 \\
4.198 \mathrm{E}-02 \\
8.117 \mathrm{E}-03 \\
8.175 \mathrm{E}-03 \\
5.889 \mathrm{E}-03 \\
4.168 \mathrm{E}-03 \\
5.263 \mathrm{E}-02\end{array}$ & $\begin{array}{l}* \star * \star \star \\
\text { TOTAL PWR } \\
3.936 \mathrm{E}+00 \\
4.780 \mathrm{E}+00 \\
3.905 \mathrm{E}+00 \\
3.571 \mathrm{E}+00 \\
3.310 \mathrm{E}+00 \\
3.468 \mathrm{E}+00 \\
2.872 \mathrm{E}+00 \\
4.216 \mathrm{E}+00 \\
4.555 \mathrm{E}+00 \\
3.887 \mathrm{E}+00 \\
4.595 \mathrm{E}+00 \\
3.568 \mathrm{E}+00 \\
3.424 \mathrm{E}+00 \\
3.312 \mathrm{E}+00\end{array}$ \\
\hline
\end{tabular}

Table E10A-2.2. Assembly Averaged Total Flux for MFA-1 and MFA-2 at EOC 10A-2

$\begin{array}{ccc}\text { Core } & \text { Total Flux } \\ \text { Assembly } & \text { Pos. } & \begin{array}{c}\left.\text { (n/ } / \mathrm{cm}^{2}-\mathrm{sec}\right) \\ \text { MFA-1 }\end{array} \\ \text { MFA-2 } & 2506 & 2.873 \mathrm{E}+15 \\ 3.073 \mathrm{E}+15\end{array}$


Table E10A-2.3. Axial Distribution of Total Flux, Fast Flux and Power in MFA-1 at EOC 10A-2

$\begin{array}{rccc}z(\mathrm{~cm}) & \text { Total Flux } & \text { Flux }>0.1 \mathrm{MeV} & \text { Power } \\ -97.78 & 2.7532 \mathrm{E}-02 & 1.3945 \mathrm{E}-02 & 0.0000 \mathrm{E}+00 \\ -92.78 & 5.9177 \mathrm{E}-02 & 2.6930 \mathrm{E}-02 & 0.0000 \mathrm{E}+00 \\ -87.78 & 9.4892 \mathrm{E}-02 & 4.3095 \mathrm{E}-02 & 0.0000 \mathrm{E}+00 \\ -82.78 & 1.3736 \mathrm{E}-01 & 6.4284 \mathrm{E}-02 & 0.0000 \mathrm{E}+00 \\ -77.78 & 1.8931 \mathrm{E}-01 & 9.2779 \mathrm{E}-02 & 0.0000 \mathrm{E}+00 \\ -72.15 & 2.4805 \mathrm{E}-01 & 1.2963 \mathrm{E}-01 & 0.0000 \mathrm{E}+00 \\ -65.90 & 3.0640 \mathrm{E}-01 & 1.7590 \mathrm{E}-01 & 0.0000 \mathrm{E}+00 \\ -60.35 & 3.6787 \mathrm{E}-01 & 2.3809 \mathrm{E}-01 & 2.7586 \mathrm{E}-02 \\ -55.49 & 4.4966 \mathrm{E}-01 & 3.2250 \mathrm{E}-01 & 3.6255 \mathrm{E}-02 \\ -50.62 & 5.4533 \mathrm{E}-01 & 4.3337 \mathrm{E}-01 & 5.0389 \mathrm{E}-02 \\ -47.17 & 6.2277 \mathrm{E}-01 & 5.3893 \mathrm{E}-01 & 6.7368 \mathrm{E}-02 \\ -43.58 & 7.0446 \mathrm{E}-01 & 6.5920 \mathrm{E}-01 & 7.4536 \mathrm{E}-01 \\ -38.45 & 8.2150 \mathrm{E}-01 & 8.0343 \mathrm{E}-01 & 8.3607 \mathrm{E}-01 \\ -33.32 & 9.2964 \mathrm{E}-01 & 9.2572 \mathrm{E}-01 & 9.3588 \mathrm{E}-01 \\ -28.20 & 1.0238 \mathrm{E}+00 & 1.0271 \mathrm{E}+00 & 1.0191 \mathrm{E}+00 \\ -23.07 & 1.1021 \mathrm{E}+00 & 1.1093 \mathrm{E}+00 & 1.0954 \mathrm{E}+00 \\ -17.94 & 1.1630 \mathrm{E}+00 & 1.1721 \mathrm{E}+00 & 1.1550 \mathrm{E}+00 \\ -12.82 & 1.2055 \mathrm{E}+00 & 1.2153 \mathrm{E}+00 & 1.1922 \mathrm{E}+00 \\ -7.69 & 1.2297 \mathrm{E}+00 & 1.2398 \mathrm{E}+00 & 1.2158 \mathrm{E}+00 \\ -2.56 & 1.2352 \mathrm{E}+00 & 1.2455 \mathrm{E}+00 & 1.2210 \mathrm{E}+00 \\ 2.56 & 1.2220 \mathrm{E}+00 & 1.2326 \mathrm{E}+00 & 1.2093 \mathrm{E}+00 \\ 7.69 & 1.1905 \mathrm{E}+00 & 1.2012 \mathrm{E}+00 & 1.1780 \mathrm{E}+00 \\ 12.82 & 1.1414 \mathrm{E}+00 & 1.1521 \mathrm{E}+00 & 1.1295 \mathrm{E}+00 \\ 17.94 & 1.0760 \mathrm{E}+00 & 1.0866 \mathrm{E}+00 & 1.0712 \mathrm{E}+00 \\ 23.07 & 9.9568 \mathrm{E}-01 & 1.0049 \mathrm{E}+00 & 9.9175 \mathrm{E}-01 \\ 28.20 & 9.0251 \mathrm{E}-01 & 9.0872 \mathrm{E}-01 & 9.0001 \mathrm{E}-01 \\ 33.32 & 7.9883 \mathrm{E}-01 & 7.9945 \mathrm{E}-01 & 8.0582 \mathrm{E}-01 \\ 38.45 & 6.8696 \mathrm{E}-01 & 6.7663 \mathrm{E}-01 & 6.9894 \mathrm{E}-01 \\ 43.58 & 5.7129 \mathrm{E}-01 & 5.4042 \mathrm{E}-01 & 5.9957 \mathrm{E}-01 \\ 47.17 & 4.9244 \mathrm{E}-01 & 4.3359 \mathrm{E}-01 & 5.1272 \mathrm{E}-02 \\ 50.62 & 4.1801 \mathrm{E}-01 & 3.3996 \mathrm{E}-01 & 3.6490 \mathrm{E}-02 \\ 55.49 & 3.2652 \mathrm{E}-01 & 2.4210 \mathrm{E}-01 & 2.4061 \mathrm{E}-02 \\ 60.35 & 2.4752 \mathrm{E}-01 & 1.6762 \mathrm{E}-01 & 1.6271 \mathrm{E}-02 \\ 64.98 & 1.9921 \mathrm{E}-01 & 1.2532 \mathrm{E}-01 & 0.0000 \mathrm{E}+00 \\ 69.39 & 1.7106 \mathrm{E}-01 & 1.0316 \mathrm{E}-01 & 0.0000 \mathrm{E}+00 \\ 76.46 & 1.3080 \mathrm{E}-01 & 7.3480 \mathrm{E}-02 & 0.0000 \mathrm{E}+00 \\ 86.80 & 8.5843 \mathrm{E}-02 & 4.4494 \mathrm{E}-02 & 0.0000 \mathrm{E}+00 \\ 98.14 & 4.9755 \mathrm{E}-02 & 2.4403 \mathrm{E}-02 & 0.0000 \mathrm{E}+00 \\ 110.00 & 2.1386 \mathrm{E}-02 & 1.0973 \mathrm{E}-02 & 0.0000 \mathrm{E}+00 \\ & & & \end{array}$


Table E10A-2.4. Axial Distribution of Total Flux, Fast Flux and Power in MFA-2 at EOC 10A-2

$\begin{array}{rccc}\text { z }(\mathrm{cm}) & \text { Total Flux } & \text { Flux }>0.1 \text { MeV } & \text { Power } \\ -97.78 & 2.5349 \mathrm{E}-02 & 1.2945 \mathrm{E}-02 & 0.0000 \mathrm{E}+00 \\ -92.78 & 5.4434 \mathrm{E}-02 & 2.4983 \mathrm{E}-02 & 0.0000 \mathrm{E}+00 \\ -87.78 & 8.7272 \mathrm{E}-02 & 3.9980 \mathrm{E}-02 & 0.0000 \mathrm{E}+00 \\ -82.78 & 1.2645 \mathrm{E}-01 & 5.9692 \mathrm{E}-02 & 0.0000 \mathrm{E}+00 \\ -77.78 & 1.7469 \mathrm{E}-01 & 8.6329 \mathrm{E}-02 & 0.0000 \mathrm{E}+00 \\ -72.15 & 2.2993 \mathrm{E}-01 & 1.2108 \mathrm{E}-01 & 0.0000 \mathrm{E}+00 \\ -65.90 & 2.8665 \mathrm{E}-01 & 1.6581 \mathrm{E}-01 & 0.0000 \mathrm{E}+00 \\ -60.35 & 3.4811 \mathrm{E}-01 & 2.2692 \mathrm{E}-01 & 2.5961 \mathrm{E}-02 \\ -55.49 & 4.2973 \mathrm{E}-01 & 3.0996 \mathrm{E}-01 & 3.4706 \mathrm{E}-02 \\ -50.62 & 5.2628 \mathrm{E}-01 & 4.1962 \mathrm{E}-01 & 4.8895 \mathrm{E}-02 \\ -47.17 & 6.0515 \mathrm{E}-01 & 5.2453 \mathrm{E}-01 & 6.5833 \mathrm{E}-02 \\ -43.58 & 6.8856 \mathrm{E}-01 & 6.4442 \mathrm{E}-01 & 7.2753 \mathrm{E}-01 \\ -38.45 & 8.0791 \mathrm{E}-01 & 7.8995 \mathrm{E}-01 & 8.2213 \mathrm{E}-01 \\ -33.32 & 9.1826 \mathrm{E}-01 & 9.1432 \mathrm{E}-01 & 9.2443 \mathrm{E}-01 \\ -28.20 & 1.0147 \mathrm{E}+00 & 1.0181 \mathrm{E}+00 & 1.0099 \mathrm{E}+00 \\ -23.07 & 1.0954 \mathrm{E}+00 & 1.1028 \mathrm{E}+00 & 1.0885 \mathrm{E}+00 \\ -17.94 & 1.1588 \mathrm{E}+00 & 1.1682 \mathrm{E}+00 & 1.1507 \mathrm{E}+00 \\ -12.82 & 1.2039 \mathrm{E}+00 & 1.2140 \mathrm{E}+00 & 1.1906 \mathrm{E}+00 \\ -7.69 & 1.2301 \mathrm{E}+00 & 1.2405 \mathrm{E}+00 & 1.2163 \mathrm{E}+00 \\ -2.56 & 1.2373 \mathrm{E}+00 & 1.2479 \mathrm{E}+00 & 1.2232 \mathrm{E}+00 \\ 2.56 & 1.2254 \mathrm{E}+00 & 1.2363 \mathrm{E}+00 & 1.2127 \mathrm{E}+00 \\ 7.69 & 1.1952 \mathrm{E}+00 & 1.2062 \mathrm{E}+00 & 1.1828 \mathrm{E}+00 \\ 12.82 & 1.1475 \mathrm{E}+00 & 1.1584 \mathrm{E}+00 & 1.1358 \mathrm{E}+00 \\ 17.94 & 1.0836 \mathrm{E}+00 & 1.0942 \mathrm{E}+00 & 1.0790 \mathrm{E}+00 \\ 23.07 & 1.0044 \mathrm{E}+00 & 1.0133 \mathrm{E}+00 & 1.0007 \mathrm{E}+00 \\ 28.20 & 9.1153 \mathrm{E}-01 & 9.1726 \mathrm{E}-01 & 9.0936 \mathrm{E}-01 \\ 33.32 & 8.0730 \mathrm{E}-01 & 8.0718 \mathrm{E}-01 & 8.1465 \mathrm{E}-01 \\ 38.45 & 6.9394 \mathrm{E}-01 & 6.8271 \mathrm{E}-01 & 7.0641 \mathrm{E}-01 \\ 43.58 & 5.7617 \mathrm{E}-01 & 5.4429 \mathrm{E}-01 & 6.0530 \mathrm{E}-01 \\ 47.17 & 4.9576 \mathrm{E}-01 & 4.3590 \mathrm{E}-01 & 5.2414 \mathrm{E}-02 \\ 50.62 & 4.2009 \mathrm{E}-01 & 3.4124 \mathrm{E}-01 & 3.7362 \mathrm{E}-02 \\ 55.49 & 3.2781 \mathrm{E}-01 & 2.4279 \mathrm{E}-01 & 2.4686 \mathrm{E}-02 \\ 60.35 & 2.4873 \mathrm{E}-01 & 1.6818 \mathrm{E}-01 & 1.6733 \mathrm{E}-02 \\ 64.98 & 2.0033 \mathrm{E}-01 & 1.2556 \mathrm{E}-01 & 0.0000 \mathrm{E}+00 \\ 69.39 & 1.7167 \mathrm{E}-01 & 1.0295 \mathrm{E}-01 & 0.0000 \mathrm{E}+00 \\ 76.46 & 1.3050 \mathrm{E}-01 & 7.2717 \mathrm{E}-02 & 0.0000 \mathrm{E}+00 \\ 86.80 & 8.5047 \mathrm{E}-02 & 4.3565 \mathrm{E}-02 & 0.0000 \mathrm{E}+00 \\ 98.14 & 4.89315 \mathrm{E}-02 & 2.3625 \mathrm{E}-02 & 0.0000 \mathrm{E}+00 \\ 110.00 & 2.0735 \mathrm{E}-02 & 1.0447 \mathrm{E}-02 & 0.0000 \mathrm{E}+00\end{array}$


Tab1e E10A-2.5. Fission Power Distribution by Pin in MFA-1 at EOC 10A-2

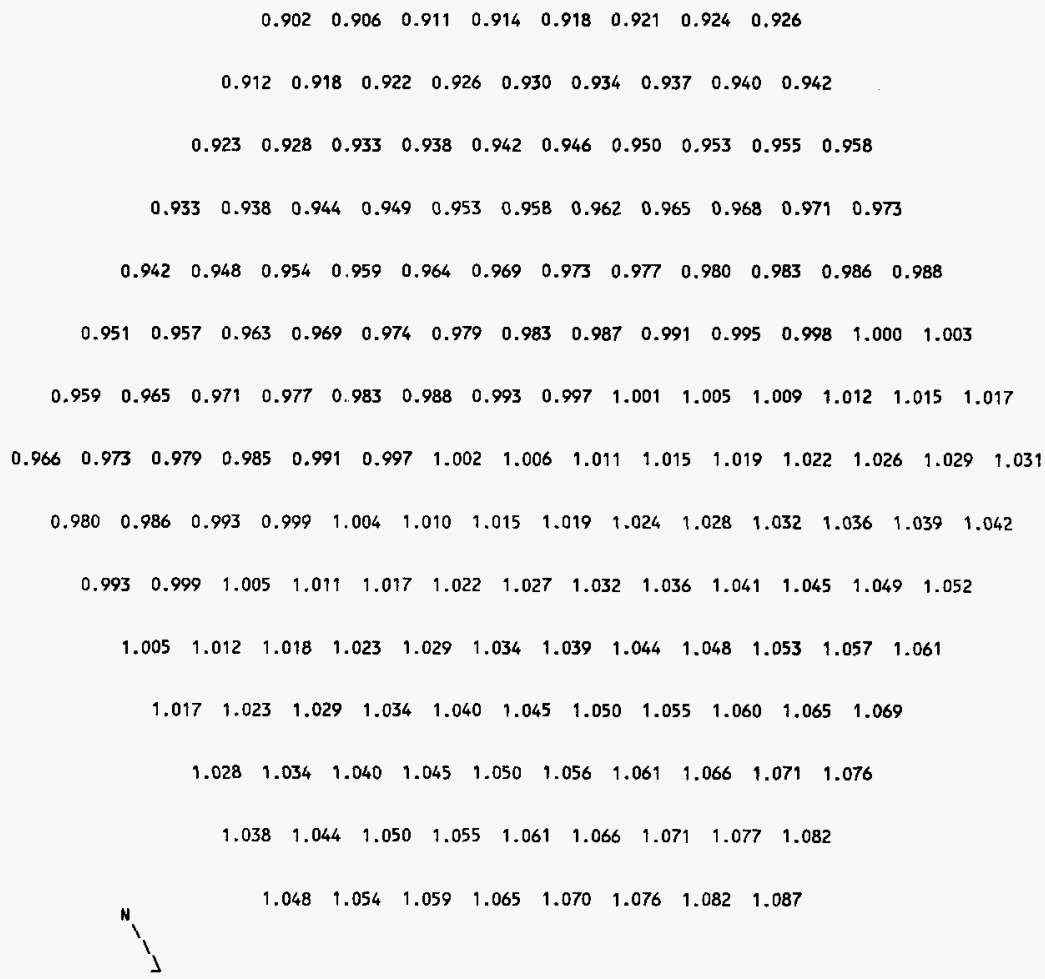


Table E1OA-2.6. Fission Power Distribution by Pin in MFA-2 at EOC 10A-2

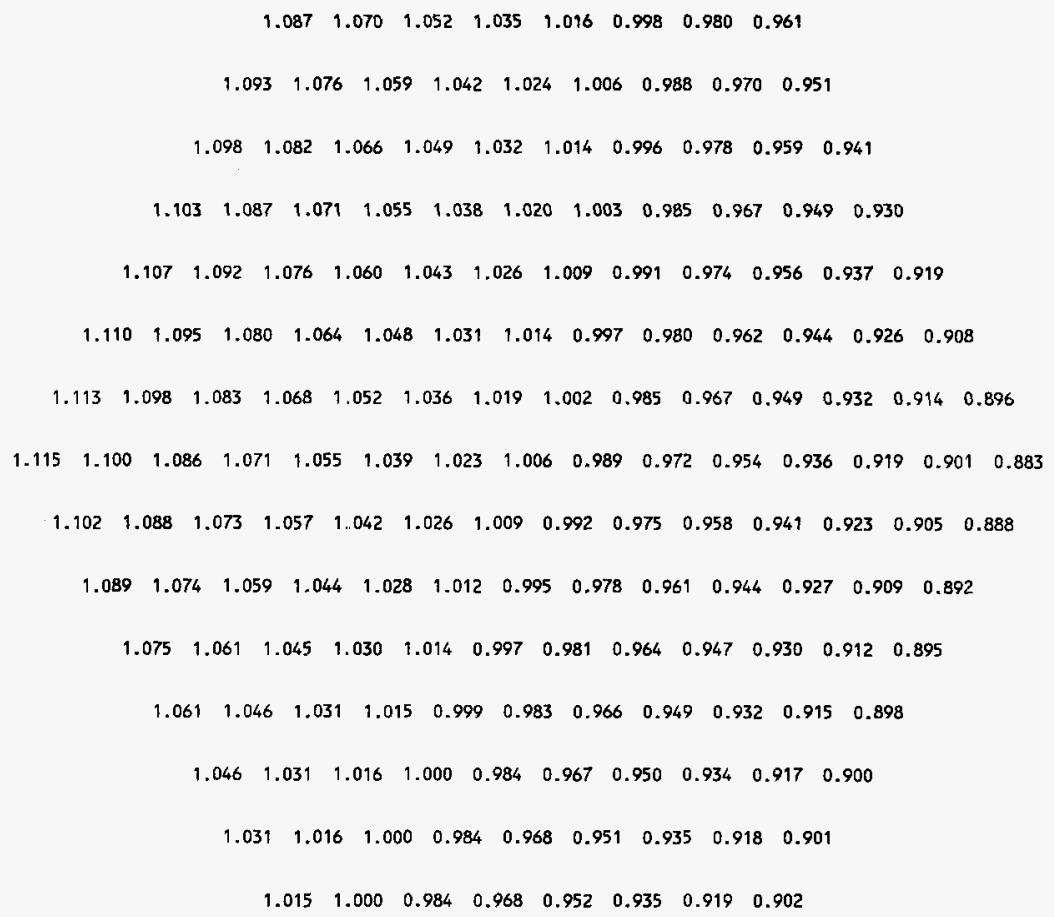


Table E1OA-2.7. Total Flux Distribution by Pin in MFA-1 at EOC 10A-2

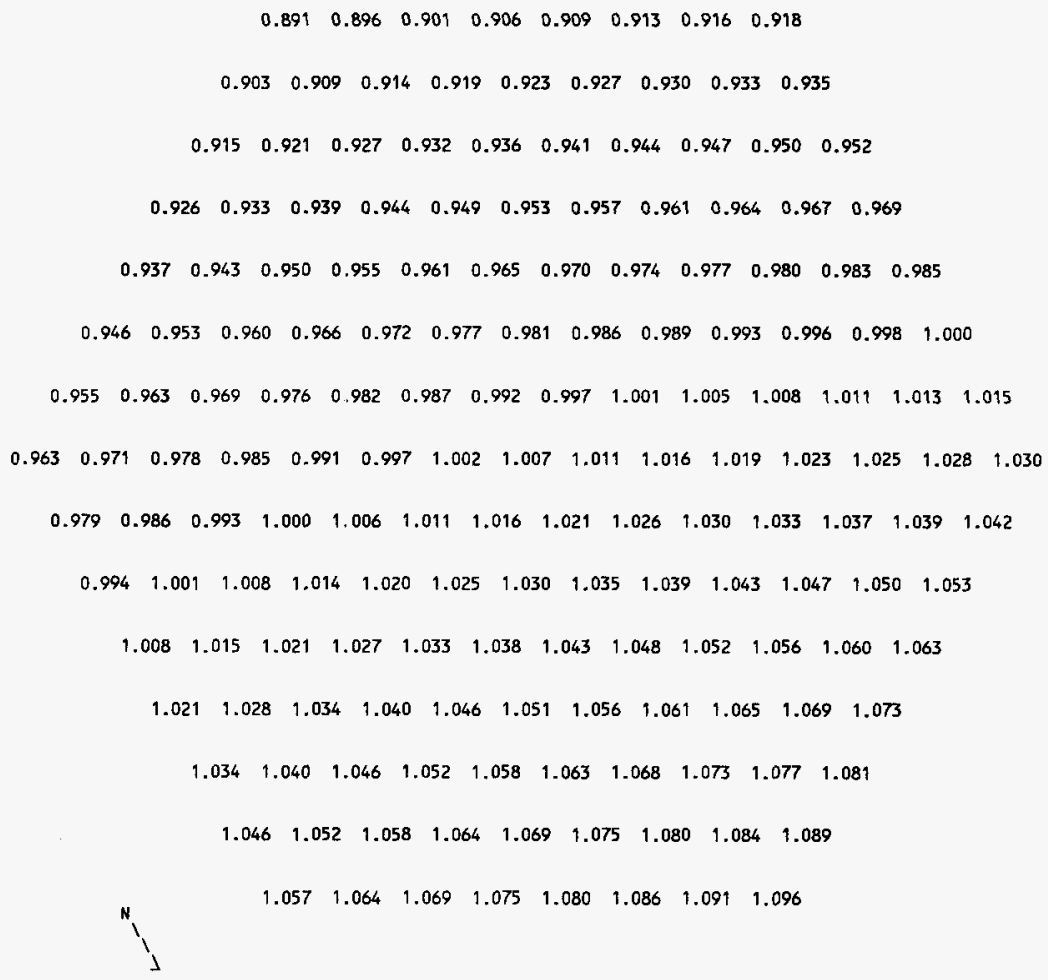


Table E10A-2.8. Total Flux Distribution by Pin in MFA-2 at EOC 10A-2

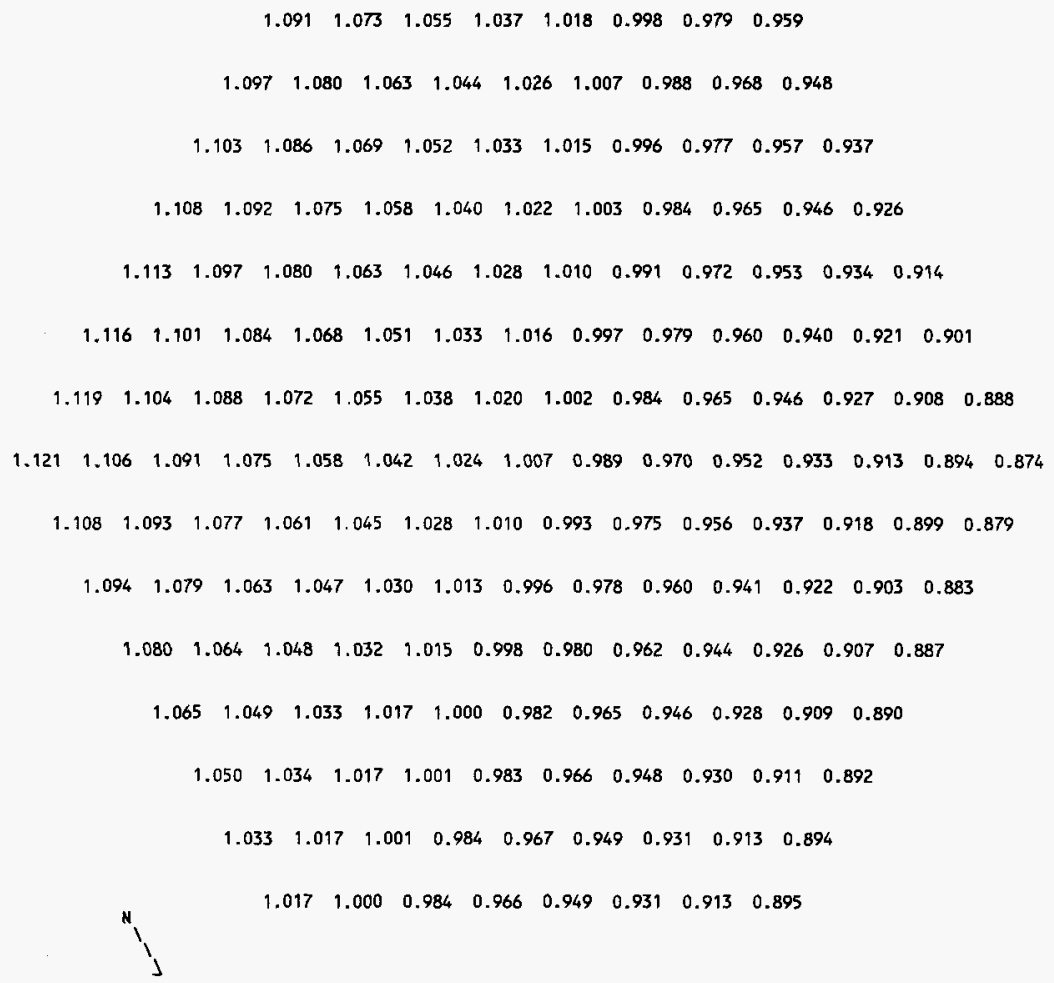


Table E10A-2.9. MFA-1 and MFA-2 Duct WalT Flux Greater Than $0.1 \mathrm{MeV}$ at EOC 10A-2

$\begin{array}{ccc}\text { SIDE } & \begin{array}{c}\text { FLUX > 0.1 } \\ \text { MFA-1 }\end{array} & \begin{array}{c}\text { MEV } \\ \left(\mathrm{r} / / \mathrm{cm}^{2}-\mathrm{sec}\right) \\ \text { NFA-2 }\end{array} \\ \text { E } & 1.8766 \mathrm{E}+15 & 2.14 .39 \mathrm{E}+15 \\ \text { SE } & 1.6775 \mathrm{E}+15 & 2.2302 \mathrm{E}+15 \\ \text { SW } & 1.6097 \mathrm{E}+15 & 2.0223 \mathrm{E}+15 \\ \text { W } & 1.7710 \mathrm{E}+15 & 1.7649 \mathrm{E}+15 \\ \text { NW } & 1.9890 \mathrm{E}+15 & 1.6878 \mathrm{E}+15 \\ \text { NE } & 2.0436 \mathrm{E}+15 & 1.8463 \mathrm{E}+15\end{array}$

Table E1OA-2.10. Assembly Outlet Temperatures and Flow Rates at EOC 10A-2

$\begin{array}{lcl} & \text { CALCULATED } \\ \text { CORE } & \text { OUTLET TEMP. } & \text { FLOW RATE } \\ \text { POS. } & \text { (DEG F) } & \text { (LB/H) } \\ 1506 & 1032 & 116330 \\ 1404 & 907 & 228160 \\ 1405 & 881 & 210640 \\ 1505 & 869 & 210640 \\ 1507 & 851 & 210640 \\ 1606 & 898 & 172740 \\ 1607 & 860 & 172740 \\ 2507 & 1052 & 172740 \\ 2405 & 929 & 194730 \\ 2506 & 888 & 200300 \\ 2508 & 914 & 210540 \\ 2607 & 904 & 165560 \\ 2608 & 893 & 89760 \\ 2609 & 890 & 175430\end{array}$




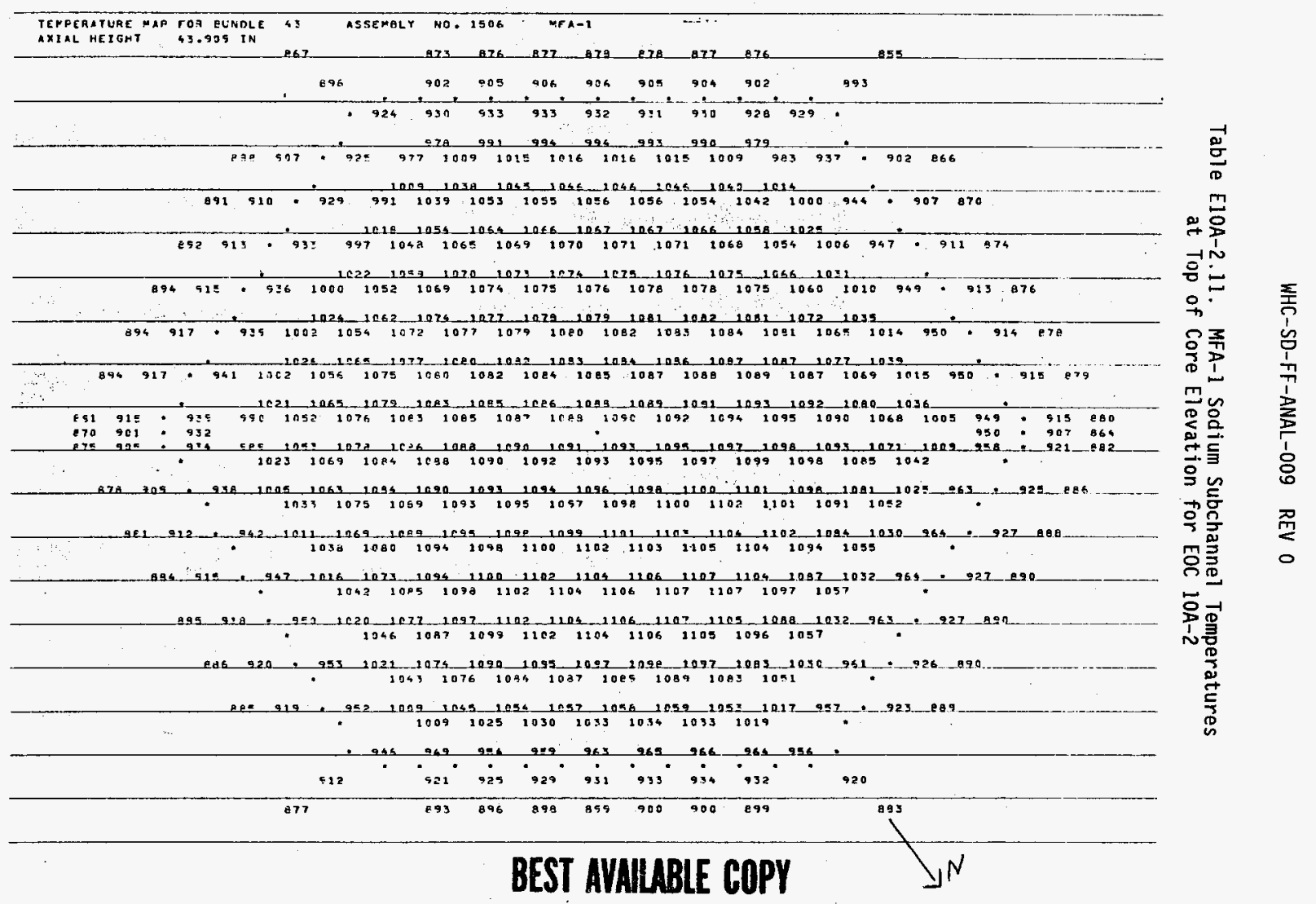


Table E10A-2.12. MFA-2 Sodium Subchannel Temperatures at Top of Core Elevation for EOC 1OA-2

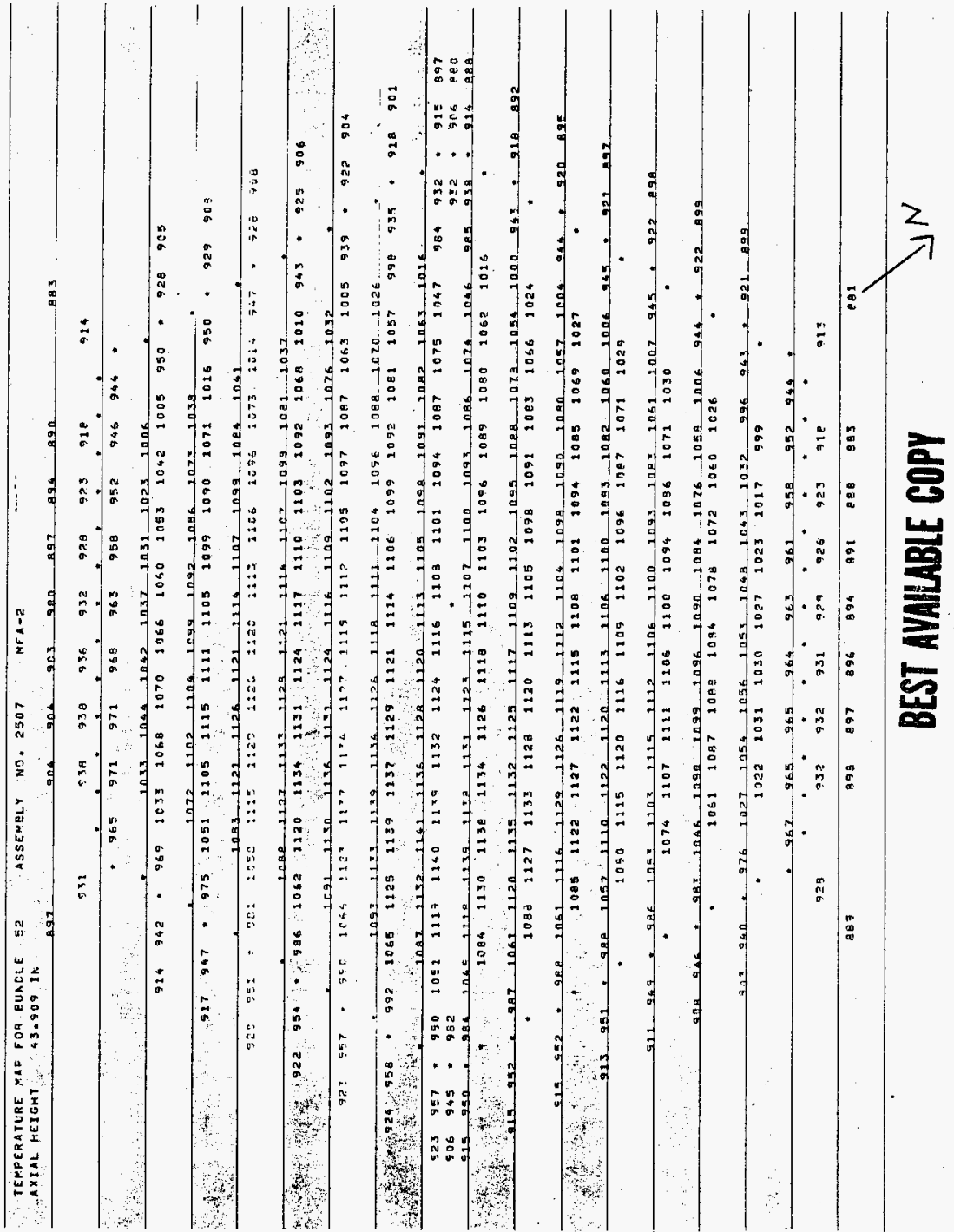


Table E10A-2.13. MFA-1 Sodium Subchannel Temperatures at Elevation of Upper Axial B 1 anket for EOC $10 \mathrm{~A}-2$

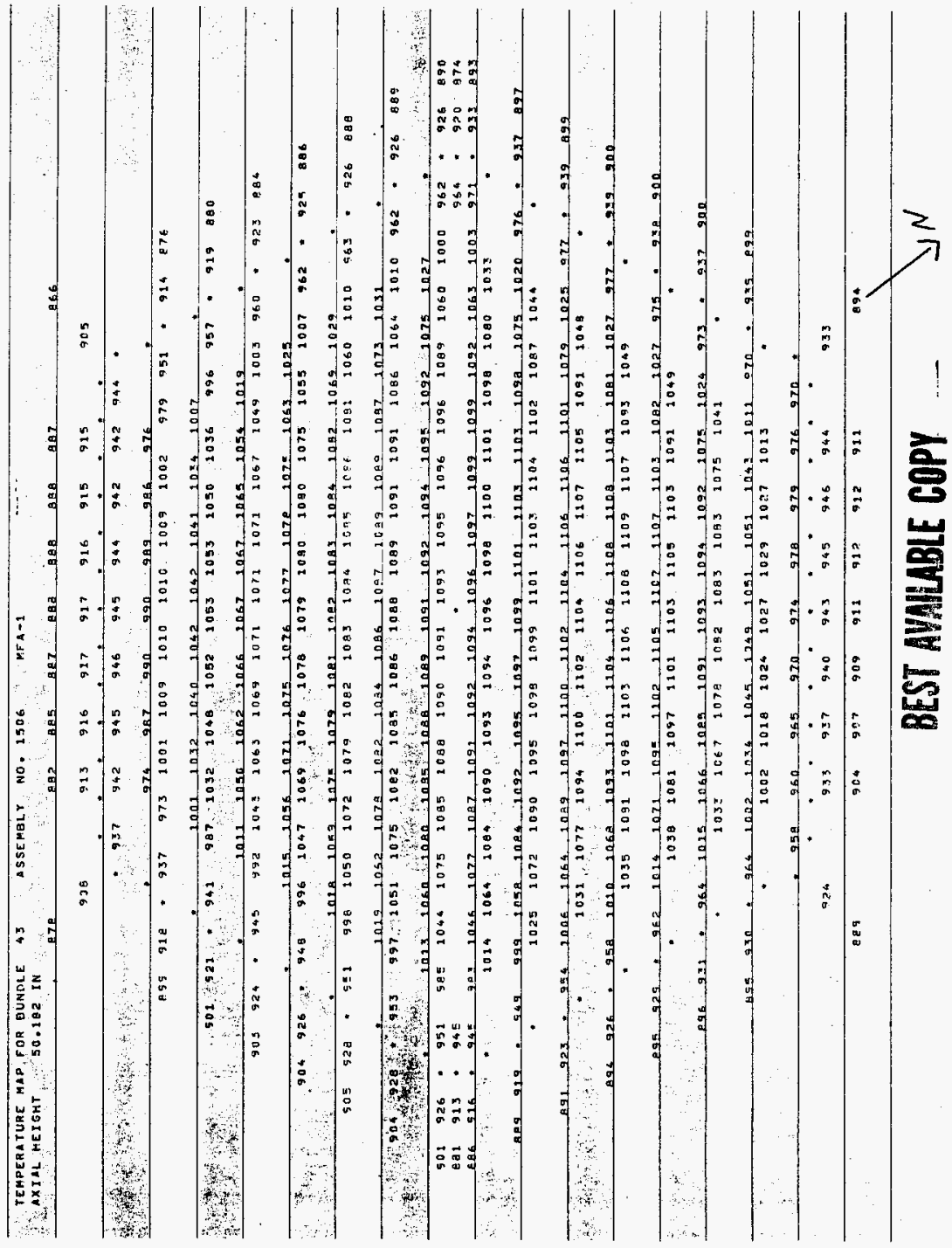




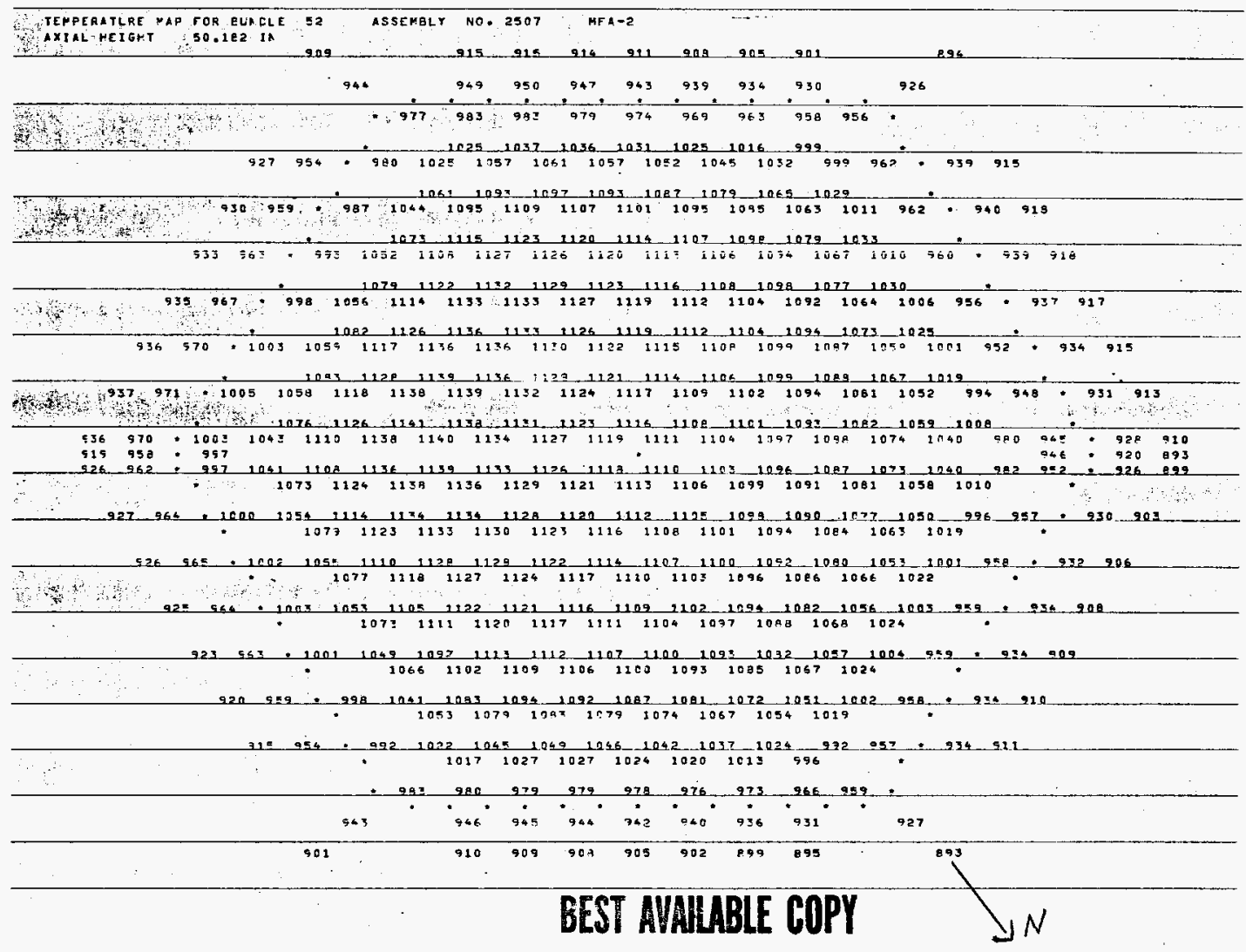




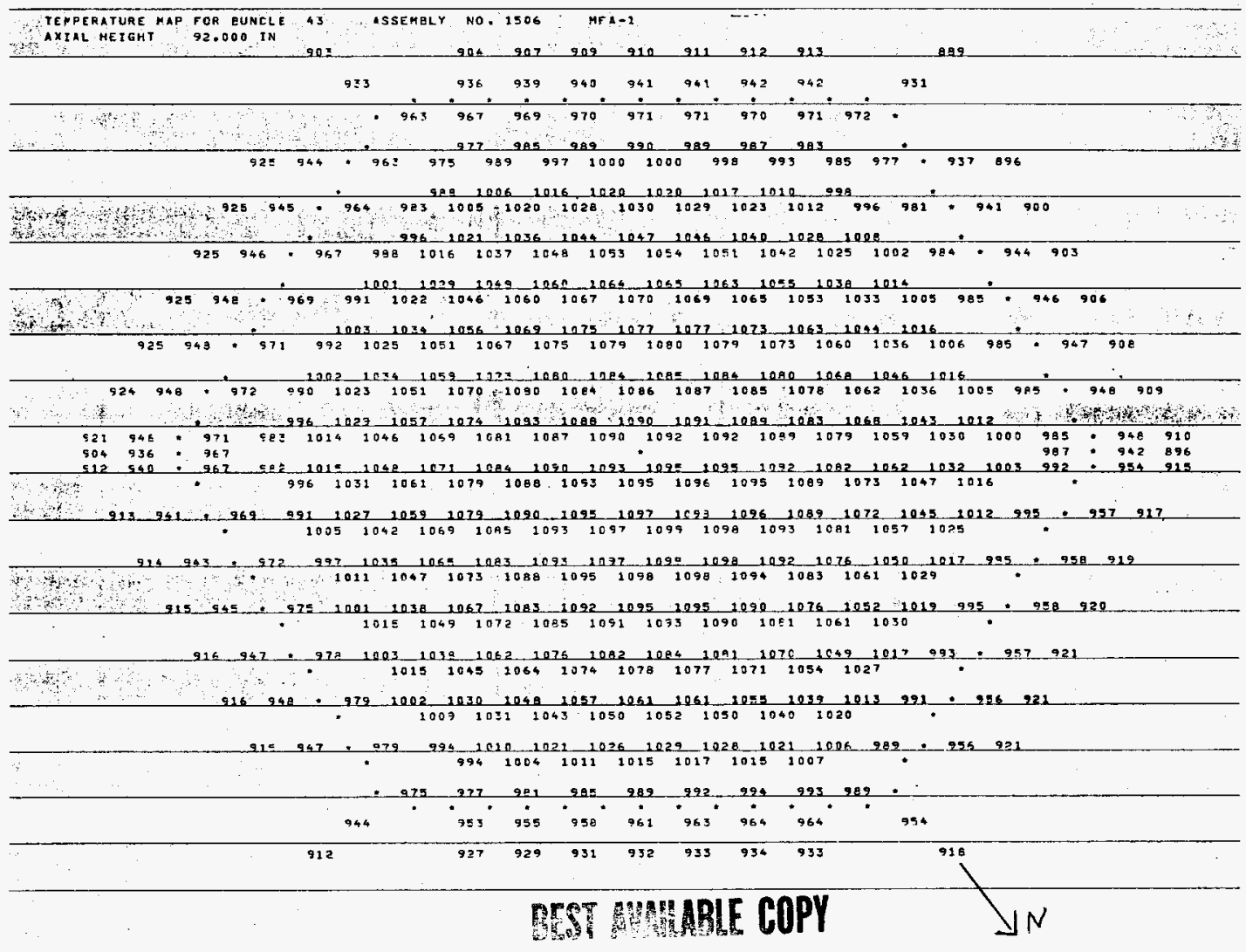


Table E10A-2.16. MFA-2 Sodium Subchannel Temperatures at Top of Fuel Pin Bundle for EOC 10A-2

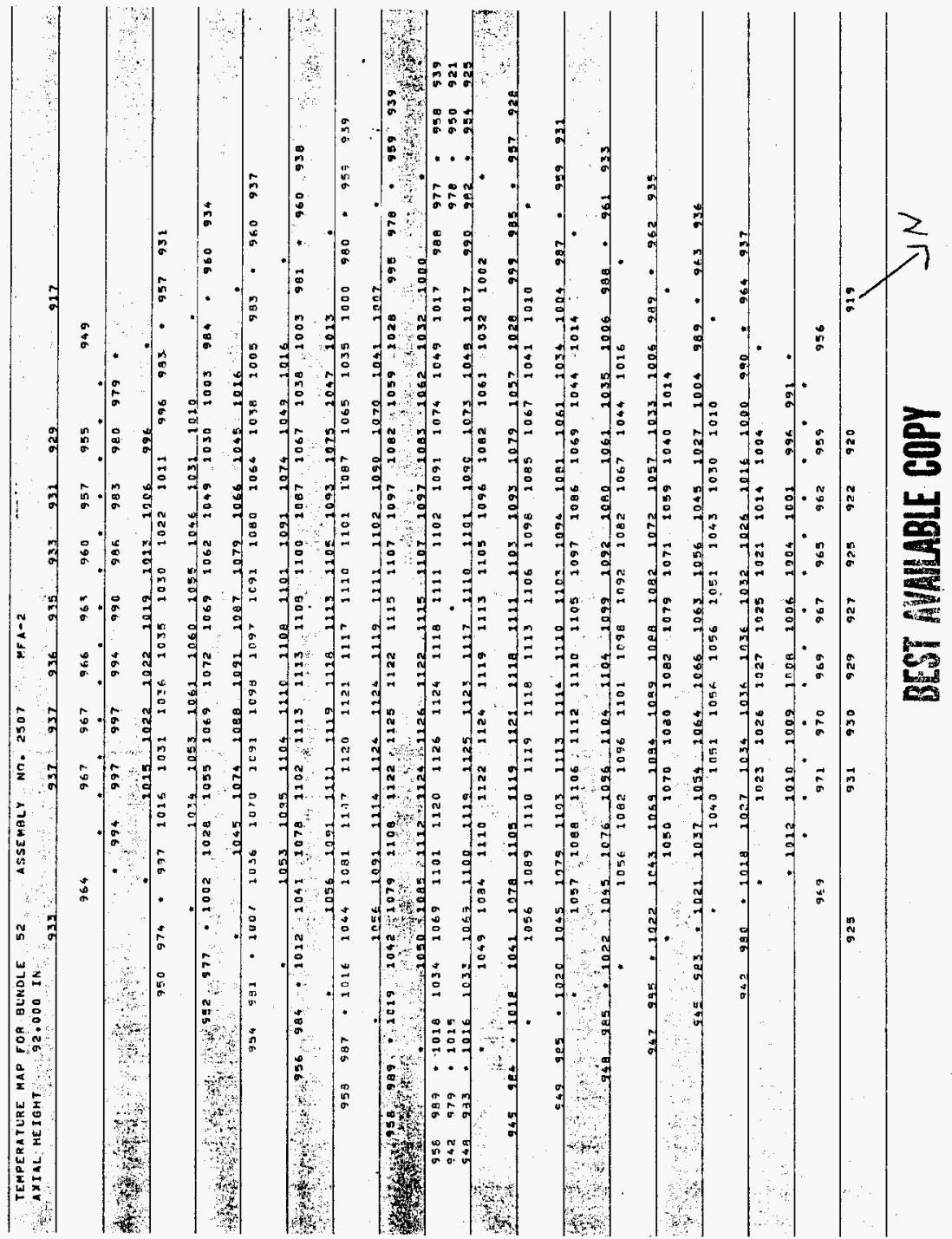


Table ElOA-2.17. Composition of MFA-1 at EOC 10A-2

\begin{tabular}{|c|c|c|c|c|c|}
\hline Axial & Axial & $(\mathrm{cm})$ & & Atom Den & $y(a / b-c m)$ \\
\hline $\begin{array}{c}\text { Leve } 1 \\
1\end{array}$ & $\begin{array}{r}\text { Lower } \\
-100.28\end{array}$ & $\begin{array}{r}\text { Upper } \\
-75.28\end{array}$ & Constituent & Fresh & End of Cycle \\
\hline & & & $\begin{array}{l}\mathrm{Na}-23 \\
\mathrm{SS}-316\end{array}$ & $\begin{array}{l}5.3150 E-03 \\
6.4090 E-02\end{array}$ & $\begin{array}{l}5.3150 \mathrm{E}-03 \\
6.4090 \mathrm{E}-02\end{array}$ \\
\hline 2 & -75.28 & -62.78 & & & \\
\hline & & & $\begin{array}{l}\mathrm{Na}-23 \\
\mathrm{SS}-316\end{array}$ & $\begin{array}{l}1.3177 \mathrm{E}-02 \\
3.4175 \mathrm{E}-02\end{array}$ & $\begin{array}{l}1.3177 \mathrm{E}-02 \\
3.4175 \mathrm{E}-02\end{array}$ \\
\hline 3 & -62.78 & -46.14 & & & \\
\hline & & & $\begin{array}{l}\mathrm{U}-235 \\
\mathrm{U}-238 \\
\mathrm{Pu}-239 \\
\mathrm{Pu}-240 \\
\mathrm{FP} \\
0-16 \\
\mathrm{Na}-23 \\
\mathrm{SS}-316\end{array}$ & $\begin{array}{l}1.4143 \mathrm{E}-05 \\
7.0573 \mathrm{E}-03 \\
0.0000 \mathrm{E}+00 \\
0.0000 \mathrm{E}+00 \\
0.0000 \mathrm{E}+00 \\
1.4143 \mathrm{E}-02 \\
9.7416 \mathrm{E}-03 \\
1.9073 \mathrm{E}-02\end{array}$ & $\begin{array}{l}1.3496 \mathrm{E}-05 \\
7.0246 \mathrm{E}-03 \\
3.0158 \mathrm{E}-05 \\
1.9609 \mathrm{E}-07 \\
2.7995 \mathrm{E}-06 \\
1.4143 \mathrm{E}-02 \\
9.7416 \mathrm{E}-03 \\
1.9073 \mathrm{E}-02\end{array}$ \\
\hline 4 & -46.14 & -30.76 & & & \\
\hline & & & $\begin{array}{l}\mathrm{U}-235 \\
\mathrm{U}-238 \\
\mathrm{Pu}-239 \\
\mathrm{Pu}-240 \\
\mathrm{Pu}-241 \\
\mathrm{Pu}-242 \\
\mathrm{Am}-241 \\
\mathrm{FP} \\
\mathrm{O}-16 \\
\mathrm{Na}-23 \\
\mathrm{SS}-316\end{array}$ & $\begin{array}{l}1.0840 \mathrm{E}-05 \\
4.4499 \mathrm{E}-03 \\
1.7520 \mathrm{E}-03 \\
2.2340 \mathrm{E}-04 \\
1.9280 \mathrm{E}-05 \\
4.7430 \mathrm{E}-06 \\
7.6680 \mathrm{E}-07 \\
0.0000 \mathrm{E}+00 \\
1.2670 \mathrm{E}-02 \\
9.8100 \mathrm{E}-03 \\
1.8990 \mathrm{E}-02\end{array}$ & $\begin{array}{l}1.0386 \mathrm{E}-05 \\
4.4246 \mathrm{E}-03 \\
1.7006 \mathrm{E}-03 \\
2.3350 \mathrm{E}-04 \\
1.9937 \mathrm{E}-05 \\
4.7949 \mathrm{E}-06 \\
9.7032 \mathrm{E}-07 \\
6.5884 \mathrm{E}-05 \\
1.2670 \mathrm{E}-02 \\
9.8100 \mathrm{E}-03 \\
1.8990 \mathrm{E}-02\end{array}$ \\
\hline 5 & -30.76 & 15.38 & & & \\
\hline & & & $\begin{array}{l}\mathrm{U}-235 \\
\mathrm{U}-238 \\
\mathrm{Pu}-239 \\
\mathrm{Pu}-240 \\
\mathrm{Pu}-241 \\
\mathrm{Pu}-242 \\
\mathrm{Am}-241 \\
\mathrm{FP} \\
\mathrm{O}-16 \\
\mathrm{Na}-23 \\
\mathrm{SS}-316\end{array}$ & $\begin{array}{l}1.0840 \mathrm{E}-05 \\
4.4499 \mathrm{E}-03 \\
1.7520 \mathrm{E}-03 \\
2.2340 \mathrm{E}-04 \\
1.9280 \mathrm{E}-05 \\
4.7430 \mathrm{E}-06 \\
7.6680 \mathrm{E}-07 \\
0.0000 \mathrm{E}+00 \\
1.2670 \mathrm{E}-02 \\
9.8100 \mathrm{E}-03 \\
1.8990 \mathrm{E}-02\end{array}$ & $\begin{array}{l}1.0273 \mathrm{E}-05 \\
4.4177 \mathrm{E}-03 \\
1.6860 \mathrm{E}-03 \\
2.3458 \mathrm{E}-04 \\
1.9985 \mathrm{E}-05 \\
4.7950 \mathrm{E}-06 \\
9.6380 \mathrm{E}-07 \\
8.6461 \mathrm{E}-05 \\
1.2670 \mathrm{E}-02 \\
9.8100 \mathrm{E}-03 \\
1.8990 \mathrm{E}-02\end{array}$ \\
\hline 6 & -15.38 & 0.00 & & & \\
\hline & & & $\begin{array}{l}\mathrm{U}-235 \\
\mathrm{U}-238 \\
\mathrm{Pu}-239 \\
\mathrm{Pu}-240 \\
\mathrm{Pu}-241 \\
\mathrm{Pu}-242 \\
\mathrm{Am}-241 \\
\mathrm{FP} \\
\mathrm{O}-16 \\
\mathrm{Na}-23 \\
\mathrm{SS}-316\end{array}$ & $\begin{array}{l}1.0840 \mathrm{E}-05 \\
4.4499 \mathrm{E}-03 \\
1.7520 \mathrm{E}-03 \\
2.2340 \mathrm{E}-04 \\
1.9280 \mathrm{E}-05 \\
4.7430 \mathrm{E}-06 \\
7.6680 \mathrm{E}-07 \\
0.0000 \mathrm{E}+00 \\
1.2670 \mathrm{E}-02 \\
9.8100 \mathrm{E}-03 \\
1.8990 \mathrm{E}-02\end{array}$ & $\begin{array}{l}1.0210 \mathrm{E}-05 \\
4.4140 \mathrm{E}-03 \\
1.6785 \mathrm{E}-03 \\
2.3571 \mathrm{E}-04 \\
2.0084 \mathrm{E}-05 \\
4.8005 \mathrm{E}-06 \\
9.6042 \mathrm{E}-07 \\
9.6417 \mathrm{E}-05 \\
1.2670 \mathrm{E}-02 \\
9.8100 \mathrm{E}-03 \\
1.8990 \mathrm{E}-02\end{array}$ \\
\hline
\end{tabular}


$0.00 \quad 15.38$

$\begin{array}{lll}8 & 15.38 & 30.76\end{array}$

$\begin{array}{lll}\text { U-235 } & 1.0840 \mathrm{E}-05 & 1.0230 \mathrm{E}-05 \\ \mathrm{U}-238 & 4.4499 \mathrm{E}-03 & 4.4152 \mathrm{E}-03 \\ \text { PU-239 } & 1.7520 \mathrm{E}-03 & 1.6808 \mathrm{E}-03 \\ \text { Pu-240 } & 2.2340 \mathrm{E}-04 & 2.3529 \mathrm{E}-04 \\ \text { Pu-241 } & 1.9280 \mathrm{E}-05 & 2.0046 \mathrm{E}-05 \\ \text { PU-242 } & 4.7430 \mathrm{E}-06 & 4.7983 \mathrm{E}-06 \\ \mathrm{Am}-241 & 7.6680 \mathrm{E}-07 & 9.6151 \mathrm{E}-07 \\ \mathrm{FP} & 0.0000 \mathrm{E}+00 & 9.3286 \mathrm{E}-05 \\ 0-16 & 1.2670 \mathrm{E}-02 & 1.2670 \mathrm{E}-02 \\ \mathrm{Na}-23 & 9.8100 \mathrm{E}-03 & 9.8100 \mathrm{E}-03 \\ \mathrm{SS}-316 & 1.8990 \mathrm{E}-02 & 1.8990 \mathrm{E}-02\end{array}$

$\begin{array}{lll}\mathrm{U}-235 & 1.0840 \mathrm{E}-05 & 1.0329 \mathrm{E}-05 \\ \mathrm{U}-238 & 4.4499 \mathrm{E}-03 & 4.4209 \mathrm{E}-03 \\ \mathrm{Pu}-239 & 1.7520 \mathrm{E}-03 & 1.6925 \mathrm{E}-03 \\ \mathrm{Pu}-240 & 2.2340 \mathrm{E}-04 & 2.3341 \mathrm{E}-04 \\ \mathrm{PU}-241 & 1.9280 \mathrm{E}-05 & 1.9881 \mathrm{E}-05 \\ \mathrm{PU}-242 & 4.7430 \mathrm{E}-06 & 4.7888 \mathrm{E}-06 \\ \mathrm{Am}-241 & 7.6680 \mathrm{E}-07 & 9.6677 \mathrm{E}-07 \\ \mathrm{FP} & 0.0000 \mathrm{E}+00 & 7.8016 \mathrm{E}-05 \\ \mathrm{O}-16 & 1.2670 \mathrm{E}-02 & 1.2670 \mathrm{E}-02 \\ \mathrm{Na}-23 & 9.8100 \mathrm{E}-03 & 9.8100 \mathrm{E}-03 \\ \mathrm{SS}-316 & 1.8990 \mathrm{E}-02 & 1.8990 \mathrm{E}-02\end{array}$

$\begin{array}{lll}9 & 30.76 & 46.14\end{array}$

$\begin{array}{lll}\text { U-235 } & 1.0840 \mathrm{E}-05 & 1.0465 \mathrm{E}-05 \\ \mathrm{U}-238 & 4.4499 \mathrm{E}-03 & 4.4291 \mathrm{E}-03 \\ \mathrm{Pu}-239 & 1.7520 \mathrm{E}-03 & 1.7094 \mathrm{E}-03 \\ \mathrm{PU}-240 & 2.2340 \mathrm{E}-04 & 2.3152 \mathrm{E}-04 \\ \mathrm{Pu}-241 & 1.9280 \mathrm{E}-05 & 1.9747 \mathrm{E}-05 \\ \mathrm{Pu}-242 & 4.7430 \mathrm{E}-06 & 4.7832 \mathrm{E}-06 \\ \mathrm{Am}-241 & 7.6680 \mathrm{E}-07 & 9.7442 \mathrm{E}-07 \\ \mathrm{FP} & 0.0000 \mathrm{E}+00 & 5.4760 \mathrm{E}-05 \\ \mathrm{O}-16 & 1.2670 \mathrm{E}-02 & 1.2670 \mathrm{E}-02 \\ \mathrm{Na}-23 & 9.8100 \mathrm{E}-03 & 9.8100 \mathrm{E}-03 \\ \text { SS-316 } & 1.8990 \mathrm{E}-02 & 1.8990 \mathrm{E}-02\end{array}$

$\begin{array}{lll}10 & 46.14 & 62.78\end{array}$

$\begin{array}{lll}\text { U-235 } & 1.4143 \mathrm{E}-05 & 1.3709 \mathrm{E}-05 \\ \mathrm{U}-238 & 7.0573 \mathrm{E}-03 & 7.0347 \mathrm{E}-03 \\ \text { PU-239 } & 0.0000 \mathrm{E}+00 & 2.0819 \mathrm{E}-05 \\ \text { PU-240 } & 0.0000 \mathrm{E}+00 & 8.6890 \mathrm{E}-08 \\ \text { FP } & 0.0000 \mathrm{E}+00 & 2.0138 \mathrm{E}-06 \\ 0-16 & 1.4143 \mathrm{E}-02 & 1.4143 \mathrm{E}-02 \\ \mathrm{Na}-23 & 9.7416 \mathrm{E}-03 & 9.7416 \mathrm{E}-03 \\ \text { SS-316 } & 1.9073 \mathrm{E}-02 & 1.9073 \mathrm{E}-02\end{array}$

$11 \quad 62.78 \quad 116.00$

$\begin{array}{lll}\mathrm{Na}-23 & 8.2400 \mathrm{E}-03 & 8.2400 \mathrm{E}-03 \\ \mathrm{SS}-316 & 2.5562 \mathrm{E}-02 & 2.5562 \mathrm{E}-02\end{array}$


Table E1OA-2.18. Composition of MFA-2 at EOC 1OA-2

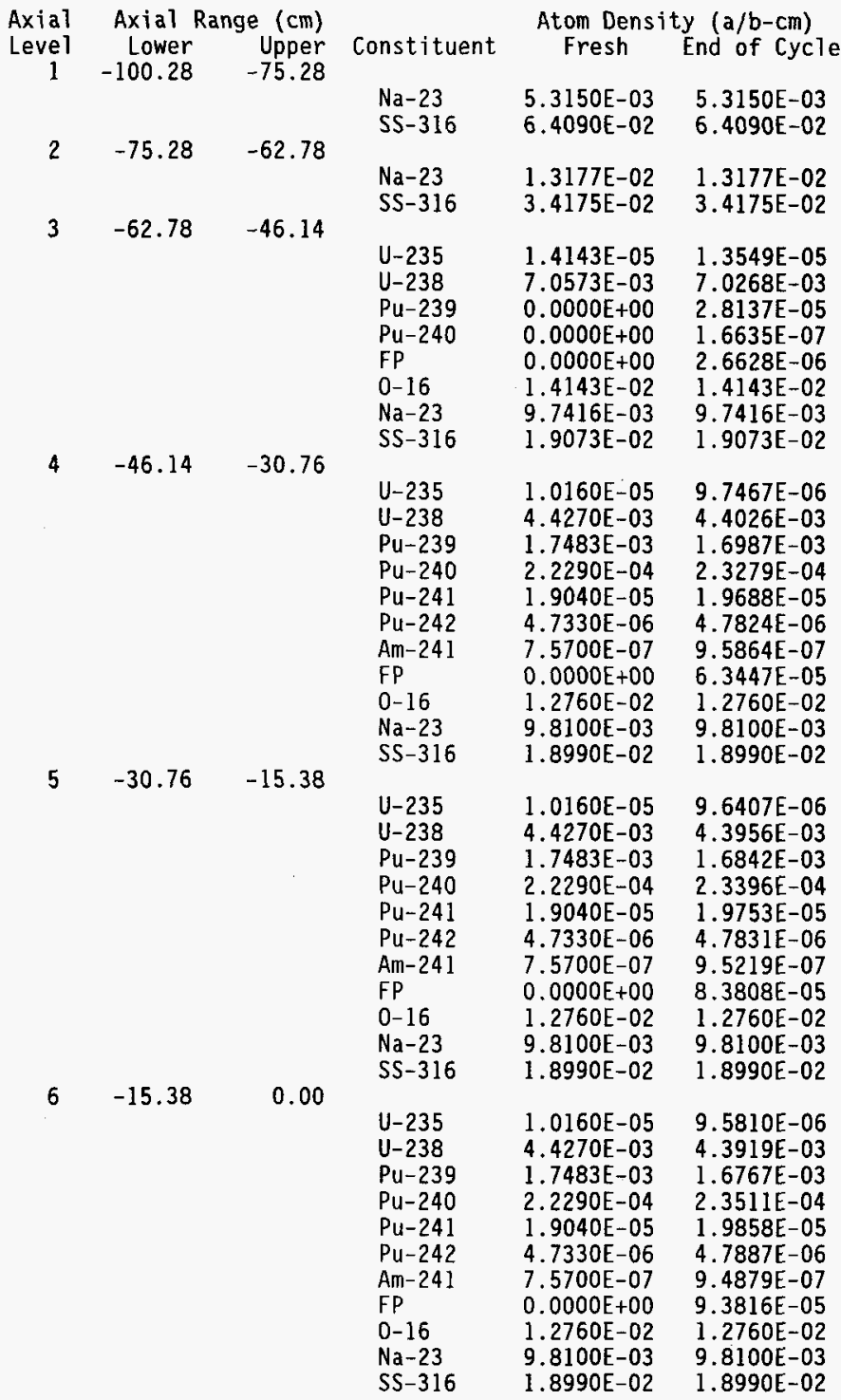


$\begin{array}{lll}7 & 0.00 & 15.38\end{array}$

$\begin{array}{lll}8 & 15.38 & 30.76\end{array}$

$\begin{array}{ll}\text { U-235 } & 1.0160 E-05 \\ \text { U-238 } & 4.4270 E-03 \\ \text { Pu-239 } & 1.7483 E-03 \\ \text { PU-240 } & 2.2290 E-04 \\ \text { Pu-241 } & 1.9040 E-05 \\ \text { Pu-242 } & 4.7330 E-06 \\ \text { Am-241 } & 7.5700 E-07 \\ \text { FP } & 0.0000 E+00 \\ 0-16 & 1.2760 E-02 \\ \text { Na-23 } & 9.8100 E-03 \\ \text { SS-316 } & 1.8990 E-02\end{array}$

9. 5986E-06

4.3930E-03

$1.6788 \mathrm{E}-03$

2. $3473 \mathrm{E}-04$

1. $9822 \mathrm{E}-05$

4.7867E-06

$9.4976 \mathrm{E}-07$

$9.0994 \mathrm{E}-05$

1.2760E-02

$9.8100 \mathrm{E}-03$

1.8990E-02

$\begin{array}{lll}\mathrm{U}-235 & 1.0160 \mathrm{E}-05 & 9.6866 \mathrm{E}-06 \\ \mathrm{U}-238 & 4.4270 \mathrm{E}-03 & 4.3984 \mathrm{E}-03 \\ \mathrm{Pu}-239 & 1.7483 \mathrm{E}-03 & 1.6898 \mathrm{E}-03 \\ \mathrm{PU}-240 & 2.2290 \mathrm{E}-04 & 2.3295 \mathrm{E}-04 \\ \mathrm{Pu}-241 & 1.9040 \mathrm{E}-05 & 1.9663 \mathrm{E}-05 \\ \mathrm{Pu}-242 & 4.7330 \mathrm{E}-06 & 4.7780 \mathrm{E}-06 \\ \mathrm{Am}-241 & 7.5700 \mathrm{E}-07 & 9.5474 \mathrm{E}-07 \\ \mathrm{FP} & 0.0000 \mathrm{E}+00 & 7.6462 \mathrm{E}-05 \\ 0-16 & 1.2760 \mathrm{E}-02 & 1.2760 \mathrm{E}-02 \\ \mathrm{Na}-23 & 9.8100 \mathrm{E}-03 & 9.8100 \mathrm{E}-03 \\ \mathrm{SS}-316 & 1.8990 \mathrm{E}-02 & 1.8990 \mathrm{E}-02\end{array}$

$9 \quad 30.76 \quad 46.14$

$\begin{array}{lll}\text { U-235 } & 1.0160 E-05 & 9.8117 \mathrm{E}-06 \\ \mathrm{U}-238 & 4.4270 \mathrm{E}-03 & 4.4064 \mathrm{E}-03 \\ \mathrm{Pu}-239 & 1.7483 \mathrm{E}-03 & 1.7064 \mathrm{E}-03 \\ \mathrm{Pu}-240 & 2.2290 \mathrm{E}-04 & 2.3109 \mathrm{E}-04 \\ \mathrm{Pu}-241 & 1.9040 \mathrm{E}-05 & 1.9525 \mathrm{E}-05 \\ \mathrm{Pu}-242 & 4.7330 \mathrm{E}-06 & 4.7726 \mathrm{E}-06 \\ \mathrm{Am}-241 & 7.5700 \mathrm{E}-07 & 9.6214 \mathrm{E}-07 \\ \mathrm{FP} & 0.0000 \mathrm{E}+00 & 5.3857 \mathrm{E}-05 \\ 0-16 & 1.2760 \mathrm{E}-02 & 1.2760 \mathrm{E}-02 \\ \mathrm{Na}-23 & 9.8100 \mathrm{E}-03 & 9.8100 \mathrm{E}-03 \\ \mathrm{SS}-316 & 1.8990 \mathrm{E}-02 & 1.8990 \mathrm{E}-02\end{array}$

$\begin{array}{lll}10 & 46.14 & 62.78\end{array}$

$\begin{array}{lll}\mathrm{U}-235 & 1.4143 \mathrm{E}-05 & 1.3721 \mathrm{E}-05 \\ \mathrm{U}-238 & 7.0573 \mathrm{E}-03 & 7.0350 \mathrm{E}-03 \\ \mathrm{Pu}-239 & 0.0000 \mathrm{E}+00 & 2.0506 \mathrm{E}-05 \\ \mathrm{PU}-240 & 0.0000 \mathrm{E}+00 & 8.3485 \mathrm{E}-08 \\ \mathrm{FP} & 0.0000 \mathrm{E}+00 & 2.0017 \mathrm{E}-06 \\ 0-16 & 1.4143 \mathrm{E}-02 & 1.4143 \mathrm{E}-02 \\ \mathrm{Na}-23 & 9.7416 \mathrm{E}-03 & 9.7416 \mathrm{E}-03 \\ \mathrm{SS}-316 & 1.9073 \mathrm{E}-02 & 1.9073 \mathrm{E}-02\end{array}$

$11 \quad 62.78 \quad 116.00$

$\begin{array}{lll}\mathrm{Na}-23 & 8.2400 \mathrm{E}-03 & 8.2400 \mathrm{E}-03 \\ \mathrm{SS}-316 & 2.5562 \mathrm{E}-02 & 2.5562 \mathrm{E}-02\end{array}$


Table B10A-3.1. Fission Power Generated in MFA-1, MFA-2 and Neighboring Assemblies at BOC 10A-3

\begin{tabular}{lccccc} 
CORE & $* * * * * *$ & \multicolumn{2}{c}{ POWER IN MEGAWATTS } & $* * * * * * *$ \\
POS. & BELOW CORE & \multicolumn{2}{c}{ IN CORE } & ABOVE CORE & TOTAL PWR \\
1506 & $2.923 \mathrm{E}-02$ & $3.888 \mathrm{E}+00$ & $2.016 \mathrm{E}-02$ & $3.937 \mathrm{E}+00$ \\
1404 & $8.347 \mathrm{E}-02$ & $4.6515 \mathrm{E}+00$ & $4.578 \mathrm{E}-02$ & $4.785 \mathrm{E}+00$ \\
1405 & $9.573 \mathrm{E}-03$ & $3.886 \mathrm{E}+00$ & $7.157 \mathrm{E}-03$ & $3.903 \mathrm{E}+00$ \\
1505 & $1.134 \mathrm{E}-02$ & $3.560 \mathrm{E}+00$ & $6.692 \mathrm{E}-03$ & $3.578 \mathrm{E}+00$ \\
1507 & $9.828 \mathrm{E}-03$ & $3.290 \mathrm{E}+00$ & $7.354 \mathrm{E}-03$ & $3.307 \mathrm{E}+00$ \\
1606 & $6.233 \mathrm{E}-03$ & $3.462 \mathrm{E}+00$ & $4.559 \mathrm{E}-03$ & $3.473 \mathrm{E}+00$ \\
1607 & $9.179 \mathrm{E}-03$ & $2.857 \mathrm{E}+00$ & $6.639 \mathrm{E}-03$ & $2.872 \mathrm{E}+00$ \\
2507 & $3.000 \mathrm{E}-02$ & $4.145 \mathrm{E}+00$ & $2.203 \mathrm{E}-02$ & $4.197 \mathrm{E}+00$ \\
2405 & $7.196 \mathrm{E}-02$ & $4.423 \mathrm{E}+00$ & $4.182 \mathrm{E}-02$ & $4.537 \mathrm{E}+00$ \\
2506 & $1.144 \mathrm{E}-02$ & $3.850 \mathrm{E}+00$ & $8.082 \mathrm{E}-03$ & $3.870 \mathrm{E}+00$ \\
2508 & $1.108 \mathrm{E}-02$ & $4.557 \mathrm{E}+00$ & $8.140 \mathrm{E}-03$ & $4.576 \mathrm{E}+00$ \\
2607 & $7.842 \mathrm{E}-03$ & $3.538 \mathrm{E}+00$ & $5.861 \mathrm{E}-03$ & $3.551 \mathrm{E}+00$ \\
2608 & $4.991 \mathrm{E}-03$ & $3.398 \mathrm{E}+00$ & $4.147 \mathrm{E}-03$ & $3.408 \mathrm{E}+00$ \\
2609 & $9.444 \mathrm{E}-02$ & $3.150 \mathrm{E}+00$ & $5.240 \mathrm{E}-02$ & $3.297 \mathrm{E}+00$
\end{tabular}

Table B10A-3.2. Assembly Averaged Total Fiux for MFA-1 and MFA-2 at BOC $10 \mathrm{~A}-3$

$\begin{array}{ccc} & \text { Core } & \text { Total FTux } \\ \text { Assembly } & \text { Pos. } & \left(\mathrm{n} / \mathrm{cm}^{2}-\mathrm{sec}\right) \\ \text { MFA-1 } & 1506 & 2.874 E+15 \\ \text { MFA-2 } & 2507 & 3.059 E+15\end{array}$


Table B10A-3.3. Axial Distribution of Total Flux, Fast Flux and Power in MFA-1 at BOC 10A-3

$\begin{array}{rccc}z(\mathrm{~cm}) & \text { Total Flux } & \text { Flux }>0.1 \mathrm{MeV} & \text { Power } \\ -97.78 & 2.7544 \mathrm{E}-02 & 1.3954 \mathrm{E}-02 & 0.0000 \mathrm{E}+00 \\ -92.78 & 5.9206 \mathrm{E}-02 & 2.6947 \mathrm{E}-02 & 0.0000 \mathrm{E}+00 \\ -87.78 & 9.4932 \mathrm{E}-02 & 4.3121 \mathrm{E}-02 & 0.0000 \mathrm{E}+00 \\ -82.78 & 1.3742 \mathrm{E}-01 & 6.4325 \mathrm{E}-02 & 0.0000 \mathrm{E}+00 \\ -77.78 & 1.8939 \mathrm{E}-01 & 9.2832 \mathrm{E}-02 & 0.0000 \mathrm{E}+00 \\ -72.15 & 2.4815 \mathrm{E}-01 & 1.2970 \mathrm{E}-01 & 0.0000 \mathrm{E}+00 \\ -65.90 & 3.0652 \mathrm{E}-01 & 1.7598 \mathrm{E}-01 & 0.0000 \mathrm{E}+00 \\ -60.35 & 3.6801 \mathrm{E}-01 & 2.3819 \mathrm{E}-01 & 2.7593 \mathrm{E}-02 \\ -55.49 & 4.4981 \mathrm{E}-01 & 3.2261 \mathrm{E}-01 & 3.6261 \mathrm{E}-02 \\ -50.62 & 5.4546 \mathrm{E}-01 & 4.3347 \mathrm{E}-01 & 5.0392 \mathrm{E}-02 \\ -47.17 & 6.2291 \mathrm{E}-01 & 5.3906 \mathrm{E}-01 & 6.7363 \mathrm{E}-02 \\ -43.58 & 7.0456 \mathrm{E}-01 & 6.5929 \mathrm{E}-01 & 7.4547 \mathrm{E}-01 \\ -38.45 & 8.2159 \mathrm{E}-01 & 8.0352 \mathrm{E}-01 & 8.3617 \mathrm{E}-01 \\ -33.32 & 9.2971 \mathrm{E}-01 & 9.2582 \mathrm{E}-01 & 9.3597 \mathrm{E}-01 \\ -28.20 & 1.0239 \mathrm{E}+00 & 1.0272 \mathrm{E}+00 & 1.0192 \mathrm{E}+00 \\ -23.07 & 1.102 .2 \mathrm{E}+00 & 1.1093 \mathrm{E}+00 & 1.0954 \mathrm{E}+00 \\ -17.94 & 1.1631 \mathrm{E}+00 & 1.1722 \mathrm{E}+00 & 1.1551 \mathrm{E}+00 \\ -12.82 & 1.2057 \mathrm{E}+00 & 1.2154 \mathrm{E}+00 & 1.1923 \mathrm{E}+00 \\ -7.69 & 1.2298 \mathrm{E}+00 & 1.2399 \mathrm{E}+00 & 1.2159 \mathrm{E}+00 \\ -2.56 & 1.2353 \mathrm{E}+00 & 1.2456 \mathrm{E}+00 & 1.2211 \mathrm{E}+00 \\ 2.56 & 1.222 .1 \mathrm{E}+00 & 1.2327 \mathrm{E}+00 & 1.2094 \mathrm{E}+00 \\ 7.69 & 1.1905 \mathrm{E}+00 & 1.2012 \mathrm{E}+00 & 1.1780 \mathrm{E}+00 \\ 12.82 & 1.1413 \mathrm{E}+00 & 1.1520 \mathrm{E}+00 & 1.1294 \mathrm{E}+00 \\ 17.94 & 1.0759 \mathrm{E}+00 & 1.0865 \mathrm{E}+00 & 1.0712 \mathrm{E}+00 \\ 23.07 & 9.9554 \mathrm{E}-01 & 1.0048 \mathrm{E}+00 & 9.9164 \mathrm{E}-01 \\ 28.20 & 9.0235 \mathrm{E}-01 & 9.0856 \mathrm{E}-01 & 8.9985 \mathrm{E}-01 \\ 33.32 & 7.9865 \mathrm{E}-01 & 7.9927 \mathrm{E}-01 & 8.0564 \mathrm{E}-01 \\ 38.45 & 6.8679 \mathrm{E}-01 & 6.7644 \mathrm{E}-01 & 6.9875 \mathrm{E}-01 \\ 43.58 & 5.7114 \mathrm{E}-01 & 5.4028 \mathrm{E}-01 & 5.9942 \mathrm{E}-01 \\ 47.17 & 4.9233 \mathrm{E}-01 & 4.3346 \mathrm{E}-01 & 5.1245 \mathrm{E}-02 \\ 50.62 & 4.1793 \mathrm{E}-01 & 3.3987 \mathrm{E}-01 & 3.6473 \mathrm{E}-02 \\ 55.49 & 3.2644 \mathrm{E}-01 & 2.4205 \mathrm{E}-01 & 2.4051 \mathrm{E}-02 \\ 60.35 & 2.4746 \mathrm{E}-01 & 1.6758 \mathrm{E}-01 & 1.6265 \mathrm{E}-02 \\ 64.98 & 1.9916 \mathrm{E}-01 & 1.2529 \mathrm{E}-01 & 0.0000 \mathrm{E}+00 \\ 69.39 & 1.7102 \mathrm{E}-01 & 1.0314 \mathrm{E}-01 & 0.0000 \mathrm{E}+00 \\ 76.46 & 1.3076 \mathrm{E}-01 & 7.3464 \mathrm{E}-02 & 0.0000 \mathrm{E}+00 \\ 86.80 & 8.5815 \mathrm{E}-02 & 4.4486 \mathrm{E}-02 & 0.0000 \mathrm{E}+00 \\ 98.14 & 4.9740 \mathrm{E}-02 & 2.4398 \mathrm{E}-02 & 0.0000 \mathrm{E}+00 \\ 110.00 & 2.1379 \mathrm{E}-02 & 1.0970 \mathrm{E}-02 & 0.0000 \mathrm{E}+00\end{array}$


Table B10A-3.4. Axial Distribution of Total Flux, Fast Flux and Power in MFA-2 at $B O C$ 1OA-3

$\begin{array}{rccc}z(\mathrm{~cm}) & \text { Total Flux } & \text { Flux }>0.1 \mathrm{MeV} & \text { Power } \\ -97.78 & 2.5372 \mathrm{E}-02 & 1.2956 \mathrm{E}-02 & 0.0000 \mathrm{E}+00 \\ -92.78 & 5.4484 \mathrm{E}-02 & 2.5004 \mathrm{E}-02 & 0.0000 \mathrm{E}+00 \\ -87.78 & 8.7356 \mathrm{E}-02 & 4.0013 \mathrm{E}-02 & 0.0000 \mathrm{E}+00 \\ -82.78 & 1.26 ! 56 \mathrm{E}-01 & 5.9739 \mathrm{E}-02 & 0.0000 \mathrm{E}+00 \\ -77.78 & 1.7484 \mathrm{E}-01 & 8.6392 \mathrm{E}-02 & 0.0000 \mathrm{E}+00 \\ -72.15 & 2.3013 \mathrm{E}-01 & 1.2117 \mathrm{E}-01 & 0.0000 \mathrm{E}+00 \\ -65.90 & 2.8687 \mathrm{E}-01 & 1.6591 \mathrm{E}-01 & 0.0000 \mathrm{E}+00 \\ -60.35 & 3.4830 \mathrm{E}-01 & 2.2703 \mathrm{E}-01 & 2.5974 \mathrm{E}-02 \\ -55.49 & 4.2994 \mathrm{E}-01 & 3.1008 \mathrm{E}-01 & 3.4715 \mathrm{E}-02 \\ -50.62 & 5.2644 \mathrm{E}-01 & 4.1975 \mathrm{E}-01 & 4.8897 \mathrm{E}-02 \\ -47.17 & 6.0532 \mathrm{E}-01 & 5.2466 \mathrm{E}-01 & 6.5829 \mathrm{E}-02 \\ -43.58 & 6.8870 \mathrm{E}-01 & 6.4452 \mathrm{E}-01 & 7.2770 \mathrm{E}-01 \\ -38.45 & 8.0800 \mathrm{E}-01 & 7.9004 \mathrm{E}-01 & 8.2223 \mathrm{E}-01 \\ -33.32 & 9.1834 \mathrm{E}-01 & 9.1440 \mathrm{E}-01 & 9.2450 \mathrm{E}-01 \\ -28.20 & 1.0147 \mathrm{E}+00 & 1.0181 \mathrm{E}+00 & 1.0100 \mathrm{E}+00 \\ -23.07 & 1.0954 \mathrm{E}+00 & 1.1029 \mathrm{E}+00 & 1.0885 \mathrm{E}+00 \\ -17.94 & 1.1588 \mathrm{E}+00 & 1.1683 \mathrm{E}+00 & 1.1507 \mathrm{E}+00 \\ -12.82 & 1.2039 \mathrm{E}+00 & 1.2139 \mathrm{E}+00 & 1.1905 \mathrm{E}+00 \\ -7.69 & 1.2301 \mathrm{E}+00 & 1.2405 \mathrm{E}+00 & 1.2163 \mathrm{E}+00 \\ -2.56 & 1.2372 \mathrm{E}+00 & 1.2478 \mathrm{E}+00 & 1.2232 \mathrm{E}+00 \\ 2.56 & 1.2254 \mathrm{E}+00 & 1.2363 \mathrm{E}+00 & 1.2126 \mathrm{E}+00 \\ 7.69 & 1.1951 \mathrm{E}+00 & 1.2061 \mathrm{E}+00 & 1.1827 \mathrm{E}+00 \\ 12.82 & 1.1475 \mathrm{E}+00 & 1.1584 \mathrm{E}+00 & 1.1357 \mathrm{E}+00 \\ 17.94 & 1.0836 \mathrm{E}+00 & 1.0941 \mathrm{E}+00 & 1.0789 \mathrm{E}+00 \\ 23.07 & 1.004 .3 \mathrm{E}+00 & 1.0133 \mathrm{E}+00 & 1.0007 \mathrm{E}+00 \\ 28.20 & 9.1152 \mathrm{E}-01 & 9.1720 \mathrm{E}-01 & 9.0934 \mathrm{E}-01 \\ 33.32 & 8.0726 \mathrm{E}-01 & 8.0719 \mathrm{E}-01 & 8.1464 \mathrm{E}-01 \\ 38.45 & 6.9394 \mathrm{E}-01 & 6.8268 \mathrm{E}-01 & 7.0643 \mathrm{E}-01 \\ 43.58 & 5.7621 \mathrm{E}-01 & 5.4432 \mathrm{E}-01 & 6.0535 \mathrm{E}-01 \\ 47.17 & 4.9585 \mathrm{E}-01 & 4.3595 \mathrm{E}-01 & 5.2402 \mathrm{E}-02 \\ 50.62 & 4.2020 \mathrm{E}-01 & 3.4131 \mathrm{E}-01 & 3.7358 \mathrm{E}-02 \\ 55.49 & 3.2791 \mathrm{E}-01 & 2.4286 \mathrm{E}-01 & 2.4688 \mathrm{E}-02 \\ 60.35 & 2.4890 \mathrm{E}-01 & 1.6825 \mathrm{E}-01 & 1.6738 \mathrm{E}-02 \\ 64.98 & 2.0052 \mathrm{E}-01 & 1.2563 \mathrm{E}-01 & 0.0000 \mathrm{E}+00 \\ 69.39 & 1.7179 \mathrm{E}-01 & 1.0303 \mathrm{E}-01 & 0.0000 \mathrm{E}+00 \\ 76.46 & 1.3062 \mathrm{E}-01 & 7.2781 \mathrm{E}-02 & 0.0000 \mathrm{E}+00 \\ 86.80 & 8.5140 \mathrm{E}-02 & 4.3610 \mathrm{E}-02 & 0.0000 \mathrm{E}+00 \\ 98.14 & 4.8997 \mathrm{E}-02 & 2.3654 \mathrm{E}-02 & 0.0000 \mathrm{E}+00 \\ 110.00 & 2.0763 \mathrm{E}-02 & 1.0461 \mathrm{E}-02 & 0.0000 \mathrm{E}+00 \\ & & & \end{array}$


Table B10A-3.5. Fission Power Distribution by Pin in MFA-1 at BOC 10A-3

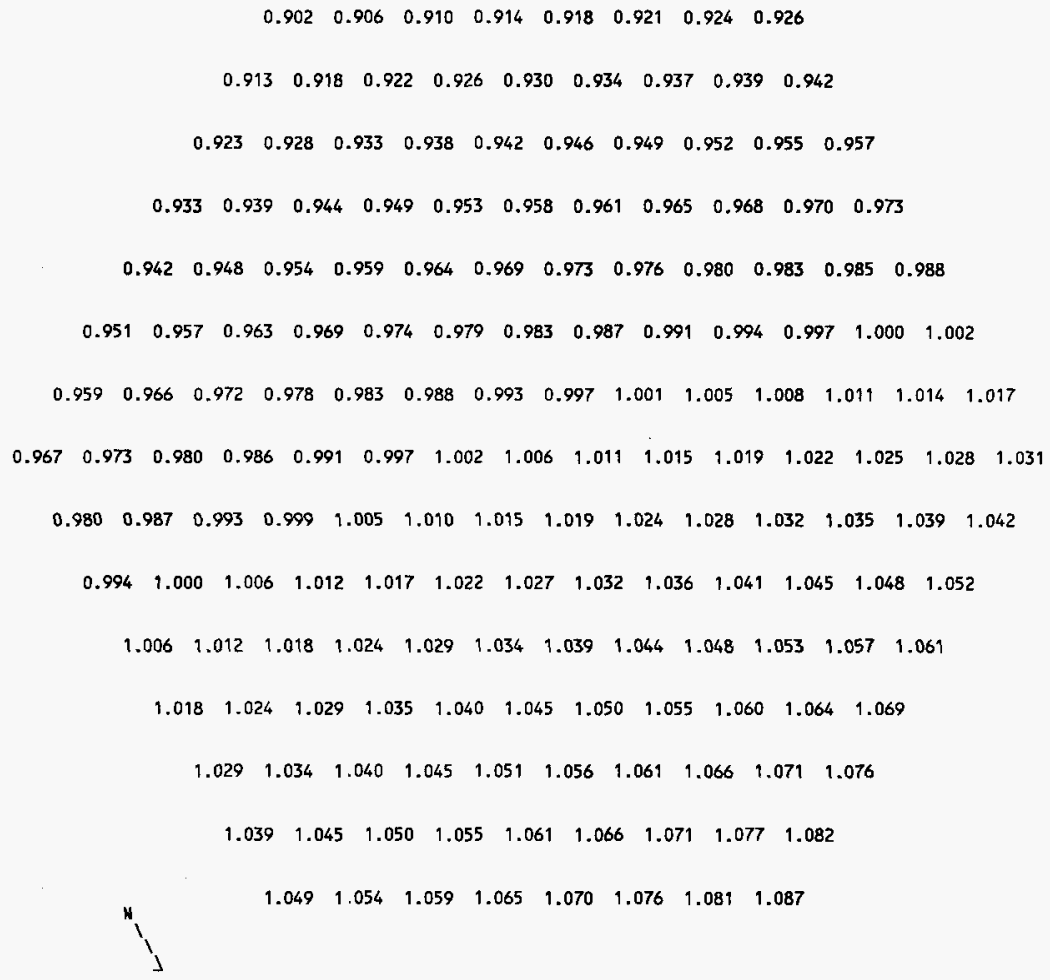




\section{WHC-SD-FF-ANAL-009 REV 0}

Table B10A-3.6. Fission Power Distribution by Pin in MFA-2 at BOC $10 A-3$

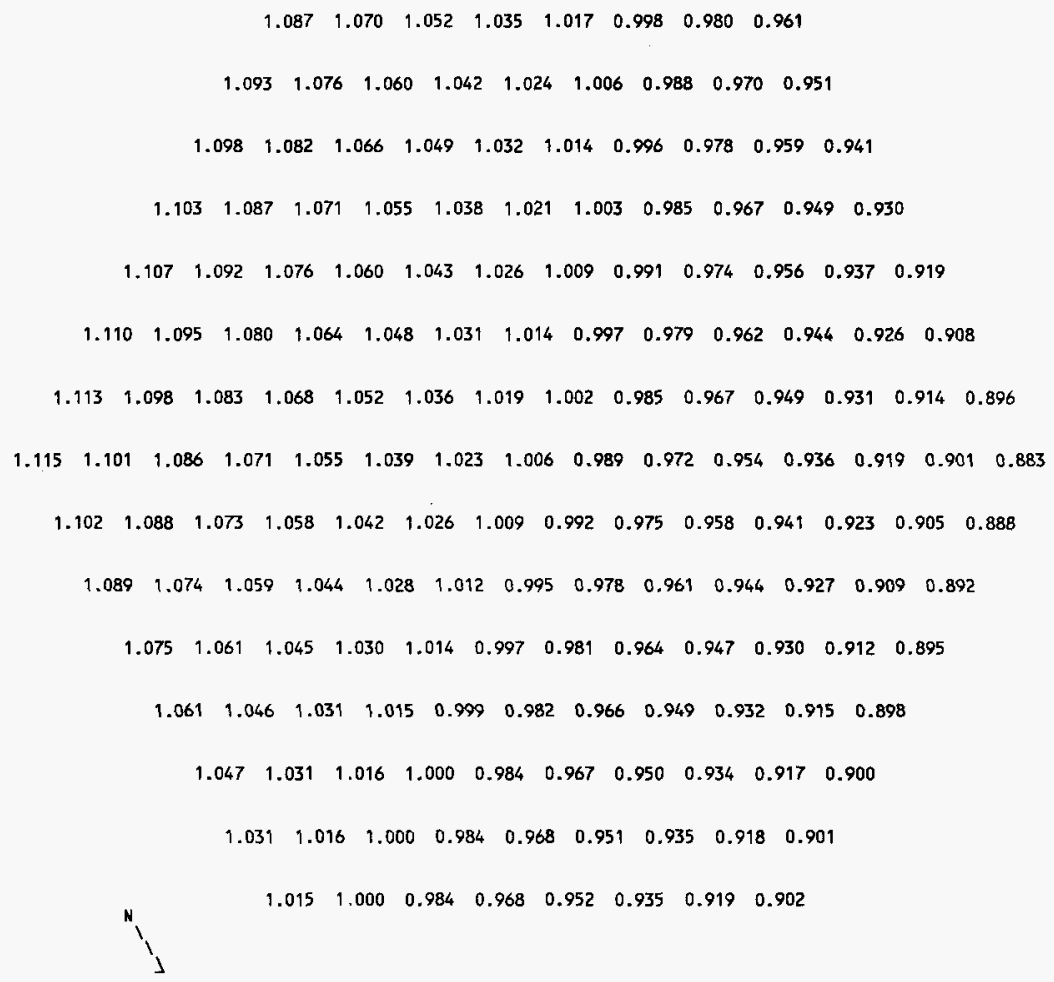


Table B10A-3.7. Total Flux Distribution by Pin in MFA-1 at BOC 10A-3

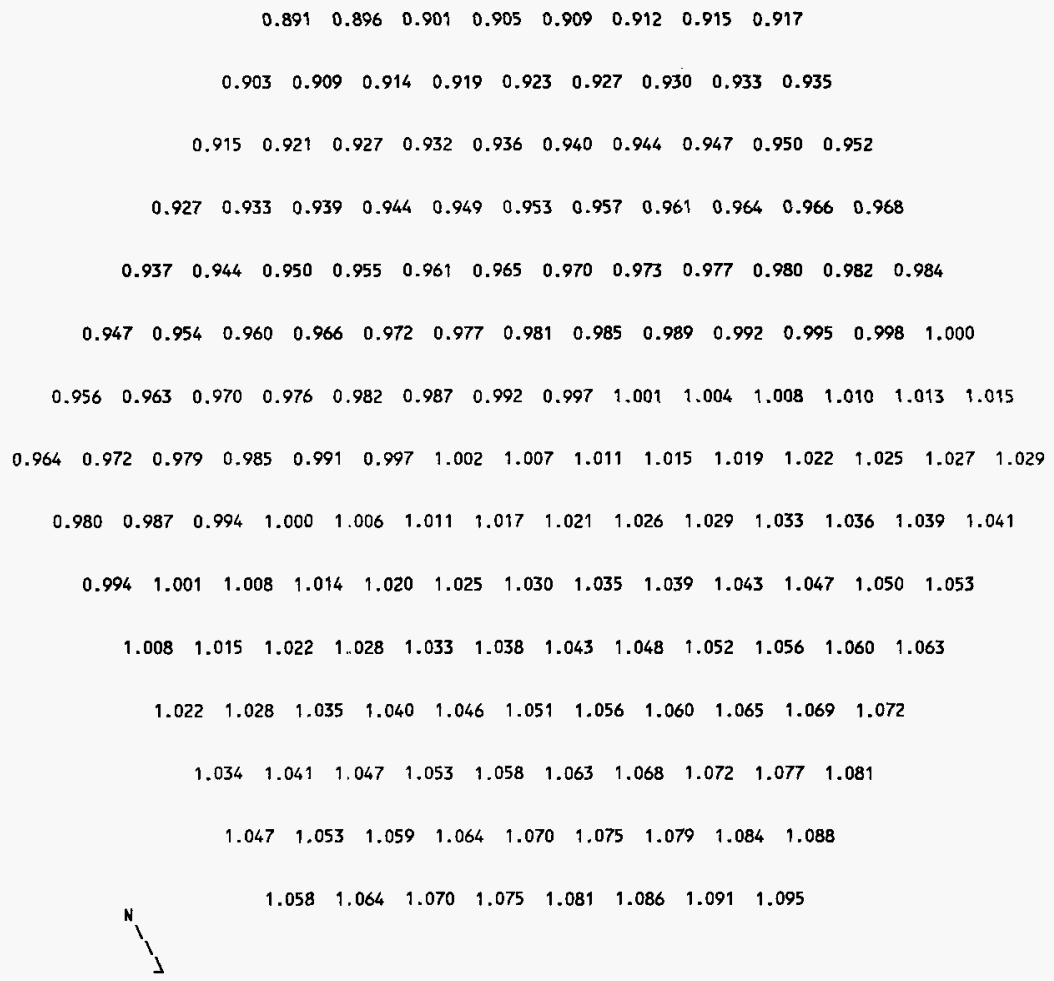




\section{WHC-SD-FF-ANAL-009 REV 0}

Table B10A-3.8. Total Flux Distribution by Pin in MFA-2 at BOC 10A-3

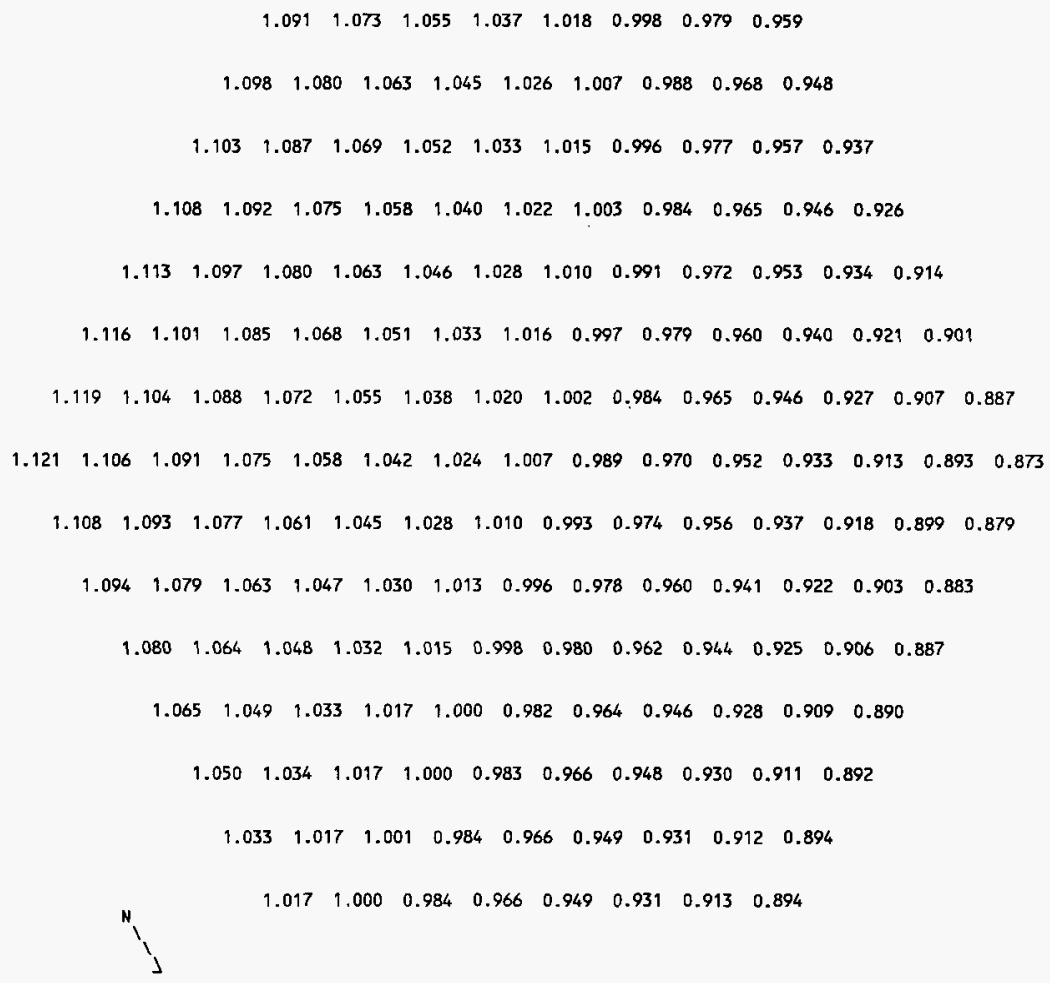


Table B10A-3.9. MFA-1 and MFA-2 Duct Wall Flux Greater Than $0.1 \mathrm{MeV}$ at BOC 10A-3

$\begin{array}{ccc}\text { SIDE } & \begin{array}{c}\text { FLUX }>0.1 \\ \text { MFA-1 }\end{array} & \begin{array}{c}\text { MEV } \\ \left(1 \mathrm{n} / \mathrm{cm}^{2}-\mathrm{sec}\right) \\ \text { MFA-2 }\end{array} \\ \text { E } & 1.8787 \mathrm{E}+15 & 2.1340 \mathrm{E}+15 \\ \text { SE } & 1.6787 \mathrm{E}+15 & 2.2202 \mathrm{E}+15 \\ \text { SW } & 1.6099 \mathrm{E}+15 & 2.0130 \mathrm{E}+15 \\ \text { W } & 1.7705 \mathrm{E}+15 & 1.7564 \mathrm{E}+15 \\ \text { NW } & 1.9888 \mathrm{E}+15 & 1.6795 \mathrm{E}+15 \\ \text { NE } & 2.0449 \mathrm{E}+15 & 1.8375 \mathrm{E}+15\end{array}$

Table B10A-3.10. Assembly Outlet Temperatures and Flow Rates at $\mathrm{BOC}$ 10A-3

\section{MEASURED}

CORE OUTLET TEMP. FLOW RATE

POS. (DEG F) (LB/ $/ \mathrm{H}$ )

$\begin{array}{lll}1506 & 1033 & 114940\end{array}$

$1404 \quad 916 \quad 225440$

$1405 \quad 890 \quad 208130$

$\begin{array}{lll}1505 & 884 & 208130\end{array}$

$1507 \quad 873 \quad 208130$

$1606 \quad 902 \quad 170680$

$\begin{array}{lll}1607 & 871 & 170680\end{array}$

$2507 \quad 1058 \quad 114940$

$2405 \quad 945 \quad 192410$

$2506 \quad 900 \quad 197920$

$2508 \quad 927 \quad 208130$

$2607 \quad 922 \quad 163690$

$2608 \quad 899 \quad 170680$

$\begin{array}{lll}2609 & 897 & 173340\end{array}$ 
Table E10A-3.1. Fission Power Generated in MFA-1, MFA-2 and Neighboring Assemblies at EOC 10A-3

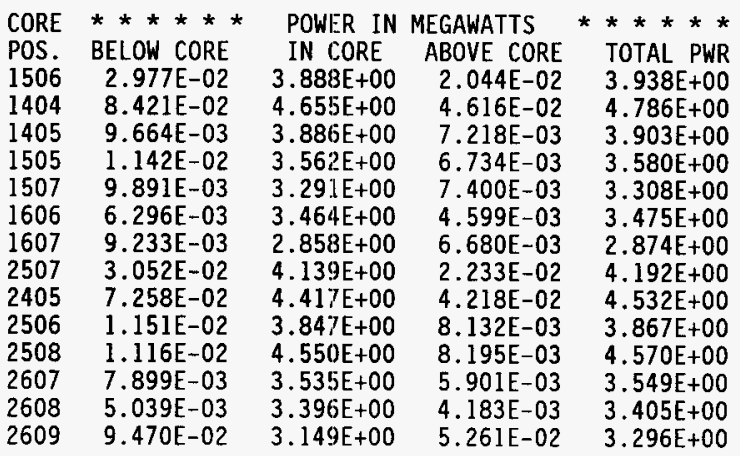

Table E10A-3.2. Assembly Averaged Total Flux for MFA-1 and MFA-2 at EOC 10A-3

$\begin{array}{ccc} & \text { Core } & \text { Total Flux } \\ \text { Assembly } & \text { Pos. } & \left(\mathrm{n} / \mathrm{cm}^{2}-\mathrm{sec}\right) \\ \text { MFA-1 } & 1506 & 2.879 \mathrm{E}+15 \\ \text { MFA-2 } & 2507 & 3.061 \mathrm{E}+15\end{array}$


Table ElOA-3.3. Axial Distribution of Total Flux, Fast Flux and Power in MFA-1 at EOC 10A-3

$\begin{array}{rccc}\text { Z (cm) } & \text { Total Flux } & \text { Flux > 0.1 MeV } & \text { Power } \\ -97.78 & 2.7554 \mathrm{E}-02 & 1.3965 \mathrm{E}-02 & 0.0000 \mathrm{E}+00 \\ -92.78 & 5.9223 \mathrm{E}-02 & 2.6967 \mathrm{E}-02 & 0.0000 \mathrm{E}+00 \\ -87.78 & 9.4960 \mathrm{E}-02 & 4.3154 \mathrm{E}-02 & 0.0000 \mathrm{E}+00 \\ -82.78 & 1.3746 \mathrm{E}-01 & 6.4373 \mathrm{E}-02 & 0.0000 \mathrm{E}+00 \\ -77.78 & 1.8944 \mathrm{E}-01 & 9.2904 \mathrm{E}-02 & 0.0000 \mathrm{E}+00 \\ -72.15 & 2.4821 \mathrm{E}-01 & 1.2979 \mathrm{E}-01 & 0.0000 \mathrm{E}+00 \\ -65.90 & 3.0659 \mathrm{E}-01 & 1.7611 \mathrm{E}-01 & 0.0000 \mathrm{E}+00 \\ -60.35 & 3.6807 \mathrm{E}-01 & 2.3838 \mathrm{E}-01 & 2.8295 \mathrm{E}-02 \\ -55.49 & 4.4987 \mathrm{E}-01 & 3.2284 \mathrm{E}-01 & 3.7025 \mathrm{E}-02 \\ -50.62 & 5.4555 \mathrm{E}-01 & 4.3370 \mathrm{E}-01 & 5.1212 \mathrm{E}-02 \\ -47.17 & 6.2299 \mathrm{E}-01 & 5.3924 \mathrm{E}-01 & 6.8217 \mathrm{E}-02 \\ -43.58 & 7.0466 \mathrm{E}-01 & 6.5942 \mathrm{E}-01 & 7.4576 \mathrm{E}-01 \\ -38.45 & 8.21616 \mathrm{E}-01 & 8.0362 \mathrm{E}-01 & 8.3650 \mathrm{E}-01 \\ -33.32 & 9.2971 \mathrm{E}-01 & 9.2586 \mathrm{E}-01 & 9.3630 \mathrm{E}-01 \\ -28.20 & 1.0258 \mathrm{E}+00 & 1.0272 \mathrm{E}+00 & 1.0191 \mathrm{E}+00 \\ -23.07 & 1.1020 \mathrm{E}+00 & 1.1092 \mathrm{E}+00 & 1.0952 \mathrm{E}+00 \\ -17.94 & 1.1629 \mathrm{E}+00 & 1.1720 \mathrm{E}+00 & 1.1548 \mathrm{E}+00 \\ -12.82 & 1.2054 \mathrm{E}+00 & 1.2152 \mathrm{E}+00 & 1.1917 \mathrm{E}+00 \\ -7.69 & 1.2296 \mathrm{E}+00 & 1.2396 \mathrm{E}+00 & 1.2152 \mathrm{E}+00 \\ -2.56 & 1.2350 \mathrm{E}+00 & 1.2453 \mathrm{E}+00 & 1.2205 \mathrm{E}+00 \\ 2.56 & 1.2219 \mathrm{E}+00 & 1.2324 \mathrm{E}+00 & 1.2089 \mathrm{E}+00 \\ 7.69 & 1.1904 \mathrm{E}+00 & 1.2010 \mathrm{E}+00 & 1.1776 \mathrm{E}+00 \\ 12.82 & 1.1413 \mathrm{E}+00 & 1.1520 \mathrm{E}+00 & 1.1291 \mathrm{E}+00 \\ 17.94 & 1.0760 \mathrm{E}+00 & 1.0865 \mathrm{E}+00 & 1.0713 \mathrm{E}+00 \\ 23.07 & 9.9570 \mathrm{E}-01 & 1.0050 \mathrm{E}+00 & 9.9189 \mathrm{E}-01 \\ 28.20 & 9.0255 \mathrm{E}-01 & 9.0879 \mathrm{E}-01 & 9.0015 \mathrm{E}-01 \\ 33.32 & 7.9890 \mathrm{E}-01 & 7.9959 \mathrm{E}-01 & 8.0644 \mathrm{E}-01 \\ 38.45 & 6.8706 \mathrm{E}-01 & 6.7681 \mathrm{E}-01 & 6.9948 \mathrm{E}-01 \\ 43.58 & 5.7142 \mathrm{E}-01 & 5.4062 \mathrm{E}-01 & 6.0006 \mathrm{E}-01 \\ 47.17 & 4.9255 \mathrm{E}-01 & 4.3377 \mathrm{E}-01 & 5.1757 \mathrm{E}-02 \\ 50.62 & 4.1813 \mathrm{E}-01 & 3.4015 \mathrm{E}-01 & 3.6930 \mathrm{E}-02 \\ 55.49 & 3.2662 \mathrm{E}-01 & 2.4227 \mathrm{E}-01 & 2.4440 \mathrm{E}-02 \\ 60.35 & 2.4760 \mathrm{E}-01 & 1.6775 \mathrm{E}-01 & 1.6587 \mathrm{E}-02 \\ 64.98 & 1.9928 \mathrm{E}-01 & 1.2542 \mathrm{E}-01 & 0.0000 \mathrm{E}+00 \\ 69.39 & 1.7112 \mathrm{E}-01 & 1.0325 \mathrm{E}-01 & 0.0000 \mathrm{E}+00 \\ 76.46 & 1.3084 \mathrm{E}-01 & 7.3539 \mathrm{E}-02 & 0.0000 \mathrm{E}+00 \\ 86.80 & 8.5871 \mathrm{E}-02 & 4.4530 \mathrm{E}-02 & 0.0000 \mathrm{E}+00 \\ 98.14 & 4.9771 \mathrm{E}-02 & 2.4422 \mathrm{E}-02 & 0.0000 \mathrm{E}+00 \\ 110.00 & 2.1393 \mathrm{E}-02 & 1.0982 \mathrm{E}-02 & 0.0000 \mathrm{E}+00 \\ & & & \end{array}$


Table ElOA-3.4. Axial Distribution of Total Flux, Fast Flux and Power in MFA-2 at EOC $10 \mathrm{~A}-3$

$\begin{array}{rccc}z(\mathrm{~cm}) & \text { Total Flux } & \text { Flux }>0.1 \text { MeV } & \text { Power } \\ -97.78 & 2.5391 \mathrm{E}-02 & 1.2971 \mathrm{E}-02 & 0.0000 \mathrm{E}+00 \\ -92.78 & 5.4521 \mathrm{E}-02 & 2.5032 \mathrm{E}-02 & 0.0000 \mathrm{E}+00 \\ -87.78 & 8.7413 \mathrm{E}-02 & 4.0057 \mathrm{E}-02 & 0.0000 \mathrm{E}+00 \\ -82.78 & 1.2664 \mathrm{E}-01 & 5.9801 \mathrm{E}-02 & 0.0000 \mathrm{E}+00 \\ -77.78 & 1.7495 \mathrm{E}-01 & 8.6484 \mathrm{E}-02 & 0.0000 \mathrm{E}+00 \\ -72.15 & 2.3025 \mathrm{E}-01 & 1.2129 \mathrm{E}-01 & 0.0000 \mathrm{E}+00 \\ -65.90 & 2.8700 \mathrm{E}-01 & 1.6607 \mathrm{E}-01 & 0.0000 \mathrm{E}+00 \\ -60.35 & 3.4847 \mathrm{E}-01 & 2.2725 \mathrm{E}-01 & 2.6648 \mathrm{E}-02 \\ -55.49 & 4.3011 \mathrm{E}-01 & 3.1034 \mathrm{E}-01 & 3.5453 \mathrm{E}-02 \\ -50.62 & 5.2660 \mathrm{E}-01 & 4.2001 \mathrm{E}-01 & 4.9700 \mathrm{E}-02 \\ -47.17 & 6.0547 \mathrm{E}-01 & 5.2486 \mathrm{E}-01 & 6.6673 \mathrm{E}-02 \\ -43.58 & 6.8881 \mathrm{E}-01 & 6.4471 \mathrm{E}-01 & 7.2809 \mathrm{E}-01 \\ -38.45 & 8.0812 \mathrm{E}-01 & 7.9020 \mathrm{E}-01 & 8.2266 \mathrm{E}-01 \\ -33.32 & 9.1836 \mathrm{E}-01 & 9.1444 \mathrm{E}-01 & 9.2492 \mathrm{E}-01 \\ -28.20 & 1.0147 \mathrm{E}+00 & 1.0181 \mathrm{E}+00 & 1.0098 \mathrm{E}+00 \\ -23.07 & 1.0952 \mathrm{E}+00 & 1.1027 \mathrm{E}+00 & 1.0883 \mathrm{E}+00 \\ -17.94 & 1.1586 \mathrm{E}+00 & 1.1680 \mathrm{E}+00 & 1.1504 \mathrm{E}+00 \\ -12.82 & 1.2036 \mathrm{E}+00 & 1.2136 \mathrm{E}+00 & 1.1899 \mathrm{E}+00 \\ -7.69 & 1.2298 \mathrm{E}+00 & 1.2401 \mathrm{E}+00 & 1.2155 \mathrm{E}+00 \\ -2.56 & 1.2369 \mathrm{E}+00 & 1.2475 \mathrm{E}+00 & 1.2224 \mathrm{E}+00 \\ 2.56 & 1.2251 \mathrm{E}+00 & 1.2360 \mathrm{E}+00 & 1.2120 \mathrm{E}+00 \\ 7.69 & 1.1950 \mathrm{E}+00 & 1.2059 \mathrm{E}+00 & 1.1822 \mathrm{E}+00 \\ 12.82 & 1.1474 \mathrm{E}+00 & 1.1583 \mathrm{E}+00 & 1.1353 \mathrm{E}+00 \\ 17.94 & 1.0837 \mathrm{E}+00 & 1.0941 \mathrm{E}+00 & 1.0791 \mathrm{E}+00 \\ 23.07 & 1.0045 \mathrm{E}+00 & 1.0134 \mathrm{E}+00 & 1.0009 \mathrm{E}+00 \\ 28.20 & 9.1174 \mathrm{E}-01 & 9.1749 \mathrm{E}-01 & 9.0966 \mathrm{E}-01 \\ 33.32 & 8.0757 \mathrm{E}-01 & 8.0759 \mathrm{E}-01 & 8.1550 \mathrm{E}-01 \\ 38.45 & 6.9431 \mathrm{E}-01 & 6.8315 \mathrm{E}-01 & 7.0727 \mathrm{E}-01 \\ 43.58 & 5.7654 \mathrm{E}-01 & 5.4471 \mathrm{E}-01 & 6.0609 \mathrm{E}-01 \\ 47.17 & 4.9616 \mathrm{E}-01 & 4.3632 \mathrm{E}-01 & 5.2957 \mathrm{E}-02 \\ 50.62 & 4.2047 \mathrm{E}-01 & 3.4164 \mathrm{E}-01 & 3.7859 \mathrm{E}-02 \\ 55.49 & 3.2816 \mathrm{E}-01 & 2.4313 \mathrm{E}-01 & 2.5112 \mathrm{E}-02 \\ 60.35 & 2.4910 \mathrm{E}-01 & 1.6846 \mathrm{E}-01 & 1.7092 \mathrm{E}-02 \\ 64.98 & 2.0068 \mathrm{E}-01 & 1.2579 \mathrm{E}-01 & 0.0000 \mathrm{E}+00 \\ 69.39 & 1.7195 \mathrm{E}-01 & 1.0315 \mathrm{E}-01 & 0.0000 \mathrm{E}+00 \\ 76.46 & 1.3075 \mathrm{E}-01 & 7.2875 \mathrm{E}-02 & 0.0000 \mathrm{E}+00 \\ 86.80 & 8.5228 \mathrm{E}-02 & 4.3670 \mathrm{E}-02 & 0.0000 \mathrm{E}+00 \\ 98.14 & 4.9050 \mathrm{E}-02 & 2.3688 \mathrm{E}-02 & 0.0000 \mathrm{E}+00 \\ 110.00 & 2.0786 \mathrm{E}-02 & 1.0476 \mathrm{E}-02 & 0.0000 \mathrm{E}+00\end{array}$




\section{WHC-SD-FF-ANAL-009 REV 0}

Table E1OA-3.5. Fission Power Distribution by Pin in MFA-1 at EOC 10A-3

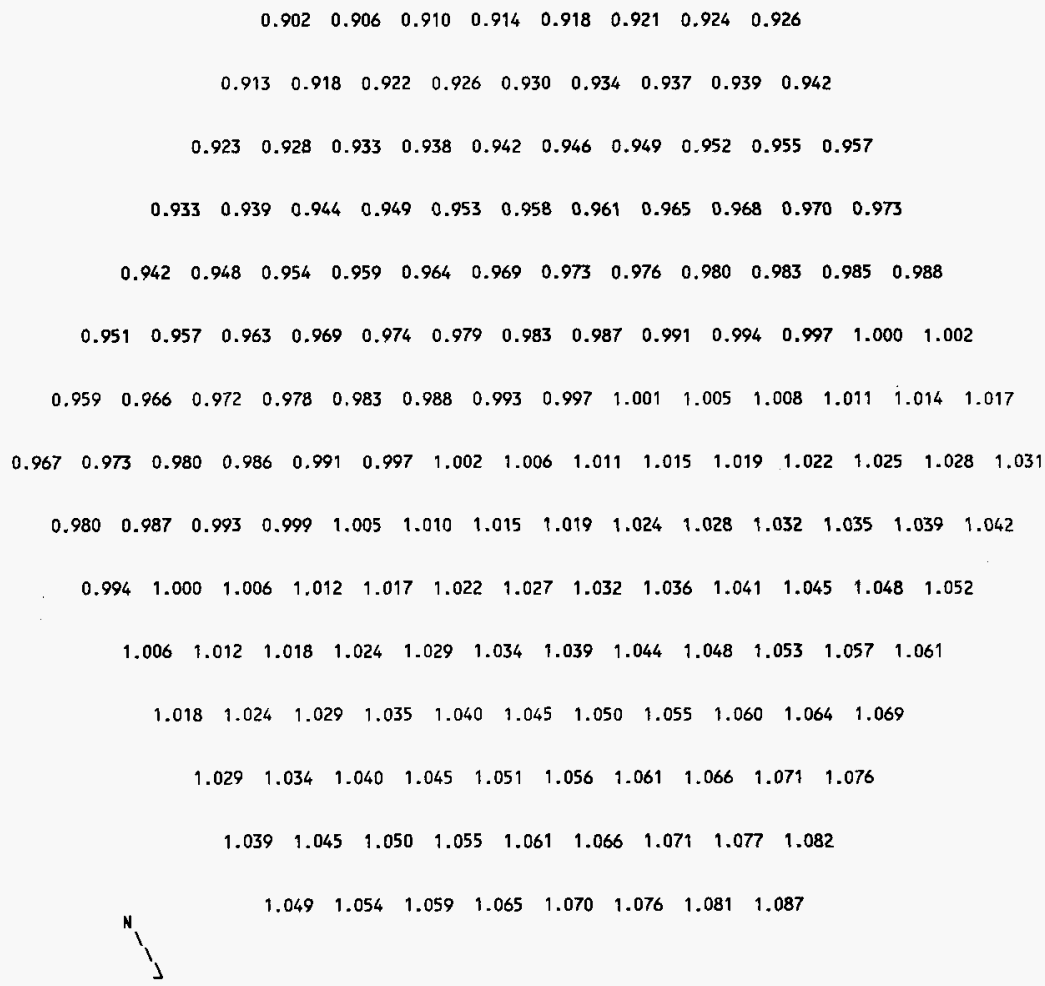




\section{WHC-SD-FF-ANAL-009 REV 0}

Table ElOA-3,6. Fission Power Distribution by Pin in MFA-2 at EOC 1OA-3

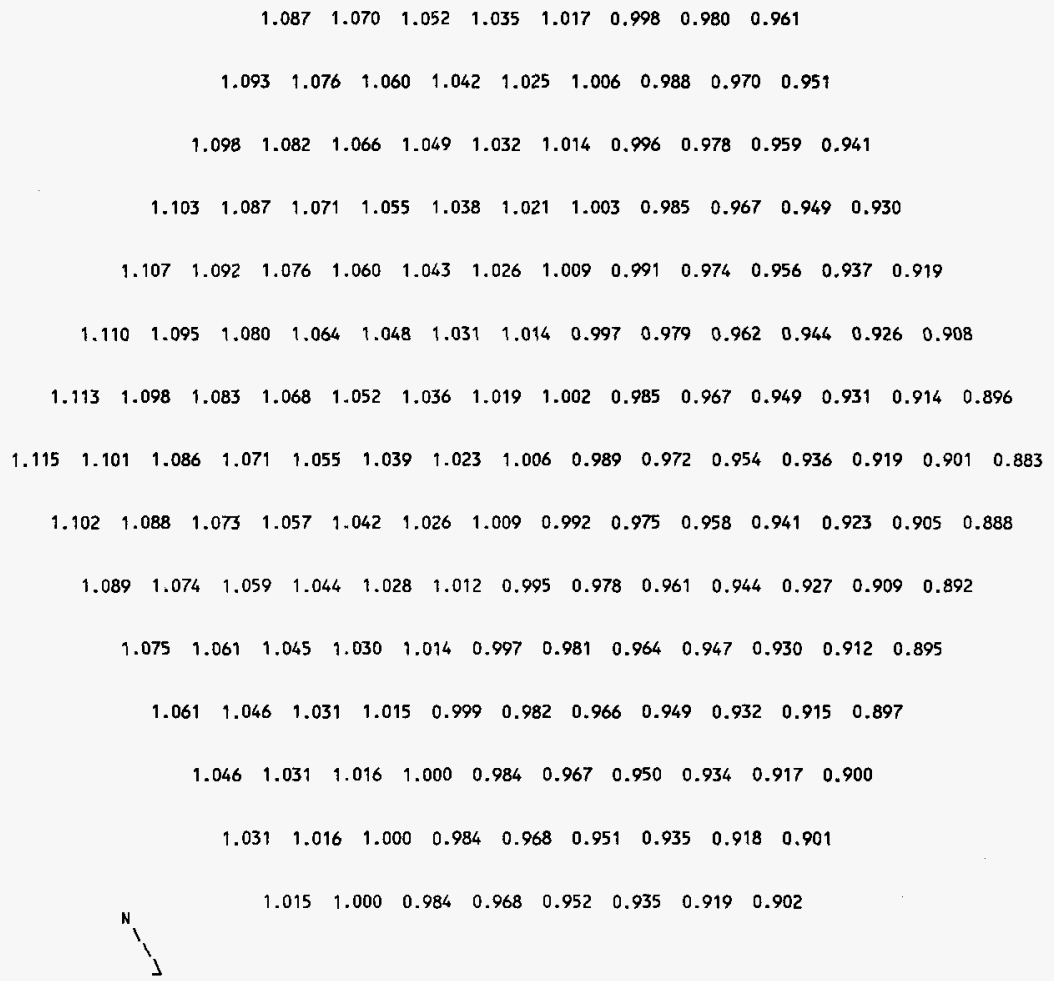


WHC-SD-FF-ANAL-009 REV 0

Table E10A-3.7. Total Flux Distribution by Pin in MFA-1 at EOC 10A-3

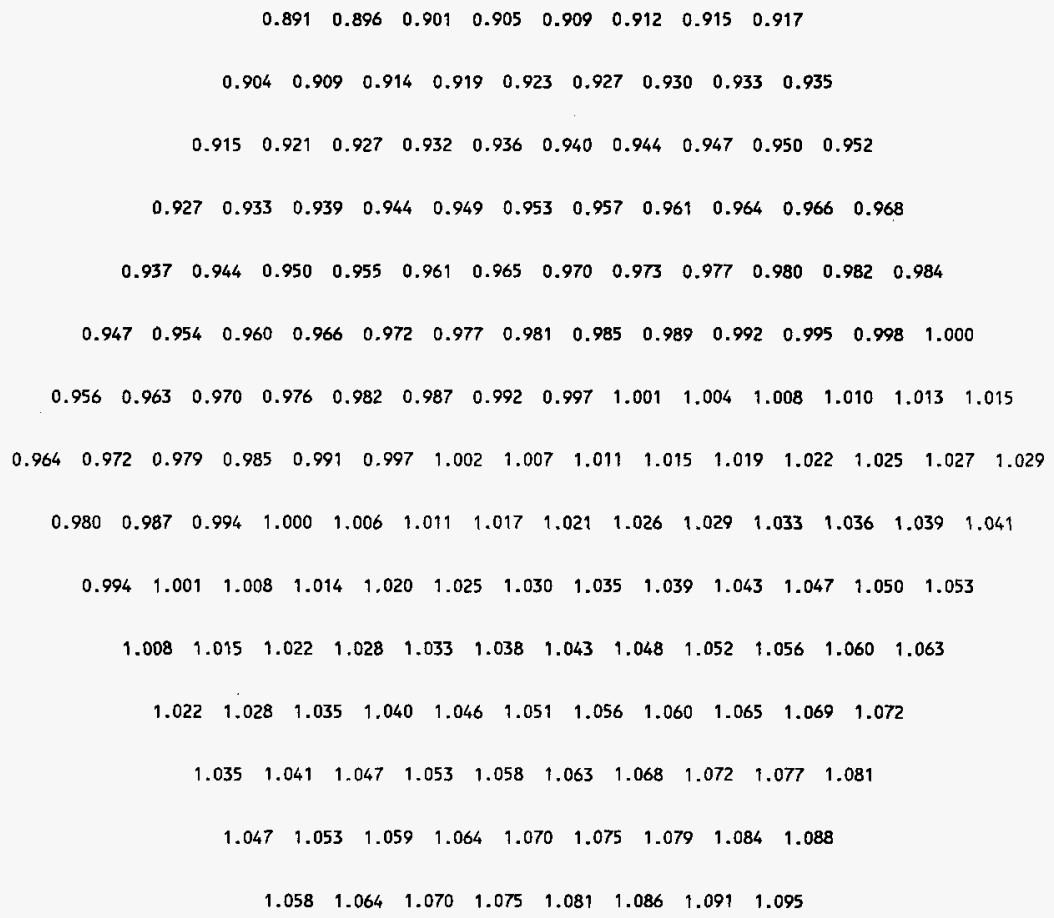


Table E10A-3.8. Total Flux Distribution by Pin in MFA-2 at EOC 1OA-3

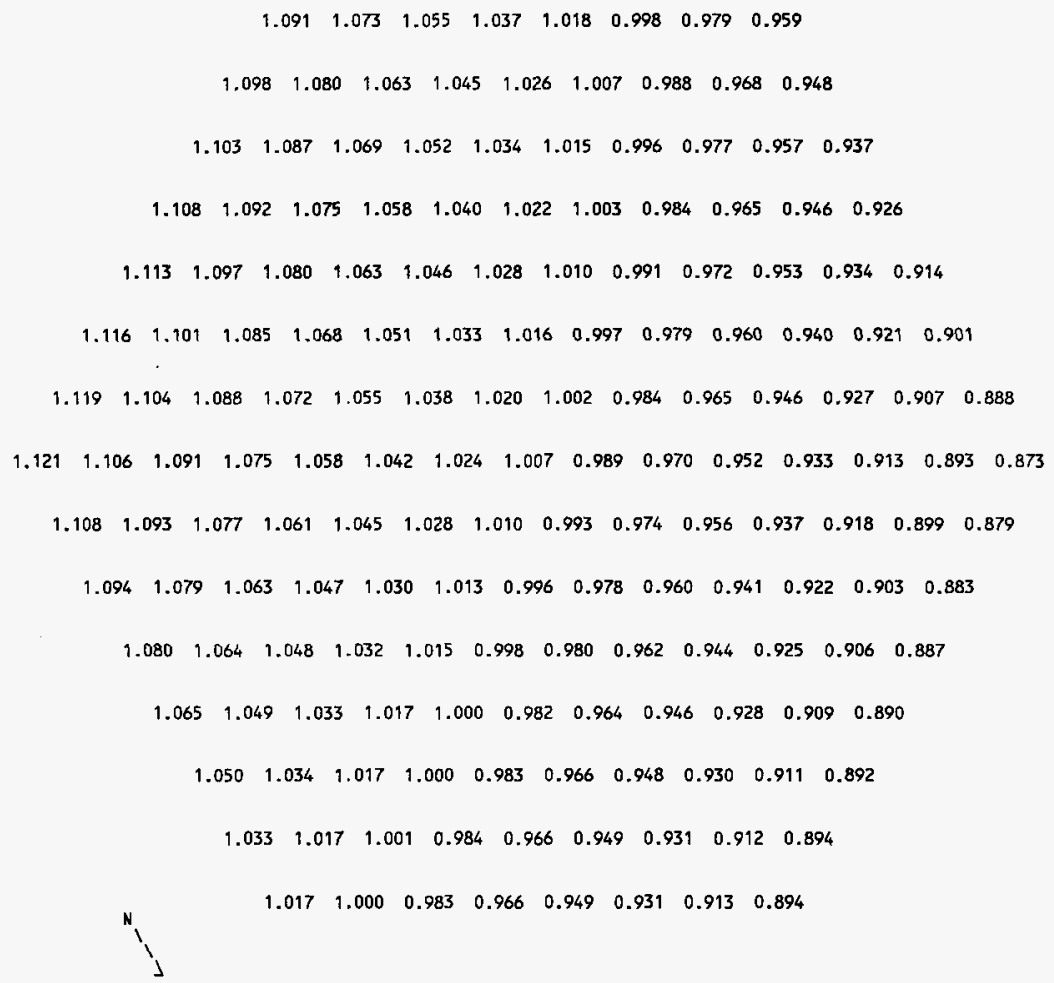


Table E10A-3.9. MFA-1 and MFA-2 Duct Wall Flux Greater Than $0.1 \mathrm{MeV}$ at EOC 10A-3

$\begin{array}{ccc}\text { SIDE } & \begin{array}{c}\text { FLUX }>0.1 \\ \text { MFA-1 }\end{array} & \begin{array}{c}\text { MEV } \\ \left(\mathrm{n} / \mathrm{cm}^{2}-\mathrm{sec}\right) \\ \text { MFA-2 }\end{array} \\ \text { E } & 1.8821 \mathrm{E}+15 & 2.1351 \mathrm{E}+15 \\ \text { SE } & 1.6818 \mathrm{1}+15 & 2.2215 \mathrm{E}+15 \\ \text { SW } & 1.6127 \mathrm{E}+15 & 2.0142 \mathrm{E}+15 \\ \text { W } & 1.7735 \mathrm{E}+15 & 1.7575 \mathrm{E}+15 \\ \text { NW } & 1.9921 \mathrm{E}+15 & 1.6805 \mathrm{E}+15 \\ \text { NE } & 2.0485 \mathrm{E}+15 & 1.8385 \mathrm{E}+15\end{array}$


Table E10A-3.17. Composition of MFA-1 at EOC 10A-3

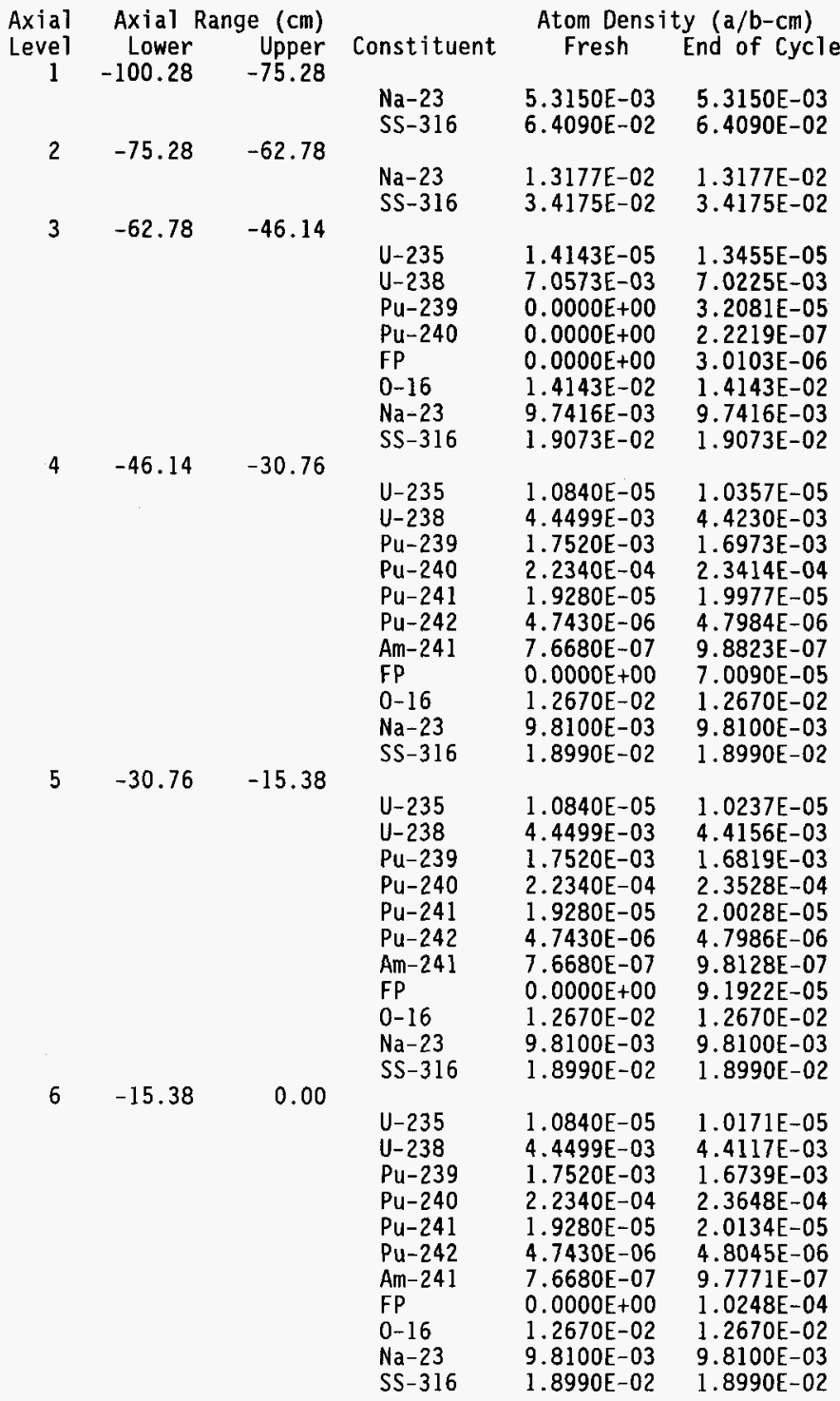


$0.00 \quad 15.38$

8

$15.38 \quad 30.76$

$\begin{array}{ll}\text { U-235 } & 1.0840 \mathrm{E}-05 \\ \mathrm{U}-238 & 4.4499 \mathrm{E}-03 \\ \mathrm{Pu}-239 & 1.7520 \mathrm{E}-03 \\ \mathrm{Pu}-240 & 2.2340 \mathrm{E}-04 \\ \mathrm{Pu}-241 & 1.9280 \mathrm{E}-05 \\ \mathrm{Pu}-242 & 4.7430 \mathrm{E}-06 \\ \mathrm{Am}-241 & 7.6580 \mathrm{E}-07 \\ \mathrm{FP} & 0.0000 \mathrm{E}+00 \\ \mathrm{O}-16 & 1.2670 \mathrm{E}-02 \\ \mathrm{Na}-23 & 9.8100 \mathrm{E}-03 \\ \text { SS-316 } & 1.8990 \mathrm{E}-02\end{array}$

$1.0192 \mathrm{E}-05$

$4.4130 \mathrm{E}-03$

$1.6764 \mathrm{E}-03$

2.3603E-04

2.0094E-05

$4.8021 \mathrm{E}-06$

9.7885E-07

9. $9159 \mathrm{E}-05$

$1.2670 \mathrm{E}-02$

$9.8100 \mathrm{E}-03$

$1.8990 \mathrm{E}-02$

U-235 $\quad 1.0840 \mathrm{E}-05 \quad 1.0297 \mathrm{E}-05$

$\mathrm{U}-238 \quad 4.4499 \mathrm{E}-03 \quad 4.4190 \mathrm{E}-03$

$\begin{array}{lll}\mathrm{Pu}-239 & 1.7520 \mathrm{E}-03 & 1.6888 \mathrm{E}-03\end{array}$

Pu-240 2.2340E-04 2.3404E-04

$\mathrm{Pu}-241 \quad 1.9280 \mathrm{E}-05 \quad 1.9917 \mathrm{E}-05$

Pu-242 4.7430E-06 4.7919E-06

$\begin{array}{lll}\text { Am-241 } & 7.6680 \mathrm{E}-07 & 9.8439 \mathrm{E}-07\end{array}$

$\begin{array}{lll}\mathrm{FP} & 0.0000 \mathrm{E}+00 & 8.2964 \mathrm{E}-05\end{array}$

$0-16 \quad 1.2670 \mathrm{E}-02 \quad 1.2670 \mathrm{E}-02$

$\mathrm{Na}-23 \quad 9.8100 \mathrm{E}-03 \quad 9.8100 \mathrm{E}-03$

SS-316 $1.8990 \mathrm{E}-02 \quad 1.8990 \mathrm{E}-02$

$\begin{array}{lll}9 & 30.76 & 46.14\end{array}$

U-235 $\quad 1.0840 \mathrm{E}-05 \quad 1.0441 \mathrm{E}-05$

U-238 4.4499E-03 4.4277E-03

$\begin{array}{lll}\mathrm{Pu}-239 & 1.7520 \mathrm{E}-03 & 1.7067 \mathrm{E}-03\end{array}$

Pu-240 2.2340E-04 2.3204E-04

Pu-241 $1.9280 E-05 \quad 1.9774 E-05$

Pu-242 4.7430E-06 4.7859E-06

Am-241 7.6680E-07 9.9251E-07

FP $\quad 0.0000 \mathrm{E}+00 \quad 5.8275 \mathrm{E}-05$

$\begin{array}{lll}0-16 & 1.2670 \mathrm{E}-02 & 1.2670 \mathrm{E}-02\end{array}$

Na-23 $\quad 9.8100 \mathrm{E}-03 \quad 9.8100 \mathrm{E}-03$

$\begin{array}{lll}10 & 46.14 & 62.78\end{array}$

SS-316 $1.8990 \mathrm{E}-02 \quad 1.8990 \mathrm{E}-02$

$\begin{array}{lll}\text { U-235 } & 1.4143 \mathrm{E}-05 & 1.3681 \mathrm{E}-05 \\ \mathrm{U}-238 & 7.0573 \mathrm{E}-03 & 7.0332 \mathrm{E}-03 \\ \mathrm{Pu}-239 & 0.0000 \mathrm{E}+00 & 2.2166 \mathrm{E}-05 \\ \mathrm{Pu}-240 & 0.0000 \mathrm{E}+00 & 9.8597 \mathrm{E}-08 \\ \mathrm{FP} & 0.0000 \mathrm{E}+00 & 2.1588 \mathrm{E}-06 \\ \mathrm{O}-16 & 1.4143 \mathrm{E}-02 & 1.4143 \mathrm{E}-02 \\ \mathrm{Na}-23 & 9.7416 \mathrm{E}-03 & 9.7416 \mathrm{E}-03 \\ \mathrm{SS}-316 & 1.9073 \mathrm{E}-02 & 1.9073 \mathrm{E}-02\end{array}$

$\begin{array}{lll}11 & 62.78 & 116.00\end{array}$

$\begin{array}{lll}\mathrm{Na}-23 & 8.2400 \mathrm{E}-03 & 8.2400 \mathrm{E}-03 \\ \mathrm{SS}-316 & 2.5562 \mathrm{E}-02 & 2.5562 \mathrm{E}-02\end{array}$


Table EIOA-3.18. Composition of MFA-2 at EOC 10A-3

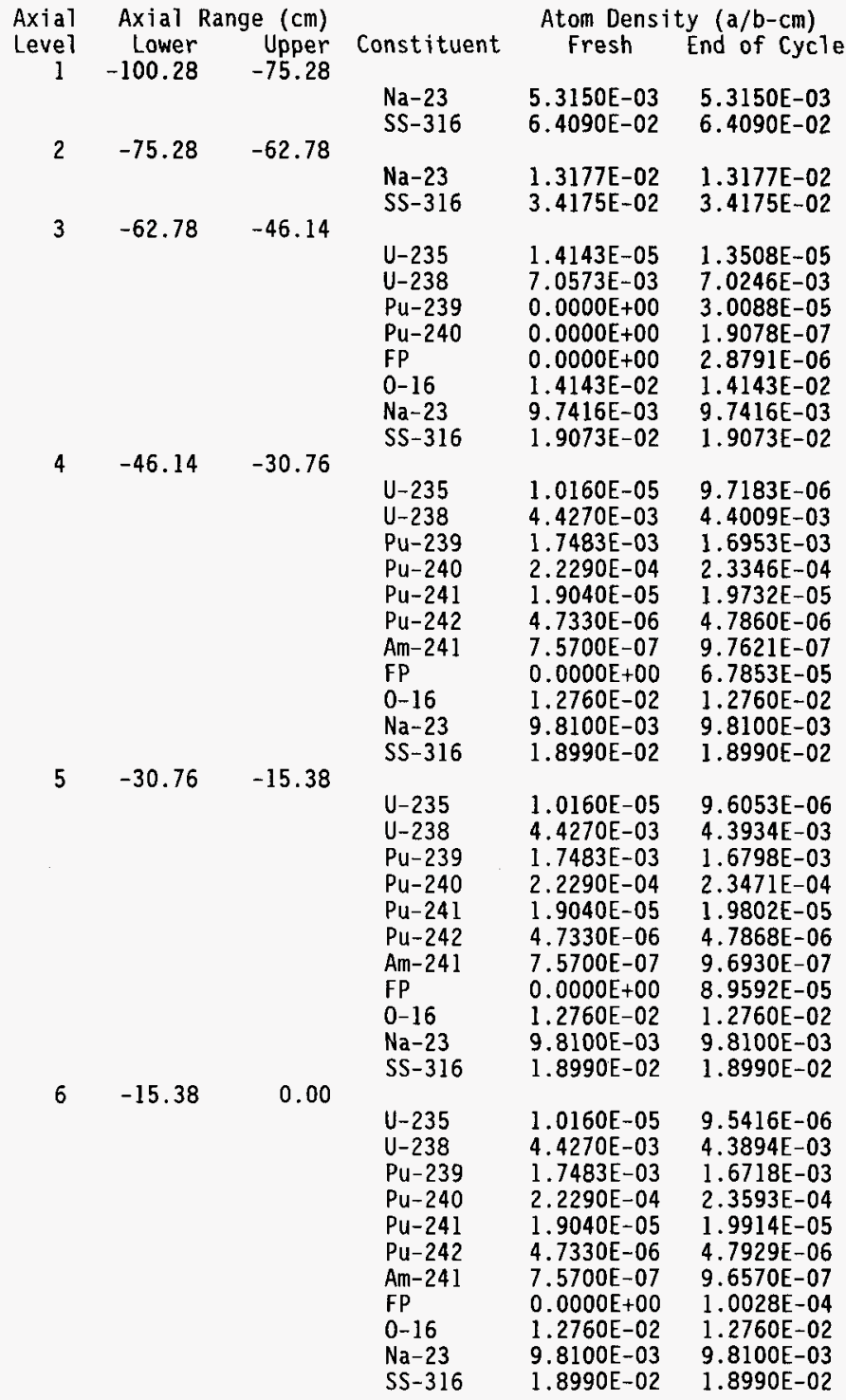




\begin{tabular}{|c|c|c|c|c|c|}
\hline \multirow[t]{2}{*}{7} & 0.00 & 15.38 & & & \\
\hline & & & $\begin{array}{l}\mathrm{U}-235 \\
\mathrm{U}-238 \\
\mathrm{Pu}-239 \\
\mathrm{Pu}-240 \\
\mathrm{Pu}-241 \\
\mathrm{Pu}-242 \\
\mathrm{Am}-241 \\
\mathrm{FP} \\
0-16 \\
\mathrm{Na}-23 \\
\mathrm{SS}-316\end{array}$ & $\begin{array}{l}1.0160 \mathrm{E}-05 \\
4.4270 \mathrm{E}-03 \\
1.7483 \mathrm{E}-03 \\
2.2290 \mathrm{E}-04 \\
1.9040 \mathrm{E}-05 \\
4.7330 \mathrm{E}-06 \\
7.5700 \mathrm{E}-07 \\
0.0000 \mathrm{E}+00 \\
1.2760 \mathrm{E}-02 \\
9.8100 \mathrm{E}-03 \\
1.8990 \mathrm{E}-02\end{array}$ & $\begin{array}{l}9.5603 \mathrm{E}-06 \\
4.3906 \mathrm{E}-03 \\
1.6740 \mathrm{E}-03 \\
2.3553 \mathrm{E}-04 \\
1.9876 \mathrm{E}-05 \\
4.7907 \mathrm{E}-06 \\
9.6671 \mathrm{E}-07 \\
9.7279 \mathrm{E}-05 \\
1.2760 \mathrm{E}-02 \\
9.8100 \mathrm{E}-03 \\
1.8990 \mathrm{E}-02\end{array}$ \\
\hline \multirow[t]{2}{*}{8} & 15.38 & 30.76 & & & \\
\hline & & & $\begin{array}{l}U-235 \\
U-238 \\
P u-239 \\
P u-240 \\
P u-241 \\
P u-242 \\
\mathrm{Am}-241 \\
\text { FP } \\
0-16 \\
\mathrm{Na}-23 \\
\mathrm{SS}-316\end{array}$ & $\begin{array}{l}1.0160 \mathrm{E}-05 \\
4.4270 \mathrm{E}-03 \\
1.7483 \mathrm{E}-03 \\
2.2290 \mathrm{E}-04 \\
1.9040 \mathrm{E}-05 \\
4.7330 \mathrm{E}-06 \\
7.5700 \mathrm{E}-07 \\
0.0000 \mathrm{E}+00 \\
1.2760 \mathrm{E}-02 \\
9.8100 \mathrm{E}-03 \\
1.8990 \mathrm{E}-02\end{array}$ & $\begin{array}{l}9.6540 \mathrm{E}-06 \\
4.3964 \mathrm{E}-03 \\
1.6858 \mathrm{E}-03 \\
2.3363 \mathrm{E}-04 \\
1.9705 \mathrm{E}-05 \\
4.7813 \mathrm{E}-06 \\
9.7198 \mathrm{E}-07 \\
8.1783 \mathrm{E}-05 \\
1.2760 \mathrm{E}-02 \\
9.8100 \mathrm{E}-03 \\
1.8990 \mathrm{E}-02\end{array}$ \\
\hline \multirow[t]{2}{*}{9} & 30.76 & 46.14 & & & \\
\hline & & & $\begin{array}{l}\mathrm{U}-235 \\
\mathrm{U}-238 \\
\mathrm{Pu}-239 \\
\mathrm{Pu}-240 \\
\mathrm{Pu}-241 \\
\mathrm{Pu}-242 \\
\mathrm{Am}-241 \\
\mathrm{FP} \\
0-16 \\
\mathrm{Na}-23 \\
\mathrm{SS}-316\end{array}$ & $\begin{array}{l}1.0160 \mathrm{E}-05 \\
4.4270 \mathrm{E}-03 \\
1.7483 \mathrm{E}-03 \\
2.2290 \mathrm{E}-04 \\
1.9040 \mathrm{E}-05 \\
4.7330 \mathrm{E}-06 \\
7.5700 \mathrm{E}-07 \\
0.0000 \mathrm{E}+00 \\
1.2760 \mathrm{E}-02 \\
9.8100 \mathrm{E}-03 \\
1.8990 \mathrm{E}-02\end{array}$ & $\begin{array}{l}9.7874 \mathrm{E}-06 \\
4.4049 \mathrm{E}-03 \\
1.7035 \mathrm{E}-03 \\
2.3166 \mathrm{E}-04 \\
1.9557 \mathrm{E}-05 \\
4.7755 \mathrm{E}-06 \\
9.7988 \mathrm{E}-07 \\
5.7644 \mathrm{E}-05 \\
1.2760 \mathrm{E}-02 \\
9.8100 \mathrm{E}-03 \\
1.8990 \mathrm{E}-02\end{array}$ \\
\hline \multirow[t]{2}{*}{10} & 46.14 & 62.78 & & & \\
\hline & & & $\begin{array}{l}U-235 \\
U-238 \\
P u-239 \\
P u-240 \\
F P \\
0-16 \\
N a-23 \\
S S-316\end{array}$ & $\begin{array}{l}1.4143 \mathrm{E}-05 \\
7.0573 \mathrm{E}-03 \\
0.0000 \mathrm{E}+00 \\
0.0000 \mathrm{E}+00 \\
0.0000 \mathrm{E}+00 \\
1.4143 \mathrm{E}-02 \\
9.7416 \mathrm{E}-03 \\
1.9073 \mathrm{E}-02\end{array}$ & $\begin{array}{l}1.3691 \mathrm{E}-05 \\
7.0334 \mathrm{E}-03 \\
2.1955 \mathrm{E}-05 \\
9.5959 \mathrm{E}-08 \\
2.1603 \mathrm{E}-06 \\
1.4143 \mathrm{E}-02 \\
9.7416 \mathrm{E}-03 \\
1.9073 \mathrm{E}-02\end{array}$ \\
\hline 11 & & & $\begin{array}{l}\mathrm{Na}-23 \\
\mathrm{SS}-316\end{array}$ & $\begin{array}{l}8.2400 \mathrm{E}-03 \\
2.5562 \mathrm{E}-02\end{array}$ & $\begin{array}{l}8.2400 E-03 \\
2.5562 E-02\end{array}$ \\
\hline
\end{tabular}


Table B10A-4.1. Fission Power Generated in MFA-1, MFA-2 and Neighboring Assemblies at $\mathrm{BOC} 10 \mathrm{~A}-4$

\begin{tabular}{lrrrrr} 
CORE & $* * * * * *$ & \multicolumn{2}{c}{ POWER IN MEGAWATTS } & $* * * * * *$ \\
POS. & BELOW CORE & \multicolumn{2}{c}{ IN CORE } & ABOVE CORE & \multicolumn{1}{c}{ TOTAL PWR } \\
1506 & $2.959 \mathrm{E}-02$ & $3.863 \mathrm{E}+00$ & $2.031 \mathrm{E}-02$ & $3.913 \mathrm{E}+00$ \\
1404 & $8.374 \mathrm{E}-02$ & $4.628 \mathrm{E}+00$ & $4.589 \mathrm{E}-02$ & $4.757 \mathrm{E}+00$ \\
1405 & $9.610 \mathrm{E}-03$ & $3.864 \mathrm{E}+00$ & $7.175 \mathrm{E}-03$ & $3.881 \mathrm{E}+00$ \\
1505 & $1.135 \mathrm{E}-02$ & $3.538 \mathrm{E}+00$ & $6.690 \mathrm{E}-03$ & $3.556 \mathrm{E}+00$ \\
1507 & $9.833 \mathrm{E}-03$ & $3.270 \mathrm{E}+00$ & $7.354 \mathrm{E}-03$ & $3.288 \mathrm{E}+00$ \\
1606 & $6.255 \mathrm{E}-03$ & $3.440 \mathrm{E}+00$ & $4.567 \mathrm{E}-03$ & $3.451 \mathrm{E}+00$ \\
1607 & $9.175 \mathrm{E}-03$ & $2.840 \mathrm{E}+00$ & $6.636 \mathrm{E}-03$ & $2.855 \mathrm{E}+00$ \\
2507 & $3.109 \mathrm{E}-02$ & $4.207 \mathrm{E}+00$ & $2.281 \mathrm{E}-02$ & $4.261 \mathrm{E}+00$ \\
2405 & $7.328 \mathrm{E}-02$ & $4.449 \mathrm{E}+00$ & $4.264 \mathrm{E}-02$ & $4.565 \mathrm{E}+00$ \\
2506 & $1.160 \mathrm{E}-02$ & $3.878 \mathrm{E}+00$ & $8.209 \mathrm{E}-03$ & $3.897 \mathrm{E}+00$ \\
2508 & $1.141 \mathrm{E}-02$ & $4.612 \mathrm{E}+00$ & $8.388 \mathrm{E}-03$ & $4.631 \mathrm{E}+00$ \\
2607 & $8.010 \mathrm{E}-03$ & $3.585 \mathrm{E}+00$ & $5.996 \mathrm{E}-03$ & $3.599 \mathrm{E}+00$ \\
2608 & $5.213 \mathrm{E}-03$ & $3.489 \mathrm{E}+00$ & $4.338 \mathrm{E}-03$ & $3.499 \mathrm{E}+00$ \\
2609 & $1.095 \mathrm{E}-02$ & $3.634 \mathrm{E}+00$ & $7.128 \mathrm{E}-03$ & $3.652 \mathrm{E}+00$
\end{tabular}

Table B1OA-4.2. Assembly Averaged Total Flux for MFA-1 and MFA-2 at BOC 10A-4

$\begin{array}{ccc} & \text { Core } & \text { Total Flux } \\ \text { Assembly } & \text { Pos. } & \left(\mathrm{n} / \mathrm{cm}^{2}-\mathrm{sec}\right) \\ \text { MFA-1 } & 1506 & 2.861 \mathrm{E}+15 \\ \text { MFA-2 } & 2507 & 3.107 \mathrm{E}+15\end{array}$


Table BlOA-4.3. Axial Distribution of Total Flux, Fast Flux and Power in MFA-1 at BOC 10A-4

$\begin{array}{rccc}\text { z }(\mathrm{cm}) & \text { Total Flux } & \text { Flux }>0.1 \text { MeV } & \text { Power } \\ -97.78 & 2.7595 \mathrm{E}-02 & 1.3987 \mathrm{E}-02 & 0.0000 \mathrm{E}+00 \\ -92.78 & 5.9310 \mathrm{E}-02 & 2.7007 \mathrm{E}-02 & 0.0000 \mathrm{E}+00 \\ -87.78 & 9.5095 \mathrm{E}-02 & 4.3215 \mathrm{E}-02 & 0.0000 \mathrm{E}+00 \\ -82.78 & 1.3765 \mathrm{E}-01 & 6.4455 \mathrm{E}-02 & 0.0000 \mathrm{E}+00 \\ -77.78 & 1.8968 \mathrm{E}-01 & 9.3017 \mathrm{E}-02 & 0.0000 \mathrm{E}+00 \\ -72.15 & 2.4851 \mathrm{E}-01 & 1.2993 \mathrm{E}-01 & 0.0000 \mathrm{E}+00 \\ -65.90 & 3.0691 \mathrm{E}-01 & 1.7628 \mathrm{E}-01 & 0.0000 \mathrm{E}+00 \\ -60.35 & 3.6841 \mathrm{E}-01 & 2.3856 \mathrm{E}-01 & 2.8315 \mathrm{E}-02 \\ -55.49 & 4.5020 \mathrm{E}-01 & 3.2302 \mathrm{E}-01 & 3.7039 \mathrm{E}-02 \\ -50.62 & 5.4584 \mathrm{E}-01 & 4.3389 \mathrm{E}-01 & 5.1220 \mathrm{E}-02 \\ -47.17 & 6.2322 \mathrm{E}-01 & 5.3942 \mathrm{E}-01 & 6.8216 \mathrm{E}-02 \\ -43.58 & 7.0484 \mathrm{E}-01 & 6.5960 \mathrm{E}-01 & 7.4599 \mathrm{E}-01 \\ -38.45 & 8.2181 \mathrm{E}-01 & 8.0374 \mathrm{E}-01 & 8.3666 \mathrm{E}-01 \\ -33.32 & 9.2983 \mathrm{E}-01 & 9.2595 \mathrm{E}-01 & 9.3643 \mathrm{E}-01 \\ -28.20 & 1.0239 \mathrm{E}+00 & 1.0272 \mathrm{E}+00 & 1.0192 \mathrm{E}+00 \\ -23.07 & 1.1021 \mathrm{E}+00 & 1.1093 \mathrm{E}+00 & 1.0953 \mathrm{E}+00 \\ -17.94 & 1.1629 \mathrm{E}+00 & 1.1720 \mathrm{E}+00 & 1.1548 \mathrm{E}+00 \\ -12.82 & 1.2054 \mathrm{E}+00 & 1.2151 \mathrm{E}+00 & 1.1917 \mathrm{E}+00 \\ -7.69 & 1.2295 \mathrm{E}+00 & 1.2396 \mathrm{E}+00 & 1.2152 \mathrm{E}+00 \\ -2.56 & 1.2350 \mathrm{E}+00 & 1.2452 \mathrm{E}+00 & 1.2204 \mathrm{E}+00 \\ 2.56 & 1.2218 \mathrm{E}+00 & 1.2323 \mathrm{E}+00 & 1.2088 \mathrm{E}+00 \\ 7.69 & 1.1903 \mathrm{E}+00 & 1.2009 \mathrm{E}+00 & 1.1776 \mathrm{E}+00 \\ 12.82 & 1.1412 \mathrm{E}+00 & 1.1519 \mathrm{E}+00 & 1.1290 \mathrm{E}+00 \\ 17.94 & 1.0759 \mathrm{E}+00 & 1.0865 \mathrm{E}+00 & 1.0712 \mathrm{E}+00 \\ 23.07 & 9.9562 \mathrm{E}-01 & 1.0049 \mathrm{E}+00 & 9.9181 \mathrm{E}-01 \\ 28.20 & 9.0249 \mathrm{E}-01 & 9.0877 \mathrm{E}-01 & 9.0007 \mathrm{E}-01 \\ 33.32 & 7.9887 \mathrm{E}-01 & 7.9953 \mathrm{E}-01 & 8.0638 \mathrm{E}-01 \\ 38.45 & 6.8706 \mathrm{E}-01 & 6.7678 \mathrm{E}-01 & 6.9947 \mathrm{E}-01 \\ 43.58 & 5.7143 \mathrm{E}-01 & 5.4065 \mathrm{E}-01 & 6.0009 \mathrm{E}-01 \\ 47.17 & 4.9264 \mathrm{E}-01 & 4.3380 \mathrm{E}-01 & 5.1742 \mathrm{E}-02 \\ 50.62 & 4.1825 \mathrm{E}-01 & 3.4020 \mathrm{E}-01 & 3.6926 \mathrm{E}-02 \\ 55.49 & 3.2674 \mathrm{E}-01 & 2.4234 \mathrm{E}-01 & 2.4441 \mathrm{E}-02 \\ 60.35 & 2.4774 \mathrm{E}-01 & 1.6782 \mathrm{E}-01 & 1.6592 \mathrm{E}-02 \\ 64.98 & 1.9943 \mathrm{E}-01 & 1.2549 \mathrm{E}-01 & 0.0000 \mathrm{E}+00 \\ 69.39 & 1.7128 \mathrm{E}-01 & 1.0332 \mathrm{E}-01 & 0.0000 \mathrm{E}+00 \\ 76.46 & 1.3099 \mathrm{E}-01 & 7.3607 \mathrm{E}-02 & 0.0000 \mathrm{E}+00 \\ 86.80 & 8.5984 \mathrm{E}-02 & 4.4584 \mathrm{E}-02 & 0.0000 \mathrm{E}+00 \\ 98.14 & 4.9848 \mathrm{E}-02 & 2.4457 \mathrm{E}-02 & 0.0000 \mathrm{E}+00 \\ 110.00 & 2.1429 \mathrm{E}-02 & 1.0999 \mathrm{E}-02 & 0.0000 \mathrm{E}+00\end{array}$


Table B10A-4.4. Axial Distribution of Total Flux, Fast Flux and Power in MFA-2 at BOC 10A-4

$\begin{array}{rccc}z(\mathrm{~cm}) & \text { Total Flux } & \text { Flux }>0.1 \text { MeV } & \text { Power } \\ -97.78 & 2.5060 \mathrm{E}-02 & 1.2741 \mathrm{E}-02 & 0.0000 \mathrm{E}+00 \\ -92.78 & 5.3832 \mathrm{E}-02 & 2.4595 \mathrm{E}-02 & 0.0000 \mathrm{E}+00 \\ -87.78 & 8.6354 \mathrm{E}-02 & 3.9368 \mathrm{E}-02 & 0.0000 \mathrm{E}+00 \\ -82.78 & 1.2523 \mathrm{E}-01 & 5.8792 \mathrm{E}-02 & 0.0000 \mathrm{E}+00 \\ -77.78 & 1.7333 \mathrm{E}-01 & 8.5065 \mathrm{E}-02 & 0.0000 \mathrm{E}+00 \\ -72.15 & 2.2887 \mathrm{E}-01 & 1.1941 \mathrm{E}-01 & 0.0000 \mathrm{E}+00 \\ -65.90 & 2.8629 \mathrm{E}-01 & 1.6365 \mathrm{E}-01 & 0.0000 \mathrm{E}+00 \\ -60.35 & 3.4853 \mathrm{E}-01 & 2.2408 \mathrm{E}-01 & 2.6713 \mathrm{E}-02 \\ -55.49 & 4.3044 \mathrm{E}-01 & 3.0680 \mathrm{E}-01 & 3.5515 \mathrm{E}-02 \\ -50.62 & 5.2667 \mathrm{E}-01 & 4.1681 \mathrm{E}-01 & 4.9790 \mathrm{E}-02 \\ -47.17 & 6.0506 \mathrm{E}-01 & 5.2251 \mathrm{E}-01 & 6.6856 \mathrm{E}-02 \\ -43.58 & 6.8785 \mathrm{E}-01 & 6.4307 \mathrm{E}-01 & 7.3100 \mathrm{E}-01 \\ -38.45 & 8.0698 \mathrm{E}-01 & 7.8882 \mathrm{E}-01 & 8.2217 \mathrm{E}-01 \\ -33.32 & 9.1736 \mathrm{E}-01 & 9.1336 \mathrm{E}-01 & 9.2367 \mathrm{E}-01 \\ -28.20 & 1.0139 \mathrm{E}+00 & 1.0174 \mathrm{E}+00 & 1.0086 \mathrm{E}+00 \\ -23.07 & 1.0948 \mathrm{E}+00 & 1.1024 \mathrm{E}+00 & 1.0873 \mathrm{E}+00 \\ -17.94 & 1.1585 \mathrm{E}+00 & 1.1681 \mathrm{E}+00 & 1.1497 \mathrm{E}+00 \\ -12.82 & 1.2038 \mathrm{E}+00 & 1.2140 \mathrm{E}+00 & 1.1895 \mathrm{E}+00 \\ -7.69 & 1.2303 \mathrm{E}+00 & 1.2408 \mathrm{E}+00 & 1.2154 \mathrm{E}+00 \\ -2.56 & 1.2375 \mathrm{E}+00 & 1.2483 \mathrm{E}+00 & 1.2225 \mathrm{E}+00 \\ 2.56 & 1.2258 \mathrm{E}+00 & 1.2369 \mathrm{E}+00 & 1.2121 \mathrm{E}+00 \\ 7.69 & 1.1956 \mathrm{E}+00 & 1.2068 \mathrm{E}+00 & 1.1822 \mathrm{E}+00 \\ 12.82 & 1.1479 \mathrm{E}+00 & 1.1589 \mathrm{E}+00 & 1.1353 \mathrm{E}+00 \\ 17.94 & 1.0840 \mathrm{E}+00 & 1.0946 \mathrm{E}+00 & 1.0788 \mathrm{E}+00 \\ 23.07 & 1.0047 \mathrm{E}+00 & 1.0137 \mathrm{E}+00 & 1.0006 \mathrm{E}+00 \\ 28.20 & 9.1185 \mathrm{E}-01 & 9.1753 \mathrm{E}-01 & 9.0926 \mathrm{E}-01 \\ 33.32 & 8.0764 \mathrm{E}-01 & 8.0748 \mathrm{E}-01 & 8.1520 \mathrm{E}-01 \\ 38.45 & 6.9441 \mathrm{E}-01 & 6.8304 \mathrm{E}-01 & 7.0766 \mathrm{E}-01 \\ 43.58 & 5.7696 \mathrm{E}-01 & 5.4466 \mathrm{E}-01 & 6.0898 \mathrm{E}-01 \\ 47.17 & 4.9702 \mathrm{E}-01 & 4.3591 \mathrm{E}-01 & 5.3237 \mathrm{E}-02 \\ 50.62 & 4.2160 \mathrm{E}-01 & 3.4074 \mathrm{E}-01 & 3.8040 \mathrm{E}-02 \\ 55.49 & 3.2928 \mathrm{E}-01 & 2.4201 \mathrm{E}-01 & 2.5221 \mathrm{E}-02 \\ 60.35 & 2.4974 \mathrm{E}-01 & 1.6744 \mathrm{E}-01 & 1.7153 \mathrm{E}-02 \\ 64.98 & 2.0073 \mathrm{E}-01 & 1.2495 \mathrm{E}-01 & 0.0000 \mathrm{E}+00 \\ 69.39 & 1.7166 \mathrm{E}-01 & 1.0240 \mathrm{E}-01 & 0.0000 \mathrm{E}+00 \\ 76.46 & 1.3019 \mathrm{E}-01 & 7.2258 \mathrm{E}-02 & 0.0000 \mathrm{E}+00 \\ 86.80 & 8.4695 \mathrm{E}-02 & 4.3239 \mathrm{E}-02 & 0.0000 \mathrm{E}+00 \\ 98.14 & 4.8685 \mathrm{E}-02 & 2.3423 \mathrm{E}-02 & 0.0000 \mathrm{E}+00 \\ 110.00 & 2.0618 \mathrm{E}-02 & 1.0349 \mathrm{E}-02 & 0.0000 \mathrm{E}+00 \\ & & & \end{array}$


Table B10A-4.5. Fission Power Distribution by Pin in MFA-1 at BOC 10A-4

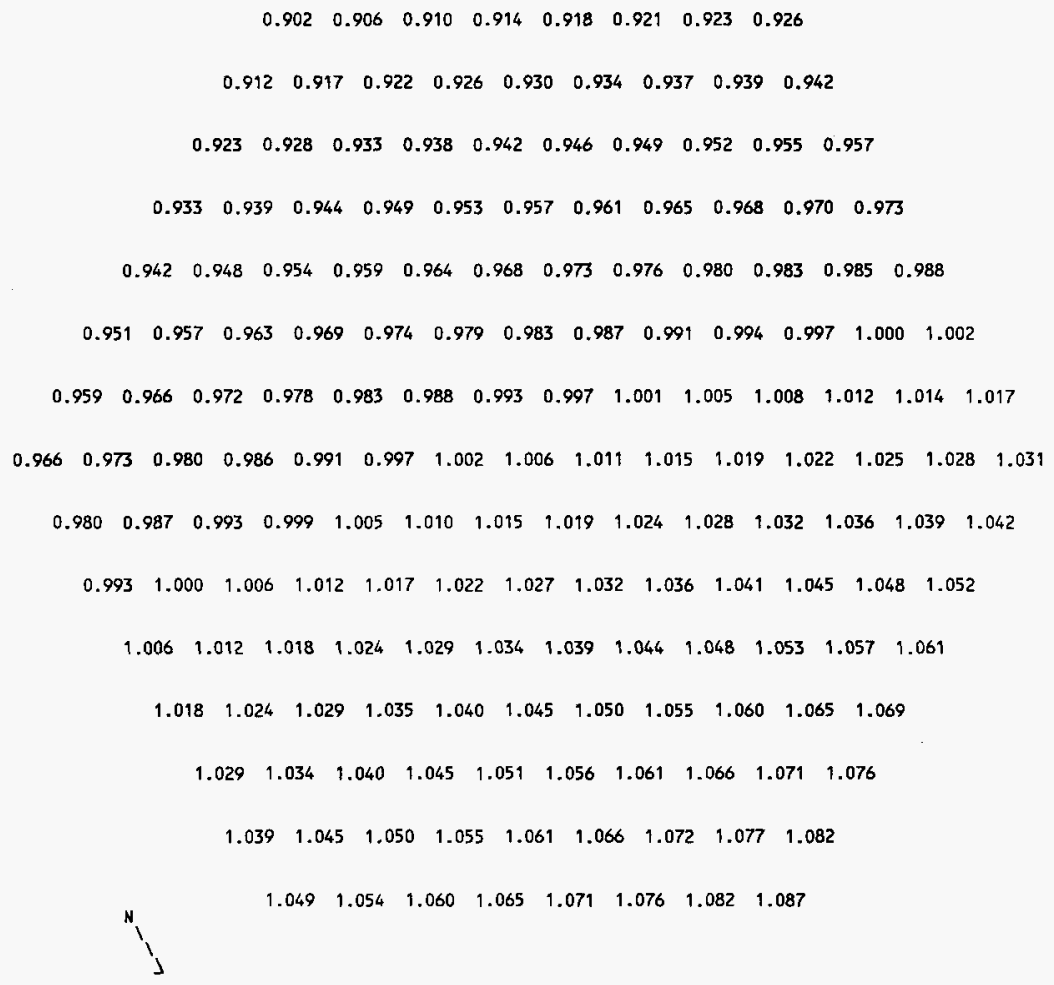




\section{WHC-SD-FF-ANAL-009 REV 0}

Table B10A-4.6. Fission Power Distribution by Pin in MFA-2 at BOC 1OA-4

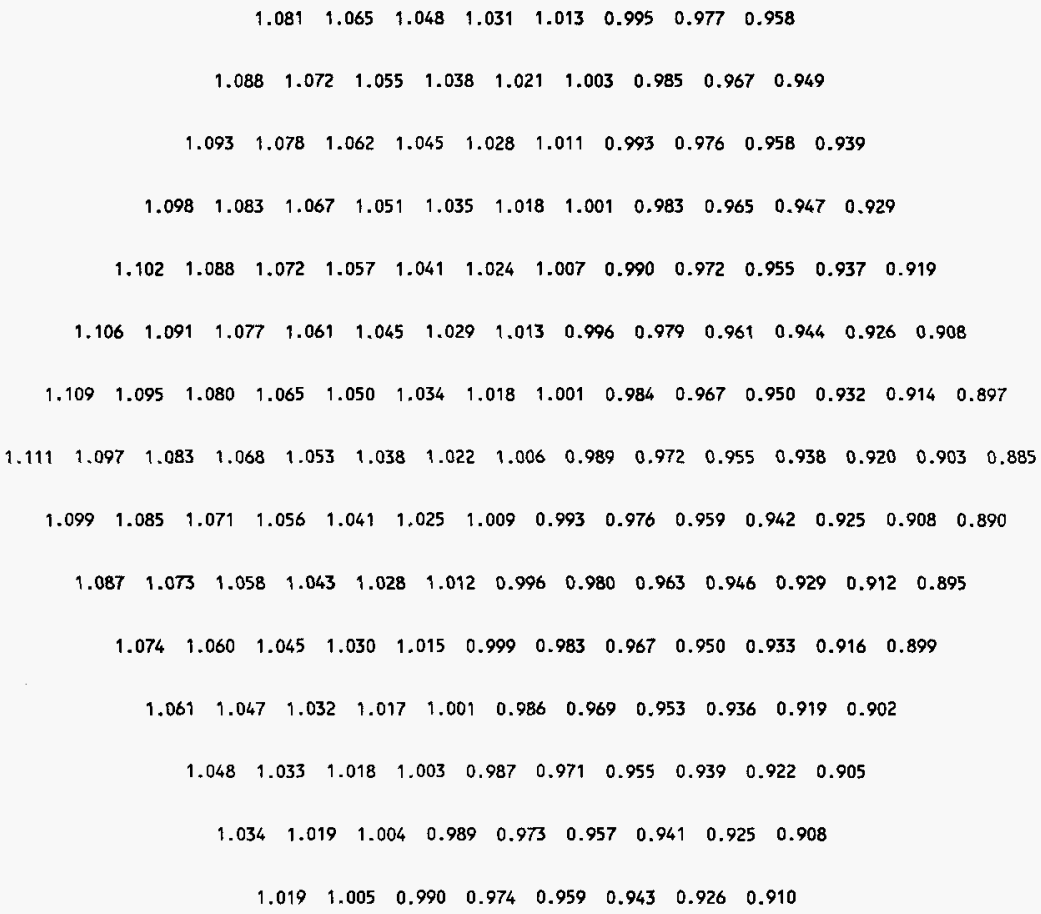


WHC-SD-FF-ANAL-009 REV 0

Table B10A-4.7. Total Fiux Distribution by Pin in MFA-1 at BOC 10A-4

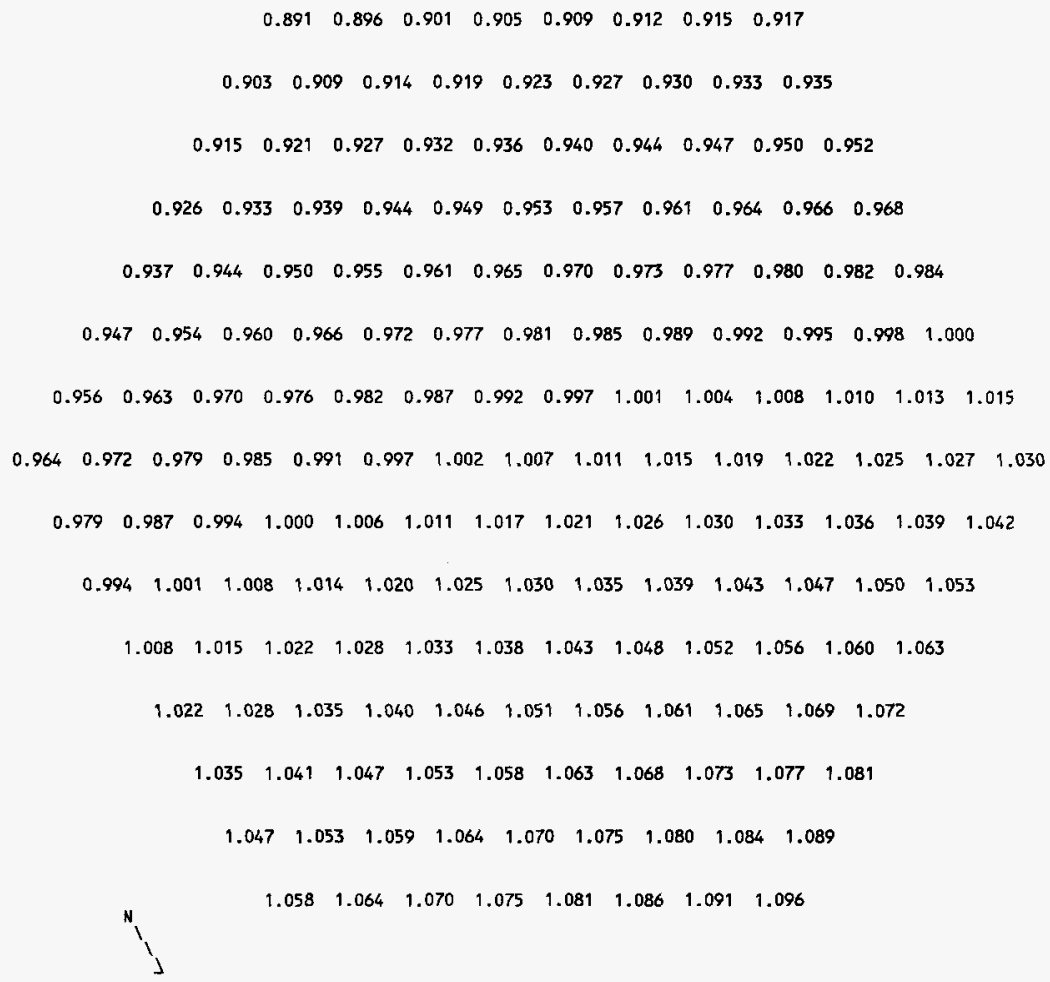


WHC-SD-FF-ANAL-009 REV 0

Table BlOA-4.8. Total Flux Distribution by Pin in MFA-2 at BOC 10A-4

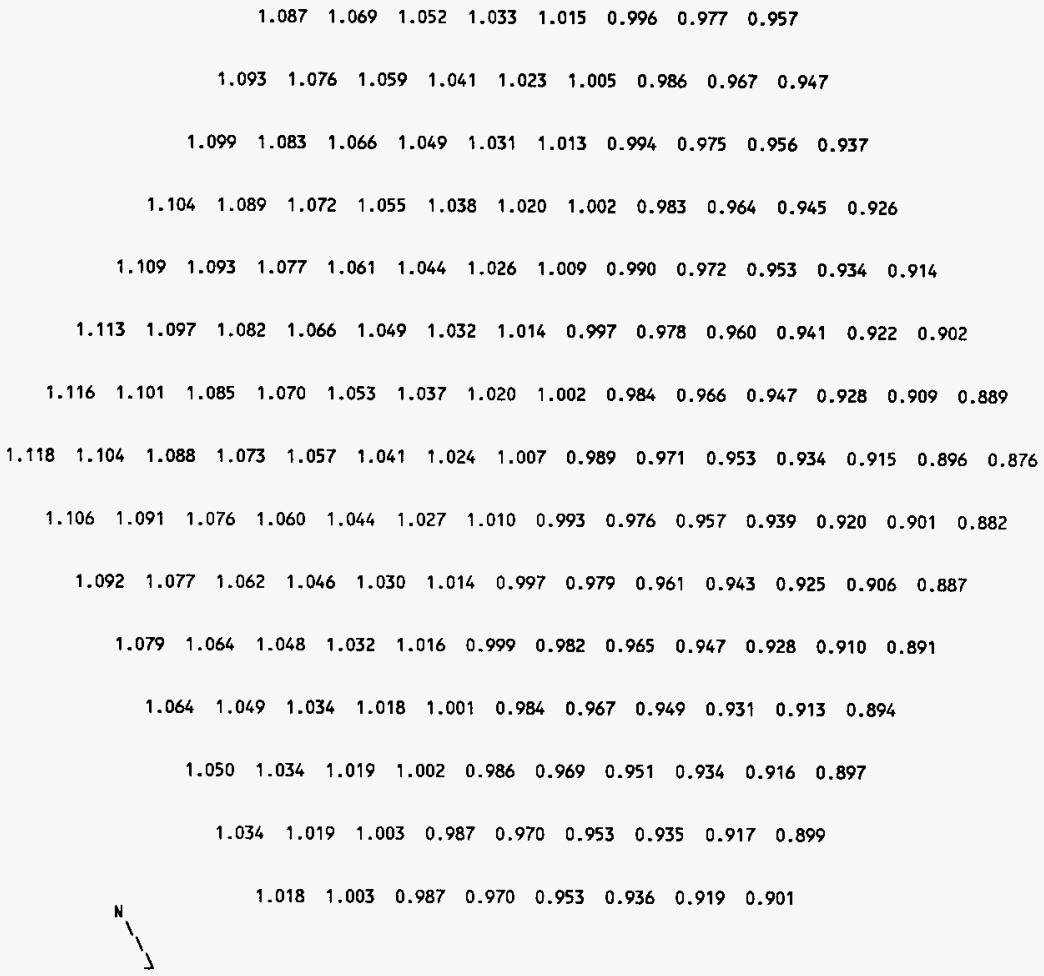


Table BlOA-4.9. MFA-1 and MFA-2 Duct Wall Flux Greater Than $0.1 \mathrm{MeV}$ at $\mathrm{BOC}$ 10A-4

$\begin{array}{ccc}\text { SIDE } & \begin{array}{c}\text { FLUX }>0.1 \\ \text { MFA-1 }\end{array} & \begin{array}{c}\text { MEV }\left(\mathrm{n} / \mathrm{cm}^{2}-\mathrm{sec}\right) \\ \text { MFA-2 }\end{array} \\ \text { E } & 1.8694 \mathrm{E}+15 & 2.1715 \mathrm{E}+15 \\ \text { SE } & 1.6702 \mathrm{E}+15 & 2.2491 \mathrm{E}+15 \\ \text { SW } & 1.6019 \mathrm{E}+15 & 2.0399 \mathrm{E}+15 \\ \text { W } & 1.7619 \mathrm{E}+15 & 1.7879 \mathrm{E}+15 \\ \text { NW } & 1.9794 \mathrm{E}+15 & 1.7228 \mathrm{E}+15 \\ \text { NE } & 2.0353 \mathrm{E}+15 & 1.8899 \mathrm{E}+15\end{array}$

Table B10A-4.10. Assembly outlet Temperatures and Flow Rates at BOC 10A-4

\begin{tabular}{lcc}
\multicolumn{3}{c}{ MEASURED } \\
CORE & OUTLET TEMP. & FLOW RATE \\
POS. & (DEG F) & (LB/H) \\
1506 & 1029 & 114940 \\
1404 & 914 & 225440 \\
1405 & 889 & 208130 \\
1505 & 882 & 208130 \\
1507 & 872 & 208130 \\
1606 & 900 & 170680 \\
1607 & 869 & 170680 \\
2507 & 1063 & 114940 \\
2405 & 946 & 192410 \\
2506 & 901 & 197920 \\
2508 & 931 & 208130 \\
2607 & 926 & 163690 \\
2608 & 905 & 170680 \\
2609 & 914 & 170680
\end{tabular}


Table E10A-4.1. Fission Power Generated in MFA-1, MFA-2 and Neighboring Assemblies at EOC 10A-4

\begin{tabular}{lllrl} 
CORE & $* * * * * *$ & \multicolumn{2}{c}{ POWER IN MEGAWATTS } & $* * * * * *$ \\
POS. & BELOW CORE & IN CORE & ABOVE CORE & TOTAL PWR \\
1506 & $3.241 \mathrm{E}-02$ & $3.799 \mathrm{E}+00$ & $2.518 \mathrm{E}-02$ & $3.857 \mathrm{E}+00$ \\
1404 & $8.461 \mathrm{E}-02$ & $4.559 \mathrm{E}+00$ & $5.420 \mathrm{E}-02$ & $4.698 \mathrm{E}+00$ \\
1405 & $9.766 \mathrm{E}-03$ & $3.786 \mathrm{E}+00$ & $8.299 \mathrm{E}-03$ & $3.804 \mathrm{E}+00$ \\
1505 & $1.127 \mathrm{E}-02$ & $3.577 \mathrm{E}+00$ & $8.230 \mathrm{E}-03$ & $3.597 \mathrm{E}+00$ \\
1507 & $9.733 \mathrm{E}-03$ & $3.213 \mathrm{E}+00$ & $8.314 \mathrm{E}-03$ & $3.231 \mathrm{E}+00$ \\
1606 & $6.404 \mathrm{E}-03$ & $3.447 \mathrm{E}+00$ & $5.535 \mathrm{E}-03$ & $3.459 \mathrm{E}+00$ \\
1607 & $9.048 \mathrm{E}-03$ & $2.810 \mathrm{E}+00$ & $7.536 \mathrm{E}-03$ & $2.827 \mathrm{E}+00$ \\
2507 & $3.402 \mathrm{E}-02$ & $4.044 \mathrm{E}+00$ & $2.767 \mathrm{E}-02$ & $4.106 \mathrm{E}+00$ \\
2405 & $7.395 \mathrm{E}-02$ & $4.282 \mathrm{E}+00$ & $4.958 \mathrm{E}-02$ & $4.406 \mathrm{E}+00$ \\
2506 & $1.148 \mathrm{E}-02$ & $3.770 \mathrm{E}+00$ & $9.332 \mathrm{E}-03$ & $3.791 \mathrm{E}+00$ \\
2508 & $1.138 \mathrm{E}-02$ & $4.459 \mathrm{E}+00$ & $9.609 \mathrm{E}-03$ & $4.480 \mathrm{E}+00$ \\
2607 & $7.988 \mathrm{E}-03$ & $3.483 \mathrm{E}+00$ & $6.782 \mathrm{E}-03$ & $3.498 \mathrm{E}+00$ \\
2608 & $5.315 \mathrm{E}-03$ & $3.370 \mathrm{E}+00$ & $4.923 \mathrm{E}-03$ & $3.381 \mathrm{E}+00$ \\
2609 & $1.072 \mathrm{E}-02$ & $3.522 \mathrm{E}+00$ & $8.034 \mathrm{E}-03$ & $3.541 \mathrm{E}+00$
\end{tabular}

Table E1OA-4.2. Assembly Averaged Total Flux for MFA-1 and MFA-2 at EOC 10A-4

$\begin{array}{ccc} & \text { Core } & \text { Total Flux } \\ \text { Assembly } & \text { Pos. } & \begin{array}{c}\left.\text { (n/cm }{ }^{2}-\mathrm{sec}\right) \\ \text { MFA-1 }\end{array} \\ \text { MFA-2 } & 2506 & 2.869 \mathrm{E}+15 \\ \text { MFA } & 3.051 \mathrm{E}+15\end{array}$


Table ElOA-4.3. Axial Distribution of Total Flux, Fast Flux and Power in MFA-1 at EOC 10A-4

$\begin{array}{rccc}z(\mathrm{~cm}) & \text { Total Flux } & \text { Flux }>\text { O.1 MeV } & \text { Power } \\ -97.78 & 2.6179 \mathrm{E}-02 & 1.3345 \mathrm{E}-02 & 0.0000 \mathrm{E}+00 \\ -92.78 & 5.6273 \mathrm{E}-02 & 2.5769 \mathrm{E}-02 & 0.0000 \mathrm{E}+00 \\ -87.78 & 9.0229 \mathrm{E}-02 & 4.1233 \mathrm{E}-02 & 0.0000 \mathrm{E}+00 \\ -82.78 & 1.3062 \mathrm{E}-01 & 6.1502 \mathrm{E}-02 & 0.0000 \mathrm{E}+00 \\ -77.78 & 1.8001 \mathrm{E}-01 & 8.8751 \mathrm{E}-02 & 0.0000 \mathrm{E}+00 \\ -72.15 & 2.3585 \mathrm{E}-01 & 1.2398 \mathrm{E}-01 & 0.0000 \mathrm{E}+00 \\ -65.90 & 2.9127 \mathrm{E}-01 & 1.6822 \mathrm{E}-01 & 0.0000 \mathrm{E}+00 \\ -60.35 & 3.4958 \mathrm{E}-01 & 2.2778 \mathrm{E}-01 & 3.3357 \mathrm{E}-02 \\ -55.49 & 4.2719 \mathrm{E}-01 & 3.0808 \mathrm{E}-01 & 4.2119 \mathrm{E}-02 \\ -50.62 & 5.1790 \mathrm{E}-01 & 4.1317 \mathrm{E}-01 & 5.5966 \mathrm{E}-02 \\ -47.17 & 5.9130 \mathrm{E}-01 & 5.1298 \mathrm{E}-01 & 7.2236 \mathrm{E}-02 \\ -43.58 & 6.6870 \mathrm{E}-01 & 6.2663 \mathrm{E}-01 & 7.1007 \mathrm{E}-01 \\ -38.45 & 7.8058 \mathrm{E}-01 & 7.6406 \mathrm{E}-01 & 7.9754 \mathrm{E}-01 \\ -33.32 & 8.8508 \mathrm{E}-01 & 8.8184 \mathrm{E}-01 & 8.9462 \mathrm{E}-01 \\ -28.20 & 9.7757 \mathrm{E}-01 & 9.8093 \mathrm{E}-01 & 9.7199 \mathrm{E}-01 \\ -23.07 & 1.0565 \mathrm{E}+00 & 1.0633 \mathrm{E}+00 & 1.0489 \mathrm{E}+00 \\ -17.94 & 1.1205 \mathrm{E}+00 & 1.1290 \mathrm{E}+00 & 1.1115 \mathrm{E}+00 \\ -12.82 & 1.1685 \mathrm{E}+00 & 1.1775 \mathrm{E}+00 & 1.1510 \mathrm{E}+00 \\ -7.69 & 1.2006 \mathrm{E}+00 & 1.2099 \mathrm{E}+00 & 1.1823 \mathrm{E}+00 \\ -2.56 & 1.2164 \mathrm{E}+00 & 1.2261 \mathrm{E}+00 & 1.1978 \mathrm{E}+00 \\ 2.56 & 1.2157 \mathrm{E}+00 & 1.2257 \mathrm{E}+00 & 1.1989 \mathrm{E}+00 \\ 7.69 & 1.1980 \mathrm{E}+00 & 1.2085 \mathrm{E}+00 & 1.1815 \mathrm{E}+00 \\ 12.82 & 1.1635 \mathrm{E}+00 & 1.1743 \mathrm{E}+00 & 1.1476 \mathrm{E}+00 \\ 17.94 & 1.1122 \mathrm{E}+00 & 1.1232 \mathrm{E}+00 & 1.1077 \mathrm{E}+00 \\ 23.07 & 1.0437 \mathrm{E}+00 & 1.0536 \mathrm{E}+00 & 1.0401 \mathrm{E}+00 \\ 28.20 & 9.5881 \mathrm{E}-01 & 9.6573 \mathrm{E}-01 & 9.5681 \mathrm{E}-01 \\ 33.32 & 8.5936 \mathrm{E}-01 & 8.6032 \mathrm{E}-01 & 8.7280 \mathrm{E}-01 \\ 38.45 & 7.4714 \mathrm{E}-01 & 7.3606 \mathrm{E}-01 & 7.6554 \mathrm{E}-01 \\ 43.58 & 6.2724 \mathrm{E}-01 & 5.9339 \mathrm{E}-01 & 6.6331 \mathrm{E}-01 \\ 47.17 & 5.4366 \mathrm{E}-01 & 4.7864 \mathrm{E}-01 & 6.2155 \mathrm{E}-02 \\ 50.62 & 4.6413 \mathrm{E}-01 & 3.7764 \mathrm{E}-01 & 4.5746 \mathrm{E}-02 \\ 55.49 & 3.6477 \mathrm{E}-01 & 2.7079 \mathrm{E}-01 & 3.1534 \mathrm{E}-02 \\ 60.35 & 2.7810 \mathrm{E}-01 & 1.8863 \mathrm{E}-01 & 2.2269 \mathrm{E}-02 \\ 64.98 & 2.2492 \mathrm{E}-01 & 1.4162 \mathrm{E}-01 & 0.0000 \mathrm{E}+00 \\ 69.39 & 1.9361 \mathrm{E}-01 & 1.1684 \mathrm{E}-01 & 0.0000 \mathrm{E}+00 \\ 76.46 & 1.4850 \mathrm{E}-01 & 8.3473 \mathrm{E}-02 & 0.0000 \mathrm{E}+00 \\ 86.80 & 9.7675 \mathrm{E}-02 & 5.0667 \mathrm{E}-02 & 0.0000 \mathrm{E}+00 \\ 98.14 & 5.6647 \mathrm{E}-02 & 2.7816 \mathrm{E}-02 & 0.0000 \mathrm{E}+00 \\ 110.00 & 2.4303 \mathrm{E}-02 & 1.2495 \mathrm{E}-02 & 0.0000 \mathrm{E}+00\end{array}$


Table El0A-4.4. Axial Distribution of Total Flux, Fast Flux and Power in MFA-2 at EOC $10 \mathrm{~A}-4$

$\begin{array}{rccc}\text { z }(\mathrm{cm}) & \text { Total Flux } & \text { FTux }>0.1 \text { MeV } & \text { Power } \\ -97.78 & 2.4099 \mathrm{E}-02 & 1.2324 \mathrm{E}-02 & 0.0000 \mathrm{E}+00 \\ -92.78 & 5.1768 \mathrm{E}-02 & 2.3786 \mathrm{E}-02 & 0.0000 \mathrm{E}+00 \\ -87.78 & 8.3038 \mathrm{E}-02 & 3.8064 \mathrm{E}-02 & 0.0000 \mathrm{E}+00 \\ -82.78 & 1.2041 \mathrm{E}-01 & 5.6826 \mathrm{E}-02 & 0.0000 \mathrm{E}+00 \\ -77.78 & 1.6662 \mathrm{E}-01 & 8.2190 \mathrm{E}-02 & 0.0000 \mathrm{E}+00 \\ -72.15 & 2.1997 \mathrm{E}-01 & 1.1533 \mathrm{E}-01 & 0.0000 \mathrm{E}+00 \\ -65.90 & 2.7501 \mathrm{E}-01 & 1.5801 \mathrm{E}-01 & 0.0000 \mathrm{E}+00 \\ -60.35 & 3.3448 \mathrm{E}-01 & 2.1636 \mathrm{E}-01 & 3.2407 \mathrm{E}-02 \\ -55.49 & 4.1279 \mathrm{E}-01 & 2.9572 \mathrm{E}-01 & 4.1384 \mathrm{E}-02 \\ -50.62 & 5.0478 \mathrm{E}-01 & 4.0091 \mathrm{E}-01 & 5.5481 \mathrm{E}-02 \\ -47.17 & 5.7957 \mathrm{E}-01 & 5.0173 \mathrm{E}-01 & 7.1958 \mathrm{E}-02 \\ -43.58 & 6.5863 \mathrm{E}-01 & 6.1665 \mathrm{E}-01 & 7.0285 \mathrm{E}-01 \\ -38.45 & 7.7316 \mathrm{E}-01 & 7.5659 \mathrm{E}-01 & 7.9120 \mathrm{E}-01 \\ -33.32 & 8.8034 \mathrm{E}-01 & 8.7714 \mathrm{E}-01 & 8.9029 \mathrm{E}-01 \\ -28.20 & 9.7536 \mathrm{E}-01 & 9.7901 \mathrm{E}-01 & 9.6944 \mathrm{E}-01 \\ -23.07 & 1.0568 \mathrm{E}+00 & 1.0642 \mathrm{E}+00 & 1.0486 \mathrm{E}+00 \\ -17.94 & 1.1232 \mathrm{E}+00 & 1.1322 \mathrm{E}+00 & 1.1136 \mathrm{E}+00 \\ -12.82 & 1.1732 \mathrm{E}+00 & 1.1828 \mathrm{E}+00 & 1.1549 \mathrm{E}+00 \\ -7.69 & 1.2067 \mathrm{E}+00 & 1.2164 \mathrm{E}+00 & 1.1876 \mathrm{E}+00 \\ -2.56 & 1.2228 \mathrm{E}+00 & 1.2329 \mathrm{E}+00 & 1.2033 \mathrm{E}+00 \\ 2.56 & 1.2215 \mathrm{E}+00 & 1.2320 \mathrm{E}+00 & 1.2037 \mathrm{E}+00 \\ 7.69 & 1.2027 \mathrm{E}+00 & 1.2136 \mathrm{E}+00 & 1.1852 \mathrm{E}+00 \\ 12.82 & 1.1667 \mathrm{E}+00 & 1.1777 \mathrm{E}+00 & 1.1499 \mathrm{E}+00 \\ 17.94 & 1.1137 \mathrm{E}+00 & 1.1246 \mathrm{E}+00 & 1.1085 \mathrm{E}+00 \\ 23.07 & 1.0436 \mathrm{E}+00 & 1.0531 \mathrm{E}+00 & 1.0395 \mathrm{E}+00 \\ 28.20 & 9.5740 \mathrm{E}-01 & 9.6365 \mathrm{E}-01 & 9.5492 \mathrm{E}-01 \\ 33.32 & 8.5680 \mathrm{E}-01 & 8.5695 \mathrm{E}-01 & 8.7025 \mathrm{E}-01 \\ 38.45 & 7.4381 \mathrm{E}-01 & 7.3177 \mathrm{E}-01 & 7.6302 \mathrm{E}-01 \\ 43.58 & 6.2360 \mathrm{E}-01 & 5.8861 \mathrm{E}-01 & 6.6320 \mathrm{E}-01 \\ 47.17 & 5.4045 \mathrm{E}-01 & 4.7374 \mathrm{E}-01 & 6.3544 \mathrm{E}-02 \\ 50.62 & 4.6149 \mathrm{E}-01 & 3.7284 \mathrm{E}-01 & 4.7019 \mathrm{E}-02 \\ 55.49 & 3.6344 \mathrm{E}-01 & 2.6709 \mathrm{E}-01 & 3.2682 \mathrm{E}-02 \\ 60.35 & 2.7806 \mathrm{E}-01 & 1.8643 \mathrm{E}-01 & 2.3303 \mathrm{E}-02 \\ 64.98 & 2.2528 \mathrm{E}-01 & 1.4009 \mathrm{E}-01 & 0.0000 \mathrm{E}+00 \\ 69.39 & 1.9351 \mathrm{E}-01 & 1.1529 \mathrm{E}-01 & 0.0000 \mathrm{E}+00 \\ 76.46 & 1.4766 \mathrm{E}-01 & 8.1858 \mathrm{E}-02 & 0.0000 \mathrm{E}+00 \\ 86.80 & 9.6566 \mathrm{E}-02 & 4.9259 \mathrm{E}-02 & 0.0000 \mathrm{E}+00 \\ 98.14 & 5.5677 \mathrm{E}-02 & 2.6783 \mathrm{E}-02 & 0.0000 \mathrm{E}+00 \\ 110.00 & 2.3593 \mathrm{E}-02 & 1.1851 \mathrm{E}-02 & 0.0000 \mathrm{E}+00\end{array}$


Table E10A-4.5. Fission Power Distribution by Pin in MFA-1 at EOC 10A-4

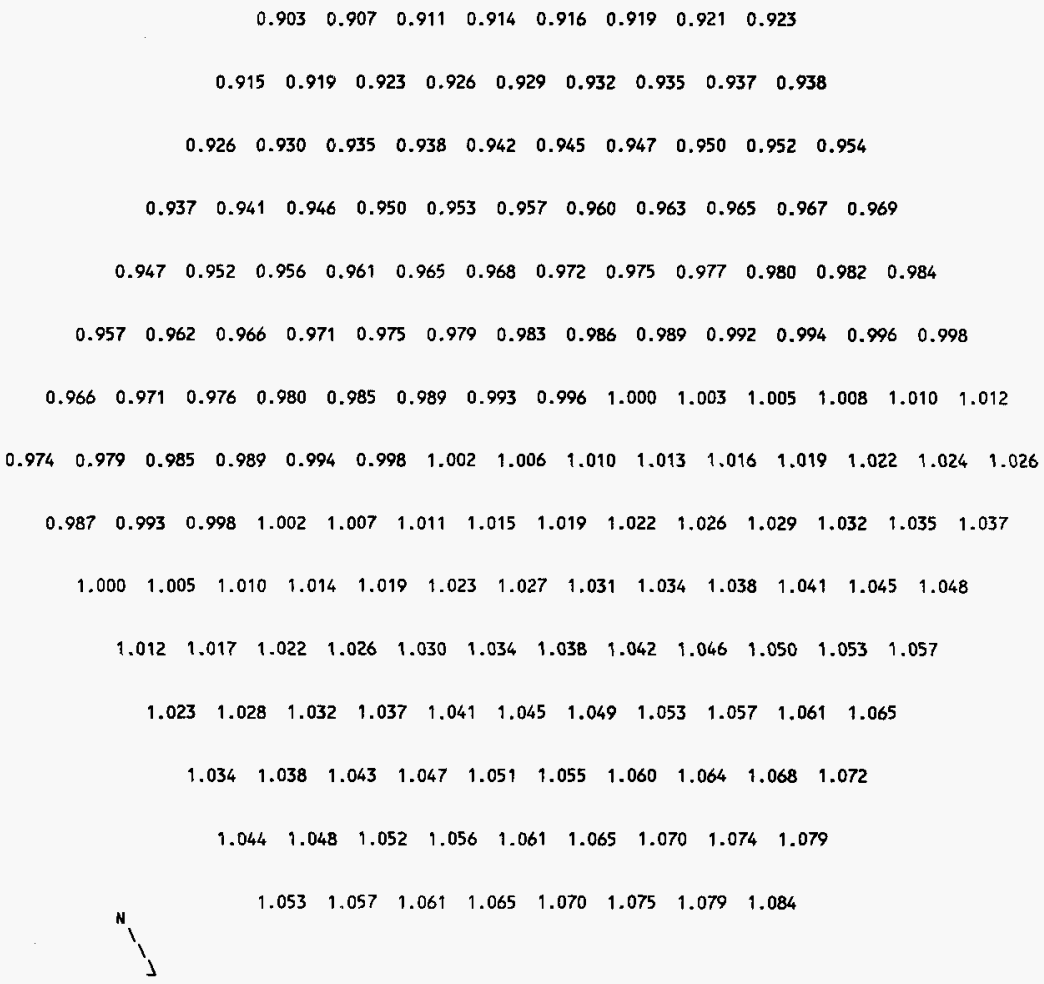




\section{WHC-SD-FF-ANAL-009 REV 0}

Table E1OA-4.6. Fission Power Distribution by Pin in MFA-2 at EOC 10A-4

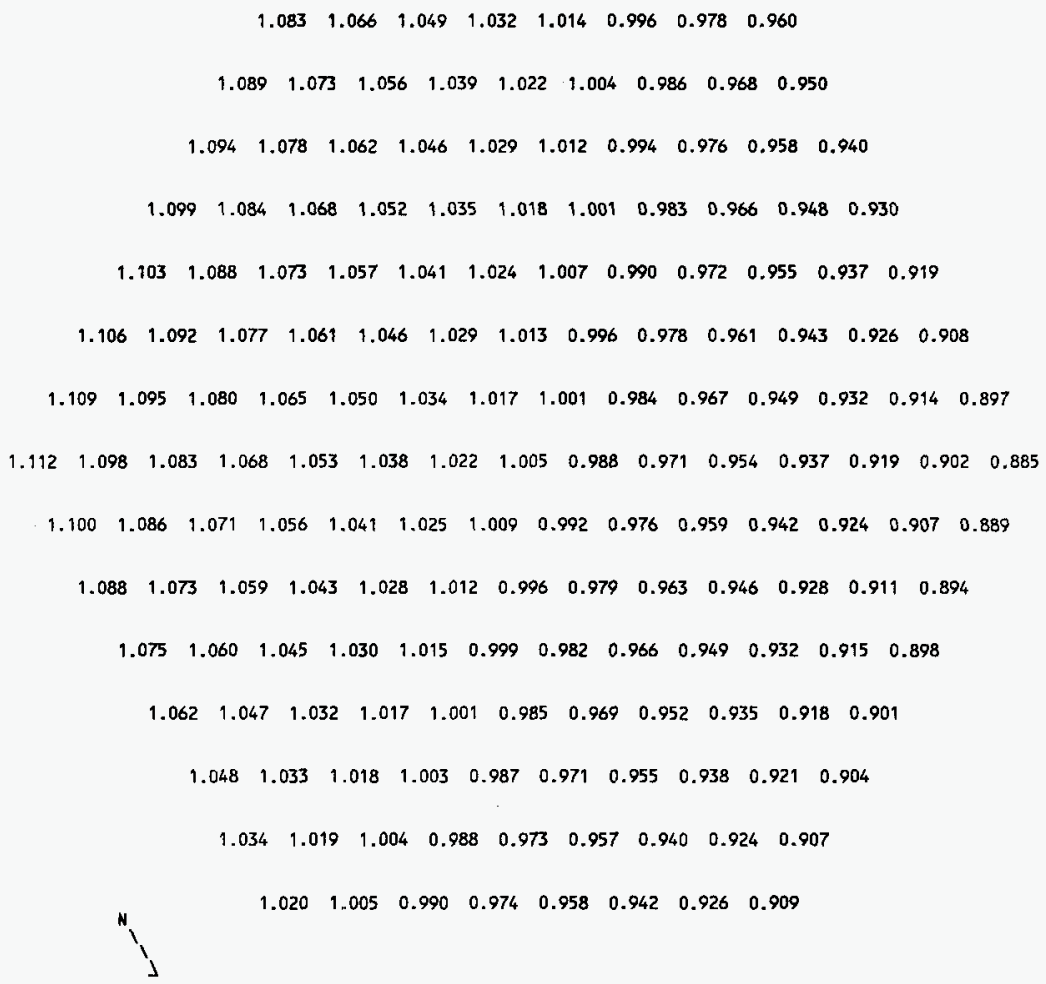


Table E10A-4.7. Total Flux Distribution by Pin in MFA-1 at EOC 10A-4

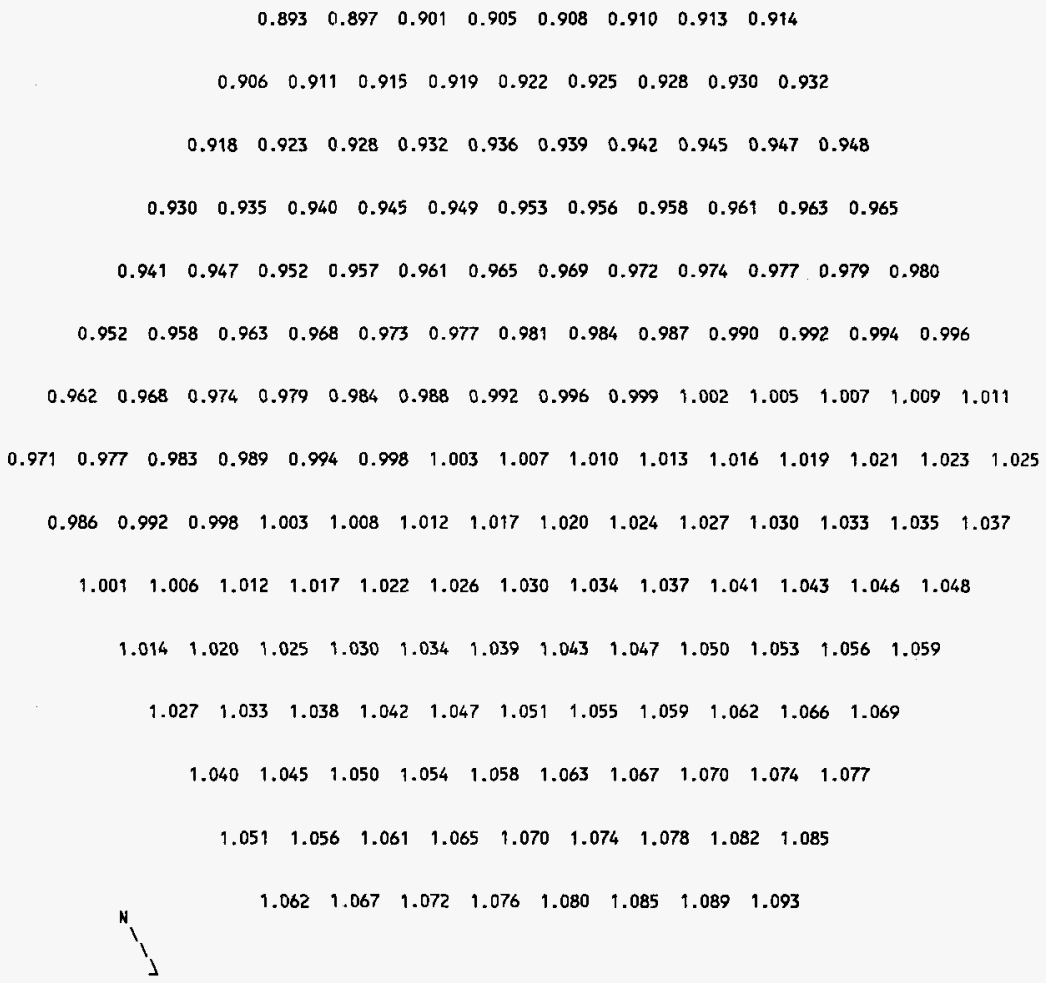




\section{WHC-SD-FF-ANAL-009 REV 0}

Table E1OA-4.8. Total Flux Distribution by Pin in MFA-2 at EOC 1OA-4

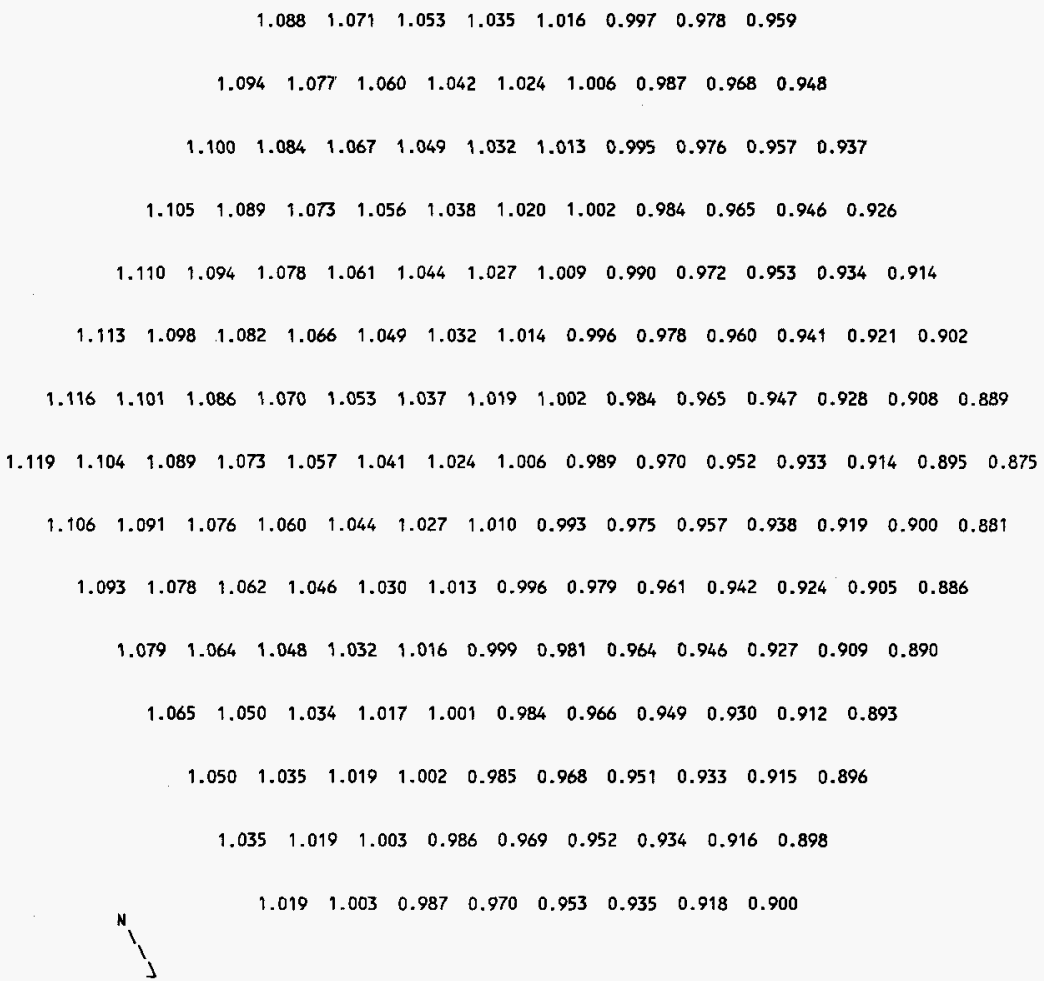


Table ElOA-4.9. MFA-1 and MFA-2 Duct Wall Flux Greater Than $0.1 \mathrm{MeV}$ at EOC $10 \mathrm{~A}-4$

$\begin{array}{ccc}\text { SIDE } & \text { FLUX }>0.1 & \text { MEV }\left(\mathrm{n} / \mathrm{cm}^{2}-\mathrm{sec}\right) \\ \text { MFA-1 } & \text { MFA-2 } \\ \text { E } & 1.8777 \mathrm{E}+15 & 2.1200 \mathrm{E}+15 \\ \text { SE } & 1.6743 \mathrm{E}+15 & 2.1949 \mathrm{E}+15 \\ \text { SW } & 1.5952 \mathrm{E}+15 & 1.9935 \mathrm{E}+15 \\ \text { W } & 1.7491 \mathrm{E}+15 & 1.7451 \mathrm{E}+15 \\ \text { NW } & 1.9651 \mathrm{E}+15 & 1.6780 \mathrm{E}+15 \\ \text { NE } & 2.0293 \mathrm{E}+15 & 1.8430 \mathrm{E}+15\end{array}$

Table ElOA-4.10. Assembly Outlet Temperatures and Flow Rates at EOC $10 \mathrm{~A}-4$

\begin{tabular}{|c|c|c|c|}
\hline $\begin{array}{l}\text { CORE } \\
\text { POS. } \\
1506 \\
1404 \\
1405 \\
1505 \\
1507 \\
1606 \\
1607 \\
2507 \\
2405 \\
2506 \\
2508 \\
2607 \\
2508\end{array}$ & $\begin{array}{c}\text { OUTLET T } \\
\text { MEASURED } \\
\text { 1027 } \\
911 \\
885 \\
884 \\
866 \\
899 \\
868 \\
1050 \\
938 \\
895 \\
920 \\
918 \\
899 \\
909\end{array}$ & $\begin{array}{c}\text { P. (DEG F) } \\
\text { CALCULATED } \\
1032 \\
908 \\
883 \\
870 \\
852 \\
898 \\
862 \\
1053 \\
930 \\
887 \\
915 \\
906 \\
894 \\
892\end{array}$ & $\begin{array}{c}\text { FLOW RATE } \\
\text { (LB/H) } \\
114940 \\
225440 \\
208130 \\
208130 \\
208130 \\
170680 \\
170680 \\
114940 \\
192410 \\
197920 \\
208130 \\
163690 \\
170680 \\
170680\end{array}$ \\
\hline
\end{tabular}


Table E10A-4.17. Composition of MFA-1 at EOC 10A-4

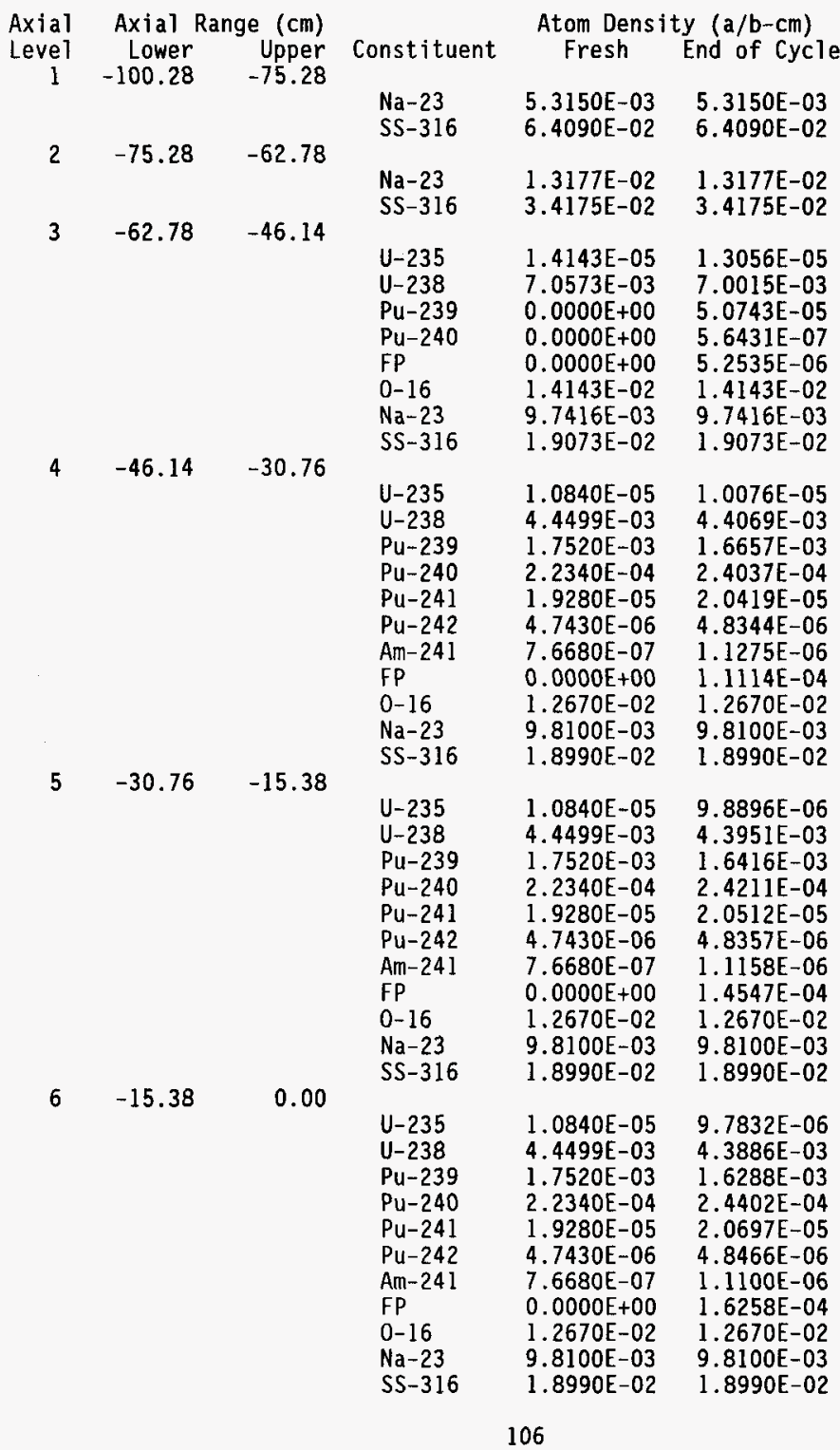


$\begin{array}{lll}7 & 0.00 & 15.38\end{array}$

$\begin{array}{lll}8 & 15.38 & 30.76\end{array}$

$\begin{array}{lll}\mathrm{U}-235 & 1.0840 \mathrm{E}-05 & 9.8089 \mathrm{E}-06 \\ \mathrm{U}-238 & 4.4499 \mathrm{E}-03 & 4.3902 \mathrm{E}-03 \\ \mathrm{Pu}-239 & 1.7520 \mathrm{E}-03 & 1.6319 \mathrm{E}-03 \\ \mathrm{PU}-240 & 2.2340 \mathrm{E}-04 & 2.4347 \mathrm{E}-04 \\ \mathrm{Pu}-241 & 1.9280 \mathrm{E}-05 & 2.0644 \mathrm{E}-05 \\ \mathrm{Pu}-242 & 4.7430 \mathrm{E}-06 & 4.8432 \mathrm{E}-06 \\ \mathrm{Am}-241 & 7.6680 \mathrm{E}-07 & 1.1112 \mathrm{E}-06 \\ \mathrm{FP} & 0.0000 \mathrm{E}+00 & 1.5852 \mathrm{E}-04 \\ \mathrm{O}-16 & 1.2670 \mathrm{E}-02 & 1.2670 \mathrm{E}-02 \\ \mathrm{Na}-23 & 9.8100 \mathrm{E}-03 & 9.8100 \mathrm{E}-03 \\ \mathrm{SS}-316 & 1.8990 \mathrm{E}-02 & 1.8990 \mathrm{E}-02\end{array}$

$\begin{array}{lll}\text { U-235 } & 1.0840 \mathrm{E}-05 & 9.9651 \mathrm{E}-06 \\ \mathrm{U}-238 & 4.4499 \mathrm{E}-03 & 4.3995 \mathrm{E}-03 \\ \mathrm{PU}-239 & 1.7520 \mathrm{E}-03 & 1.6502 \mathrm{E}-03 \\ \mathrm{Pu}-240 & 2.2340 \mathrm{E}-04 & 2.4053 \mathrm{E}-04 \\ \mathrm{Pu}-241 & 1.9280 \mathrm{E}-05 & 2.0361 \mathrm{E}-05 \\ \mathrm{PU}-242 & 4.7430 \mathrm{E}-06 & 4.8262 \mathrm{E}-06 \\ \mathrm{Am}-241 & 7.6680 \mathrm{E}-07 & 1.1193 \mathrm{E}-06 \\ \mathrm{FP} & 0.0000 \mathrm{E}+00 & 1.3413 \mathrm{E}-04 \\ \mathrm{O}-16 & 1.2670 \mathrm{E}-02 & 1.2670 \mathrm{E}-02 \\ \mathrm{Na}-23 & 9.8100 \mathrm{E}-03 & 9.8100 \mathrm{E}-03 \\ \mathrm{SS}-316 & 1.8990 \mathrm{E}-02 & 1.8990 \mathrm{E}-02\end{array}$

$\begin{array}{lll}9 & 30.76 \quad 46.14\end{array}$

$\begin{array}{lll}\text { U-235 } & 1.0840 \mathrm{E}-05 & 1.0190 \mathrm{E}-05 \\ \mathrm{U}-238 & 4.4499 \mathrm{E}-03 & 4.4134 \mathrm{E}-03 \\ \mathrm{Pu}-239 & 1.7520 \mathrm{E}-03 & 1.6783 \mathrm{E}-03 \\ \mathrm{Pu}-240 & 2.2340 \mathrm{E}-04 & 2.3749 \mathrm{E}-04 \\ \mathrm{Pu}-241 & 1.9280 \mathrm{E}-05 & 2.0125 \mathrm{E}-05 \\ \mathrm{Pu}-242 & 4.7430 \mathrm{E}-06 & 4.8156 \mathrm{E}-06 \\ \mathrm{Am}-241 & 7.6680 \mathrm{E}-07 & 1.1324 \mathrm{E}-06 \\ \mathrm{FP} & 0.0000 \mathrm{E}+00 & 9.5157 \mathrm{E}-05 \\ \mathrm{O}-16 & 1.2670 \mathrm{E}-02 & 1.2670 \mathrm{E}-02 \\ \mathrm{Na}-23 & 9.8100 \mathrm{E}-03 & 9.8100 \mathrm{E}-03 \\ \mathrm{SS}-316 & 1.8990 \mathrm{E}-02 & 1.8990 \mathrm{E}-02\end{array}$

$\begin{array}{lll}10 & 46.14 & 62.78\end{array}$

U-235 $\quad 1.4143 \mathrm{E}-05 \quad 1.3385 \mathrm{E}-05$

U-238 7.0573E-03 7.0174E-03

Pu-239 $\quad 0.0000 E+00 \quad 3.6387 E-05$

$\mathrm{Pu}-240 \quad 0.0000 \mathrm{E}+00 \quad 2.6913 \mathrm{E}-07$

FP $\quad 0.0000 E+00 \quad 3.7929 E-06$

$0-16 \quad 1.4143 \mathrm{E}-02 \quad 1.4143 \mathrm{E}-02$

$\mathrm{Na}-23 \quad 9.7416 \mathrm{E}-03 \quad 9.7416 \mathrm{E}-03$

SS-316 $1.9073 \mathrm{E}-02 \quad 1.9073 \mathrm{E}-02$

$\begin{array}{lll}11 & 62.78 & 116.00\end{array}$

$\begin{array}{lll}\mathrm{Na}-23 & 8.2400 \mathrm{E}-03 & 8.2400 \mathrm{E}-03 \\ \mathrm{SS}-316 & 2.5562 \mathrm{E}-02 & 2.5562 \mathrm{E}-02\end{array}$


Table E1OA-4.18. Composition of MFA-2 at EOC 1OA-4

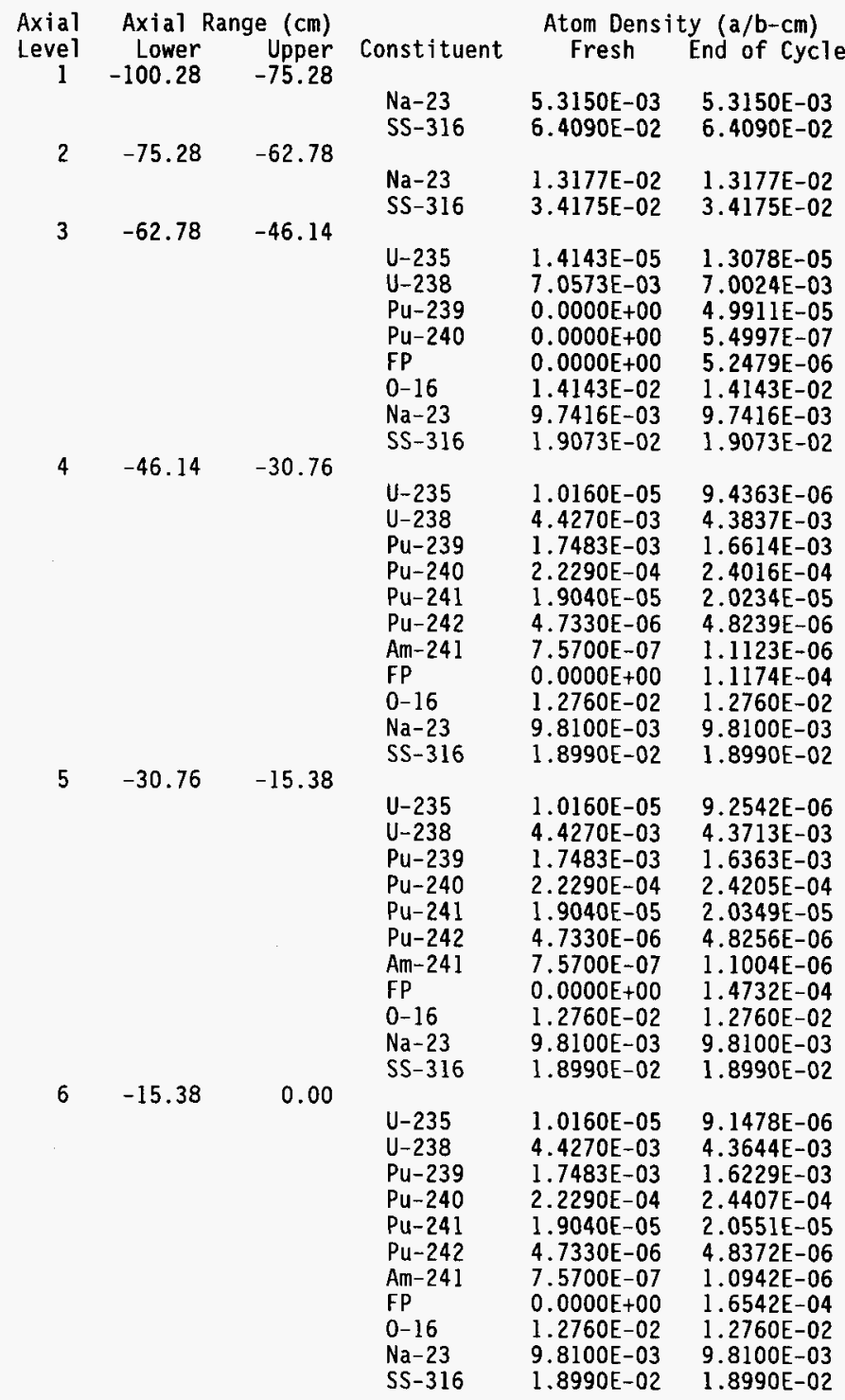


$\begin{array}{lll}7 & 0.00 \quad 15.38\end{array}$

$\begin{array}{lll}8 & 15.38 & 30.76\end{array}$

$\begin{array}{lll}\text { U-235 } & 1.0160 \mathrm{E}-05 & 9.1713 \mathrm{E}-06 \\ \mathrm{U}-238 & 4.4270 \mathrm{E}-03 & 4.3660 \mathrm{E}-03 \\ \mathrm{Pu}-239 & 1.7483 \mathrm{E}-03 & 1.6257 \mathrm{E}-03 \\ \mathrm{Pu}-240 & 2.2290 \mathrm{E}-04 & 2.4356 \mathrm{E}-04 \\ \mathrm{Pu}-241 & 1.9040 \mathrm{E}-05 & 2.0499 \mathrm{E}-05 \\ \mathrm{Pu}-242 & 4.7330 \mathrm{E}-06 & 4.8340 \mathrm{E}-06 \\ \mathrm{Am}-241 & 7.5700 \mathrm{E}-07 & 1.0953 \mathrm{E}-06 \\ \mathrm{FP} & 0.0000 \mathrm{E}+00 & 1.6162 \mathrm{E}-04 \\ \mathrm{O}-16 & 1.2760 \mathrm{E}-02 & 1.2760 \mathrm{E}-02 \\ \mathrm{Na}-23 & 9.8100 \mathrm{E}-03 & 9.8100 \mathrm{E}-03 \\ \text { SS-316 } & 1.8990 \mathrm{E}-02 & 1.8990 \mathrm{E}-02\end{array}$

$\begin{array}{lll}\text { U-235 } & 1.0160 \mathrm{E}-05 & 9.3168 \mathrm{E}-06 \\ \mathrm{U}-238 & 4.4270 \mathrm{E}-03 & 4.3753 \mathrm{E}-03 \\ \mathrm{Pu}-239 & 1.7483 \mathrm{E}-03 & 1.6440 \mathrm{E}-03 \\ \mathrm{PU}-240 & 2.2290 \mathrm{E}-04 & 2.4066 \mathrm{E}-04 \\ \mathrm{Pu}-241 & 1.9040 \mathrm{E}-05 & 2.0214 \mathrm{E}-05 \\ \mathrm{Pu}-242 & 4.7330 \mathrm{E}-06 & 4.8175 \mathrm{E}-06 \\ \mathrm{Am}-241 & 7.5700 \mathrm{E}-07 & 1.1034 \mathrm{E}-06 \\ \mathrm{FP} & 0.0000 \mathrm{E}+00 & 1.3721 \mathrm{E}-04 \\ 0-16 & 1.2760 \mathrm{E}-02 & 1.2760 \mathrm{E}-02 \\ \mathrm{Na}-23 & 9.8100 \mathrm{E}-03 & 9.8100 \mathrm{E}-03 \\ \mathrm{SS}-316 & 1.8990 \mathrm{E}-02 & 1.8990 \mathrm{E}-02\end{array}$

$\begin{array}{lll}9 & 30.76 \quad 46.14\end{array}$

$\begin{array}{lll}\mathrm{U}-235 & 1.0160 \mathrm{E}-05 & 9.5310 \mathrm{E}-06 \\ \mathrm{U}-238 & 4.4270 \mathrm{E}-03 & 4.3893 \mathrm{E}-03 \\ \mathrm{Pu}-239 & 1.7483 \mathrm{E}-03 & 1.6725 \mathrm{E}-03 \\ \mathrm{Pu}-240 & 2.2290 \mathrm{E}-04 & 2.3765 \mathrm{E}-04 \\ \mathrm{Pu}-241 & 1.9040 \mathrm{E}-05 & 1.9974 \mathrm{E}-05 \\ \mathrm{Pu}-242 & 4.7330 \mathrm{E}-06 & 4.8077 \mathrm{E}-06 \\ \mathrm{Am}-241 & 7.5700 \mathrm{E}-07 & 1.1166 \mathrm{E}-06 \\ \mathrm{FP} & 0.0000 \mathrm{E}+00 & 9.7751 \mathrm{E}-05 \\ \mathrm{O}-16 & 1.2760 \mathrm{E}-02 & 1.2760 \mathrm{E}-02 \\ \mathrm{Na}-23 & 9.8100 \mathrm{E}-03 & 9.8100 \mathrm{E}-03 \\ \mathrm{SS}-316 & 1.8990 \mathrm{E}-02 & 1.8990 \mathrm{E}-02\end{array}$

$\begin{array}{lll}10 & 46.14 & 62.78\end{array}$

$\begin{array}{lll}\text { U-235 } & 1.4143 \mathrm{E}-05 & 1.3355 \mathrm{E}-05 \\ \mathrm{U}-238 & 7.0573 \mathrm{E}-03 & 7.0158 \mathrm{E}-03 \\ \mathrm{Pu}-239 & 0.0000 \mathrm{E}+00 & 3.7793 \mathrm{E}-05 \\ \mathrm{Pu}-240 & 0.0000 \mathrm{E}+00 & 2.9713 \mathrm{E}-07 \\ \mathrm{FP} & 0.0000 \mathrm{E}+00 & 3.9889 \mathrm{E}-06 \\ 0-16 & 1.4143 \mathrm{E}-02 & 1.4143 \mathrm{E}-02 \\ \mathrm{Na}-23 & 9.7416 \mathrm{E}-03 & 9.7416 \mathrm{E}-03 \\ \mathrm{SS}-316 & 1.9073 \mathrm{E}-02 & 1.9073 \mathrm{E}-02\end{array}$

$11 \quad 62.78 \quad 116.00$

$\begin{array}{lll}\mathrm{Na}-23 & 8.2400 \mathrm{E}-03 & 8.2400 \mathrm{E}-03 \\ \mathrm{SS}-316 & 2.5562 \mathrm{E}-02 & 2.5562 \mathrm{E}-02\end{array}$


Table BlOB.1. Fission Power Generated in MFA-1, MFA-2 and Neighboring Assemblies at $\mathrm{BOC} 10 \mathrm{~B}$

\begin{tabular}{lcccc} 
CORE & $* * * * * *$ & \multicolumn{2}{c}{ POWER IN MEGAWATTS } & $* * * * * *$ \\
POS. & BELOW CORE & IN CORE & ABOVE CORE & TOTAL PWR \\
1506 & $3.473 \mathrm{E}-02$ & $3.674 \mathrm{E}+00$ & $2.243 \mathrm{E}-02$ & $3.731 \mathrm{E}+00$ \\
1404 & $8.999 \mathrm{E}-02$ & $4.349 \mathrm{E}+00$ & $4.727 \mathrm{E}-02$ & $4.486 \mathrm{E}+00$ \\
1405 & $0.000 \mathrm{E}+00$ & $6.471 \mathrm{E}+00$ & $0.000 \mathrm{E}+00$ & $6.471 \mathrm{E}+00$ \\
1505 & $6.642 \mathrm{E}-03$ & $4.040 \mathrm{E}+00$ & $4.411 \mathrm{E}-03$ & $4.051 \mathrm{E}+00$ \\
1507 & $1.025 \mathrm{E}-02$ & $3.107 \mathrm{E}+00$ & $7.320 \mathrm{E}-03$ & $3.124 \mathrm{E}+00$ \\
1606 & $6.841 \mathrm{E}-03$ & $3.304 \mathrm{E}+00$ & $4.704 \mathrm{E}-03$ & $3.316 \mathrm{E}+00$ \\
1607 & $9.531 \mathrm{E}-03$ & $2.732 \mathrm{E}+00$ & $6.635 \mathrm{E}-03$ & $2.748 \mathrm{E}+00$ \\
2507 & $3.646 \mathrm{E}-02$ & $4.003 \mathrm{E}+00$ & $2.503 \mathrm{E}-02$ & $4.065 \mathrm{E}+00$ \\
2405 & $1.008 \mathrm{E}-02$ & $4.134 \mathrm{E}+00$ & $7.467 \mathrm{E}-03$ & $4.151 \mathrm{E}+00$ \\
2506 & $6.915 \mathrm{E}-03$ & $4.386 \mathrm{E}+00$ & $5.308 \mathrm{E}-03$ & $4.398 \mathrm{E}+00$ \\
2508 & $1.223 \mathrm{E}-02$ & $4.393 \mathrm{E}+00$ & $8.597 \mathrm{E}-03$ & $4.414 \mathrm{E}+00$ \\
2607 & $8.402 \mathrm{E}-03$ & $3.401 \mathrm{E}+00$ & $6.054 \mathrm{E}-03$ & $3.415 \mathrm{E}+00$ \\
2608 & $5.672 \mathrm{E}-03$ & $3.352 \mathrm{E}+00$ & $4.515 \mathrm{E}-03$ & $3.362 \mathrm{E}+00$ \\
2609 & $6.230 \mathrm{E}-03$ & $4.224 \mathrm{E}+00$ & $4.945 \mathrm{E}-03$ & $4.235 \mathrm{E}+00$
\end{tabular}

Table B10B.2. Assembly Averaged Total Flux for MFA-1 and MFA-2 at BOC $10 \mathrm{~B}$

$\begin{array}{ccc} & \text { Core } & \text { Total Flux } \\ \text { Assembly } & \text { Pos. } & \left(\mathrm{n} / \mathrm{cm}^{2}-\mathrm{sec}\right) \\ \text { MFA-1 } & 1506 & 2.791 \mathrm{E}+15 \\ \text { MFA-2 } & 2507 & 3.016 \mathrm{E}+15\end{array}$


Table B10B.3. Axial Distribution of Total Flux, Fast Flux and Power in MFA-1 at $\mathrm{BOC} 10 \mathrm{~B}$

$\begin{array}{rccc}z(\mathrm{~cm}) & \text { Total Flux } & \text { Flux }>0.1 \mathrm{MeV} & \text { Power } \\ -97.78 & 2.8660 \mathrm{E}-02 & 1.4202 \mathrm{E}-02 & 0.0000 \mathrm{E}+00 \\ -92.78 & 6.1544 \mathrm{E}-02 & 2.7429 \mathrm{E}-02 & 0.0000 \mathrm{E}+00 \\ -87.78 & 9.8613 \mathrm{E}-02 & 4.3911 \mathrm{E}-02 & 0.0000 \mathrm{E}+00 \\ -82.78 & 1.4263 \mathrm{E}-01 & 6.5541 \mathrm{E}-02 & 0.0000 \mathrm{E}+00 \\ -77.78 & 1.9637 \mathrm{E}-01 & 9.4660 \mathrm{E}-02 & 0.0000 \mathrm{E}+00 \\ -72.15 & 2.5706 \mathrm{E}-01 & 1.3238 \mathrm{E}-01 & 0.0000 \mathrm{E}+00 \\ -65.90 & 3.1736 \mathrm{E}-01 & 1.8002 \mathrm{E}-01 & 0.0000 \mathrm{E}+00 \\ -60.35 & 3.8094 \mathrm{E}-01 & 2.4458 \mathrm{E}-01 & 3.6638 \mathrm{E}-02 \\ -55.49 & 4.6530 \mathrm{E}-01 & 3.3192 \mathrm{E}-01 & 4.6500 \mathrm{E}-02 \\ -50.62 & 5.6352 \mathrm{E}-01 & 4.4652 \mathrm{E}-01 & 6.2213 \mathrm{E}-02 \\ -47.17 & 6.4245 \mathrm{E}-01 & 5.5513 \mathrm{E}-01 & 8.0727 \mathrm{E}-02 \\ -43.58 & 7.2536 \mathrm{E}-01 & 6.7808 \mathrm{E}-01 & 7.6944 \mathrm{E}-01 \\ -38.45 & 8.4374 \mathrm{E}-01 & 8.2492 \mathrm{E}-01 & 8.6192 \mathrm{E}-01 \\ -33.32 & 9.5221 \mathrm{E}-01 & 9.4819 \mathrm{E}-01 & 9.6281 \mathrm{E}-01 \\ -28.20 & 1.0454 \mathrm{E}+00 & 1.0490 \mathrm{E}+00 & 1.0401 \mathrm{E}+00 \\ -23.07 & 1.1212 \mathrm{E}+00 & 1.1289 \mathrm{E}+00 & 1.1140 \mathrm{E}+00 \\ -17.94 & 1.1782 \mathrm{E}+00 & 1.1879 \mathrm{E}+00 & 1.1697 \mathrm{E}+00 \\ -12.82 & 1.2154 \mathrm{E}+00 & 1.2259 \mathrm{E}+00 & 1.1982 \mathrm{E}+00 \\ -7.69 & 1.2331 \mathrm{E}+00 & 1.2439 \mathrm{E}+00 & 1.2153 \mathrm{E}+00 \\ -2.56 & 1.2317 \mathrm{E}+00 & 1.2427 \mathrm{E}+00 & 1.2136 \mathrm{E}+00 \\ 2.56 & 1.2121 \mathrm{E}+00 & 1.2232 \mathrm{E}+00 & 1.1960 \mathrm{E}+00 \\ 7.69 & 1.1757 \mathrm{E}+00 & 1.1869 \mathrm{E}+00 & 1.1600 \mathrm{E}+00 \\ 12.82 & 1.1239 \mathrm{E}+00 & 1.1350 \mathrm{E}+00 & 1.1090 \mathrm{E}+00 \\ 17.94 & 1.0580 \mathrm{E}+00 & 1.0687 \mathrm{E}+00 & 1.0540 \mathrm{E}+00 \\ 23.07 & 9.7848 \mathrm{E}-01 & 9.8763 \mathrm{E}-01 & 9.7527 \mathrm{E}-01 \\ 28.20 & 8.8675 \mathrm{E}-01 & 8.9271 \mathrm{E}-01 & 8.8479 \mathrm{E}-01 \\ 33.32 & 7.8459 \mathrm{E}-01 & 7.8479 \mathrm{E}-01 & 7.9642 \mathrm{E}-01 \\ 38.45 & 6.7410 \mathrm{E}-01 & 6.6317 \mathrm{E}-01 & 6.8965 \mathrm{E}-01 \\ 43.58 & 5.6003 \mathrm{E}-01 & 5.2841 \mathrm{E}-01 & 5.8982 \mathrm{E}-01 \\ 47.17 & 4.8402 \mathrm{E}-01 & 4.2513 \mathrm{E}-01 & 5.7224 \mathrm{E}-02 \\ 50.62 & 4.1383 \mathrm{E}-01 & 3.3603 \mathrm{E}-01 & 4.2069 \mathrm{E}-02 \\ 55.49 & 3.2800 \mathrm{E}-01 & 2.4309 \mathrm{E}-01 & 2.9073 \mathrm{E}-02 \\ 60.35 & 2.5277 \mathrm{E}-01 & 1.7129 \mathrm{E}-01 & 2.0592 \mathrm{E}-02 \\ 64.98 & 2.0457 \mathrm{E}-01 & 1.2864 \mathrm{E}-01 & 0.0000 \mathrm{E}+00 \\ 69.39 & 1.7565 \mathrm{E}-01 & 1.0560 \mathrm{E}-01 & 0.0000 \mathrm{E}+00 \\ 76.46 & 1.3414 \mathrm{E}-01 & 7.4736 \mathrm{E}-02 & 0.0000 \mathrm{E}+00 \\ 86.80 & 8.8291 \mathrm{E}-02 & 4.5086 \mathrm{E}-02 & 0.0000 \mathrm{E}+00 \\ 98.14 & 5.1709 \mathrm{E}-02 & 2.4785 \mathrm{E}-02 & 0.0000 \mathrm{E}+00 \\ 110.00 & 2.2429 \mathrm{E}-02 & 1.1198 \mathrm{E}-02 & 0.0000 \mathrm{E}+00 \\ & & & \end{array}$


Table BlOB.4. Axial Distribution of Total Flux, Fast Flux and Power in MFA-2 at $B O C \quad 10 B$

$\begin{array}{rccc}\text { z }(\mathrm{cm}) & \text { Total Flux } & \text { Flux }>0.1 \text { MeV } & \text { Power } \\ -97.78 & 2.5586 \mathrm{E}-02 & 1.2970 \mathrm{E}-02 & 0.0000 \mathrm{E}+00 \\ -92.78 & 5.4933 \mathrm{E}-02 & 2.5033 \mathrm{E}-02 & 0.0000 \mathrm{E}+00 \\ -87.78 & 8.8076 \mathrm{E}-02 & 4.0058 \mathrm{E}-02 & 0.0000 \mathrm{E}+00 \\ -82.78 & 1.2768 \mathrm{E}-01 & 5.9806 \mathrm{E}-02 & 0.0000 \mathrm{E}+00 \\ -77.78 & 1.7671 \mathrm{E}-01 & 8.6514 \mathrm{E}-02 & 0.0000 \mathrm{E}+00 \\ -72.15 & 2.3360 \mathrm{E}-01 & 1.2149 \mathrm{E}-01 & 0.0000 \mathrm{E}+00 \\ -65.90 & 2.9288 \mathrm{E}-01 & 1.6681 \mathrm{E}-01 & 0.0000 \mathrm{E}+00 \\ -60.35 & 3.5697 \mathrm{E}-01 & 2.2888 \mathrm{E}-01 & 3.5079 \mathrm{E}-02 \\ -55.49 & 4.4044 \mathrm{E}-01 & 3.1310 \mathrm{E}-01 & 4.4821 \mathrm{E}-02 \\ -50.62 & 5.3782 \mathrm{E}-01 & 4.2488 \mathrm{E}-01 & 6.0040 \mathrm{E}-02 \\ -47.17 & 6.1702 \mathrm{E}-01 & 5.3230 \mathrm{E}-01 & 7.7936 \mathrm{E}-02 \\ -43.58 & 7.0086 \mathrm{E}-01 & 6.5493 \mathrm{E}-01 & 7.5091 \mathrm{E}-01 \\ -38.45 & 8.2168 \mathrm{E}-01 & 8.0305 \mathrm{E}-01 & 8.4138 \mathrm{E}-01 \\ -33.32 & 9.3305 \mathrm{E}-01 & 9.2889 \mathrm{E}-01 & 9.4359 \mathrm{E}-01 \\ -28.20 & 1.0295 \mathrm{E}+00 & 1.0331 \mathrm{E}+00 & 1.0232 \mathrm{E}+00 \\ -23.07 & 1.1091 \mathrm{E}+00 & 1.1170 \mathrm{E}+00 & 1.1004 \mathrm{E}+00 \\ -17.94 & 1.1702 \mathrm{E}+00 & 1.1803 \mathrm{E}+00 & 1.1603 \mathrm{E}+00 \\ -12.82 & 1.2119 \mathrm{E}+00 & 1.2229 \mathrm{E}+00 & 1.1930 \mathrm{E}+00 \\ -7.69 & 1.2340 \mathrm{E}+00 & 1.2453 \mathrm{E}+00 & 1.2145 \mathrm{E}+00 \\ -2.56 & 1.2367 \mathrm{E}+00 & 1.2482 \mathrm{E}+00 & 1.2170 \mathrm{E}+00 \\ 2.56 & 1.2205 \mathrm{E}+00 & 1.2322 \mathrm{E}+00 & 1.2027 \mathrm{E}+00 \\ 7.69 & 1.1867 \mathrm{E}+00 & 1.1982 \mathrm{E}+00 & 1.1694 \mathrm{E}+00 \\ 12.82 & 1.1365 \mathrm{E}+00 & 1.1476 \mathrm{E}+00 & 1.1200 \mathrm{E}+00 \\ 17.94 & 1.0711 \mathrm{E}+00 & 1.0815 \mathrm{E}+00 & 1.0659 \mathrm{E}+00 \\ 23.07 & 9.9147 \mathrm{E}-01 & 9.9994 \mathrm{E}-01 & 9.8731 \mathrm{E}-01 \\ 28.20 & 8.9903 \mathrm{E}-01 & 9.0411 \mathrm{E}-01 & 8.9641 \mathrm{E}-01 \\ 33.32 & 7.9575 \mathrm{E}-01 & 7.9495 \mathrm{E}-01 & 8.0787 \mathrm{E}-01 \\ 38.45 & 6.8386 \mathrm{E}-01 & 6.7202 \mathrm{E}-01 & 7.0127 \mathrm{E}-01 \\ 43.58 & 5.6799 \mathrm{E}-01 & 5.3567 \mathrm{E}-01 & 6.0483 \mathrm{E}-01 \\ 47.17 & 4.8951 \mathrm{E}-01 & 4.2874 \mathrm{E}-01 & 5.8736 \mathrm{E}-02 \\ 50.62 & 4.1562 \mathrm{E}-01 & 3.3536 \mathrm{E}-01 & 4.3165 \mathrm{E}-02 \\ 55.49 & 3.2514 \mathrm{E}-01 & 2.3852 \mathrm{E}-01 & 2.9747 \mathrm{E}-02 \\ 60.35 & 2.4675 \mathrm{E}-01 & 1.6520 \mathrm{E}-01 & 2.0985 \mathrm{E}-02 \\ 64.98 & 1.9826 \mathrm{E}-01 & 1.2316 \mathrm{E}-01 & 0.0000 \mathrm{E}+00 \\ 69.39 & 1.6952 \mathrm{E}-01 & 1.0089 \mathrm{E}-01 & 0.0000 \mathrm{E}+00 \\ 76.46 & 1.2865 \mathrm{E}-01 & 7.1169 \mathrm{E}-02 & 0.0000 \mathrm{E}+00 \\ 86.80 & 8.4008 \mathrm{E}-02 & 4.2658 \mathrm{E}-02 & 0.0000 \mathrm{E}+00 \\ 98.14 & 4.8631 \mathrm{E}-02 & 2.3205 \mathrm{E}-02 & 0.0000 \mathrm{E}+00 \\ 110.00 & 2.0726 \mathrm{E}-02 & 1.0302 \mathrm{E}-02 & 0.0000 \mathrm{E}+00\end{array}$


Table B10B.5. Fission Power Distribution by Pin in MFA-1 at BOC $10 \mathrm{~B}$

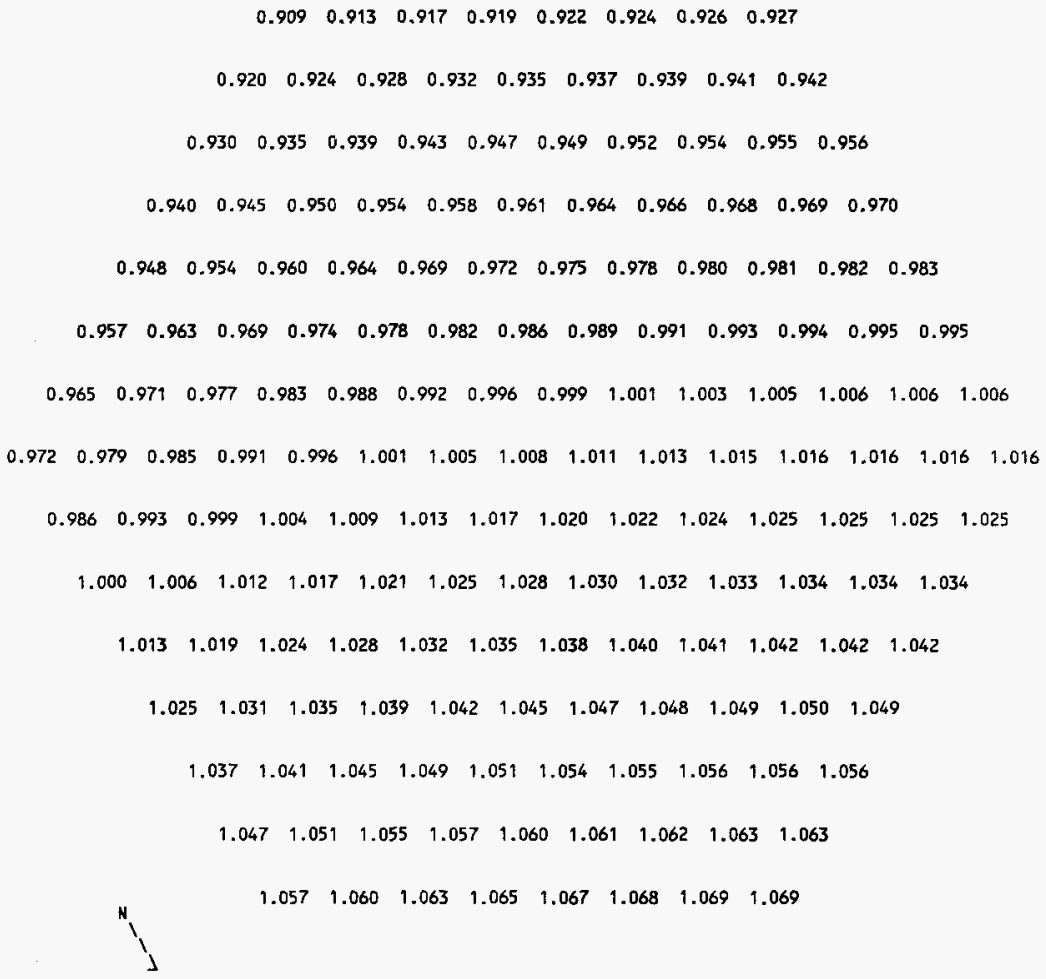


Table B10B.6. Fission Power Distribution by Pin in MFA-2 at BOC 10B

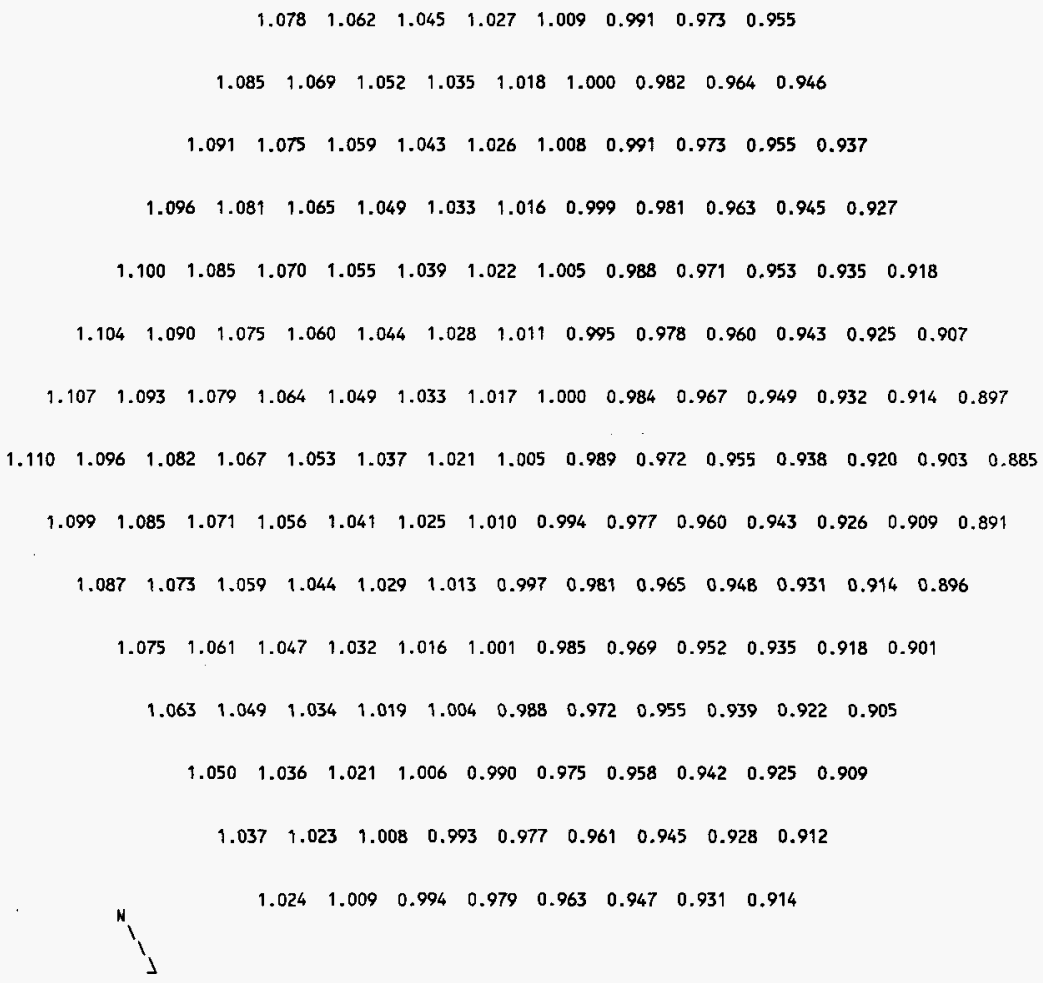




\section{WHC-SD-FF-ANAL-009 REV 0}

Table B10B.7. Total Flux Distribution by Pin in MFA-1 at BOC 10B

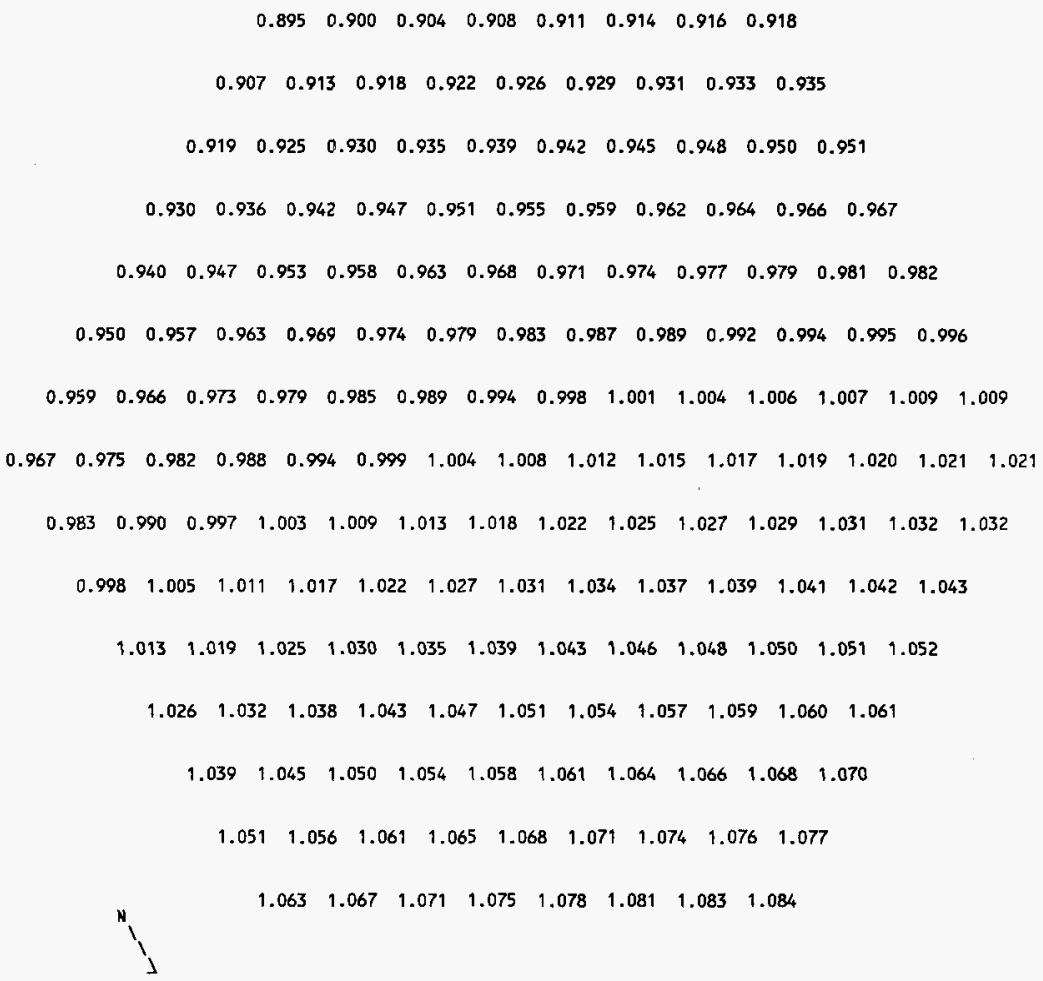


Table B10B.8. Total Flux Distribution by Pin in MFA-2 at BOC $10 B$

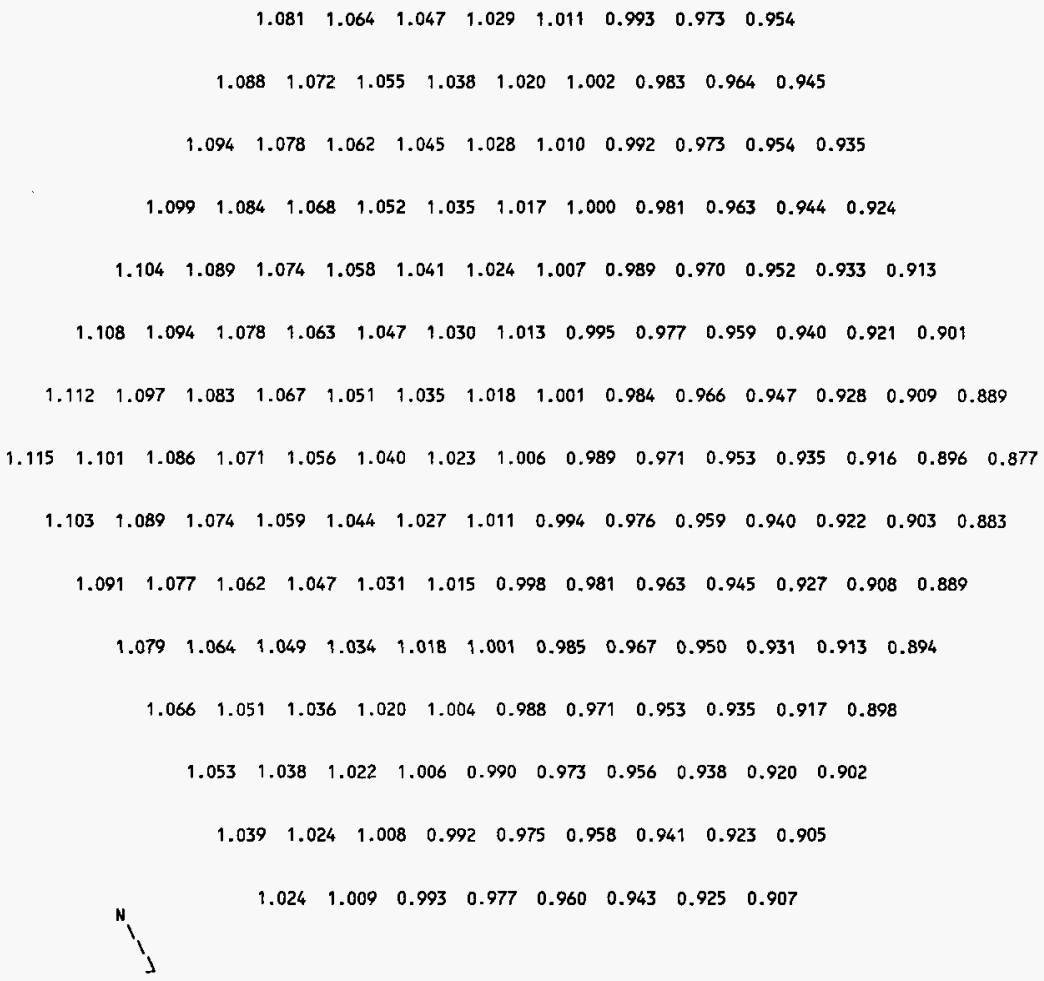


Table B10B.9. MFA-1 and MFA-2 Duct Wall Flux Greater Than $0.1 \mathrm{MeV}$ at $\mathrm{BOC} 10 \mathrm{~B}$

$\begin{array}{ccc}\text { SIDE } & \begin{array}{c}\text { FLUX }>0.1 \\ \text { MFA-1 }\end{array} & \begin{array}{c}\text { MEV } \\ \left.\text { ( } / \mathrm{cm}^{2}-\mathrm{sec}\right) \\ \text { MFA-2 }\end{array} \\ \text { E } & 1.8738 E+15 & 2.1211 \mathrm{E}+15 \\ \text { SE } & 1.6634 \mathrm{E}+15 & 2.1766 \mathrm{E}+15 \\ \text { SW } & 1.5985 \mathrm{E}+15 & 1.9890 \mathrm{E}+15 \\ \text { W } & 1.7757 \mathrm{E}+15 & 1.7425 \mathrm{E}+15 \\ \text { NW } & 2.0314 \mathrm{E}+15 & 1.6907 \mathrm{E}+15 \\ \text { NE } & 2.0435 E+15 & 1.8708 \mathrm{E}+15\end{array}$

Table B10B.10. Assembly Outlet Temperatures and Flow Rates at $B 0 C 10 B$

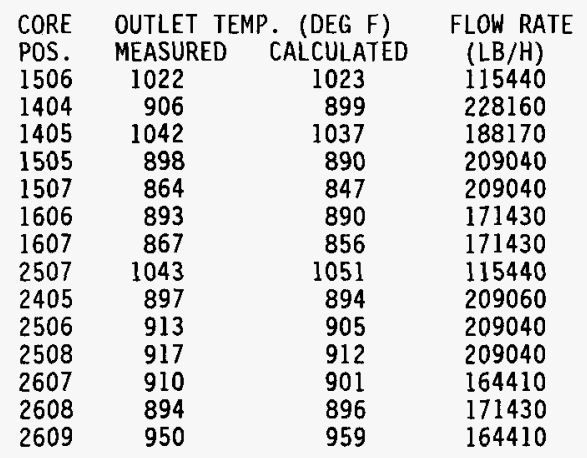


Table B10B.11. MFA-1 Sodium Subchanne1 Temperatures at Top of Core Elevation for BOC 10B

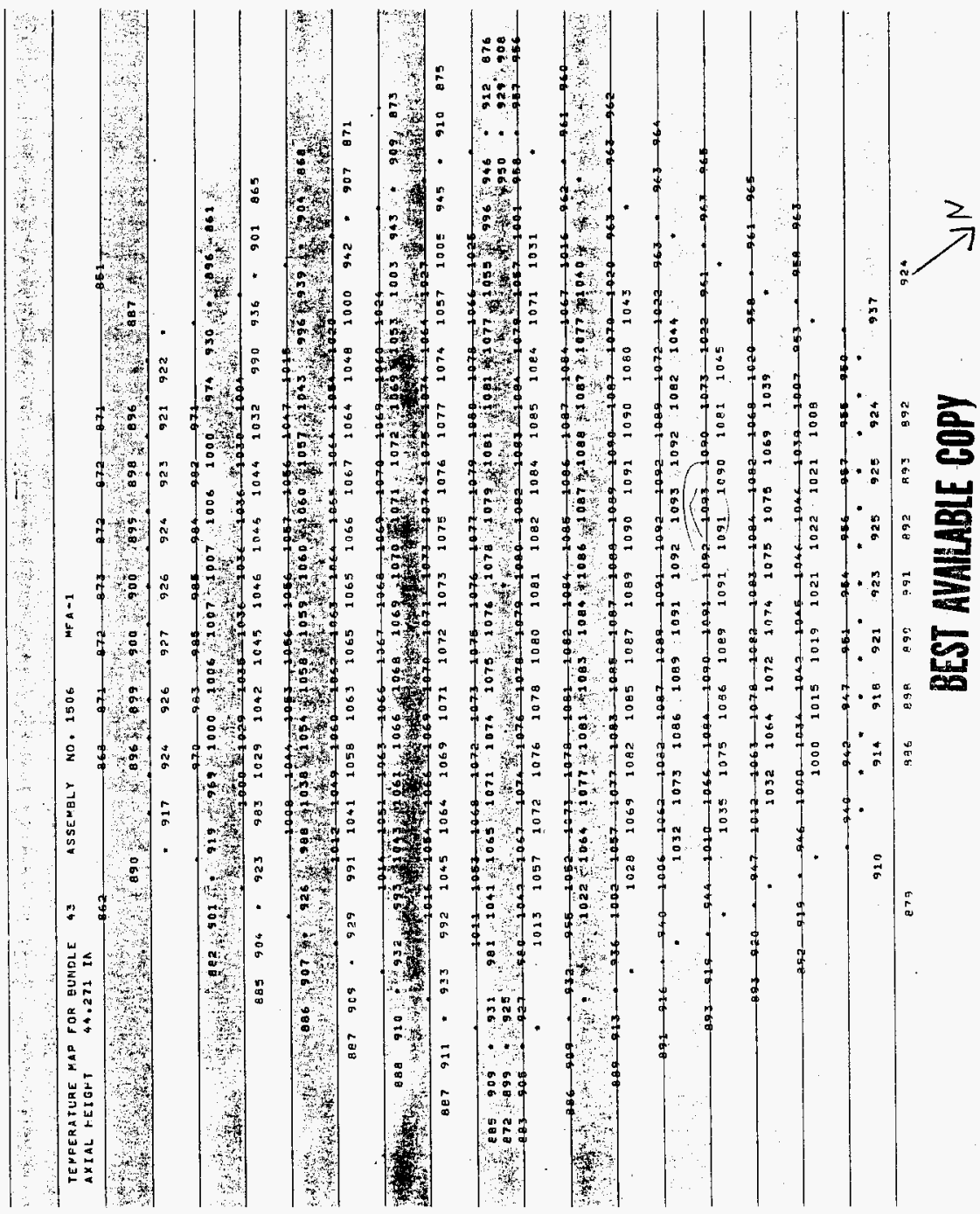


Table B10B.12. MFA-2 Sodium Subchannel Temperatures at Top of Core Elevation for BOC 10B

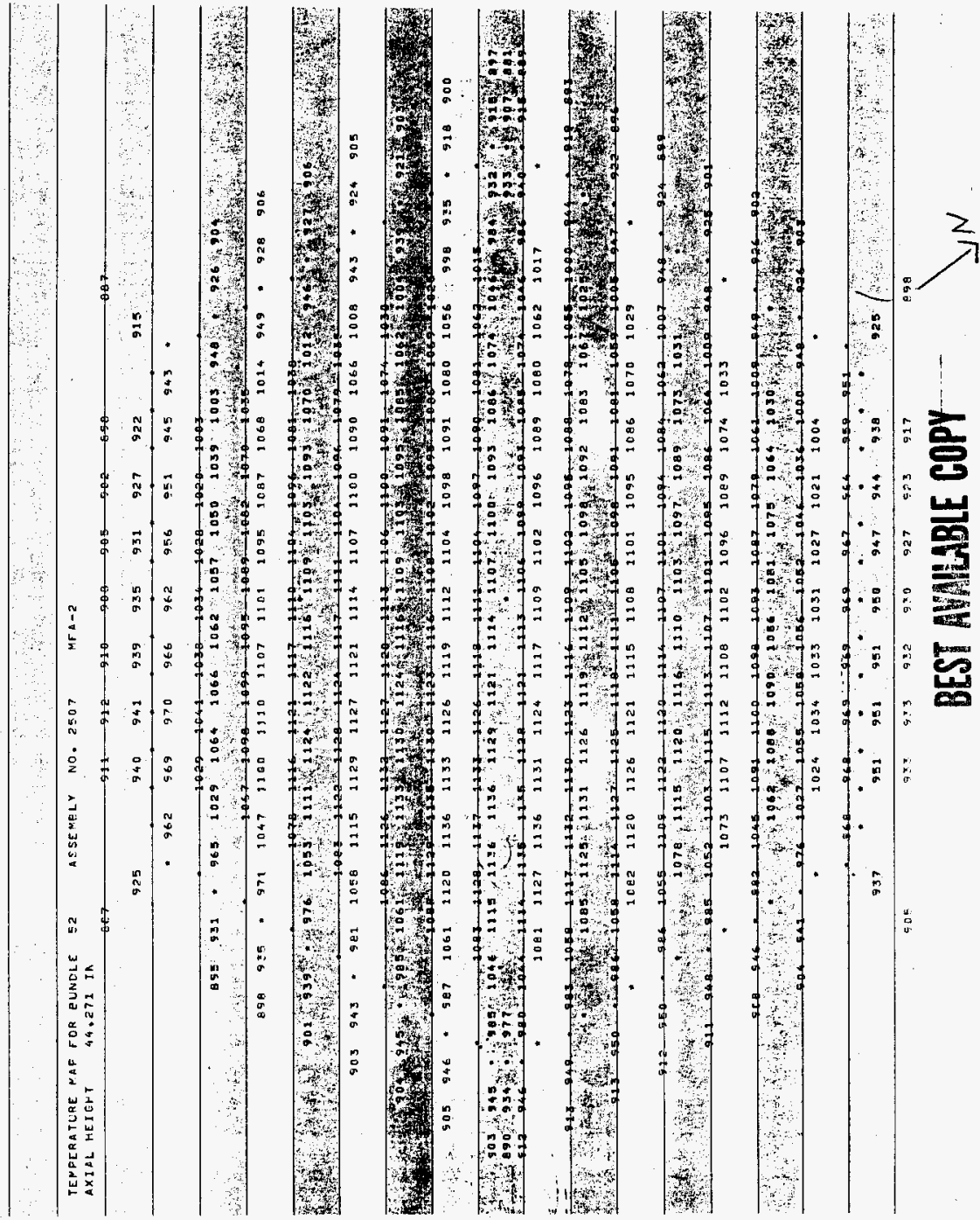


Table B1OB.13. MFA-1 Sodium Subchannel Temperatures at Elevation of Upper Axial Blanket for BOC 10B

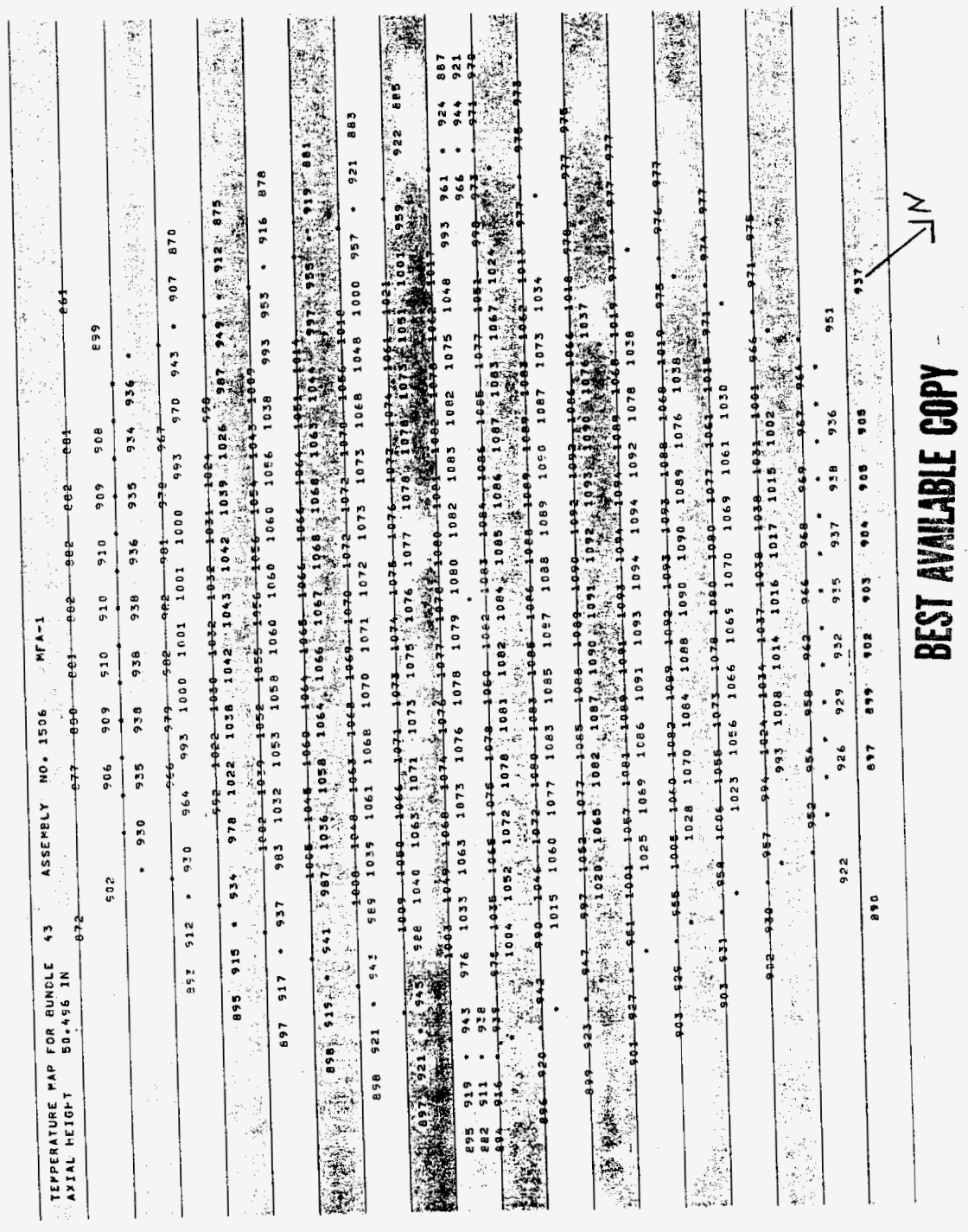


Table B10B.14. MFA-2 Sodium Subchannel Temperatures at Elevation of Upper Axial Blanket for BOC $10 B$

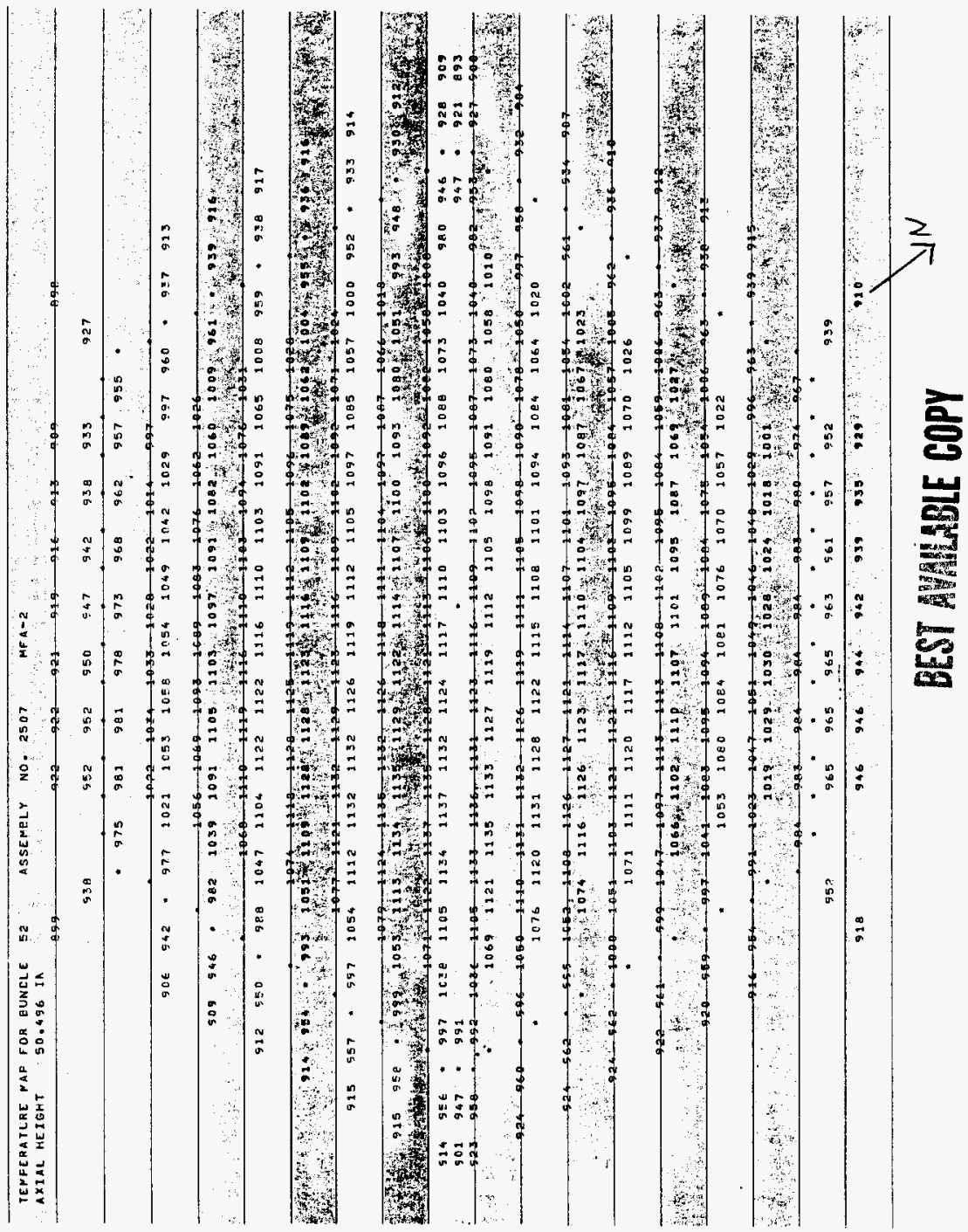




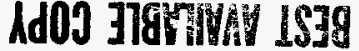

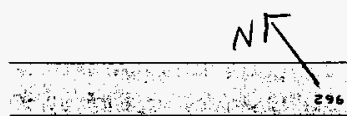

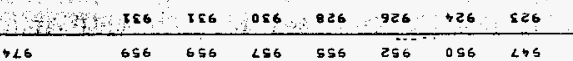

$\therefore$ its $\$ 46.656,656,456,556,256,096,045$. W 3 W

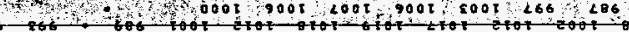

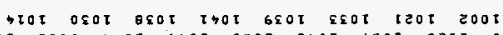

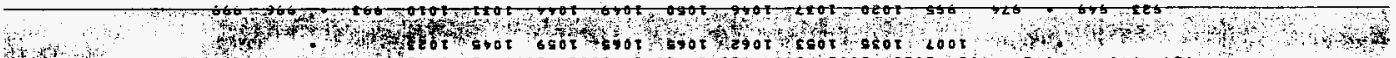
$\stackrel{\infty}{0}$

-

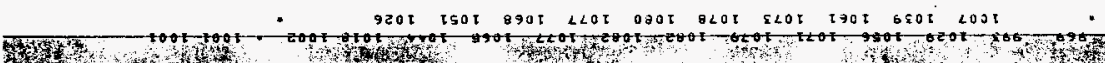

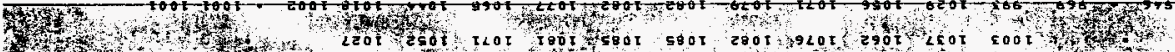

$$
\text { * }
$$

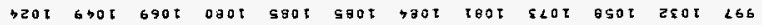
7.

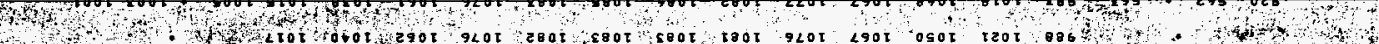

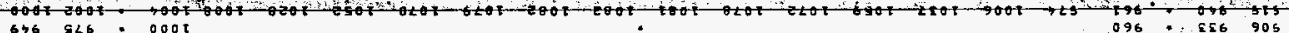

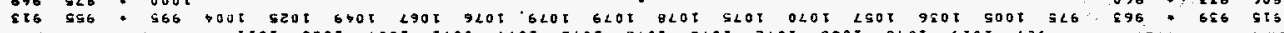

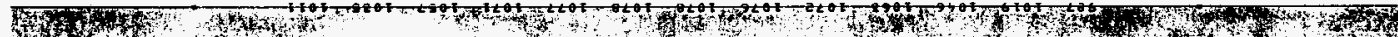

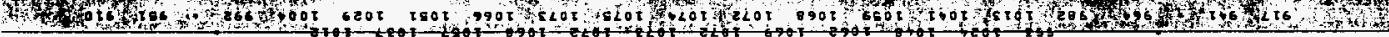

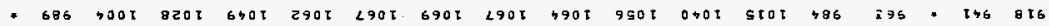

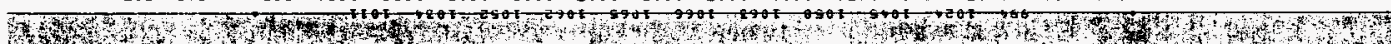

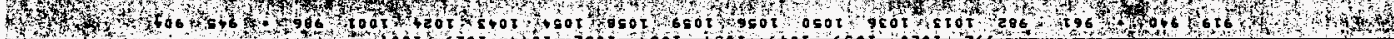

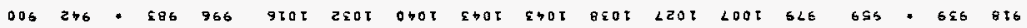

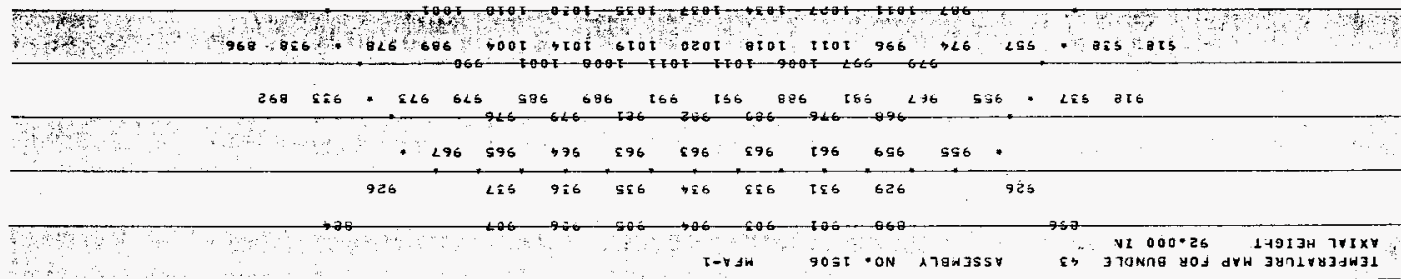


Table B10B.16. MFA-2 Sodium Subchannel Temperatures at Top of Fuel Pin Bundle for BOC 10B

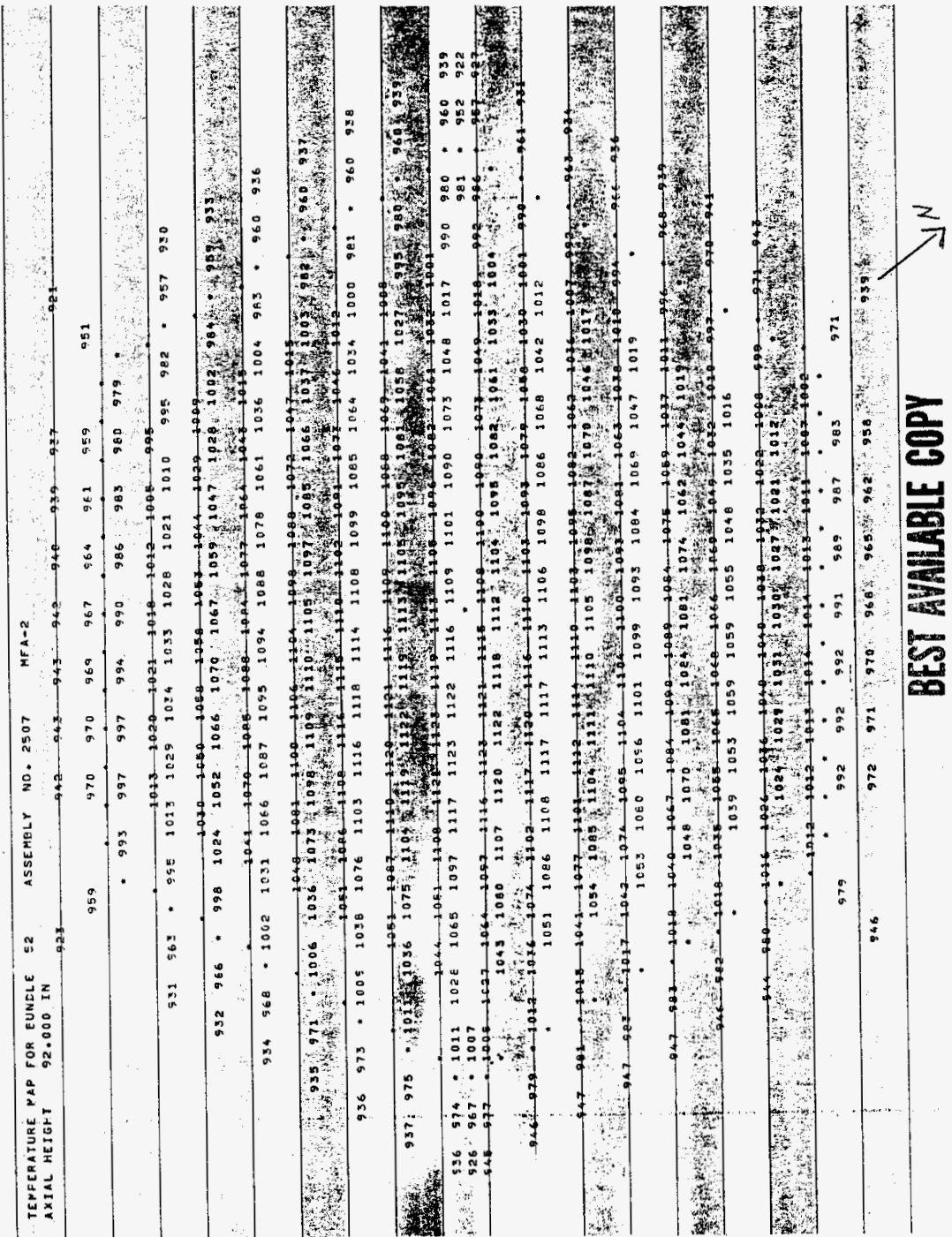


Table E10B.1. Fission Power Generated in MFA-1, MFA-2 and Neighboring Assemblies at EOC 10B

\begin{tabular}{lcccc} 
CORE & $* * * * * *$ & \multicolumn{2}{c}{ POWER IN MEGAWATTS } & $* * * * * *$ \\
POS & BELOW CORE & IN CORE & ABOVE CORE & TOTAL PWR \\
1506 & $4.147 \mathrm{E}-02$ & $3.600 \mathrm{E}+00$ & $3.064 \mathrm{E}-02$ & $3.672 \mathrm{E}+00$ \\
1404 & $9.507 \mathrm{E}-02$ & $4.277 \mathrm{E}+00$ & $6.009 \mathrm{E}-02$ & $4.432 \mathrm{E}+00$ \\
1405 & $0.000 \mathrm{E}+00$ & $6.248 \mathrm{E}+00$ & $0.000 \mathrm{E}+00$ & $6.248 \mathrm{E}+00$ \\
1505 & $7.608 \mathrm{E}-03$ & $4.130 \mathrm{E}+00$ & $6.111 \mathrm{E}-03$ & $4.143 \mathrm{E}+00$ \\
1507 & $1.043 \mathrm{E}-02$ & $3.040 \mathrm{E}+00$ & $8.763 \mathrm{E}-03$ & $3.060 \mathrm{E}+00$ \\
1606 & $7.433 \mathrm{E}-03$ & $3.351 \mathrm{E}+00$ & $6.214 \mathrm{E}-03$ & $3.365 \mathrm{E}+00$ \\
1607 & $9.673 \mathrm{E}-03$ & $2.711 \mathrm{E}+00$ & $8.021 \mathrm{E}-03$ & $2.729 \mathrm{E}+00$ \\
2507 & $4.353 \mathrm{E}-02$ & $3.746 \mathrm{E}+00$ & $3.353 \mathrm{E}-02$ & $3.823 \mathrm{E}+00$ \\
2405 & $1.103 \mathrm{E}-02$ & $3.904 \mathrm{E}+00$ & $9.356 \mathrm{E}-03$ & $3.925 \mathrm{E}+00$ \\
2506 & $7.814 \mathrm{E}-03$ & $4.175 \mathrm{E}+00$ & $6.790 \mathrm{E}-03$ & $4.190 \mathrm{E}+00$ \\
2508 & $1.258 \mathrm{E}-02$ & $4.150 \mathrm{E}+00$ & $1.046 \mathrm{E}-02$ & $4.173 \mathrm{E}+00$ \\
2607 & $8.646 \mathrm{E}-03$ & $3.251 \mathrm{E}+00$ & $7.244 \mathrm{E}-03$ & $3.267 \mathrm{E}+00$ \\
2608 & $6.043 \mathrm{E}-03$ & $3.165 \mathrm{E}+00$ & $5.465 \mathrm{E}-03$ & $3.176 \mathrm{E}+00$ \\
2609 & $6.872 \mathrm{E}-03$ & $3.982 \mathrm{E}+00$ & $6.211 \mathrm{E}-03$ & $3.995 \mathrm{E}+00$
\end{tabular}

Table E10B.2. Assembly Averaged Total Flux for MFA-1 and MFA-2 at EOC $10 B$

$\begin{array}{ccc}\text { Assembly } & \text { Core } & \text { Total Flux } \\ \text { PFA-1 } & 1506 & \left(\mathrm{n} / \mathrm{cm}^{2}-\mathrm{sec}\right) \\ \text { MFA-2 } & 2507 & 2.848 \mathrm{E}+15 \\ \text { MFA } & 2.948 \mathrm{E}+15\end{array}$


Table ElOB.3. Axial Distribution of Total Flux, Fast Flux and Power in MFA-1 at EOC $10 \mathrm{~B}$

$\begin{array}{rccc}\text { z }(\mathrm{cm}) & \text { Total Flux } & \text { Flux }>0.1 \mathrm{MeV} & \text { Power } \\ -97.78 & 2.6556 \mathrm{E}-02 & 1.3311 \mathrm{E}-02 & 0.0000 \mathrm{E}+00 \\ -92.78 & 5.7032 \mathrm{E}-02 & 2.5707 \mathrm{E}-02 & 0.0000 \mathrm{E}+00 \\ -87.78 & 9.1393 \mathrm{E}-02 & 4.1152 \mathrm{E}-02 & 0.0000 \mathrm{E}+00 \\ -82.78 & 1.3221 \mathrm{E}-01 & 6.1421 \mathrm{E}-02 & 0.0000 \mathrm{E}+00 \\ -77.78 & 1.8205 \mathrm{E}-01 & 8.8716 \mathrm{E}-02 & 0.0000 \mathrm{E}+00 \\ -72.15 & 2.3834 \mathrm{E}-01 & 1.2408 \mathrm{E}-01 & 0.0000 \mathrm{E}+00 \\ -65.90 & 2.9425 \mathrm{E}-01 & 1.6878 \mathrm{E}-01 & 0.0000 \mathrm{E}+00 \\ -60.35 & 3.5314 \mathrm{E}-01 & 2.2955 \mathrm{E}-01 & 4.8073 \mathrm{E}-02 \\ -55.49 & 4.3135 \mathrm{E}-01 & 3.1083 \mathrm{E}-01 & 5.8282 \mathrm{E}-02 \\ -50.62 & 5.2237 \mathrm{E}-01 & 4.1682 \mathrm{E}-01 & 7.3766 \mathrm{E}-02 \\ -47.17 & 5.9540 \mathrm{E}-01 & 5.1685 \mathrm{E}-01 & 9.1281 \mathrm{E}-02 \\ -43.58 & 6.7219 \mathrm{E}-01 & 6.3005 \mathrm{E}-01 & 7.1728 \mathrm{E}-01 \\ -38.45 & 7.8345 \mathrm{E}-01 & 7.6728 \mathrm{E}-01 & 8.0557 \mathrm{E}-01 \\ -33.32 & 8.8740 \mathrm{E}-01 & 8.8458 \mathrm{E}-01 & 9.0324 \mathrm{E}-01 \\ -28.20 & 9.7932 \mathrm{E}-01 & 9.8288 \mathrm{E}-01 & 9.7144 \mathrm{E}-01 \\ -23.07 & 1.0577 \mathrm{E}+00 & 1.0647 \mathrm{E}+00 & 1.0477 \mathrm{E}+00 \\ -17.94 & 1.1211 \mathrm{E}+00 & 1.1297 \mathrm{E}+00 & 1.1097 \mathrm{E}+00 \\ -12.82 & 1.1687 \mathrm{E}+00 & 1.1777 \mathrm{E}+00 & 1.1434 \mathrm{E}+00 \\ -7.69 & 1.2005 \mathrm{E}+00 & 1.2098 \mathrm{E}+00 & 1.1741 \mathrm{E}+00 \\ -2.56 & 1.2160 \mathrm{E}+00 & 1.2257 \mathrm{E}+00 & 1.1892 \mathrm{E}+00 \\ 2.56 & 1.2150 \mathrm{E}+00 & 1.2251 \mathrm{E}+00 & 1.1920 \mathrm{E}+00 \\ 7.69 & 1.1973 \mathrm{E}+00 & 1.2078 \mathrm{E}+00 & 1.1747 \mathrm{E}+00 \\ 12.82 & 1.1627 \mathrm{E}+00 & 1.1735 \mathrm{E}+00 & 1.1409 \mathrm{E}+00 \\ 17.94 & 1.1114 \mathrm{E}+00 & 1.1225 \mathrm{E}+00 & 1.1093 \mathrm{E}+00 \\ 23.07 & 1.0427 \mathrm{E}+00 & 1.0526 \mathrm{E}+00 & 1.0414 \mathrm{E}+00 \\ 28.20 & 9.5759 \mathrm{E}-01 & 9.6441 \mathrm{E}-01 & 9.5755 \mathrm{E}-01 \\ 33.32 & 8.5765 \mathrm{E}-01 & 8.5853 \mathrm{E}-01 & 8.8248 \mathrm{E}-01 \\ 38.45 & 7.4477 \mathrm{E}-01 & 7.3329 \mathrm{E}-01 & 7.7260 \mathrm{E}-01 \\ 43.58 & 6.2448 \mathrm{E}-01 & 5.8979 \mathrm{E}-01 & 6.6739 \mathrm{E}-01 \\ 47.17 & 5.4275 \mathrm{E}-01 & 4.7724 \mathrm{E}-01 & 7.4707 \mathrm{E}-02 \\ 50.62 & 4.6655 \mathrm{E}-01 & 3.7967 \mathrm{E}-01 & 5.7303 \mathrm{E}-02 \\ 55.49 & 3.7180 \mathrm{E}-01 & 2.7652 \mathrm{E}-01 & 4.1724 \mathrm{E}-02 \\ 60.35 & 2.8787 \mathrm{E}-01 & 1.9594 \mathrm{E}-01 & 3.0995 \mathrm{E}-02 \\ 64.98 & 2.3396 \mathrm{E}-01 & 1.4764 \mathrm{E}-01 & 0.0000 \mathrm{E}+00 \\ 69.39 & 2.0126 \mathrm{E}-01 & 1.2142 \mathrm{E}-01 & 0.0000 \mathrm{E}+00 \\ 76.46 & 1.5393 \mathrm{E}-01 & 8.6116 \mathrm{E}-02 & 0.0000 \mathrm{E}+00 \\ 86.80 & 1.0102 \mathrm{E}-01 & 5.1935 \mathrm{E}-02 & 0.0000 \mathrm{E}+00 \\ 98.14 & 5.8524 \mathrm{E}-02 & 2.8396 \mathrm{E}-02 & 0.0000 \mathrm{E}+00 \\ 110.00 & 2.5111 \mathrm{E}-02 & 1.2728 \mathrm{E}-02 & 0.0000 \mathrm{E}+00 \\ & & & \end{array}$


Table E10B.4. Axial Distribution of Total Flux, Fast Flux and Power in MFA-2 at EOC $10 B$

$\begin{array}{rccc}\text { Z }(\mathrm{cm}) & \text { Total Flux } & \text { Flux }>0.1 \mathrm{MeV} & \begin{array}{c}\text { Power } \\ -97.78\end{array} \\ -92.78 & 2.4343 \mathrm{E}-02 & 1.2478 \mathrm{E}-02 & 0.0000 \mathrm{E}+00 \\ -9.2264 \mathrm{E}-02 & 2.4073 \mathrm{E}-02 & 0.0000 \mathrm{E}+00 \\ -87.78 & 8.3781 \mathrm{E}-02 & 3.8498 \mathrm{E}-02 & 0.0000 \mathrm{E}+00 \\ -82.78 & 1.2141 \mathrm{E}-01 & 5.7431 \mathrm{E}-02 & 0.0000 \mathrm{E}+00 \\ -77.78 & 1.6797 \mathrm{E}-01 & 8.3004 \mathrm{E}-02 & 0.0000 \mathrm{E}+00 \\ -72.15 & 2.2192 \mathrm{E}-01 & 1.1645 \mathrm{E}-01 & 0.0000 \mathrm{E}+00 \\ -65.90 & 2.7786 \mathrm{E}-01 & 1.5972 \mathrm{E}-01 & 0.0000 \mathrm{E}+00 \\ -60.35 & 3.3789 \mathrm{E}-01 & 2.1907 \mathrm{E}-01 & 4.8702 \mathrm{E}-02 \\ -55.49 & 4.1625 \mathrm{E}-01 & 2.9863 \mathrm{E}-01 & 5.9101 \mathrm{E}-02 \\ -50.62 & 5.0746 \mathrm{E}-01 & 4.0350 \mathrm{E}-01 & 7.4249 \mathrm{E}-02 \\ -47.17 & 5.8144 \mathrm{E}-01 & 5.0374 \mathrm{E}-01 & 9.1260 \mathrm{E}-02 \\ -43.58 & 6.5967 \mathrm{E}-01 & 6.1810 \mathrm{E}-01 & 7.1220 \mathrm{E}-01 \\ -38.45 & 7.7387 \mathrm{E}-01 & 7.5769 \mathrm{E}-01 & 7.9880 \mathrm{E}-01 \\ -33.32 & 8.8079 \mathrm{E}-01 & 8.7784 \mathrm{E}-01 & 8.9793 \mathrm{E}-01 \\ -28.20 & 9.7550 \mathrm{E}-01 & 9.7917 \mathrm{E}-01 & 9.6699 \mathrm{E}-01 \\ -23.07 & 1.0566 \mathrm{E}+00 & 1.0639 \mathrm{E}+00 & 1.0456 \mathrm{E}+00 \\ -17.94 & 1.1226 \mathrm{E}+00 & 1.1316 \mathrm{E}+00 & 1.1101 \mathrm{E}+00 \\ -12.82 & 1.1723 \mathrm{E}+00 & 1.1817 \mathrm{E}+00 & 1.1448 \mathrm{E}+00 \\ -7.69 & 1.2055 \mathrm{E}+00 & 1.2151 \mathrm{E}+00 & 1.1770 \mathrm{E}+00 \\ -2.56 & 1.2216 \mathrm{E}+00 & 1.2316 \mathrm{E}+00 & 1.1926 \mathrm{E}+00 \\ 2.56 & 1.2204 \mathrm{E}+00 & 1.2307 \mathrm{E}+00 & 1.1948 \mathrm{E}+00 \\ 7.69 & 1.2018 \mathrm{E}+00 & 1.2125 \mathrm{E}+00 & 1.1766 \mathrm{E}+00 \\ 12.82 & 1.1661 \mathrm{E}+00 & 1.1769 \mathrm{E}+00 & 1.1419 \mathrm{E}+00 \\ 17.94 & 1.1136 \mathrm{E}+00 & 1.1243 \mathrm{E}+00 & 1.1097 \mathrm{E}+00 \\ 23.07 & 1.0439 \mathrm{E}+00 & 1.0532 \mathrm{E}+00 & 1.0409 \mathrm{E}+00 \\ 28.20 & 9.5798 \mathrm{E}-01 & 9.6416 \mathrm{E}-01 & 9.5666 \mathrm{E}-01 \\ 33.32 & 8.5773 \mathrm{E}-01 & 8.5803 \mathrm{E}-01 & 8.8274 \mathrm{E}-01 \\ 38.45 & 7.4497 \mathrm{E}-01 & 7.3319 \mathrm{E}-01 & 7.7482 \mathrm{E}-01 \\ 43.58 & 6.2503 \mathrm{E}-01 & 5.9026 \mathrm{E}-01 & 6.7578 \mathrm{E}-01 \\ 47.17 & 5.4250 \mathrm{E}-01 & 4.7582 \mathrm{E}-01 & 7.7442 \mathrm{E}-02 \\ 50.62 & 4.6424 \mathrm{E}-01 & 3.7539 \mathrm{E}-01 & 5.9987 \mathrm{E}-02 \\ 55.49 & 3.6663 \mathrm{E}-01 & 2.6980 \mathrm{E}-01 & 4.4168 \mathrm{E}-02 \\ 60.35 & 2.8101 \mathrm{E}-01 & 1.8885 \mathrm{E}-01 & 3.3072 \mathrm{E}-02 \\ 64.98 & 2.2784 \mathrm{E}-01 & 1.4187 \mathrm{E}-01 & 0.0000 \mathrm{E}+00 \\ 69.39 & 1.9574 \mathrm{E}-01 & 1.1675 \mathrm{E}-01 & 0.0000 \mathrm{E}+00 \\ 76.46 & 1.4946 \mathrm{E}-01 & 8.2926 \mathrm{E}-02 & 0.0000 \mathrm{E}+00 \\ 86.80 & 9.7887 \mathrm{E}-02 & 4.9962 \mathrm{E}-02 & 0.0000 \mathrm{E}+00 \\ 98.14 & 5.6532 \mathrm{E}-02 & 2.7207 \mathrm{E}-02 & 0.0000 \mathrm{E}+00 \\ 110.00 & 2.3989 \mathrm{E}-02 & 1.2054 \mathrm{E}-02 & 0.0000 \mathrm{E}+00\end{array}$




\section{WHC-SD-FF-ANAL-009 REV 0}

Table E1OB.5. Fission Power Distribution by Pin in MFA-1 at EOC 10B

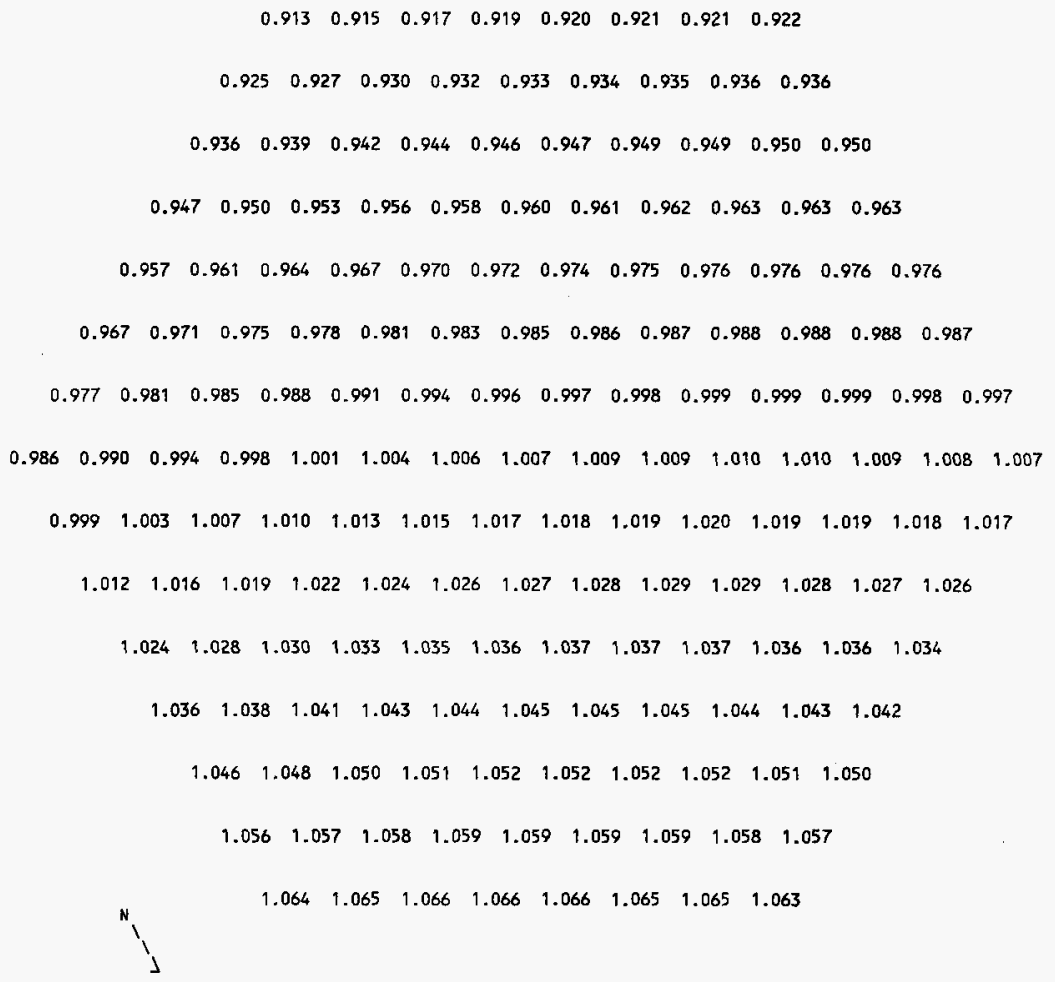


Table E10B.6. Fission Power Distribution by Pin in MFA-2 at EOC 10B

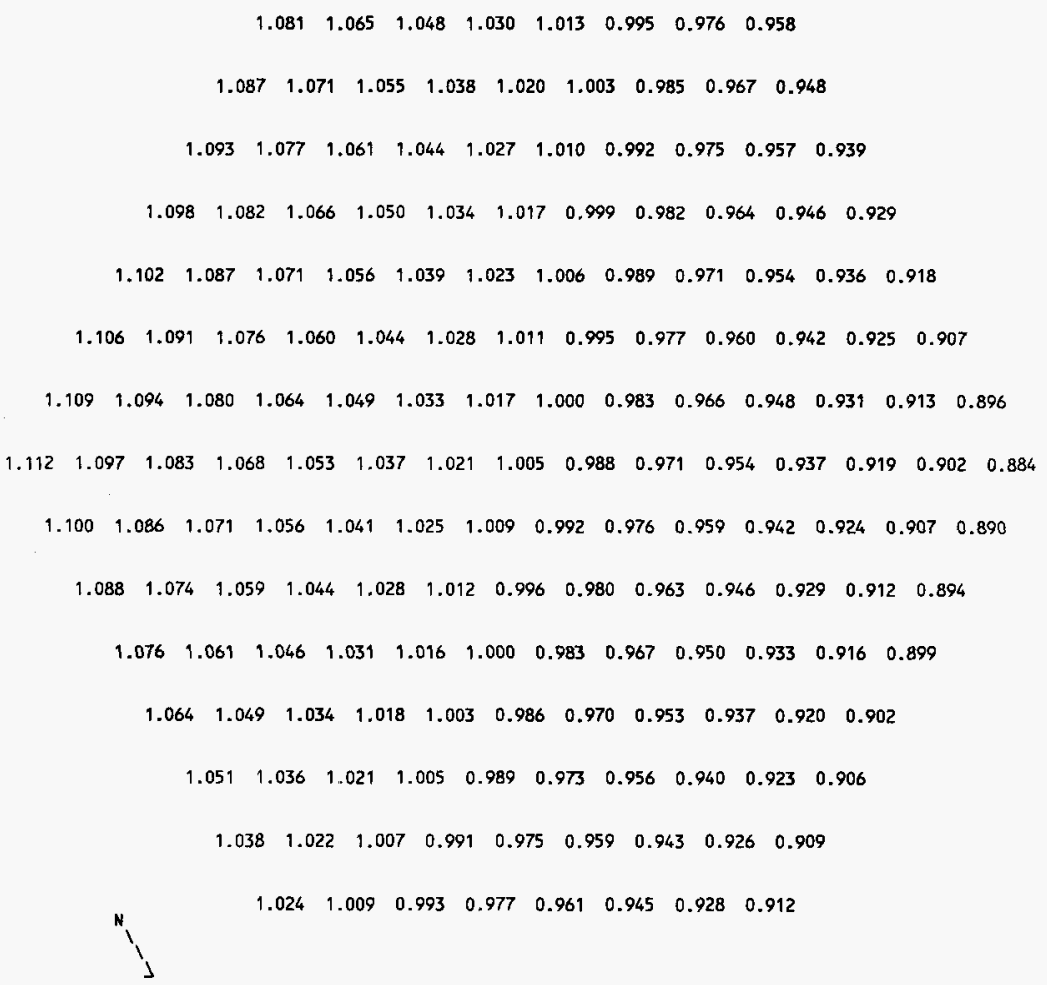


Table E10B.7. Total Flux Distribution by Pin in MFA-1 at EOC 10B

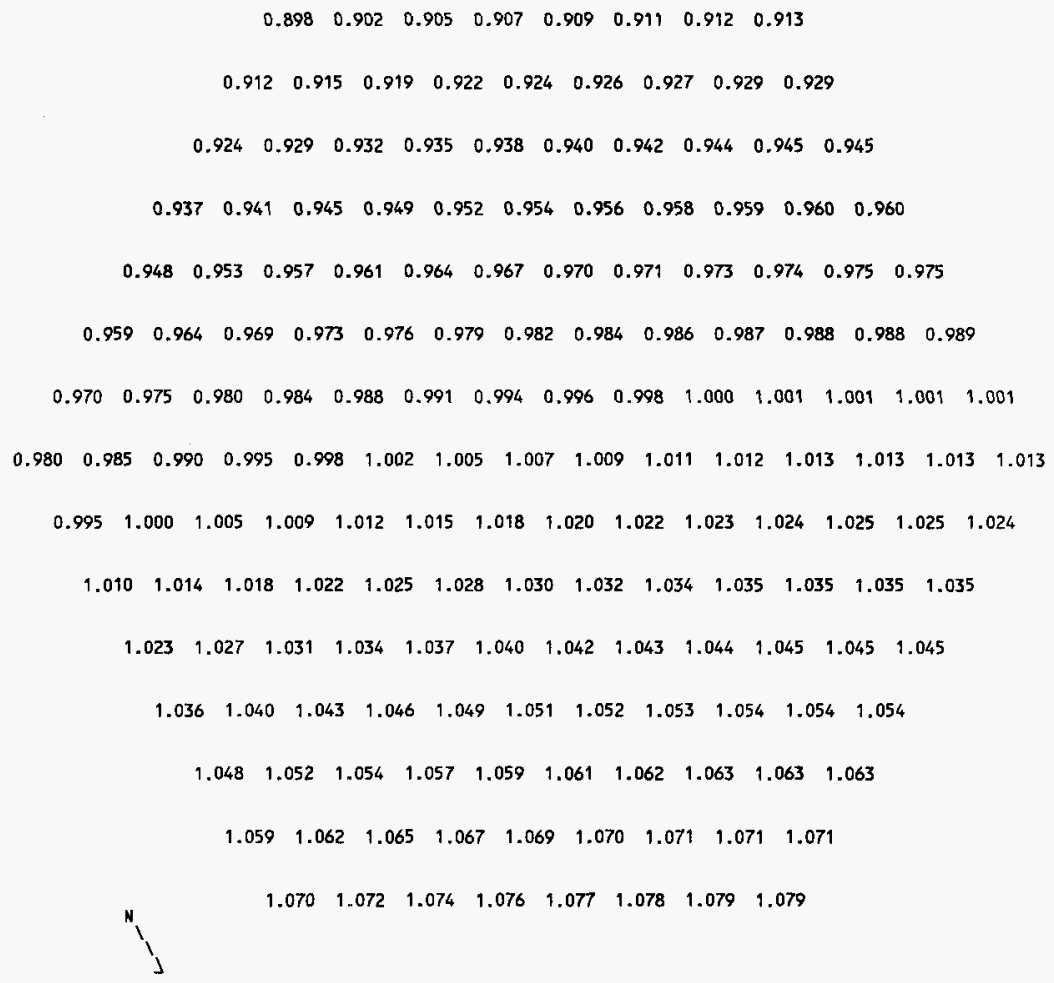


Table E10B.8. Total Flux Distribution by Pin in MFA-2 at EOC 10B

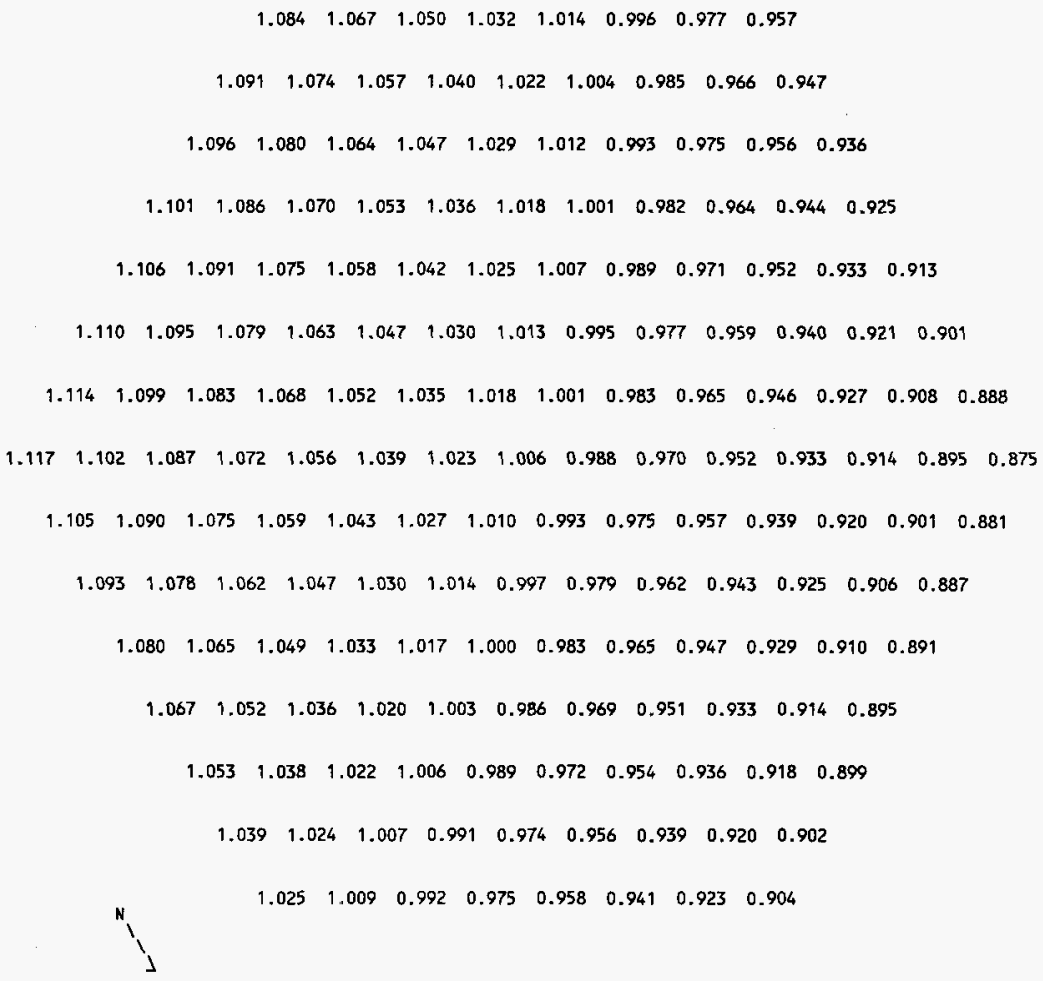


Table E10B.9. MFA-1 and MFA-2 Duct Wall Flux Greater Than $0.1 \mathrm{MeV}$ at EOC $10 \mathrm{~B}$

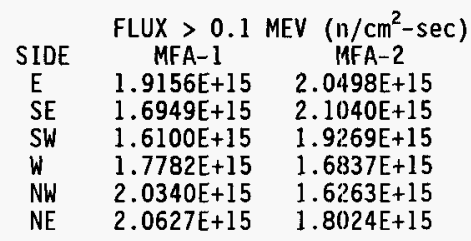

Table ElOB.10. Assembly outlet Temperatures and Flow Rates at EOC $10 \mathrm{~B}$

$\begin{array}{lccc}\text { CORE } & \text { OUTLET TEMP. } & \text { (DEG F) } & \text { FLOW RATE } \\ \text { P0S. } & \text { MEASURED } & \text { CALCULATED } & (\text { LB } / H \text { ) } \\ 1506 & 1016 & 1019 & 115440 \\ 1404 & 903 & 897 & 228160 \\ 1405 & 1022 & 1023 & 188170 \\ 1505 & 902 & 893 & 209040 \\ 1507 & 861 & 842 & 209040 \\ 1606 & 894 & 892 & 171430 \\ 1607 & 866 & 855 & 171430 \\ 2507 & 1024 & 1027 & 115440 \\ 2405 & 889 & 881 & 209060 \\ 2506 & 904 & 8912 & 209040 \\ 2508 & 909 & 899 & 209040 \\ 2607 & 901 & 88.9 & 164410 \\ 2608 & 886 & 883 & 171430 \\ 2609 & 937 & 943 & 164410\end{array}$




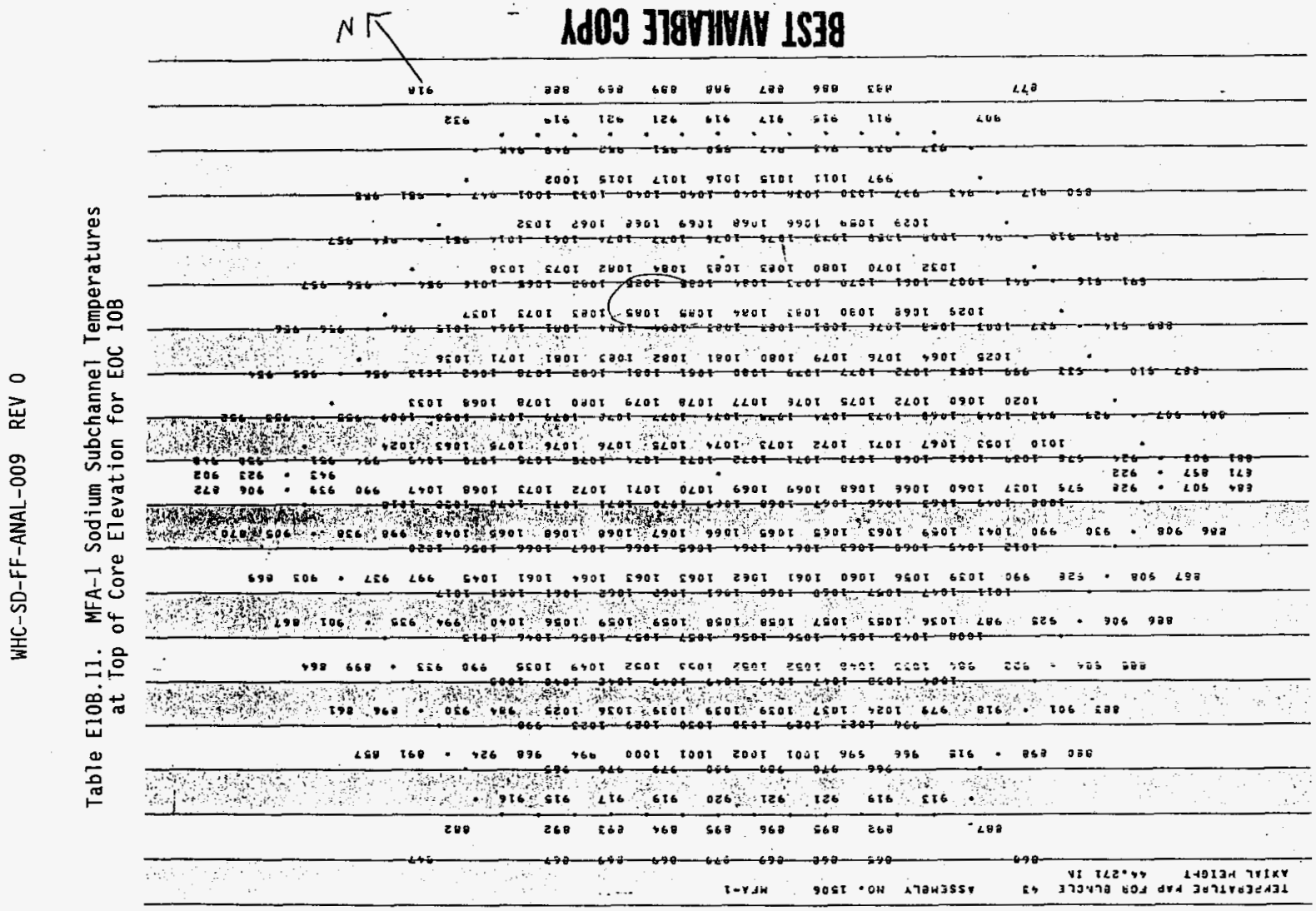




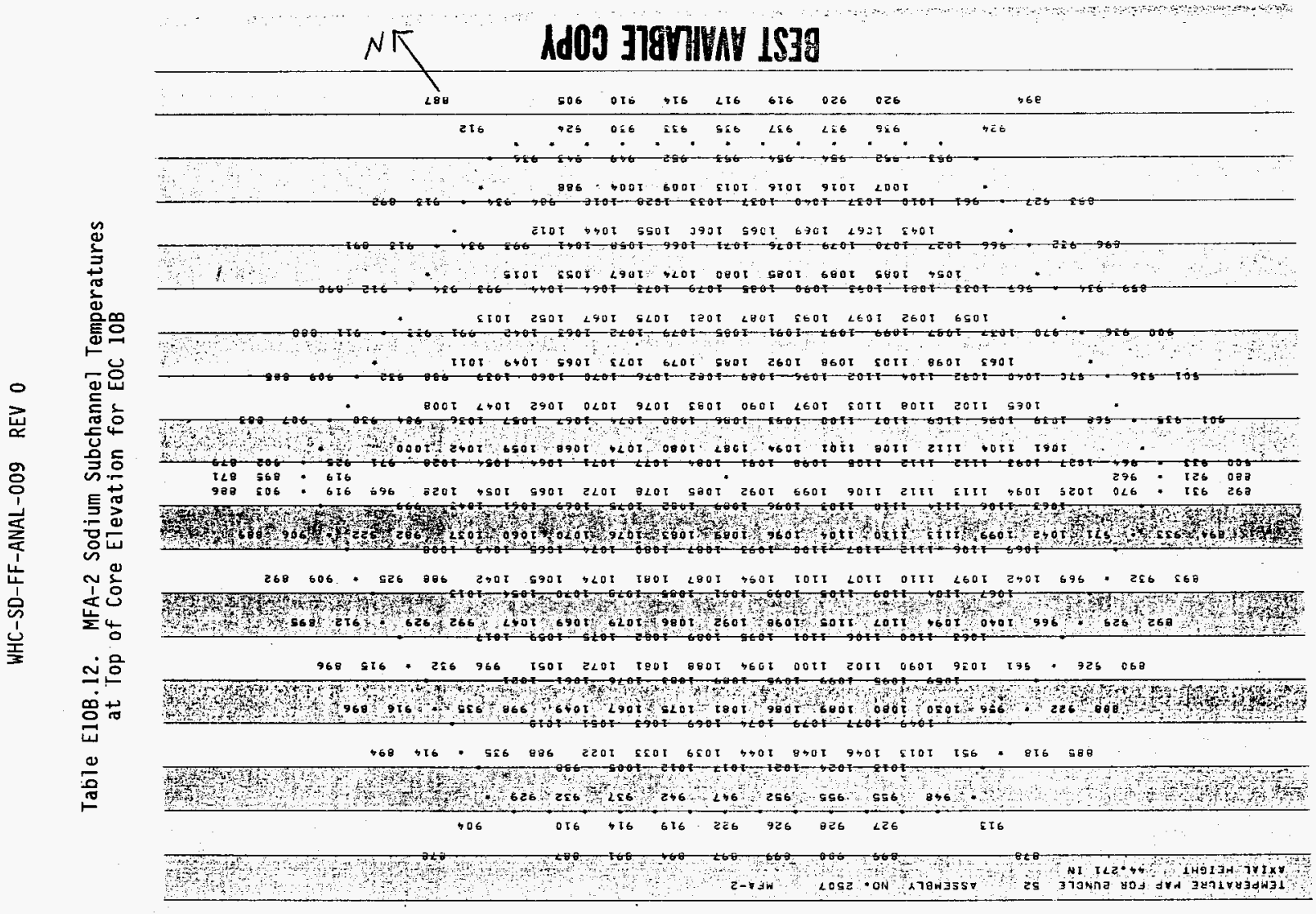




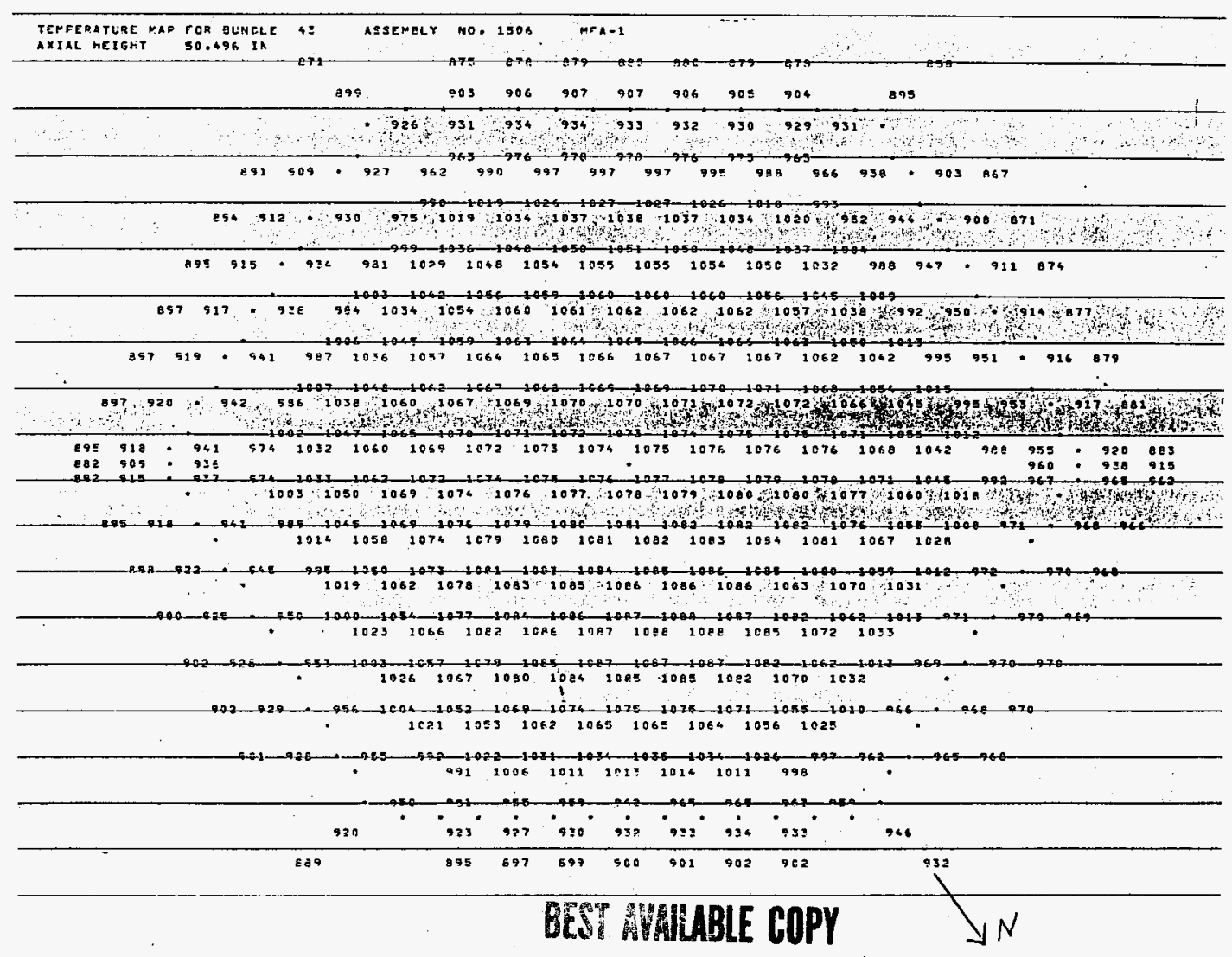

$\stackrel{\vec{\sigma}}{\frac{\sigma}{\sigma}}$

밈

용

$\stackrel{ \pm}{ \pm}$

옥.

옥주 죽

돈 
Table E10B.14. MFA-2 Sodium Subchannel Temperatures at Elevation of Upper Axial Blanket for EOC $10 B$

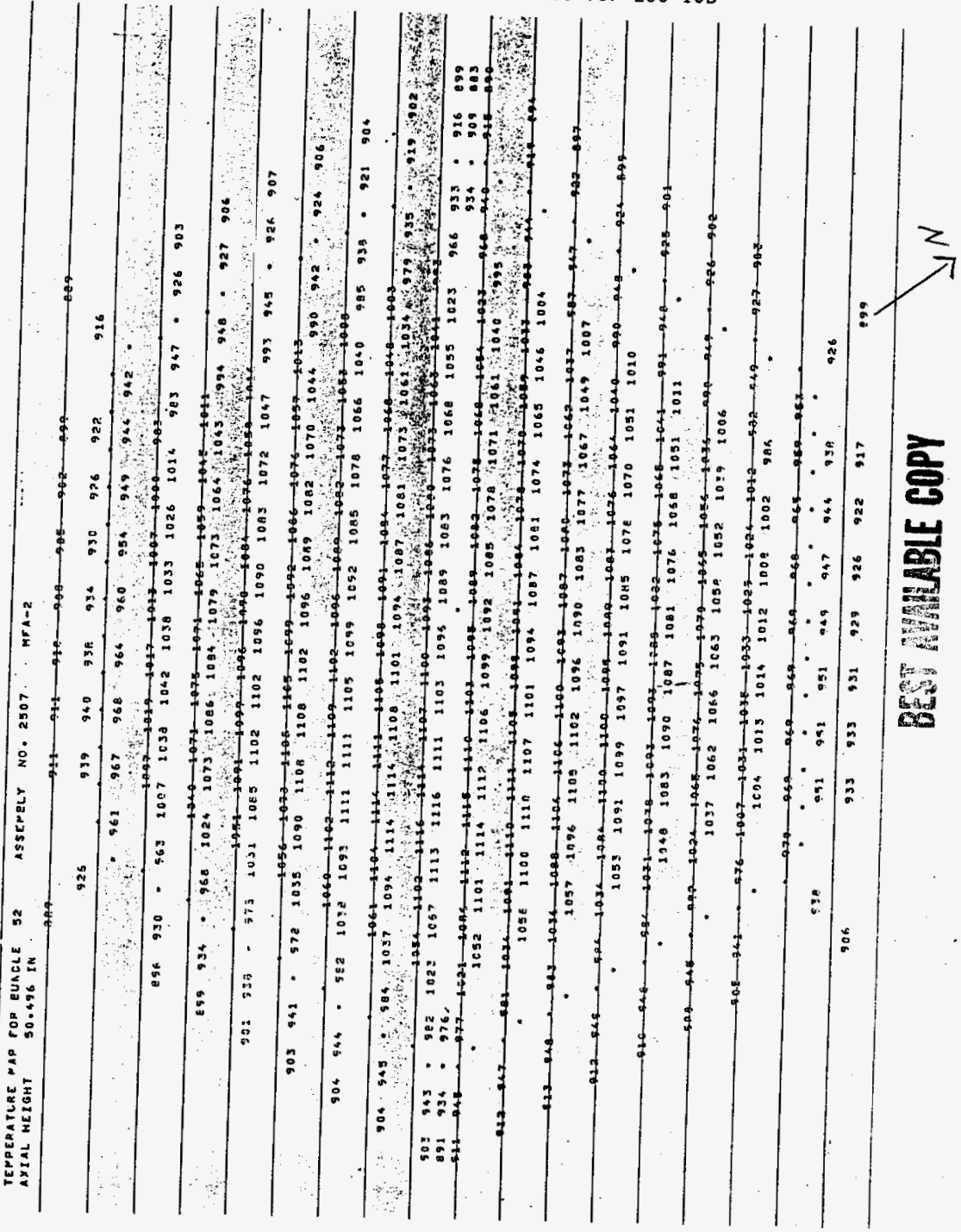


Table E10B.15. MFA-1 Sodium Subchannel Temperatures at Top of Fuel Pin Bundle for EOC $10 B$

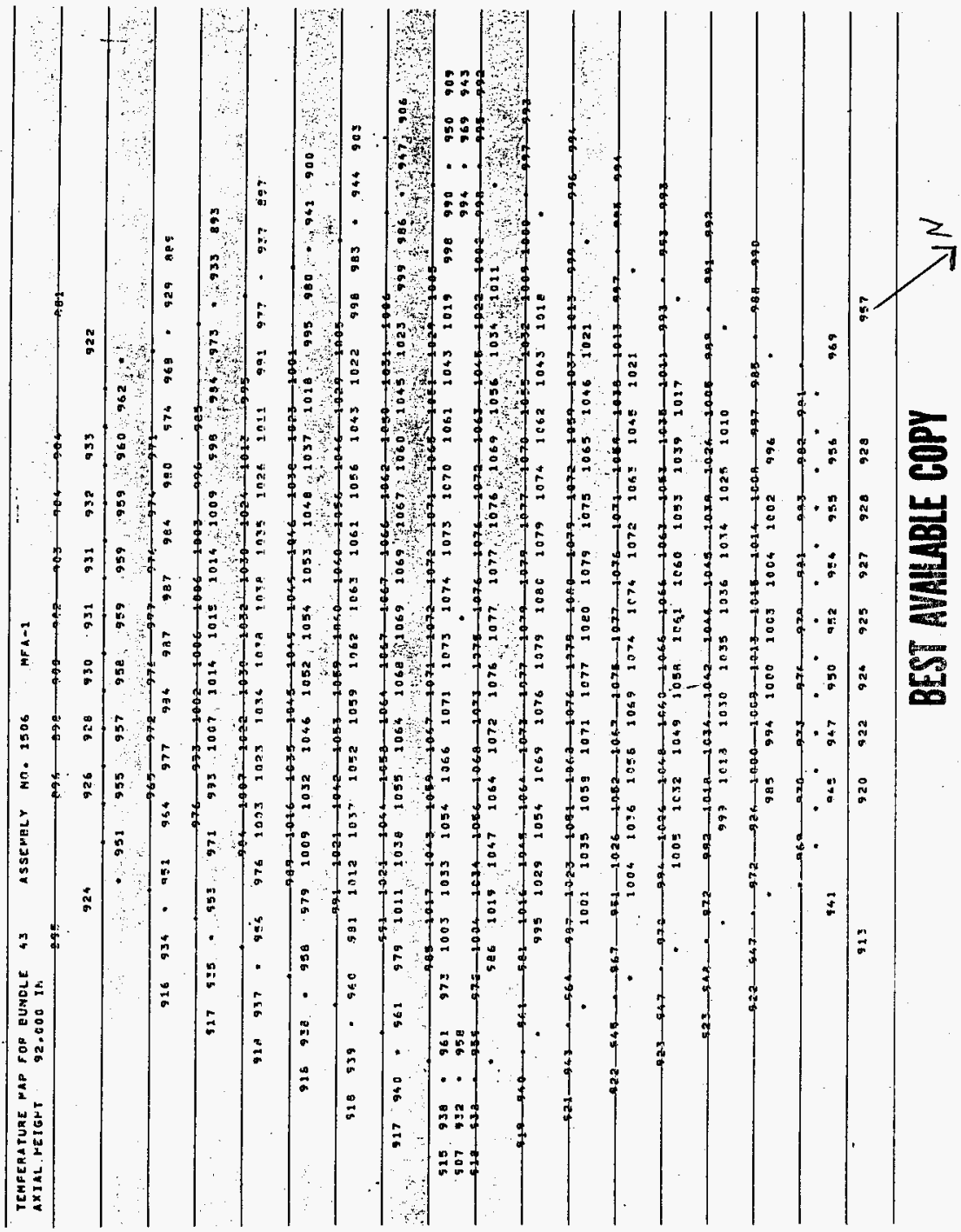


Table ElOB.16. MFA-2 Sodium Subchannel Temperatures at Top of Fuel Pin Bundle for EOC $10 B$

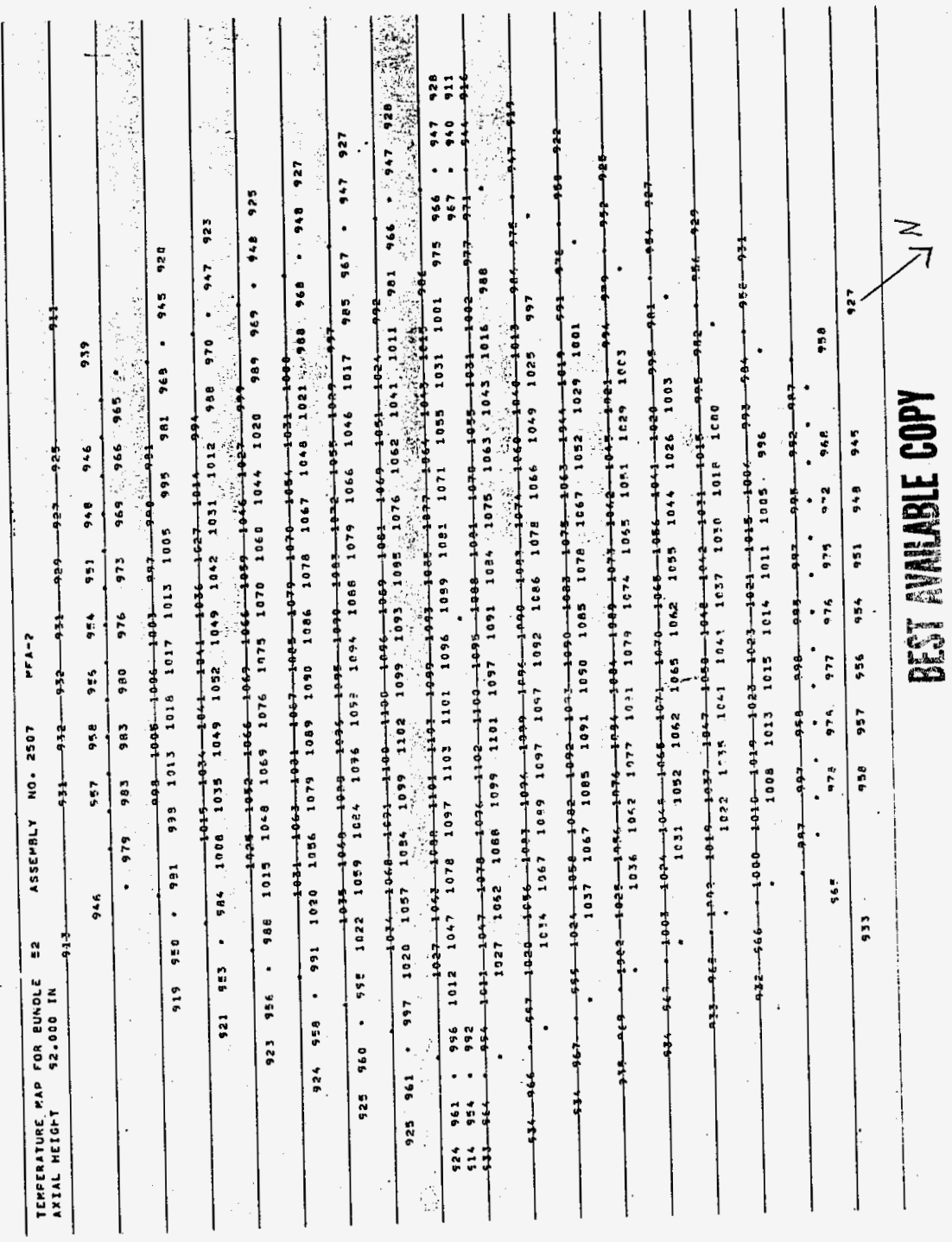


Table E10B.17. Composition of MFA-1 at EOC 10B

\begin{tabular}{|c|c|c|c|c|c|}
\hline & Axial & $(\mathrm{cm})$ & & Atom Densi & $-\mathrm{cm})$ \\
\hline $\begin{array}{c}\text { vel } \\
1\end{array}$ & $\begin{array}{r}\text { Lower } \\
-100.28\end{array}$ & $\begin{array}{r}\text { Upper } \\
-75.28\end{array}$ & Constituent & Fresh & \\
\hline & & & $\begin{array}{l}\mathrm{Na}-23 \\
\text { SS-316 }\end{array}$ & $\begin{array}{l}5.3150 \mathrm{E}-03 \\
6.4090 \mathrm{E}-02\end{array}$ & $\begin{array}{l}5.3150 \mathrm{E}-03 \\
6.4090 \mathrm{E}-02\end{array}$ \\
\hline 2 & -75.28 & -62.78 & & & \\
\hline & & & $\begin{array}{l}\mathrm{Na}-23 \\
\text { SS-316 }\end{array}$ & $\begin{array}{l}1.3177 \mathrm{E}-02 \\
3.4175 \mathrm{E}-02\end{array}$ & $\begin{array}{l}1.3177 \mathrm{E}-02 \\
3.4175 \mathrm{E}-02\end{array}$ \\
\hline 3 & -62.78 & -46.14 & $\begin{array}{l}\text { U-235 } \\
U-238 \\
\text { Pu-239 } \\
\text { Pu-240 } \\
\text { FP } \\
\text { O-16 } \\
\mathrm{Na}-23 \\
\text { SS-316 }\end{array}$ & $\begin{array}{l}1.4143 \mathrm{E}-05 \\
7.0573 \mathrm{E}-03 \\
0.0000 \mathrm{E}+00 \\
0.0000 \mathrm{E}+00 \\
0.0000 \mathrm{E}+00 \\
1.4143 \mathrm{E}-02 \\
9.7416 \mathrm{E}-03 \\
1.9073 \mathrm{E}-02\end{array}$ & $\begin{array}{l}1.2238 \mathrm{E}-05 \\
6.9566 \mathrm{E}-03 \\
8.8941 \mathrm{E}-05 \\
1.7886 \mathrm{E}-06 \\
1.1291 \mathrm{E}-05 \\
1.4143 \mathrm{E}-02 \\
9.7416 \mathrm{E}-03 \\
1.9073 \mathrm{E}-02\end{array}$ \\
\hline 4 & -46.14 & -30.76 & $\begin{array}{l}U-235 \\
U-238 \\
P u-239 \\
P u-240 \\
P u-241 \\
P u-242 \\
\text { Am-241 } \\
\text { FP } \\
0-16 \\
\text { Na-23 } \\
\text { SS-316 }\end{array}$ & $\begin{array}{l}1.0840 \mathrm{E}-05 \\
4.4499 \mathrm{E}-03 \\
1.7520 \mathrm{E}-03 \\
2.2340 \mathrm{E}-04 \\
1.9280 \mathrm{E}-05 \\
4.7430 \mathrm{E}-06 \\
7.6680 \mathrm{E}-07 \\
0.0000 \mathrm{E}+00 \\
1.2670 \mathrm{E}-02 \\
9.8100 \mathrm{E}-03 \\
1.8990 \mathrm{E}-02\end{array}$ & $\begin{array}{l}9.5078 \mathrm{E}-06 \\
4.3731 \mathrm{E}-03 \\
1.6001 \mathrm{E}-03 \\
2.5209 \mathrm{E}-04 \\
2.1258 \mathrm{E}-05 \\
4.9086 \mathrm{E}-06 \\
1.4957 \mathrm{E}-06 \\
1.9784 \mathrm{E}-04 \\
1.2670 \mathrm{E}-02 \\
9.8100 \mathrm{E}-03 \\
1.8990 \mathrm{E}-02\end{array}$ \\
\hline 5 & -30.76 & -15.38 & $\begin{array}{l}U-235 \\
U-238 \\
P u-239 \\
P u-240 \\
P u-241 \\
P u-242 \\
\text { Am-241 } \\
F P \\
O-16 \\
\mathrm{Na}-23 \\
\text { SS }-316\end{array}$ & $\begin{array}{l}1.0840 \mathrm{E}-05 \\
4.4499 \mathrm{E}-03 \\
1.7520 \mathrm{E}-03 \\
2.2340 \mathrm{E}-04 \\
1.9280 \mathrm{E}-05 \\
4.7430 \mathrm{E}-06 \\
7.6680 \mathrm{E}-07 \\
0.0000 \mathrm{E}+00 \\
1.2670 \mathrm{E}-02 \\
9.8100 \mathrm{E}-03 \\
1.8990 \mathrm{E}-02\end{array}$ & $\begin{array}{l}9.2007 \mathrm{E}-06 \\
4.3525 \mathrm{E}-03 \\
1.5596 \mathrm{E}-03 \\
2.5435 \mathrm{E}-04 \\
2.1405 \mathrm{E}-05 \\
4.9098 \mathrm{E}-06 \\
1.4732 \mathrm{E}-06 \\
2.5693 \mathrm{E}-04 \\
1.2670 \mathrm{E}-02 \\
9.8100 \mathrm{E}-03 \\
1.8990 \mathrm{E}-02\end{array}$ \\
\hline 6 & -15.38 & 0.00 & $\begin{array}{l}\text { U-235 } \\
\text { U-238 } \\
\mathrm{Pu}-239 \\
\mathrm{Pu}-240 \\
\mathrm{Pu}-241 \\
\mathrm{Pu}-242 \\
\mathrm{Am}-241 \\
\mathrm{FP} \\
\mathrm{O}-16 \\
\mathrm{Na}-23 \\
\mathrm{SS}-316\end{array}$ & $\begin{array}{l}1.0840 \mathrm{E}-05 \\
4.4499 \mathrm{E}-03 \\
1.7520 \mathrm{E}-03 \\
2.2340 \mathrm{E}-04 \\
1.9280 \mathrm{E}-05 \\
4.7430 \mathrm{E}-06 \\
7.6680 \mathrm{E}-07 \\
0.0000 \mathrm{E}+00 \\
1.2670 \mathrm{E}-02 \\
9.8100 \mathrm{E}-03 \\
1.8990 \mathrm{E}-02\end{array}$ & $\begin{array}{l}9.0293 \mathrm{E}-06 \\
4.3414 \mathrm{E}-03 \\
1.5388 \mathrm{E}-03 \\
2.5720 \mathrm{E}-04 \\
2.1727 \mathrm{E}-05 \\
4.9304 \mathrm{E}-06 \\
1.4646 \mathrm{E}-06 \\
2.8557 \mathrm{E}-04 \\
1.2670 \mathrm{E}-02 \\
9.8100 \mathrm{E}-03 \\
1.8990 \mathrm{E}-02\end{array}$ \\
\hline
\end{tabular}


$\begin{array}{lll}7 & 0.00 & 15.38\end{array}$

$\begin{array}{lll}8 & 15.38 & 30.76\end{array}$

$\begin{array}{lll}\mathrm{U}-235 & 1.0840 \mathrm{E}-05 & 9.0781 \mathrm{E}-06 \\ \mathrm{U}-238 & 4.4499 \mathrm{E}-03 & 4.3446 \mathrm{E}-03 \\ \mathrm{Pu}-239 & 1.7520 \mathrm{E}-03 & 1.5446 \mathrm{E}-03 \\ \mathrm{Pu}-240 & 2.2340 \mathrm{E}-04 & 2.5624 \mathrm{E}-04 \\ \mathrm{Pu}-241 & 1.9280 \mathrm{E}-05 & 2.1618 \mathrm{E}-05 \\ \mathrm{Pu}-242 & 4.7430 \mathrm{E}-06 & 4.9231 \mathrm{E}-06 \\ \mathrm{Am}-241 & 7.6680 \mathrm{E}-07 & 1.4670 \mathrm{E}-06 \\ \mathrm{FP} & 0.0000 \mathrm{E}+00 & 2.7762 \mathrm{E}-04 \\ \mathrm{O}-16 & 1.2670 \mathrm{E}-02 & 1.2670 \mathrm{E}-02 \\ \mathrm{Na}-23 & 9.8100 \mathrm{E}-03 & 9.8100 \mathrm{E}-03 \\ \mathrm{SS}-316 & 1.8990 \mathrm{E}-02 & 1.8990 \mathrm{E}-02\end{array}$

$\mathrm{U}-235 \quad 1.0840 \mathrm{E}-05$

9.3414E-06

$U-238$

4. 4499E-03

$\mathrm{Pu}-239 \quad 1.7520 \mathrm{E}-03$

4. 3613E-03

$\mathrm{Pu}-240$

2. $2340 \mathrm{E}-04$

$\mathrm{Pu}-241$

$\mathrm{Pu}-242$

$1.9280 \mathrm{E}-05$

Am-241

4. $7430 \mathrm{E}-06$

FP

$7.6680 \mathrm{E}-07$

$1.5758 \mathrm{E}-03$

2. $5158 \mathrm{E}-04$

2.1101E-05

4. $8900 \mathrm{E}-06$

1. $4800 \mathrm{E}-06$

$0-16$

$0.0000 \mathrm{E}+00$

2.3484E-04

$\mathrm{Na}-23$

1.2670E-02

1. $2670 \mathrm{E}-02$

SS -316

$9.8100 \mathrm{E}-03$

9.8100E-03

$\begin{array}{lll}9 & 30.76 & 46.14\end{array}$

$\begin{array}{lll}\mathrm{U}-235 & 1.0840 \mathrm{E}-05 & 9.7206 \mathrm{E}-06 \\ \mathrm{U}-238 & 4.4499 \mathrm{E}-03 & 4.3858 \mathrm{E}-03 \\ \mathrm{Pu}-239 & 1.7520 \mathrm{E}-03 & 1.6238 \mathrm{E}-03 \\ \mathrm{Pu}-240 & 2.2340 \mathrm{E}-04 & 2.4697 \mathrm{E}-04 \\ \mathrm{Pu}-241 & 1.9280 \mathrm{E}-05 & 2.0681 \mathrm{E}-05 \\ \mathrm{Pu}-242 & 4.7430 \mathrm{E}-06 & 4.8705 \mathrm{E}-06 \\ \mathrm{Am}-241 & 7.6680 \mathrm{E}-07 & 1.5038 \mathrm{E}-06 \\ \mathrm{FP} & 0.0000 \mathrm{E}+00 & 1.6702 \mathrm{E}-04 \\ 0-16 & 1.2670 \mathrm{E}-02 & 1.2670 \mathrm{E}-02 \\ \mathrm{Na}-23 & 9.8100 \mathrm{E}-03 & 9.8100 \mathrm{E}-03 \\ \mathrm{SS}-316 & 1.8990 \mathrm{E}-02 & 1.8990 \mathrm{E}-02\end{array}$

$\begin{array}{lll}10 & 46.14 & 62.78\end{array}$

$\begin{array}{lll}\mathrm{U}-235 & 1.4143 \mathrm{E}-05 & 1.2827 \mathrm{E}-05 \\ \mathrm{U}-238 & 7.0573 \mathrm{E}-03 & 6.9864 \mathrm{E}-03 \\ \mathrm{Pu}-239 & 0.0000 \mathrm{E}+00 & 6.3300 \mathrm{E}-05 \\ \mathrm{Pu}-240 & 0.0000 \mathrm{E}+00 & 8.2206 \mathrm{E}-07 \\ \mathrm{FP} & 0.0000 \mathrm{E}+00 & 7.6817 \mathrm{E}-06 \\ 0-16 & 1.4143 \mathrm{E}-02 & 1.4143 \mathrm{E}-02 \\ \mathrm{Na}-23 & 9.7416 \mathrm{E}-03 & 9.7416 \mathrm{E}-03 \\ \mathrm{SS}-316 & 1.9073 \mathrm{E}-02 & 1.9073 \mathrm{E}-02\end{array}$

$11 \quad 62.78 \quad 116.00$

$\begin{array}{lll}\mathrm{Na}-23 & 8.2400 \mathrm{E}-03 & 8.2400 \mathrm{E}-03 \\ \mathrm{SS}-316 & 2.5562 \mathrm{E}-02 & 2.5562 \mathrm{E}-02\end{array}$


Table E1OB.18. Composition of MFA-2 at EOC 10B

\begin{tabular}{|c|c|c|c|c|c|}
\hline xial & Axial $F$ & ge $(\mathrm{cm})$ & & Atom Dens & $(a / b-c m)$ \\
\hline $\begin{array}{c}\text { Level } \\
1\end{array}$ & $\begin{array}{r}\text { Lower } \\
-100.28\end{array}$ & $\begin{array}{r}\text { Upper } \\
-75.28\end{array}$ & Constituent & Fresh & nd of Cycle \\
\hline & & & $\begin{array}{l}\mathrm{Na}-23 \\
\mathrm{SS}-316\end{array}$ & $\begin{array}{l}5.3150 \mathrm{E}-03 \\
6.4090 \mathrm{E}-02\end{array}$ & $\begin{array}{l}5.3150 \mathrm{E}-03 \\
6.4090 \mathrm{E}-02\end{array}$ \\
\hline 2 & -75.28 & -62.78 & & & \\
\hline & & & $\begin{array}{l}\mathrm{Na}-23 \\
\mathrm{SS}-316\end{array}$ & $\begin{array}{l}1.3177 \mathrm{E}-02 \\
3.4175 \mathrm{E}-02\end{array}$ & $\begin{array}{l}1.3177 \mathrm{E}-02 \\
3.4175 \mathrm{E}-02\end{array}$ \\
\hline 3 & -62.78 & -46.14 & & & \\
\hline & & & $\begin{array}{l}U-235 \\
U-238 \\
\mathrm{Pu}-239 \\
\mathrm{Pu}-240 \\
\mathrm{FP} \\
\mathrm{O}-16 \\
\mathrm{Na}-23 \\
\mathrm{SS}-316\end{array}$ & $\begin{array}{l}1.4143 \mathrm{E}-05 \\
7.0573 \mathrm{E}-03 \\
0.0000 \mathrm{E}+00 \\
0.0000 \mathrm{E}+00 \\
0.0000 \mathrm{E}+00 \\
1.4143 \mathrm{E}-02 \\
9.7416 \mathrm{E}-03 \\
1.9073 \mathrm{E}-02\end{array}$ & $\begin{array}{l}1.2168 \mathrm{E}-05 \\
6.9539 \mathrm{E}-03 \\
9.1185 \mathrm{E}-05 \\
1.9680 \mathrm{E}-06 \\
1.1653 \mathrm{E}-05 \\
1.4143 \mathrm{E}-02 \\
9.7416 \mathrm{E}-03 \\
1.9073 \mathrm{E}-02\end{array}$ \\
\hline 4 & -46.14 & -30.76 & & & \\
\hline & & & $\begin{array}{l}U-235 \\
U-238 \\
P u-239 \\
P u-240 \\
P u-241 \\
P u-242 \\
\text { Am-241 } \\
\text { FP } \\
0-16 \\
\mathrm{Na}-23 \\
\text { SS }-316\end{array}$ & $\begin{array}{l}1.0160 \mathrm{E}-05 \\
4.4270 \mathrm{E}-03 \\
1.7483 \mathrm{E}-03 \\
2.2290 \mathrm{E}-04 \\
1.9040 \mathrm{E}-05 \\
4.7330 \mathrm{E}-06 \\
7.5700 \mathrm{E}-07 \\
0.0000 \mathrm{E}+00 \\
1.2760 \mathrm{E}-02 \\
9.8100 \mathrm{E}-03 \\
1.8990 \mathrm{E}-02\end{array}$ & $\begin{array}{l}8.8595 \mathrm{E}-06 \\
4.3471 \mathrm{E}-03 \\
1.5916 \mathrm{E}-03 \\
2.5360 \mathrm{E}-04 \\
2.1302 \mathrm{E}-05 \\
4.9107 \mathrm{E}-06 \\
1.4735 \mathrm{E}-06 \\
2.0350 \mathrm{E}-04 \\
1.2760 \mathrm{E}-02 \\
9.8100 \mathrm{E}-03 \\
1.8990 \mathrm{E}-02\end{array}$ \\
\hline 5 & -30.76 & -15.38 & & & \\
\hline & & & $\begin{array}{l}\mathrm{U}-235 \\
\mathrm{U}-238 \\
\mathrm{Pu}-239 \\
\mathrm{Pu}-240 \\
\mathrm{Pu}-241 \\
\mathrm{Pu}-242 \\
\mathrm{Am}-241 \\
\mathrm{FP} \\
\mathrm{O}-16 \\
\mathrm{Na}-23 \\
\mathrm{SS}-316\end{array}$ & $\begin{array}{l}1.0160 \mathrm{E}-05 \\
4.4270 \mathrm{E}-03 \\
1.7483 \mathrm{E}-03 \\
2.2290 \mathrm{E}-04 \\
1.9040 \mathrm{E}-05 \\
4.7330 \mathrm{E}-06 \\
7.5700 \mathrm{E}-07 \\
0.0000 \mathrm{E}+00 \\
1.2760 \mathrm{E}-02 \\
9.8100 \mathrm{E}-03 \\
1.8990 \mathrm{E}-02\end{array}$ & $\begin{array}{l}8.5513 \mathrm{E}-06 \\
4.3247 \mathrm{E}-03 \\
1.5486 \mathrm{E}-03 \\
2.5621 \mathrm{E}-04 \\
2.1487 \mathrm{E}-05 \\
4.9136 \mathrm{E}-06 \\
1.4501 \mathrm{E}-06 \\
2.6625 \mathrm{E}-04 \\
1.2760 \mathrm{E}-02 \\
9.8100 \mathrm{E}-03 \\
1.8990 \mathrm{E}-02\end{array}$ \\
\hline 6 & -15.38 & 0.00 & & & \\
\hline & & & $\begin{array}{l}\mathrm{U}-235 \\
\mathrm{U}-238 \\
\mathrm{Pu}-239 \\
\mathrm{Pu}-240 \\
\mathrm{Pu}-241 \\
\mathrm{Pu}-242 \\
\mathrm{Am}-241 \\
\mathrm{FP} \\
\mathrm{O}-16 \\
\mathrm{Na}-23 \\
\mathrm{SS}-316\end{array}$ & $\begin{array}{l}1.0160 \mathrm{E}-05 \\
4.4270 \mathrm{E}-03 \\
1.7483 \mathrm{E}-03 \\
2.2290 \mathrm{E}-04 \\
1.9040 \mathrm{E}-05 \\
4.7330 \mathrm{E}-06 \\
7.5700 \mathrm{E}-07 \\
0.0000 \mathrm{E}+00 \\
1.2760 \mathrm{E}-02 \\
9.8100 \mathrm{E}-03 \\
1.8990 \mathrm{E}-02\end{array}$ & $\begin{array}{l}8.3739 \mathrm{E}-06 \\
4.3124 \mathrm{E}-03 \\
1.5261 \mathrm{E}-03 \\
2.5941 \mathrm{E}-04 \\
2.1865 \mathrm{E}-05 \\
4.9377 \mathrm{E}-06 \\
1.4404 \mathrm{E}-06 \\
2.9760 \mathrm{E}-04 \\
1.2760 \mathrm{E}-02 \\
9.8100 \mathrm{E}-03 \\
1.8990 \mathrm{E}-02\end{array}$ \\
\hline
\end{tabular}




\begin{tabular}{|c|c|c|c|c|c|}
\hline \multirow[t]{2}{*}{7} & 0.00 & 15.38 & & & \\
\hline & & & $\begin{array}{l}U-235 \\
U-238 \\
P u-239 \\
P u-240 \\
P u-241 \\
P u-242 \\
\mathrm{Am}-241 \\
\mathrm{FP} \\
0-16 \\
\mathrm{Na}-23 \\
\mathrm{SS}-316\end{array}$ & $\begin{array}{l}1.0160 \mathrm{E}-05 \\
4.4270 \mathrm{E}-03 \\
1.7483 \mathrm{E}-03 \\
2.2290 \mathrm{E}-04 \\
1.9040 \mathrm{E}-05 \\
4.7330 \mathrm{E}-06 \\
7.5700 \mathrm{E}-07 \\
0.0000 \mathrm{E}+00 \\
1.2760 \mathrm{E}-02 \\
9.8100 \mathrm{E}-03 \\
1.8990 \mathrm{E}-02\end{array}$ & $\begin{array}{l}8.4177 \mathrm{E}-06 \\
4.3155 \mathrm{E}-03 \\
1.5314 \mathrm{E}-03 \\
2.5850 \mathrm{E}-04 \\
2.1756 \mathrm{E}-05 \\
4.9304 \mathrm{E}-06 \\
1.4426 \mathrm{E}-06 \\
2.9017 \mathrm{E}-04 \\
1.2760 \mathrm{E}-02 \\
9.8100 \mathrm{E}-03 \\
1.8990 \mathrm{E}-02\end{array}$ \\
\hline \multirow[t]{2}{*}{8} & 15.38 & 30.76 & & & \\
\hline & & & $\begin{array}{l}U-235 \\
U-238 \\
P u-239 \\
P u-240 \\
P u-241 \\
P u-242 \\
A m-241 \\
F P \\
O-16 \\
N a-23 \\
S S-316\end{array}$ & $\begin{array}{l}1.0160 \mathrm{E}-05 \\
4.4270 \mathrm{E}-03 \\
1.7483 \mathrm{E}-03 \\
2.2290 \mathrm{E}-04 \\
1.9040 \mathrm{E}-05 \\
4.7330 \mathrm{E}-06 \\
7.5700 \mathrm{E}-07 \\
0.0000 \mathrm{E}+00 \\
1.2760 \mathrm{E}-02 \\
9.8100 \mathrm{E}-03 \\
1.8990 \mathrm{E}-02\end{array}$ & $\begin{array}{l}8.6688 \mathrm{E}-06 \\
4.3327 \mathrm{E}-03 \\
1.5631 \mathrm{E}-03 \\
2.5373 \mathrm{E}-04 \\
2.1205 \mathrm{E}-05 \\
4.8955 \mathrm{E}-06 \\
1.4559 \mathrm{E}-06 \\
2.4655 \mathrm{E}-04 \\
1.2760 \mathrm{E}-02 \\
9.8100 \mathrm{E}-03 \\
1.8990 \mathrm{E}-02\end{array}$ \\
\hline \multirow[t]{2}{*}{9} & 30.76 & 46.14 & & & \\
\hline & & & $\begin{array}{l}U-235 \\
U-238 \\
P u-239 \\
P u-240 \\
P u-241 \\
P u-242 \\
A m-241 \\
F P \\
O-16 \\
N a-23 \\
S S-316\end{array}$ & $\begin{array}{l}1.0160 \mathrm{E}-05 \\
4.4270 \mathrm{E}-03 \\
1.7483 \mathrm{E}-03 \\
2.2290 \mathrm{E}-04 \\
1.9040 \mathrm{E}-05 \\
4.7330 \mathrm{E}-06 \\
7.5700 \mathrm{E}-07 \\
0.0000 \mathrm{E}+00 \\
1.2760 \mathrm{E}-02 \\
9.8100 \mathrm{E}-03 \\
1.8990 \mathrm{E}-02\end{array}$ & $\begin{array}{l}9.0368 \mathrm{E}-06 \\
4.3583 \mathrm{E}-03 \\
1.6125 \mathrm{E}-03 \\
2.4899 \mathrm{E}-04 \\
2.0764 \mathrm{E}-05 \\
4.8756 \mathrm{E}-06 \\
1.4806 \mathrm{E}-06 \\
1.7654 \mathrm{E}-04 \\
1.2760 \mathrm{E}-02 \\
9.8100 \mathrm{E}-03 \\
1.8990 \mathrm{E}-02\end{array}$ \\
\hline \multirow[t]{2}{*}{10} & 46.14 & 62.78 & & & \\
\hline & & & $\begin{array}{l}U-235 \\
U-238 \\
P u-239 \\
P u-240 \\
F P \\
0-16 \\
\mathrm{Na}-23 \\
\text { SS-316 }\end{array}$ & $\begin{array}{l}1.4143 \mathrm{E}-05 \\
7.0573 \mathrm{E}-03 \\
0.0000 \mathrm{E}+00 \\
0.0000 \mathrm{E}+00 \\
0.0000 \mathrm{E}+00 \\
1.4143 \mathrm{E}-02 \\
9.7416 \mathrm{E}-03 \\
1.9073 \mathrm{E}-02\end{array}$ & $\begin{array}{l}1.2691 \mathrm{E}-05 \\
6.9803 \mathrm{E}-03 \\
6.8652 \mathrm{E}-05 \\
1.0361 \mathrm{E}-06 \\
8.3704 \mathrm{E}-06 \\
1.4143 \mathrm{E}-02 \\
9.7416 \mathrm{E}-03 \\
1.9073 \mathrm{E}-02\end{array}$ \\
\hline \multirow[t]{2}{*}{11} & 62.78 & 116.00 & & & \\
\hline & & & $\begin{array}{l}\mathrm{Na}-23 \\
\mathrm{SS}-316\end{array}$ & $\begin{array}{l}8.2400 \mathrm{E}-03 \\
2.5562 \mathrm{E}-02\end{array}$ & $\begin{array}{l}8.2400 \mathrm{E}-03 \\
2.5562 \mathrm{E}-02\end{array}$ \\
\hline
\end{tabular}




\section{CHECKLIST FOR INDEPENDENT TECHNICAL REVIEN}

DOCUMENT REVIEWED

NUMBER:

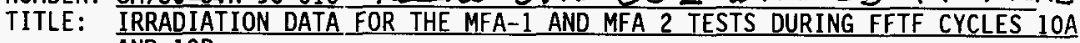
AND 10B

Reviewer(s):_R. F. Richard

I. Method(s) of Review

$(\checkmark)$ Input data clecked for accuracy

(W/A) Independent calculation performed

(w/A) Hand calculation

(W/A) Alternate computer code:

(wiA) Comparison to experiment or previous results

w/A ) Alternate method (define)

II. Checklist (either check or enter NA if not applied)

$(\checkmark)$ Task completely defined

(d) Activity consistent with task specification

$\omega / A)$ Necessary assumptions explicitly stated and supported

$(\checkmark)$ Resources properly identified and referenced

$(\checkmark)$ Resource documentation appropriate for this application

$(\sim)$ Input data explicitly stated

$(\checkmark)$ Input data verified to be consistent with original source

$(W(A)$ Geometric model adequate representation of actual geometry

(W/A) Material properties appropriate and reasonable

$(W / A)$ Mathematical derivations checked including dimensional consistency

(w/ H) Hand calculations checked for errors

(v) Assumptions explicitly stated and justified

(NA) Computer software appropriate for task and used within range of validity

$(W / A)$ Use of resource outside range of established validity is justified

(KA) Software runstreams correct and consistent with results

$W(A)$ Software outrut consistent with input

(w/A) Results consistent with applicable previous experimental or analytical findings

w/A Results and conclusions address all points and are consistent with task requirements and/or established limits or criteria

$(W / A)$ Conclusions consistent with analytical results and established limits

(WA) Uncertainty assessment appropriate and reasonable

() Other (define)

III. Comments: Data repental is comsistant unit riginal esta

IV. REVIEWER: R.F. luchand DATE: $10-4-96$

$$
142
$$




\begin{tabular}{|c|c|c|c|c|c|}
\hline \multicolumn{6}{|c|}{ DISTRIBUTION SHEET } \\
\hline To & \multirow{2}{*}{\multicolumn{3}{|c|}{$\begin{array}{l}\text { From } \\
\text { Criticality and Shielding }\end{array}$}} & \multicolumn{2}{|l|}{ Page 1 of 1} \\
\hline Distribution & & & & \multicolumn{2}{|c|}{$\begin{array}{l}\text { Date October 14, } \\
1996\end{array}$} \\
\hline \multicolumn{4}{|l|}{ Project Title/Work Order } & \multicolumn{2}{|c|}{ EDT No. 619215} \\
\hline \multicolumn{4}{|c|}{$\begin{array}{l}\text { Irradiation Data for the MFA-1 and MFA-2 Tests During FFTF } \\
\text { CYCLES } 10 \mathrm{~A} \text { and } 10 \mathrm{~B}\end{array}$} & \multicolumn{2}{|l|}{ ECN No. } \\
\hline Name & MSIN & $\begin{array}{c}\text { Text } \\
\text { With All } \\
\text { Attach. }\end{array}$ & Text Only & $\begin{array}{l}\text { Attach./ } \\
\text { Appendix } \\
\text { Only }\end{array}$ & $\begin{array}{l}\text { EDT/ECN } \\
\text { Only }\end{array}$ \\
\hline Central Files ( $1+$ Original) & A3-88 & $x$ & & & \\
\hline K. D. Dobbin & $\mathrm{HO}-35$ & $x$ & & & \\
\hline J. Greenborg & $\mathrm{H} 0-35$ & $x$ & & & \\
\hline J. R. Joiner & E6-28 & $x$ & & & \\
\hline D. S. Leach & A3-34 & $X$ & & & \\
\hline J. V. Nelson & $\mathrm{HO}-35$ & $x$ & & & \\
\hline R. J. Puigh & $\mathrm{HO}-31$ & $X$ & & & \\
\hline R. F. Richard & $\mathrm{HO}-35$ & $x$ & & & \\
\hline
\end{tabular}

